\title{
Topological Automorphic Forms
}

\author{
Mark Behrens \\ Tyler Lawson
}

\author{
Author address: \\ Department of Mathematics, Massachusetts Institute of Technology, \\ CAmbridge, MA 02140 \\ E-mail address: mbehrens@math.mit.edu \\ Department of Mathematics, University of Minnesota, Minneapolis, \\ MN 55455 \\ E-mail address: tlawson@math.umn.edu
}




\section{Contents}

Introduction $\quad$ ix

0.1. Background and motivation ix

0.2. Subject matter of this book xvii

0.3. Organization of this book xxiii

0.4. Acknowledgments xxv

Chapter 1. $p$-divisible groups 1

1.1. Definitions 1

1.2. Classification 3

Chapter 2. The Honda-Tate classification 5

2.1. Abelian varieties over finite fields 5

2.2. Abelian varieties over $\overline{\mathbb{F}}_{p} \quad 6$

$\begin{array}{ll}\text { Chapter 3. Tate modules and level structures } & 13\end{array}$

3.1. Tate modules of abelian varieties $\quad 13$

3.2. Virtual subgroups and quasi-isogenies 14

$\begin{array}{ll}3.3 . & \text { Level structures } \\ & 15\end{array}$

3.4. The Tate representation 16

3.5. Homomorphisms of abelian schemes 16

$\begin{array}{ll}\text { Chapter 4. Polarizations } & 19\end{array}$

$\begin{array}{ll}\text { 4.1. Polarizations } & 19\end{array}$

4.2. The Rosati involution $\quad 22$

4.3. The Weil pairing 22

4.4. Polarizations of $B$-linear abelian varieties 23

4.5. Induced polarizations 23

4.6. Classification of weak polarizations 24

$\begin{array}{lll}\text { Chapter 5. Forms and involutions } & 27\end{array}$

5.1. Hermitian forms 27

5.2. Unitary and similitude groups $\quad 30$

5.3. Classification of forms 31

Chapter 6. Shimura varieties of type $U(1, n-1) \quad 35$

6.1. Motivation 35

6.2. Initial data 36

6.3. Statement of the moduli problem 37

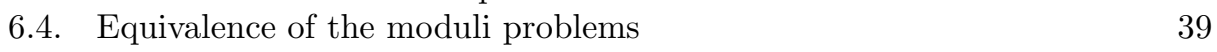

6.5. Moduli problems with level structure $\quad 41$

6.6. Shimura stacks 42 
Chapter 7. Deformation theory 45

7.1. Deformations of $p$-divisible groups 45

7.2. Serre-Tate theory 47

7.3. Deformation theory of points of $S h \quad 47$

Chapter 8. Topological automorphic forms 51

8.1. The generalized Hopkins-Miller theorem 51

8.2. The descent spectral sequence 54

8.3. Application to Shimura stacks $\quad 56$

Chapter 9. Relationship to automorphic forms $\quad 57$

9.1. Alternate description of $\operatorname{Sh}\left(K^{p}\right) \quad 57$

9.2. Description of $\operatorname{Sh}\left(K^{p}\right)_{F} \quad 59$

9.3. Description of $\operatorname{Sh}\left(K^{p}\right)_{\mathbb{C}} \quad 59$

9.4. Automorphic forms 62

Chapter 10. Smooth $G$-spectra $\quad 65$

10.1. Smooth $G$-sets $\quad 65$

10.2. The category of simplicial smooth $G$-sets $\quad 67$

10.3. The category of smooth $G$-spectra $\quad 69$

10.4. Smooth homotopy fixed points $\quad 70$

10.5. Restriction, induction, and coinduction 71

10.6. Descent from compact open subgroups 72

10.7. Transfer maps and the Burnside category 74

$\begin{array}{ll}\text { Chapter 11. Operations on TAF } & 79\end{array}$

11.1. The $E_{\infty}$-action of $G U\left(\mathbb{A}^{p, \infty}\right) \quad 79$

11.2. Hecke operators $\quad 80$

$\begin{array}{ll}\text { Chapter 12. Buildings } & 87\end{array}$

12.1. Terminology $\quad 87$

12.2. The buildings for $G L$ and $S L \quad 88$

12.3. The buildings for $U$ and $G U \quad 89$

Chapter 13. Hypercohomology of adele groups $\quad 95$

13.1. Definition of $Q_{G U}$ and $Q_{U} \quad 95$

13.2. The semi-cosimplicial resolution $\quad 95$

$\begin{array}{lll}\text { Chapter 14. } K(n) \text {-local theory } & 103\end{array}$

14.1. Endomorphisms of $\bmod p$ points 103

14.2. Approximation results $\quad 105$

14.3. The height $n$ locus of $\operatorname{Sh}\left(K^{p}\right) \quad 108$

14.4. $K(n)$-local TAF 113

14.5. $K(n)$-local $Q_{U} \quad 118$

Chapter 15. Example: chromatic level $1 \quad 121$

15.1. Unit groups and the $K(1)$-local sphere 121

15.2. Topological automorphic forms in chromatic filtration 1

$\begin{array}{lr}\text { Bibliography } & 129\end{array}$

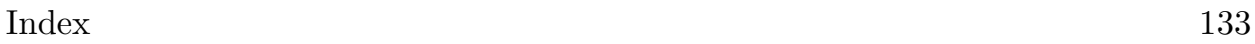




\begin{abstract}
We apply a theorem of J. Lurie to produce cohomology theories associated to certain Shimura varieties of type $U(1, n-1)$. These cohomology theories of topological automorphic forms $(T A F)$ are related to Shimura varieties in the same way that $T M F$ is related to the moduli space of elliptic curves. We study the cohomology operations on these theories, and relate them to certain Hecke algebras. We compute the $K(n)$-local homotopy types of these cohomology theories, and determine that $K(n)$-locally these spectra are given by finite products of homotopy fixed point spectra of the Morava E-theory $E_{n}$ by finite subgroups of the Morava stabilizer group. We construct spectra $Q_{U}(K)$ for compact open subgroups $K$ of certain adele groups, generalizing the spectra $Q(\ell)$ studied by the first author in the modular case. We show that the spectra $Q_{U}(K)$ admit finite resolutions by the spectra $T A F$, arising from the theory of buildings. We prove that the $K(n)$-localizations of the spectra $Q_{U}(K)$ are finite products of homotopy fixed point spectra of $E_{n}$ with respect to certain arithmetic subgroups of the Morava stabilizer groups, which N. Naumann has shown (in certain cases) to be dense. Thus the spectra $Q_{U}(K)$ approximate the $K(n)$-local sphere to the same degree that the spectra $Q(\ell)$ approximate the $K(2)$-local sphere.
\end{abstract}

Received by the editor November 7, 2007.

2000 Mathematics Subject Classification. Primary 55N35; Secondary 55Q51, 55Q45, 11G15.

Key words and phrases. homotopy groups, cohomology theories, automorphic forms, Shimura varieties.

The first author was partially supported by NSF Grant \#0605100, a grant from the Sloan foundation, and DARPA.

The second author was partially supported by NSF Grant \#0402950. 



\section{Introduction}

\subsection{Background and motivation}

The chromatic filtration. Let $X$ be a finite spectrum, and let $p$ be a prime number. The chromatic tower of $X$ is the tower of Bousfield localizations

$$
\cdots \rightarrow X_{E(3)} \rightarrow X_{E(2)} \rightarrow X_{E(1)} \rightarrow X_{E(0)}
$$

with respect to the $p$-primary Johnson-Wilson spectra $E(n)$ (where $E(0)$ is the Eilenberg-MacLane spectrum $H \mathbb{Q}$ ). The chromatic convergence theorem of Hopkins and Ravenel [Rav92] states that the $p$-localization $X_{(p)}$ is recovered by taking the homotopy inverse limit of this tower, and that the homotopy groups of $X_{(p)}$ are given by

$$
\pi_{*} X_{(p)}={\underset{l}{n}}_{n}^{\lim } \pi_{*} X_{E(n)} .
$$

The salient feature of this approach is that the homotopy groups of the monochromatic layers $M_{n} X$, given by the homotopy fibers

$$
M_{n} X \rightarrow X_{E(n)} \rightarrow X_{E(n-1)},
$$

fit into periodic families.

We pause to explain how this works. The periodicity theorem of Hopkins and Smith [HS98] implies that for a cofinal collection of indices $I=\left(i_{0}, \ldots, i_{n}\right)$ there exist finite complexes $M(I)^{0}=M\left(p^{i_{0}}, \ldots, v_{n}^{i_{n}}\right)^{0}$ inductively given by fiber sequences:

$$
M\left(p^{i_{0}}, \ldots, v_{n}^{i_{n}}\right)^{0} \rightarrow M\left(p^{i_{0}}, \ldots, v_{n-1}^{i_{n-1}}\right)^{0} \stackrel{v_{n}^{i_{n}}}{\longrightarrow} \Sigma^{-i_{n}\left|v_{n}\right|} M\left(p^{i_{0}}, \ldots, v_{n-1}^{i_{n-1}}\right)^{0}
$$

Here, $v_{n}^{i_{n}}$ is a $v_{n}$-self map - it induces an isomorphism on Morava $K(n)$-homology. (The 0 superscript is used to indicate that we have arranged for the top cell of these finite complexes to be in dimension 0 .) The $n$th monochromatic layer admits the following alternate description: there is an equivalence

$$
M_{n} X \simeq \underset{I=\left(i_{0}, \ldots, i_{n-1}\right)}{\operatorname{hocolim}} X_{E(n)} \wedge M(I)^{0} .
$$

The homotopy colimit is taken with respect to an appropriate cofinal collection of indices $\left(i_{0}, \ldots, i_{n-1}\right)$. Suppose that

$$
\alpha: S^{k} \rightarrow M_{n} X
$$

represents a non-trivial element of $\pi_{k} M_{n} X$. Then, using (0.1.1), there exists a sequence $I=\left(i_{0}, \ldots, i_{n-1}\right)$ such that $\alpha$ factors as a composite

$$
S^{k} \stackrel{\tilde{\alpha}}{\rightarrow} X_{E(n)} \wedge M(I)^{0} \rightarrow M_{n} X .
$$


Let

$$
v_{n}^{i_{n}}: \Sigma^{i_{n}}\left|v_{n}\right| M(I)^{0} \rightarrow M(I)^{0}
$$

be a $v_{n}$-self map. The map $v_{n}^{i_{n}}$ is an equivalence after smashing with $X_{E(n)}$ this is because $v_{n}^{i_{n}}$ is a $K(n)$-equivalence, and the smash product of an $E(n)$-local spectrum with a type $n$ complex is $K(n)$-local. For each integer $s$, the composite

$$
\alpha_{s}: S^{k+i_{n} s\left|v_{n}\right|} \stackrel{\tilde{\alpha}}{\longrightarrow} \Sigma^{i_{n} s\left|v_{n}\right|} X_{E(n)} \wedge M(I)^{0} \stackrel{\left(v_{n}^{i_{n}}\right)^{s}}{\longrightarrow} X_{E(n)} \wedge M(I)^{0} \rightarrow M_{n} X
$$

gives rise to a family of elements $\alpha_{s} \in \pi_{*} M_{n} X$ which specializes to $\alpha$ for $s=0$.

There are homotopy pullback squares:

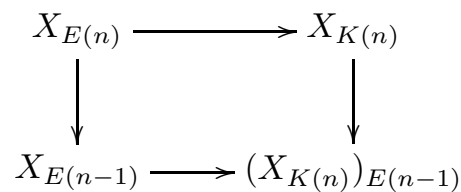

In particular, taking the fibers of the vertical arrows, there is an equivalence $M_{n} X \simeq$ $M_{n}\left(X_{K(n)}\right)$, and understanding the $n$th monochromatic layer of $X$ is equivalent to understanding the $K(n)$-localization of $X$.

The idea of the chromatic tower originates with Jack Morava, who also developed computational techniques for understanding the homotopy groups of $X_{K(n)}$ and $M_{n} X$. For simplicity, let us specialize our discussion to the case where $X$ is the sphere spectrum $S$. Let $E_{n}$ be the Morava $E$-theory spectrum associated to the Honda height $n$ formal group $H_{n}$ over $\overline{\mathbb{F}}_{p}$. It is the Landweber exact cohomology theory whose formal group is the Lubin-Tate universal deformation of $H_{n}$, with coefficient ring

$$
\left(E_{n}\right)_{*}=W\left(\overline{\mathbb{F}}_{p}\right)\left[\left[u_{1}, \ldots, u_{n-1}\right]\right]\left[u^{ \pm 1}\right]
$$

(here $W\left(\overline{\mathbb{F}}_{p}\right)$ is the Witt ring of $\overline{\mathbb{F}}_{p}$ ). Let

$$
\mathbb{G}_{n}=\mathbb{S}_{n} \rtimes \mathrm{Gal}
$$

denote the extended Morava stabilizer group. Here Gal denotes the absolute Galois group of $\mathbb{F}_{p}$, and $\mathbb{S}_{n}=\operatorname{Aut}\left(H_{n}\right)$ is the $p$-adic analytic group of automorphisms of the formal group $H_{n}$. Morava's change of rings theorems state that the Adams-Novikov spectral sequences for $M_{n} S$ and $S_{K(n)}$ take the form:

$$
\begin{aligned}
H_{c}^{*}\left(\mathbb{G}_{n} ;\left(E_{n}\right)_{*} /\left(p^{\infty}, \ldots, u_{n-1}^{\infty}\right)\right) & \Rightarrow \pi_{*} M_{n} S \\
H_{c}^{*}\left(\mathbb{G}_{n} ;\left(E_{n}\right)_{*}\right) & \Rightarrow \pi_{*} S_{K(n)}
\end{aligned}
$$

Thus the homotopy groups of $M_{n} S$ and $S_{K(n)}$ are intimately related to the formal moduli of commutative 1-dimensional formal groups of height $n$.

The action of $\mathbb{G}_{n}$ on the ring $\left(E_{n}\right)_{*}$ is understood [DH95], but is very complicated. The computations of the $E_{2}$-terms of the spectral sequences (0.1.2) and (0.1.3) are only known for small $n$, and even in the case of $n=2$ the computations of Shimomura, Wang, and Yabe [SW02], [SY95], while quite impressive, are difficult to comprehend fully.

Goerss and Hopkins [GH04] extended work of Hopkins and Miller [Rez98] to show that $E_{n}$ is an $E_{\infty}$-ring spectrum, and that the group $\mathbb{G}_{n}$ acts on $E_{n}$ by $E_{\infty}$-ring maps. Devinatz and Hopkins [DH04] gave a construction of continuous homotopy 
fixed points of $E_{n}$ with respect to closed subgroups of $\mathbb{G}_{n}$, and refined Morava's change of rings theorem (0.1.3) to prove that the natural map

$$
S_{K(n)} \stackrel{\simeq}{\longrightarrow} E_{n}^{h \mathbb{G}_{n}}
$$

is an equivalence. This result represents a different sort of computation: the homotopy type of the $K(n)$-local sphere is given as the hypercohomology of the group $\mathbb{G}_{n}$ with coefficients in the spectrum $E_{n}$.

Finite resolutions of $S_{K(n)}$. In [GHMR05], [Hen07], Goerss, Henn, Mahowald, and Rezk proposed a conceptual framework in which to understand the computations of Shimomura, Wang, and Yabe. Namely, they produced finite decompositions of the $K(n)$-local sphere into homotopy fixed point spectra of the form $\left(E_{n}^{h F}\right)^{h G a l}$ for various finite subgroups $F$ of the group $\mathbb{S}_{n}$ ([GHMR05] treats the case $n=2$ and $p=3$, and [Hen07] generalizes this to $n=p-1$ for $p>2$ ). In their work, explicit finite resolutions of the trivial $\mathbb{S}_{n}$-module $\mathbb{Z}_{p}$ by permutation modules of the form $\mathbb{Z}_{p}\left[\left[\mathbb{S}_{n} / F\right]\right]$ are produced. Obstruction theory is then used to realize these resolutions in the category of spectra. These approaches often yield very efficient resolutions. However, the algebraic resolutions are non-canonical. Moreover, given an algebraic resolution, there can be computational difficulties in showing that the obstructions to a topological realization of the resolution vanish.

When the 1-dimensional formal group $H_{n}$ occurs as the formal completion of a 1dimensional commutative group scheme $A$ in characteristic $p$, then global methods may be used to study $K(n)$-local homotopy theory. The formal group is naturally contained in the $p$-torsion of the group scheme $A$. The geometry of the torsion at primes $\ell \neq p$ can be used to produce canonical finite resolutions analogous to those discussed above, as we now describe.

The $K(1)$-local sphere and the $J$ spectrum. In the case of $n=1$, the formal completion of the multiplicative group $\mathbb{G}_{m} / \overline{\mathbb{F}}_{p}$ is isomorphic to the height 1 formal group $H_{1}$. The $J$ spectrum is given by the fiber sequence

$$
J \rightarrow K O_{p} \stackrel{\psi^{\ell}-1}{\longrightarrow} K O_{p}
$$

where $\ell$ is a topological generator of $\mathbb{Z}_{p}^{\times}$(respectively $\mathbb{Z}_{2}^{\times} /\{ \pm 1\}$ if $p=2$ ), and $\psi^{\ell}$ is the $\ell$ th Adams operation. The natural map

$$
S_{K(1)} \stackrel{\simeq}{\rightarrow} J
$$

was shown to be an equivalence by Adams and Baird, and also by Ravenel [Bou79], [Rav84]. The group of endomorphisms

$$
\operatorname{End}\left(\mathbb{G}_{m}\right)[1 / \ell]^{\times}=(\mathbb{Z}[1 / \ell])^{\times}= \pm \ell^{\mathbb{Z}}
$$

is a dense subgroup of the Morava stabilizer group $\mathbb{S}_{1}=\mathbb{Z}_{p}^{\times}$. The $p$-adic $K$-theory spectrum is given by

$$
K O_{p} \simeq\left(E_{1}^{h\{ \pm 1\}}\right)^{h G a l}
$$

and under this equivalence, the fiber sequence (0.1.5) is equivalent to the fiber sequence of homotopy fixed point spectra:

$$
\left(E_{1}^{ \pm \ell^{\mathbb{Z}}}\right)^{h G a l} \rightarrow\left(E_{1}^{h\{ \pm 1\}}\right)^{h G a l} \stackrel{[\ell]-1}{\longrightarrow}\left(E_{1}^{h\{ \pm 1\}}\right)^{h G a l} .
$$


We see that there is an equivalence

$$
J \simeq\left(E_{1}^{h \pm \ell^{\mathbb{Z}}}\right)^{h G a l} .
$$

Equivalence (0.1.6) is then a statement concerning the equivalence

$$
\left(E_{1}^{h \mathbb{S}_{1}}\right)^{h G a l} \stackrel{\simeq}{\longrightarrow}\left(E_{1}^{h \pm \ell^{\mathbb{Z}}}\right)^{h G a l}
$$

of two homotopy fixed point spectra.

The $K(2)$-local sphere and the spectrum $Q(\ell)$. A similar treatment of the case $n=2$ was initiated by Mahowald and Rezk [MR], and carried out further in previous work of the authors. The multiplicative group is replaced with a supersingular elliptic curve $C / \overline{\mathbb{F}}_{p}$. The formal completion of $C$ is isomorphic to the height 2 formal group $H_{2}$. The first author [Beh06] defined a spectrum $Q(\ell)$ to be the totalization of a semi-cosimplicial spectrum arising from analogs of the Adams operations occurring in the theory of topological modular forms:

$$
Q(\ell)=\operatorname{holim}\left(T M F \Rightarrow T M F_{0}(\ell) \times T M F \Rightarrow T M F_{0}(\ell)\right) .
$$

For simplicity, assume that $p$ is odd. The authors [BL06] showed that if $\ell$ is a topological generator of $\mathbb{Z}_{p}^{\times}$, the group

$$
\Gamma=(\operatorname{End}(C)[1 / \ell])^{\times}
$$

is dense in the Morava stabilizer group $\mathbb{S}_{2}$. The first author $[\mathbf{B e h}]$ used the action of $\Gamma$ on the building for $G L_{2}\left(\mathbb{Q}_{\ell}\right)$ to show there is an equivalence

$$
Q(\ell)_{K(2)} \stackrel{\simeq}{\longrightarrow}\left(E_{2}^{h \Gamma}\right)^{h G a l} .
$$

However, the spectrum $Q(\ell)_{K(2)}$ is not equivalent to $S_{K(2)} \simeq\left(E_{2}^{h \mathbb{S}}\right)^{h G a l}$. Rather, the conjecture is that there is a fiber sequence

$$
D_{K(2)} Q(\ell)_{K(2)} \rightarrow S_{K(2)} \rightarrow Q(\ell)_{K(2)} .
$$

This conjecture was verified in [Beh06] in the case of $p=3$ and $\ell=2$.

Periodic families in the stable stems and arithmetic congruences. Miller, Ravenel, and Wilson [MRW77], generalizing the work of Adams, Smith, and Toda, suggested a prominent source of $v_{n}$-periodic families of elements in the stable homotopy groups of spheres. Namely, for $I=\left(p^{i_{0}}, \ldots, v_{n-1}^{i_{n-1}}\right)$, suppose that the associated complex $M(I)^{0}$ admits a $v_{n}$-self map

$$
v_{n}^{i_{n}}: \Sigma^{i_{n}\left|v_{n}\right|} M(I)^{0} \rightarrow M(I)^{0} .
$$

The composite

$$
S^{i_{n} s\left|v_{n}\right|-\|I\|} \stackrel{i}{\rightarrow} \Sigma^{i_{n} s\left|v_{n}\right|} M(I)^{0} \stackrel{\left(v_{n}^{i_{n}}\right)^{s}}{\longrightarrow} M(I)^{0} \stackrel{j}{\rightarrow} S,
$$

where $i$ is the inclusion of the bottom cell, $\|I\|$ is the quantity

$$
\|I\|=i_{1}\left|v_{1}\right|+i_{2}\left|v_{2}\right|+\cdots+i_{n-1}\left|v_{n-1}\right|+n,
$$

and $j$ is the projection onto the top cell, gives the Greek letter element :

$$
\alpha_{s i_{n} / i_{n-1}, \ldots, i_{0}}^{(n)} \in \pi_{*} S .
$$


(This element is not uniquely defined - it depends inductively on the choices of self maps $\left(v_{1}^{i_{1}}, \ldots, v_{n}^{i_{n}}\right)$.) The image of this element in $\pi_{*} S_{E(n)}$ factors through $M_{n} S$ as the composite

$$
S^{i_{n} s\left|v_{n}\right|-\|I\|} \rightarrow \Sigma^{i_{n} s\left|v_{n}\right|} M(I)_{E(n)}^{0} \stackrel{\left(v_{n}^{i_{n}}\right)^{s}}{\longrightarrow} M(I)_{E(n)}^{0} \hookrightarrow M_{n} S .
$$

This composite is necessarily detected in the 0 -line of the spectral sequence 0.1.2 by a non-zero element:

$$
x_{i_{n} / i_{n-1}, \ldots, i_{0}} \in H^{0}\left(\mathbb{G}_{n},\left(E_{n}\right)_{*} /\left(p^{\infty}, \ldots, u_{n-1}^{\infty}\right)\right)
$$

The existence of these invariants determines which sequences $\left(i_{0}, \ldots, i_{n}\right)$ could give rise to a Greek letter element $\alpha_{i_{n} / i_{n-1}, \ldots, i_{0}}^{(n)}$.

There are three important questions one can ask:

QUESTION 0.1.14. For which sequences $\left(i_{0}, \ldots, i_{n}\right)$ do there exist invariants

$$
x_{i_{n} / i_{n-1}, \ldots, i_{0}} \in H^{0}\left(\mathbb{G}_{n},\left(E_{n}\right)_{*} /\left(p^{\infty}, \ldots, u_{n-1}^{\infty}\right)\right) ?
$$

QUESTION 0.1.15. Does there exist a corresponding complex $M(I)^{0}$ and $v_{n}$-self map $v_{n}^{i_{n}}$ ?

QUESTION 0.1.16. If so, is the corresponding Greek letter element $\alpha_{i_{n} / i_{n-1}, \ldots, i_{0}}^{(n)}$ non-trivial?

Question 0.1.14 is a computation, which, as we will describe below, is known for $n=1,2$. We will explain how the $J$-spectrum, in the case $n=1$, and the spectrum $Q(\ell)$ for $n=2$, relate these computations to certain arithmetic congruences. Question 0.1.15 is a very difficult question in computational homotopy theory: it was completely answered for $n=1$ by Adams [Ada66] and Mahowald [Mah70], while even for $p \geq 5$, there are only partial results for $n=2$, due to Smith, Zahler, and Oka (see [Rav86, Sec. 5.5]). Question 0.1.16 is more tractable. For instance, it is completely solved in [MRW77] and [Shi81] for $n=2$ using the chromatic spectral sequence.

The elements $\alpha_{i / j}$ and Bernoulli numbers. Assume that $p>2$. In what follows, let $\nu_{p}$ denote $p$-adic valuation. The homotopy elements $\alpha_{i / j}$ generate the image of the classical $J$ homomorphism. The corresponding invariants $x_{i / j}$ are classified by the following theorem [MRW77, Thm. 4.2].

THEOREM 0.1.17. There exists an invariant

$$
x_{i / j} \in H_{c}^{0}\left(\mathbb{G}_{1},\left(E_{1}\right)_{2 t} /\left(p^{\infty}\right)\right)
$$

of order $p^{j}$ if and only if $t=(p-1) i$ and $j \leq \nu_{p}(i)+1$.

The orders of the generators of these groups of invariants are related to the $p$-adic valuations of denominators of Bernoulli numbers. Let $B_{i}$ denote the $i$ th Bernoulli number.

Lemma 0.1.18 (Lipshitz-Sylvester [MS74, Lem. B.2]). For every pair of integers $k$ and $n$, the quantity $k^{n}\left(k^{n}-1\right) B_{n} / n$ is an integer. 
In particular, if $k$ is chosen to be prime to $p$, then we deduce that

$$
\left(k^{n}-1\right) B_{n} / n
$$

is $p$-integral. If $\ell$ is chosen such that the subgroup $\pm \ell^{\mathbb{Z}}$ is dense in $\mathbb{Z}_{p}^{\times}$, there is an isomorphism

$$
H_{c}^{0}\left(\mathbb{Z}_{p}^{\times},\left(E_{1}\right)_{2 t} /\left(p^{\infty}\right)\right)^{G a l} \stackrel{\cong}{\longrightarrow} H^{0}\left( \pm \ell^{\mathbb{Z}},\left(E_{1}\right)_{2 t} /\left(p^{\infty}\right)\right)^{G a l} .
$$

The sequence (0.1.7) implies that, for $t$ even, there is an exact sequence

$$
0 \rightarrow H^{0}\left( \pm \ell^{\mathbb{Z}},\left(E_{1}\right)_{2 t} /\left(p^{\infty}\right)\right)^{\text {Gal }} \rightarrow \mathbb{Z} / p^{\infty} \stackrel{\ell^{t}-1}{\longrightarrow} \mathbb{Z} / p^{\infty} .
$$

In particular, the image of $B_{t} / t$ in $\mathbb{Z} / p^{\infty}$ is annihilated by $\ell^{t}-1$, and $B_{t} / t$ gives an element $y_{t}$ of $H^{0}\left(\mathbb{G}_{1},\left(E_{1}\right)_{2 t} /\left(p^{\infty}\right)\right)$. In fact, these invariants $y_{t}$ are generators. In summary, there is a correspondence

$$
\alpha_{i / j} \leftrightarrow B_{t} / t \in \mathbb{Q} / \mathbb{Z}_{(p)}
$$

for $t=(p-1) i$ and $j=\nu_{p}(i)+1$.

The elements $\alpha_{i / j}$ and Eisenstein series. We now assume, for simplicity, that $p>3$. We now explain how the Eisenstein series give an alternative approach to the invariants $x_{i / j}$. By explicit calculation, there is an abstract isomorphism

$$
H^{0}\left(\mathbb{G}_{1},\left(E_{1}\right)_{2 t} /\left(p^{\infty}\right)\right) \cong H^{0}\left(\mathbb{G}_{2},\left(E_{2}\left[v_{1}^{-1}\right]\right)_{2 t} /\left(p^{\infty}\right)\right) .
$$

(Presumably this is related to Hopkins' chromatic splitting conjecture.) Choosing $\ell$ as before, the group $\Gamma$ of $(0.1 .10)$ is a dense subgroup of $\mathbb{S}_{2}$, and so there is an isomorphism

$$
H^{0}\left(\mathbb{S}_{2},\left(E_{2}\left[v_{1}^{-1}\right]\right)_{2 t} /\left(p^{\infty}\right)\right)^{G a l} \cong H^{0}\left(\Gamma,\left(E_{2}\left[v_{1}^{-1}\right]\right)_{2 t} /\left(p^{\infty}\right)\right)^{G a l} .
$$

The equivalence (0.1.11) relates this computation to the computation of the equalizers

$$
\mathcal{A}_{t / j} \rightarrow T M F_{2 t} / p^{j} \underset{d_{1}}{\stackrel{d_{0}}{\rightrightarrows}} T M F_{0}(\ell)_{2 t} / p^{j} \oplus T M F_{2 t} / p^{j}
$$

where the maps $d_{0}$ and $d_{1}$ are the maps induced from the semi-cosimplicial coface maps of (0.1.9).

For a $\mathbb{Z}[1 / N]$-algebra $R$, let $M_{t}\left(\Gamma_{0}(N)\right)_{R}$ denote the weight $t$ modular forms for the congruence subgroup $\Gamma_{0}(N)$ defined over the ring $R$. For $p>3$ there are isomorphisms

$$
\begin{aligned}
\left(T M F_{(p)}\right)_{2 t} & \cong M_{t}\left(\Gamma_{0}(1)\right)_{\mathbb{Z}_{(p)}}\left[\Delta^{-1}\right], \\
\left(T M F_{0}(\ell)_{(p)}\right)_{2 t} & \cong M_{t}\left(\Gamma_{0}(\ell)\right)_{\mathbb{Z}_{(p)}}\left[\Delta^{-1}\right] .
\end{aligned}
$$

The coface map $d_{1}$ acts on a modular form $f \in M_{t}\left(\Gamma_{0}(1)\right)_{\mathbb{Z}_{(p)}}\left[\Delta^{-1}\right]$ by

$$
d_{1}(f)=(f, f) \in M_{t}\left(\Gamma_{0}(\ell)\right)_{\mathbb{Z}_{(p)}}\left[\Delta^{-1}\right] \oplus M_{t}\left(\Gamma_{0}(1)\right)_{\mathbb{Z}_{(p)}}\left[\Delta^{-1}\right] .
$$

The coface map $d_{0}$ acts by

$$
d_{0}(f)=\left(\ell^{t} V_{\ell}(f), \ell^{t} f\right)
$$

where, on the level of $q$-expansions, the operator $V_{\ell}$ is given by

$$
\left(V_{\ell}(f)\right)(q)=f\left(q^{\ell}\right) .
$$


Thus, we have

$$
\mathcal{A}_{(t ; j)}=\left\{f \in M_{t}\left(\Gamma_{0}(1)\right)\left[\Delta^{-1}\right] / p^{j}: \quad \begin{array}{ll}
\text { (i) }\left(\ell^{t}-1\right) f \equiv 0 \quad \bmod p^{j} \\
& \text { (ii) } \ell^{t} V_{\ell}(f)-f \equiv 0 \quad \bmod p^{j}
\end{array}\right\} .
$$

Condition (i) implies that $\mathcal{A}_{(t ; j)}$ cannot have elements of order $p^{j}$ unless $t=(p-$ 1) $p^{j-1} s$. If this is satisfied, Condition (ii) on $f$ is equivalent to the assertion that its $q$-expansion satisfies

$$
f(q) \equiv \text { constant } \bmod p^{j} .
$$

The Eisenstein series $E_{t} \in M_{t}\left(\Gamma_{0}(1)\right)_{\mathbb{Z}}$ have $q$-expansions

$$
E_{t}(q)=1-\frac{2 t}{B_{t}}\left(\sum_{i \geq 1} \sigma_{t-1}(i) q^{i}\right)
$$

where

$$
\sigma_{k}(i):=\sum_{d \mid i} d^{k}
$$

Our remarks concerning the $p$-adic valuations of the denominators of $B_{t} / t$ imply that for $j \leq \nu_{p}(i)+1$, the Eisenstein series $E_{(p-1) i}$ are generators of $\mathcal{A}_{(t ; j)}$ of order $p^{j}$. Thus, for $t=(p-1) i$ and $j=\nu_{p}(i)+1$, there is a correspondence

$$
\alpha_{i / j} \leftrightarrow E_{t} \in \mathcal{A}_{(t ; j)} .
$$

REMARK 0.1.19. This technique of computing the 1-line of the Adams-Novikov spectral sequence using Eisenstein series is first seen in the work of A. Baker [Bak99]. Baker uses Hecke operators, and his results may be derived from the discussion above by exploiting the relationship between the Hecke operator $T_{\ell}$ and the verschiebung $V_{\ell}$.

The elements $\beta_{i / j, k}$ and congruences of modular forms. We continue to assume that $p>3$. The sequences $(j, k, i)$ giving invariants $x_{i / j, k}$ corresponding to homotopy elements $\beta_{i / j, k} \in S_{E(2)}$ are completely classified:

Theorem 0.1.20 (Miller-Ravenel-Wilson [MRW77]). The exists an invariant

$$
x_{i / j, k} \in H_{c}^{0}\left(\mathbb{G}_{2},\left(E_{2}\right)_{2 t} /\left(p^{\infty}, v_{1}^{\infty}\right)\right)
$$

of order $p^{k}$ if and only if

(1) $t=\left(p^{2}-1\right) i-(p-1) j$,

(2) $1 \leq j \leq p^{\nu_{p}(i)}+p^{\nu_{p}(i)-1}-1\left(j=1\right.$ if $\left.\nu_{p}(i)=0\right)$,

(3) If $m$ is the unique number satisfying

$$
p^{\nu_{p}(i)-m-1}+p^{\nu_{p}(i)-m-2}-1<j \leq p^{\nu_{p}(i)-m}+p^{\nu_{p}(i)-m-1}-1
$$

then $k \leq \min \left\{\nu_{p}(j)+1, m+1\right\}$.

We will explain how these elaborate conditions on $(j, k, i)$ given in Theorem 0.1.20 reflect a congruence phenomenon occurring amongst $q$-expansions of modular forms. The discussion of congruence properties of Eisenstein series in the last section specializes to give:

$$
E_{p-1}(q) \equiv 1 \quad \bmod p
$$


and thus

$$
E_{p-1}^{p^{k-1}}(q) \equiv 1 \quad \bmod p^{k} .
$$

By the $q$-expansion principle, multiplication by $E_{p-1}^{p^{k-1}}$ gives an injection:

$$
\cdot E_{p-1}^{p^{k-1}}: M_{t}\left(\Gamma_{0}(N)\right)_{\mathbb{Z} / p^{k}} \hookrightarrow M_{t+(p-1) p^{k-1}}\left(\Gamma_{0}(N)\right)_{\mathbb{Z} / p^{k}}
$$

for any $N$ coprime to $p$. In fact, there is a converse:

Theorem 0.1.21 (Serre [Kat73, 4.4.2]). Let $N$ be coprime to $p$. Suppose that for $t_{1}<t_{2}$ we are given modular forms $f_{i} \in M_{t_{i}}\left(\Gamma_{0}(N)\right)_{\mathbb{Z}_{p}}$ whose q-expansions satisfy

$$
f_{1}(q) \equiv f_{2}(q) \quad \bmod p^{k} .
$$

Then $t_{1}$ and $t_{2}$ are congruent modulo $(p-1) p^{k-1}$, and

$$
f_{2} \equiv E_{p-1}^{\frac{t_{2}-t_{1}}{p-1}} \cdot f_{1} \quad \bmod p^{k} .
$$

The Hasse invariant $v_{1} \in M_{p-1}\left(\Gamma_{0}(N)\right)_{\mathbb{F}_{p}}$ lifts to $E_{p-1}$ (see $[$ Kat73, 2.1] — it is necessary that $p>3)$. This allows us to reinterpret the groups $H^{0}\left(\mathbb{G}_{2},\left(E_{2}\right)_{t} /\left(p^{\infty}, u_{1}^{\infty}\right)\right)$ in terms of $p$-adic congruences of modular forms. If $\ell$ is chosen such that the group $\Gamma(0.1 .10)$ is dense in the Morava stabilizer group $\mathbb{S}_{2}$, there is an isomorphism

$$
H^{0}\left(\mathbb{S}_{2},\left(E_{2}\right)_{2 t} /\left(p^{\infty}, u_{1}^{\infty}\right)\right) \cong H^{0}\left(\Gamma,\left(E_{2}\right)_{2 t} /\left(p^{\infty}, u_{1}^{\infty}\right)\right) .
$$

The equivalence (0.1.11), together with the semi-cosimplicial resolution (0.1.9), allow us to deduce that there is an isomorphism

$$
H^{0}\left(\Gamma,\left(E_{2}\right)_{2 t} /\left(p^{\infty}, u_{1}^{\infty}\right)\right)=\underset{\overrightarrow{j, k}}{\lim _{(t ; j, k)}}
$$

where the colimit is taken over pairs $(j, k)$ where $j \equiv 0 \bmod (p-1) p^{k-1}$ and the groups $\mathcal{B}_{(t ; j, k)}$ for such $(j, k)$ are given by the equalizer diagram:

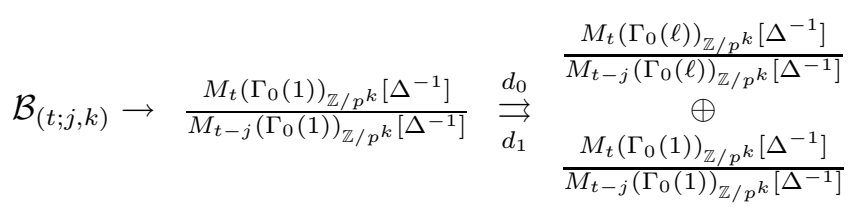

Using our explicit description of the cosimplicial coface maps $d_{0}$ and $d_{1}$, we see that

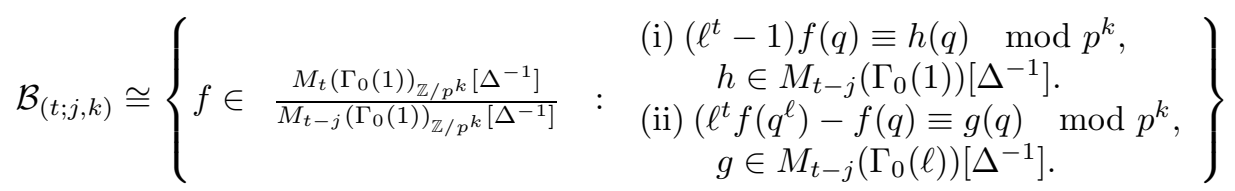

This translates into the following conclusion: there exist level 1 modular forms $f_{t} \in M_{t}\left(\Gamma_{0}(1)\right)\left[\Delta^{-1}\right]$ such that $f_{t}(q)$ is not congruent to any form of lower weight, and such that the existence of $\beta_{i / j, k} \in \pi_{*} S_{E(2)}$ is equivalent to the existence of a congruence

$$
\ell^{t} f\left(q^{\ell}\right)-f(q) \equiv g(q) \quad \bmod p^{k} \quad \text { for } \quad g \in M_{t-j}\left(\Gamma_{0}(\ell)\right)\left[\Delta^{-1}\right] .
$$

for $t=\left(p^{2}-1\right) i$. 
REMARK 0.1.22. In [Lau99] G. Laures defined the $f$ invariant, which gives an embedding of the 2-line of the Adams-Novikov spectral sequence into a ring of divided congruences of modular forms. The authors are unclear at this time how Laures' congruences are related to the congruences described here.

\subsection{Subject matter of this book}

The purpose of this book is to propose entries to the third column of the following table:

\begin{tabular}{c|ccc} 
& $n=1$ & $n=2$ & $n \geq 3$ \\
\hline$E$ & $K O$ & $T M F$ & $?$ \\
$Q$ & $J$ & $Q(\ell)$ & $?$
\end{tabular}

(In fact, our constructions will also specialize to $n=1,2$.) Each of the columns of the table gives spectra $E$ and $Q$, such that:

(1) $E$ is an $E_{\infty}$-ring spectrum associated to a 1 dimensional formal group of height $n$ residing in the formal completion of a commutative group scheme.

(2) The localization $E_{K(n)}$ is a product of homotopy fixed point spectra of $E_{n}$ for finite subgroups of $\mathbb{S}_{n}$.

(3) $Q$ admits a finite semi-cosimplicial resolution by $E_{\infty}$-spectra related to $E$.

(4) The localization $Q_{K(n)}$ is a homotopy fixed point spectrum of $E_{n}$ for an infinite discrete group which is a dense subgroup of $\mathbb{S}_{n}$.

The moduli space of elliptic curves will be replaced by certain PEL Shimura varieties of type $U(1, n-1)$. On first encounter, these moduli spaces seem complicated and unmotivated (at least from the point of view of homotopy theory). There are many excellent references available (for the integral models we are using in this book the reader is encouraged to consult [Kot92], [HT01], and [Hid04]), but these can be initially inaccessible to homotopy theorists. We therefore devote the first few chapters of this book to an expository summary of the Shimura varieties we are considering, singling out only those aspects relevant for our applications to $K(n)$-local homotopy theory.

We briefly summarize the contents of this book.

Topological automorphic forms. To obtain formal groups of height greater than 2 , we must study abelian varieties $A / \overline{\mathbb{F}}_{p}$ of dimension greater than 1 . Since only the moduli of 1-dimensional formal groups seems to be relevant to homotopy theory, we must introduce a device which canonically splits off a 1-dimensional formal summand from the formal completion $\widehat{A}$. This will be accomplished by fixing an imaginary quadratic extension $F$ of $\mathbb{Q}$, with ring of integers $\mathcal{O}_{F}$ in which $p$ splits as $u u^{c}$, and insisting that $A$ admit complex multiplication by $F$, given by a ring homomorphism

$$
i: \mathcal{O}_{F} \hookrightarrow \operatorname{End}(A)
$$


The splitting $F_{p} \cong F_{u} \times F_{u^{c}}$ induces a splitting

$$
\widehat{A} \cong \widehat{A}_{u} \times \widehat{A}_{u^{c}} .
$$

We shall insist that $\widehat{A}_{u}$ have dimension 1 . The other summand $\widehat{A}_{u^{c}}$ will be controlled by means of a compatible polarization - which is given by a certain kind of prime to $p$ isogeny

$$
\lambda: A \rightarrow A^{\vee}
$$

which induces an isomorphism

$$
\lambda_{*}: A(u) \rightarrow A\left(u^{c}\right)^{\vee}
$$

between the $p$-divisible groups which extend the formal groups $\widehat{A}_{u}, \widehat{A}_{u^{c}}^{\vee}$.

Moduli stacks of triples $(A, i, \lambda)$ (over $\mathbb{Z}_{p}$ ) where $A$ has dimension $n$ are given by Shimura stacks $S h$ associated to rational forms of the unitary group $U(1, n-1)$. The formal groups $\widehat{A}_{u}$ associated to points of these Shimura stacks attain heights up to and including height $n$. There are two caveats:

(1) In order to allow for more general forms of $U(1, n-1)$, we will actually fix a maximal order $\mathcal{O}_{B}$ in a central simple algebra $B$ over $F$ of dimension $n^{2}$, which is split at $u$. The algebra $B$ shall be endowed with a positive involution $*$ which restricts to conjugation on $F$. We shall actually consider moduli of triples $(A, i, \lambda)$ where $A$ is an abelian variety of dimension $n^{2}$ with $\mathcal{O}_{B}$-action given by an inclusion of rings

$$
i: \mathcal{O}_{B} \hookrightarrow \operatorname{End}(A)
$$

and $\lambda$ is a compatible polarization. The case of $n$-dimensional polarized abelian varieties with complex multiplication by $F$ is recovered by choosing $B$ to be the matrix algebra $M_{n}(F)$ by Morita equivalence.

(2) Unlike the case of supersingular elliptic curves, there are infinitely many isogeny classes of triples $(A, i, \lambda)$ for which $\widehat{A}_{u}$ has height $n$. Fixing an isogeny class is tantamount to fixing a compatible pairing $\langle-,-\rangle$ on the rank 1 left $B$-module $V=B$. The points in the isogeny class are those points $(A, i, \lambda)$ whose $\lambda$-Weil pairing on the $\ell$-adic Tate modules at each of the places $\ell$ is $B$-linearly similar to $\left(V_{\ell},\langle-,-\rangle\right)$.

For these more general $n^{2}$-dimensional $B$-linear polarized abelian varieties $(A, i, \lambda)$, the formal group $\widehat{A}$ inherits an action of the $p$-complete algebra $B_{p} \cong M_{n}\left(F_{p}\right)$. Fixing a rank 1 projection $\epsilon \in M_{n}\left(F_{p}\right)$ gives a splitting

$$
\widehat{A} \cong\left(\epsilon \widehat{A}_{u} \times \epsilon \widehat{A}_{u^{c}}\right)^{n},
$$

and we insist that the summand $\epsilon \widehat{A}_{u}$ is 1-dimensional.

If the algebra $B$ is a division algebra, then $S h$ possesses an étale projective cover (this is the case considered in [HT01]). The Shimura varieties $S h$ associated to other $B$ need not be compact, but we do not pursue compactifications here.

Lurie has announced an extension of the Goerss-Hopkins-Miller theorem to $p$ divisible groups. Lurie's theorem gives rise to a homotopy sheaf of $E_{\infty}$-ring spectra 
$\mathcal{E}$ on $S h$ in the étale topology. The global sections of $\mathcal{E}$ give rise to a $p$-complete spectrum $T A F$ of topological automorphic forms, and a descent spectral sequence

$$
H^{s}\left(S h, \omega^{\otimes t}\right) \Rightarrow \pi_{2 t-s}(T A F) .
$$

Here, $\omega$ is a line bundle on $S h$ such that over $\mathbb{C}$, the holomorphic sections of $\omega^{\otimes t}$ give rise to certain determinant weight holomorphic automorphic forms.

Hypercohomology of adele groups. Let $\mathbb{A}$ denote the rational adeles. If $S$ is a set of places of $\mathbb{Q}$, we shall use the convention that places in the superscript are omitted and places in the subscript are included:

$$
\begin{aligned}
& \mathbb{A}_{S}=\prod_{v \in S}^{\prime} \mathbb{Q}_{v}, \\
& \mathbb{A}^{S}=\prod_{v \notin S}^{\prime} \mathbb{Q}_{v} .
\end{aligned}
$$

If $S$ is a set of finite rational primes, then this convention naturally extends to the profinite integers:

$$
\begin{aligned}
& \mathbb{Z}_{S}=\prod_{p \in S} \mathbb{Z}_{p}, \\
& \mathbb{Z}^{S}=\prod_{p \notin S} \mathbb{Z}_{p} .
\end{aligned}
$$

This notational philosophy will be extended to other contexts as we see fit.

Let $G U / \mathbb{Q}$ be the algebraic group of similitudes of our fixed pairing $\langle-,-\rangle$ on $V$ :

$$
G U(R)=\left\{g \in \operatorname{Aut}_{B}\left(V \otimes_{\mathbb{Q}} R\right):(g x, g y)=\nu(g)(x, y), \nu(g) \in R^{\times}\right\} .
$$

Let $U$ be the subgroup of isometries. Let $L$ be the $\mathcal{O}_{B}$-lattice in $V=B$ given by the maximal order $\mathcal{O}_{B}$. Let $K_{0}^{p}$ be the compact open subgroup of the group $G U\left(\mathbb{A}^{p, \infty}\right)$ given by

$$
K_{0}^{p}=\left\{g \in G U\left(\mathbb{A}^{p, \infty}\right): g\left(L \otimes \mathbb{Z}^{p}\right)=L \otimes \mathbb{Z}^{p}\right\} .
$$

(Here, $\mathbb{A}^{p, \infty}=\prod_{\ell \neq p}^{\prime} \mathbb{Q}_{\ell}$ is the ring of finite adeles away from $p$, and $\mathbb{Z}^{p}=\prod_{\ell \neq p} \mathbb{Z}_{\ell}$ is the subring of integers.) To each open subgroup $K^{p}$ of $K_{0}^{p}$ one associates an étale cover

$$
\operatorname{Sh}\left(K^{p}\right) \rightarrow S h .
$$

The stack $S h\left(K^{p}\right)$ is the moduli stack of prime-to- $p$ isogeny classes of tuples of the form $\left(A, i, \lambda,[\eta]_{K^{p}}\right)$ where $i$ is a compatible inclusion

$$
i: \mathcal{O}_{B,(p)} \hookrightarrow \operatorname{End}(A)_{(p)}
$$

and

$$
\eta:\left(V \otimes_{\mathbb{Q}} \mathbb{A}^{p, \infty},\langle-,-\rangle\right) \stackrel{\cong}{\longrightarrow}\left(V^{p}(A), \lambda\langle-,-\rangle\right)
$$

represents a $K^{p}$ orbit $[\eta]_{K^{p}}$ of $B$-linear similitudes. Associated to the étale cover $\operatorname{Sh}\left(K^{p}\right)$ is a spectrum $\operatorname{TAF}\left(K^{p}\right)$. Choosing $K^{p}=K_{0}^{p}$ recovers the spectrum TAF.

Associated to the tower of covers $\left\{S h\left(K^{p}\right)\right\}$ (as $K^{p}$ varies over the open subgroups of $\left.K_{0}^{p}\right)$ is a filtered system of $E_{\infty}$-ring spectra $\left\{\operatorname{TAF}\left(K^{p}\right)\right\}$. The colimit

$$
V_{G U}=\underset{K^{p}}{\lim } \operatorname{TAF}\left(K^{p}\right)
$$


admits an action of the group $G U\left(\mathbb{A}^{p, \infty}\right)$ by $E_{\infty}$-ring maps, giving it the structure of a smooth $G$-spectrum. The spectra $T A F\left(K^{p}\right)$ are recovered by taking smooth homotopy fixed points

$$
T A F\left(K^{p}\right) \simeq V_{G U}^{h K^{p}}
$$

More generally, for any set of primes $S$ not containing $p$, and any compact open subgroup $K^{p, S}$ contained in $G U\left(\mathbb{A}^{p, S, \infty}\right)$, we may consider the homotopy fixed point spectrum

where

$$
Q_{G U}\left(K^{p, S}\right):=V_{G U}^{h K_{+}^{p, S}}
$$

$$
K_{+}^{p, S}:=K^{p, S} G U\left(\mathbb{A}_{S}\right) \subseteq G U\left(\mathbb{A}^{p, \infty}\right) .
$$

Letting $G U^{1}\left(\mathbb{A}_{S}\right)$ be the subgroup

$$
G U^{1}\left(\mathbb{A}_{S}\right)=\operatorname{ker}\left(G U\left(\mathbb{A}_{S}\right) \stackrel{\nu}{\rightarrow} \prod_{\ell \in S}^{\prime} \mathbb{Q}_{\ell}^{\times} \stackrel{\nu_{\ell}}{\rightarrow} \prod_{\ell \in S} \mathbb{Z}\right)
$$

of similitudes whose similitude norm has valuation 0 at every place in $S$, it is also convenient to consider the fixed point spectrum

$$
Q_{U}\left(K^{p, S}\right):=V_{G U}^{h K_{1,+}^{p, S}}
$$

where

$$
K_{1,+}^{p, S}:=K^{p, S} G U^{1}\left(\mathbb{A}_{S}\right) \subseteq G U\left(\mathbb{A}^{p, \infty}\right) .
$$

These spectra should be regarded as generalizations of the spectra $Q(\ell)$ to our setting. As the results described in the next section demonstrate, the spectrum $Q_{U}$ seems to be more closely related to the $K(n)$-local sphere than the spectrum $Q_{G U}$.

$K(n)$-local theory. Let $S h\left(K^{p}\right)^{[n]}$ be the (finite) locus of $S h\left(K^{p}\right) \otimes_{\mathbb{Z}_{p}} \mathbb{F}_{p}$ where the formal group $\epsilon \widehat{A}_{u}$ is of height $n$. The $K(n)$-localization of the spectrum $S h\left(K^{p}\right)$ is given by the following theorem.

TheOREm (Corollary 14.5.6). There is an equivalence

$$
\operatorname{TAF}\left(K^{p}\right)_{K(n)} \simeq\left(\prod_{\left(A, i, \lambda,[\eta]_{K^{p}}\right) \in S h\left(K^{p}\right)^{[n]}\left(\overline{\mathbb{F}}_{p}\right)} E_{n}^{h \operatorname{Aut}\left(A, i, \lambda,[\eta]_{K^{p}}\right)}\right)^{h G a l} .
$$

Fix a $\overline{\mathbb{F}}_{p}$-point $\left(A, i, \lambda,[\eta]_{K^{p}}\right)$ of $S h^{[n]}$, and choose a representative $\eta$ of the $K^{p}$-orbit $[\eta]_{K^{p}}$. Let $\Gamma$ be the group of prime-to- $p$ quasi-isometries of $(A, i, \lambda)$.

$$
\Gamma=\left\{\gamma \in \operatorname{End}_{B}(A)_{(p)}^{\times}: \gamma^{\vee} \lambda \gamma=\lambda\right\}
$$

The fixed representative $\eta$ induces an embedding

$$
\Gamma \hookrightarrow G U^{1}\left(\mathbb{A}^{p, S, \infty}\right) .
$$

Let $\Gamma\left(K^{p, S}\right)$ denote the subgroup

$$
\Gamma\left(K^{p, S}\right)=\Gamma \cap K^{p, S} .
$$

The group $\Gamma\left(K^{p, S}\right)$ acts naturally on the formal group

$$
\epsilon \widehat{A}_{u} \cong H_{n}
$$


by isomorphisms, giving an embedding

$$
\Gamma\left(K^{p, S}\right) \hookrightarrow \mathbb{S}_{n} .
$$

We define a spectrum $E\left(\Gamma\left(K^{p, S}\right)\right)$ to be the homotopy fixed point spectrum

$$
E\left(\Gamma\left(K^{p, S}\right)\right)=E_{n}^{h \Gamma\left(K^{p, S}\right)} .
$$

There is a natural map

$$
E_{n}^{h \mathbb{S}_{n}} \rightarrow E\left(\Gamma\left(K^{p, S}\right)\right)
$$

The spectrum $E\left(\Gamma\left(K^{p, S}\right)\right)$ should be regarded as some sort of approximation to the $K(n)$-local sphere. At this time we are unable to even formulate a conjecture (analogous to (0.1.6) in the case of $n=1$ and (0.1.12) in the case of $n=2$ ) quantifying the difference between the spectrum $E\left(\Gamma\left(K^{p, S}\right)\right)$ and the $K(n)$-local sphere. Naumann $[\mathbf{N a u}]$ has proven that in certain circumstances, there exist small sets of primes $S$ such that $\Gamma\left(K^{p, S}\right)$ is of small index in the Morava stabilizer group $\mathbb{S}_{n}$ (see Theorem 14.2.1). The group $\Gamma$ is always dense in $\mathbb{S}_{n}$ (Proposition 14.2.4).

The $K(n)$-localization of the spectrum $Q_{U}\left(K^{p, S}\right)$ is related to Morava $E$-theory by the following theorem.

TheOREM (Corollary 14.5.6). There is an equivalence

$$
Q_{U}\left(K^{p, S}\right)_{K(n)} \simeq\left(\prod_{[g] \in \Gamma \backslash G U^{1}\left(\mathbb{A}^{p, S, \infty}\right) / K^{p, S}} E\left(\Gamma\left(g K^{p, S} g^{-1}\right)\right)\right)^{h G a l} .
$$

This theorem is an analog of (0.1.8) in the case $n=1$, and (0.1.11) in the case of $n=2$.

Building decompositions. For simplicity, we now consider the case where the set $S$ consists of a single prime $\ell \neq p$, where $B$ is split over all of the places that divide $\ell$. The group $\Gamma\left(K^{p, \ell}\right)$ sits naturally inside the group $U\left(\mathbb{Q}_{\ell}\right)$ through its action on the Tate module $V_{\ell}(A)$ through $B$-linear isometries. Let $\mathcal{B}(U)$ be the BruhatTits building for $U\left(\mathbb{Q}_{\ell}\right)$. The finite dimensional complex $\mathcal{B}(U)$ is contractible, and the group $U\left(\mathbb{Q}_{\ell}\right)$ acts on it with compact open stabilizers. This action extends naturally to an action of the group $G U^{1}\left(\mathbb{Q}_{\ell}\right)$.

THEOREM (Proposition 14.1.2). The group $\Gamma\left(K^{p, \ell}\right)$ acts on $\mathcal{B}(U)$ with finite stabilizers, and these stabilizers are the automorphism groups of points of $\operatorname{Sh}\left(K^{p}\right)^{[n]}\left(\overline{\mathbb{F}}_{p}\right)$ for various compact open subgroups $K^{p}$ of $G U^{1}\left(\mathbb{A}^{p, \infty}\right)$.

Therefore, the virtual cohomological dimension of $\Gamma\left(K^{p, \ell}\right)$ is equal to the dimension of $\mathcal{B}(U)$. We have

$$
\operatorname{dim} \mathcal{B}(U)= \begin{cases}n & \ell \text { splits in } F \\ n / 2 \text { or }(n-2) / 2 & \ell \text { does not split in } F, n \text { even, } \\ (n-1) / 2 & \ell \text { does not split in } F, n \text { odd }\end{cases}
$$


We see that the virtual cohomological dimension of $\Gamma\left(K^{p, \ell}\right)$ is maximized when $\ell$ splits in $F$. In this case, there is an isomorphism

$$
U\left(\mathbb{Q}_{\ell}\right) \cong G L_{n}\left(\mathbb{Q}_{\ell}\right)
$$

and the building $\mathcal{B}(U)$ is especially easy to understand. Even in the split case, unless $n=1$, the map (0.2.2) cannot be an equivalence, because $\Gamma\left(K^{p, \ell}\right)$ has virtual cohomological dimension $n$, whereas $\mathbb{S}_{n}$ has virtual cohomological dimension $n^{2}$.

The action of $G U^{1}\left(\mathbb{Q}_{\ell}\right)$ on $\mathcal{B}(U)$ gives rise to the following theorem.

Theorem (Theorem 13.2.9). There is a semi-cosimplicial spectrum $Q_{U}\left(K^{p, \ell}\right)^{\bullet}$ of length $d=\operatorname{dim} \mathcal{B}(U)$ whose sth term $(0 \leq s \leq d)$ is given by

$$
Q_{U}\left(K^{p, \ell}\right)^{s}=\prod_{[\sigma]} T A F(K(\sigma)) .
$$

The product ranges over $U\left(\mathbb{Q}_{\ell}\right)$ orbits of s-simplices $[\sigma]$ in the building $\mathcal{B}(U)$. The groups $K(\sigma)$ are given by

$$
K(\sigma)=K^{p} K_{\ell}(\sigma)
$$

where $K_{\ell}(\sigma)$ is the subgroup of $G U^{1}\left(\mathbb{Q}_{\ell}\right)$ which stabilizes $\sigma$. There is an equivalence

$$
Q_{U}\left(K^{p, \ell}\right) \simeq \operatorname{Tot} Q_{U}\left(K^{p, \ell}\right)^{\bullet} .
$$

REMARK 0.2.5. Theorem 13.2.9 gives a similar semi-cosimplicial resolution of the spectrum $Q_{G U}\left(K^{p, \ell}\right)$.

The spectra $Q_{U}\left(K^{p, \ell}\right)$ should be regarded as a height $n$ analog of the $J$-theory spectrum. The approach taken in this paper towards defining $Q_{U}\left(K^{p, \ell}\right)$ is different from that taken in defining the spectra $J$ and $Q(\ell)$. The spectra $J$ and $Q(\ell)$ were defined as the totalization of an appropriate semi-cosimplicial spectrum (0.1.5), (0.1.9). Here, we define $Q_{U}\left(K^{p, \ell}\right)$ to be a hypercohomology spectrum, and then show that it admits a semi-cosimplicial decomposition.

Potential applications to Greek letter elements. We outline some potential applications the spectra TAF and $Q_{U}$ could have in the study of Greek letter elements in chromatic filtration greater than 2. Specifically, we will discuss the applicability of our methods towards resolving Questions 0.1.14 and 0.1.16. We do not anticipate our constructions to be relevant towards the resolution of Question 0.1.15.

Existence of $x_{i_{n} / i_{n-1}, \ldots, i_{0}}$. Suppose that the set of primes $S$ and the compact open subgroup $K^{p, S}$ are chosen such that the corresponding subgroup

$$
\Gamma\left(K^{p, S}\right) \subset \mathbb{S}_{n}
$$

is dense. For instance, $S$ may consist of a single prime $\ell$ (see Theorem 14.2.1) or $S$ may always be taken to consist of all primes different from $p$ (see Proposition 14.2.4). Then the natural map

$$
H^{0}\left(\mathbb{S}_{n},\left(E_{n}\right)_{2 t} /\left(p^{\infty}, \ldots, u_{n-1}^{\infty}\right)\right) \rightarrow H^{0}\left(\Gamma\left(K^{p, S}\right),\left(E_{n}\right)_{2 t} /\left(p^{\infty}, \ldots, u_{n-1}^{\infty}\right)\right)
$$

is an isomorphism.

Suppose that $S$ consists of a single prime $\ell$ which splits in $F$. Then we have $U\left(\mathbb{Q}_{\ell}\right) \cong G L_{n}\left(\mathbb{Q}_{\ell}\right)$. The equivalences $(0.2 .1),(0.2 .3)$, and $(0.2 .4)$ may be combined 
to show that the existence of $x_{i_{n} /\left(i_{n-1}, \ldots, i_{0}\right)}$ is, under suitable hypotheses, detected in the equalizer of the maps

$$
\pi_{2 t} M_{n} T A F\left(K^{p, \ell} K_{\ell}\right) \underset{d_{1}}{\stackrel{d_{0}}{\rightrightarrows}} \prod_{[\sigma]} \pi_{2 t} M_{n} T A F\left(K^{p, \ell} K_{\ell}(\sigma)\right) .
$$

Here $M_{n}$ denotes the $n$th monochromatic fiber, $K_{\ell} \subset G L_{n}\left(\mathbb{Q}_{\ell}\right)$ is a stabilizer of the unique orbit of 0-simplices in the building $\mathcal{B}\left(G L_{n}\left(\mathbb{Q}_{\ell}\right)\right)$, and the product ranges over the orbits $[\sigma]$ of 1 -simplices in $\mathcal{B}\left(G L_{n}\left(\mathbb{Q}_{\ell}\right)\right)$, with stabilizers $K_{\ell}(\sigma) \subset$ $G L_{n}\left(\mathbb{Q}_{\ell}\right)$. It is our hope that this relationship will lead to a description of the invariants $x_{i_{n} / i_{n-1}, \ldots, i_{0}}$ (Question 0.1.14) related to arithmetic properties of automorphic forms analogous to the descriptions for $n=1,2$ given in Section 0.1.

Non-triviality of Greek letter elements. Miller, Ravenel, and Wilson [MRW77] construct Greek letter elements in the $n$-line of the Adams-Novikov spectral sequence in the following manner. There is a composition of connecting homomorphisms

$$
\partial_{n}: H^{0}\left(\mathbb{G}_{n},\left(E_{n}\right)_{*} /\left(p^{\infty}, \ldots, u_{n-1}^{\infty}\right)\right) \rightarrow \operatorname{Ext}_{B P_{*} B P}^{n, *}\left(B P_{*}, B P_{*}\right) .
$$

Given $x_{i_{n} / i_{n-1}, \ldots, i_{0}} \in H^{0}\left(\mathbb{G}_{n},\left(E_{n}\right)_{*} /\left(p^{\infty}, \ldots, u_{n-1}^{\infty}\right)\right)$, its image under $\partial_{n}$ is the element in the Adams-Novikov spectral sequence which will detect the corresponding Greek letter element $\alpha_{i_{n} / i_{n-1}, \ldots, i_{0}}^{(n)}$ if it exists. Question 0.1.16 is answered in the affirmative if

$$
\partial_{n}\left(x_{i_{n} / i_{n-1}, \ldots, i_{0}}\right) \neq 0 .
$$

Because the length of the semi-cosimplicial resolution of $Q(\ell)$ is $n$, there is a corresponding sequence of boundary homomorphisms for the spectrum $Q_{U}\left(K^{p, \ell}\right)$, where Question 0.1.16 may have a more tractable solution.

\subsection{Organization of this book}

Many chapters of this book are expository in nature, and are meant to serve as a convenient place for the reader who is a homotopy theorist to assimilate the necessary background information in a motivated and direct manner.

In Chapter 1 we briefly describe the theory of $p$-divisible groups, and recall their classification up to isogeny.

In Chapter 2 we review the Honda-Tate classification of abelian varieties up to isogeny over $\overline{\mathbb{F}}_{p}$. Our aim is to establish that there is an obvious choice of isogeny class which supplies height $n$ formal groups of dimension 1 . We explain the generalization to $B$-linear abelian varieties.

In Chapter 3 we describe the notion of a level structure, and explain how homomorphisms of abelian varieties and abelian schemes may be understood through the Tate representation.

In Chapter 4 we introduce the notion of a polarization of an abelian variety, and its associated Weil pairing on the Tate module. We review the classification of polarizations up to isogeny, which is essentially given by the classification of certain alternating forms on the Tate module. 
In Chapter 5 we explain some basic facts about alternating and hermitian forms, and their groups of isometries and similitudes. We summarize the classification of alternating forms. This classification completes the classification of polarizations, and is subsequently applied to the analysis of the height $n$ locus $S h^{[n]}$ of $S h$.

The classification of polarized abelian varieties up to isogeny suggests a natural moduli problem to study, which we explain in Chapter 6. There are two equivalent formulations of the moduli problem. The moduli problem is representable by the Shimura stack $S h$.

In Chapter 7, we summarize the Grothendieck-Messing theory of deformations of $p$-divisible groups. We then explain how this is related to the deformation theory of abelian varieties via Serre-Tate theory. This machinery is then applied to understand the deformation theory of $\bmod p$ points of $S h$.

In Chapter 8, we summarize Lurie's generalization of the Hopkins-Miller theorem. We then use the deformation theory to apply this theorem to our Shimura stacks to define spectra of topological automorphic forms.

Chapter 9 summarizes the classical holomorphic automorphic forms associated to $S h$, and explains the relationship to topological automorphic forms.

In Chapter 10, we pause to rapidly develop the notion of a smooth $G$-spectrum for $G$ a locally compact totally disconnected group. We define smooth homotopy fixed points, and study the behavior of this construction under restriction and coinduction. We prove that a smooth $G$-spectrum is determined up to equivalence by its homotopy fixed points for compact open subgroups of $G$. We define transfer maps and observe that the homotopy fixed points of a smooth $G$-spectrum define a Mackey functor from a variant of the Burnside category to the stable homotopy category.

In Chapter 11 we describe $E_{\infty}$-operations on $T A F\left(K^{p}\right)$ given by elements of the group $G U\left(\mathbb{A}^{p, \infty}\right)$. This gives the data to produce a smooth $G U\left(\mathbb{A}^{p, \infty}\right)$-spectrum $V_{G U}$. We then describe how this structure extends to make the functor TAF(-) a Mackey functor with values in the stable homotopy category. We discuss the obstructions to deducing that the Hecke algebra for the pair $\left(G U\left(\mathbb{A}^{p, \infty}\right), K^{p}\right)$ acts on $T A F\left(K^{p}\right)$ through cohomology operations, and give necessary conditions for these obstructions to vanish.

In Chapter 12, we recall explicit lattice chain models for the buildings for the groups $G U\left(\mathbb{Q}_{\ell}\right)$ and $U\left(\mathbb{Q}_{\ell}\right)$.

In Chapter 13, the spectra $Q_{G U}$ and $Q_{U}$ are defined. We then describe the semicosimplicial resolution of $Q_{U}$.

In Chapter 14, we describe the height $n$ locus $S h^{[n]}$. We then relate the $K(n)$ localization of $T A F$ and $Q_{U}$ to fixed point spectra of Morava $E$-theory.

In Chapter 15, we study the example of $n=1$. In this case the spectra TAF are easily described as products of fixed points of the $p$ completion of the complex $K$ theory spectrum. The spectrum $Q_{U}\left(K^{p, \ell}\right)$ is observed to be equivalent to a product 
of $J$-spectra for $F$ and $\ell$ chosen suitably well. The reader might find it helpful to look ahead to this section to see how the theory plays out in this particular context.

\subsection{Acknowledgments}

The cohomology theory TAF studied in this book is not the first generalization of $T M F$ to higher chromatic levels. Homotopy fixed points $E O_{n}$ of $E_{n}$ by finite subgroups of the Morava stabilizer group were introduced by Hopkins and Miller, and have been studied by Hill, Mahowald, Nave, and others. In certain instances, the relationship of these spectra to moduli spaces arising in algebraic geometry have also been considered by Hopkins, Gorbounov, Mahowald and Lurie in the case of $n=p-1$, and Ravenel in more general cases.

The idea of associating cohomology theories to Shimura varieties also does not originate with the authors - Goerss, Lurie, and Morava have considered such matters. The authors are especially grateful to Jacob Lurie, who generously shared his work and results with the authors.

The authors benefited greatly from conversations with Frank Calegari, Daniel Davis, Johan de Jong, Paul Goerss, Mike Hopkins, Haynes Miller, Catherine O'Neil, and Akshay Venkatesh. The authors would like to thank Niko Naumann for his comments, his observant corrections, and for keeping us informed of his timely work. The first author also expresses his gratitude to Robert Kottwitz, for both introducing him to the subject of Shimura varieties, and sharing his time and knowledge.

Mark Behrens

Tyler Lawson 



\section{CHAPTER 1}

\section{p-divisible groups}

If $A$ is an $n$-dimensional abelian variety over a field $k$, then it has a group $A\left[p^{\infty}\right]$ of $p$-torsion points defined over an algebraic closure $\bar{k}$. If $k$ has characteristic distinct from $p$, this is a group abstractly isomorphic to $\left(\mathbb{Z} / p^{\infty}\right)^{2 n}$ with an action of the Galois group of $k$.

However, if $p$ divides the characteristic of $k$, this breaks down. For example, an elliptic curve over a finite field can either be ordinary (having $p$-torsion points isomorphic to the group $\mathbb{Z} / p^{\infty}$ ) or supersingular (having no non-trivial $p$-torsion points whatsoever). In these cases, however, these missing torsion points appear in the formal group of the abelian variety. If the elliptic curve is ordinary, the formal group has height 1, whereas if the elliptic curve is supersingular, the formal group has height 2. The missing rank in the $p$-torsion arises as height in the formal group.

The correct thing to do in this context is to instead consider the inductive system of group schemes given by the kernels of the homomorphism

$$
\left[p^{i}\right]: A \rightarrow A \text {. }
$$

The ind-finite group schemes that arise are called $p$-divisible groups.

\subsection{Definitions}

Definition 1.1.1. A p-divisible group of height $h$ over a scheme $S$ is a sequence of commutative group schemes over $S$

$$
\{1\}=\mathbb{G}_{0} \hookrightarrow \mathbb{G}_{1} \hookrightarrow \mathbb{G}_{2} \hookrightarrow \cdots
$$

such that each $\mathbb{G}_{i}$ is locally free of rank $p^{i h}$ over $S$, and such that for each $i \geq 0$ the sequence

$$
0 \rightarrow \mathbb{G}_{i} \hookrightarrow \mathbb{G}_{i+1} \stackrel{\left[p^{i}\right]}{\longrightarrow} \mathbb{G}_{i+1}
$$

is exact.

If $S$ is affine, this corresponds to a pro-system $\left\{R_{i}\right\}$ of bicommutative Hopf algebras over a ring $R$, with $R_{i}$ finitely generated projective of rank $p^{i h}$.

EXAMPLE 1.1.2. Any finite group $G$ gives rise to a finite group scheme $G_{S}$ over $S$

$$
G_{S}=\coprod_{G} S .
$$

The inductive system $\left\{\left(\left(\mathbb{Z} / p^{i}\right)^{h}\right)_{S}\right\}$ gives a $p$-divisible group $\left(\left(\mathbb{Z} / p^{\infty}\right)^{h}\right)_{S}$ of height $h$. 
EXAMPLE 1.1.3. Let $R$ be a $p$-complete ring, and let $\mathbb{G}$ be a formal group over $R$. Let $\overline{\mathbb{G}}$ denote the reduction of $\mathbb{G}$ to $k=R /(p)$, and assume that $\overline{\mathbb{G}}$ has constant and finite height $h$. The the $p$-series of $\overline{\mathbb{G}}$ takes the form

$$
[p]_{\mathbb{G}}(x)=u x^{p^{h}}+\cdots \quad \bmod p
$$

with $u \in k^{\times}$. The $p^{i}$-series of $\overline{\mathbb{G}}$ is then given by

$$
\left[p^{i}\right]_{\mathbb{G}}(x)=u^{\prime} x^{p^{i h}}+\cdots \bmod p
$$

for $u^{\prime} \in k^{\times}$. We deduce, using Weierstrass preparation, that the ring of functions of the formal group $\mathbb{G}$ is given by

$$
\mathcal{O}_{\mathbb{G}}=R[[x]]=\lim _{i} R[[x]] /\left(\left[p^{i}\right]_{\mathbb{G}}(x)\right) .
$$

Therefore, $\mathbb{G}$ may be regarded as a $p$-divisible group of height $h$ over $\operatorname{Spec}(R)$.

EXAMPLE 1.1.4. If $A / S$ is an abelian scheme of dimension $n$, the inductive system of kernels $\left\{A\left[p^{i}\right]\right\}$ of the $p^{i}$ th power maps forms a $p$-divisible group of height $2 n$, which we denote by $A(p)$.

EXAMPle 1.1.5. Suppose $E$ is an even periodic $p$-complete ring spectrum. The spectrum $E$ is complex orientable, and so associated to $E$ we have a formal group law $\mathbb{G}_{E}$ over $E^{0}$, represented by the complete Hopf algebra $E^{0}\left(\mathbb{C P}^{\infty}\right) \cong E^{0}[[x]]$. Assume that the height of the mod $p$ reduction of $\mathbb{G}_{E}$ is constant and finite. Then Example 1.1.3 implies that taking Spec of the successive quotients $R_{i}=$ $E^{0}[[x]] /\left(\left[p^{i}\right]_{\mathbb{G}_{E}}(x)\right)$ gives a $p$-divisible group over $E^{0}$. The homotopy equivalence

$$
\operatorname{holim}\left(\Sigma_{+}^{\infty} B \mu_{p^{k}}\right)_{p}^{\wedge} \rightarrow\left(\Sigma_{+}^{\infty} B S^{1}\right)_{p}^{\wedge}
$$

allows us to express the Hopf algebra $E^{0}\left(\mathbb{C P}^{\infty}\right)$ as a limit of the Hopf algebras $E^{0}\left(B \mu_{p^{i}}\right)=R_{i}$ (see, for example, [Sad92, 2.3.1]).

Definition 1.1.6. A homomorphism of $p$-divisible groups $f: \mathbb{G} \rightarrow \mathbb{G}^{\prime}$ is a compatible sequence of homomorphisms

$$
f_{i}: \mathbb{G}_{i} \rightarrow \mathbb{G}_{i}^{\prime} .
$$

The homomorphism $f$ is an isogeny if there exists a homomorphism $f^{\prime}: \mathbb{G}^{\prime} \rightarrow \mathbb{G}$ and an integer $k$ such that $f f^{\prime}=\left[p^{k}\right]$ and $f^{\prime} f=\left[p^{k}\right]$.

If $\mathbb{G}$ is a $p$-divisible group over $S$, it has a Cartier dual

$$
\mathbb{G}^{\vee}=\operatorname{Hom}_{\text {grp.schm } / S}\left(\mathbb{G}, \mathbb{G}_{m}\right) .
$$

If $S=\operatorname{Spec}(R)$ and $\mathbb{G}_{i}=\operatorname{Spec}\left(R_{i}\right),\left(\mathbb{G}^{\vee}\right)_{i}$ corresponds to the dual Hopf algebroid $\operatorname{Hom}_{R}\left(R_{i}, R\right)$. The factorization

$$
\mathbb{G}_{i} \stackrel{[p]}{\longrightarrow} \mathbb{G}_{i-1} \hookrightarrow \mathbb{G}_{i}
$$

of the $p$ th power map dualizes to a sequence

$$
\mathbb{G}_{i}^{\vee} \hookleftarrow \mathbb{G}_{i-1}^{\vee} \stackrel{[p]}{\longleftarrow} \mathbb{G}_{i}^{\vee} .
$$

The left-hand maps make the family $\left\{\mathbb{G}_{i}^{\vee}\right\}$ into a $p$-divisible group. There is a natural isomorphism $\mathbb{G} \rightarrow\left(\mathbb{G}^{\vee}\right)^{\vee}$, so $\vee$ is a anti-equivalence of the category of p-divisible groups. 


\subsection{Classification}

A $p$-divisible group is simple if it is not isogenous to a non-trivial product of $p$ divisible groups. If $\mathbb{G}$ is a simple $p$-divisible group over a field $k$ of characteristic $p$, there is a short exact sequence

$$
0 \rightarrow \mathbb{G}^{0} \rightarrow \mathbb{G} \rightarrow \mathbb{G}^{e t} \rightarrow 0,
$$

where $\left(\mathbb{G}^{0}\right)_{i}$ is connected and $\left(\mathbb{G}^{e t}\right)_{i}$ is étale over $k$ [Tat67]. The dimension of $\mathbb{G}$ is the dimension of the formal group $\mathbb{G}^{0}$. Height and dimension are both additive in short exact sequences and preserved by isogeny. A $p$-divisible group is classified up to isogeny by this data.

TheOREm 1.2.1 ([Dem72], [Mil79]). Let $k$ be an algebraically closed field of characteristic $p$.

(1) Any p-divisible group over $k$ is isogenous to a product $\prod \mathbb{G}_{i}$ of simple p-divisible groups, unique up to permutation.

(2) Simple p-divisible groups $\mathbb{G}$ over $k$ are determined up to isogeny by a pair of relatively prime integers $(d, h), 0 \leq d \leq h$, where $d$ is the dimension of $\mathbb{G}$ and $h \geq 1$ is the height. The fraction $0 \leq \frac{d}{h} \leq 1$ is called the slope of the p-divisible group.

(3) The dual of such a p-divisible group $\mathbb{G}$ has height $h$ and dimension $h-d$. Thus the slope of $\mathbb{G}^{\vee}$ is $1-\frac{d}{h}$.

(4) The endomorphism ring $\operatorname{End}(\mathbb{G})$ is the unique maximal order in the central division algebra $D$ over $\mathbb{Q}_{p}$ of invariant $\frac{d}{h}$.

The isogeny class of a $p$-divisible group is often represented by its Newton polygon. One draws a graph in which the horizontal axis represents total height, and the vertical axis represents total dimension. Each simple summand of dimension $d$ and height $h$ is represented by a line segment of slope $\frac{d}{h}$, with the line segments arranged in order of increasing slope.

The following is a Newton polygon of a $p$-divisible group of height 6 and dimension 3 , with simple summands of slope $\frac{1}{2}, \frac{1}{3}$, and 1 .

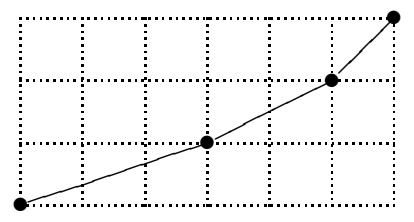

If $A$ is an abelian variety, the $p$-divisible group of the dual abelian variety $A^{\vee}$ is given by $A^{\vee}(p)=(A(p))^{\vee}$. Every abelian variety $A$ over a field admits a polarization, which is a certain type of isogeny $\lambda: A \rightarrow A^{\vee}$ (see Chapter 4, in particular Remark 4.1.6). This forces the $p$-divisible group $A(p)$ to have a symmetry condition: if $\lambda$ is a slope appearing $m$ times in $A(p)$, so is $1-\lambda$.

EXAMPLE 1.2.2. There are two possible Newton polygons associated to elliptic curves, corresponding to the supersingular and ordinary types. 


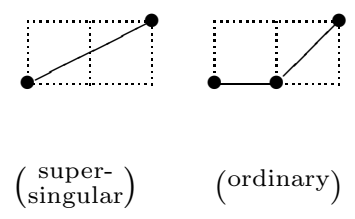

Suppose that $\mathbb{G} / S$ is a $p$-divisible group, $F$ is a finite extension field of $\mathbb{Q}$ with ring of integers $\mathcal{O}_{F}$, and $i: \mathcal{O}_{F} \rightarrow \operatorname{End}(\mathbb{G})$ is a ring homomorphism. Then the map $i$ factors through the $p$-completion of $\mathcal{O}_{F}$, and gives rise to an associated map

$$
i_{p}: \mathcal{O}_{F, p} \rightarrow \operatorname{End}(\mathbb{G}) .
$$

Let $\left\{u_{i}\right\}$ be the primes dividing $p$; then $\mathcal{O}_{F, p} \cong \prod \mathcal{O}_{F, u_{i}}$. In particular, the completion contains idempotents $e_{i}$ whose images in $\operatorname{End}(\mathbb{G})$ give a direct sum decomposition

$$
\mathbb{G} \cong \bigoplus_{i} \mathbb{G}\left(u_{i}\right)
$$

If $A$ is an abelian variety with a map $\mathcal{O}_{F} \rightarrow \operatorname{End}(A)_{(p)}$ and $u$ is a prime of $F$ dividing $p$, we write $A(u)$ for the corresponding summand of its $p$-divisible group. 


\section{CHAPTER 2}

\section{The Honda-Tate classification}

In this chapter we will state the classification, due to Honda and Tate [Hon68], [Tat71], of abelian varieties over $\mathbb{F}_{p^{r}}$, and over $\overline{\mathbb{F}}_{p}$.

\subsection{Abelian varieties over finite fields}

Define the set of quasi-homomorphisms between abelian varieties $A$ and $A^{\prime}$ to be the rational vector space

$$
\operatorname{Hom}^{0}\left(A, A^{\prime}\right)=\operatorname{Hom}\left(A, A^{\prime}\right) \otimes \mathbb{Q} .
$$

(The set $\operatorname{Hom}\left(A, A^{\prime}\right)$ is a finitely generated free abelian group.) Let $\mathrm{AbVar}_{k}^{0}$ denote the category whose objects are abelian varieties over a field $k$, and whose morphisms are the quasi-homomorphisms. A quasi-isogeny is an isomorphism in $\mathrm{AbVar}_{k}^{0}$. An isogeny is a quasi-isogeny which is an actual homomorphism.

A proof of the following theorem may be found in [Mum70, IV.19].

TheOREM 2.1.1. The category $\mathrm{AbVar}_{k}^{0}$ is semisimple.

Corollary 2.1.2. For an abelian variety $A \in \mathrm{AbVar}_{k}^{0}$, the endomorphism ring $\operatorname{End}^{0}(A)$ is semisimple.

Suppose that $A$ is a simple abelian variety over $\mathbb{F}_{q}$, where $q=p^{r} . A$ admits a polarization $\lambda: A \rightarrow A^{\vee}$ (Chapter 4$)$. The quasi-endomorphism $\operatorname{ring} E=\operatorname{End}^{0}(A)$ is of finite dimension over $\mathbb{Q}$. Because $A$ is simple, this is also a division algebra. The polarization $\lambda$ induces a Rosati involution $*$ on $E$ (see Section 4.2 ). Let $M$ be the center of $E$, and let $m$ be given by

$$
[E: M]=m^{2} .
$$

The field $M$ is a finite extension of $\mathbb{Q}$. Let $d$ be the degree $[M: \mathbb{Q}]$.

The involution $*$ restricts to an involution $c$ on $M$. Because the Rosati involution is given by conjugation with the polarization, $c$ is independent of the choice of polarization $\lambda$. Let $M^{+}$be the subfield of $M$ fixed by $c$. The involution $*$ satisfies a positivity condition that implies that $M^{+}$must be totally real, and if $M \neq M^{+}$, then $M$ is a totally imaginary quadratic extension of $M^{+}$. In other words, $M$ is a CM field.

Because $A$ is defined over $\mathbb{F}_{q}$, it has a canonical Frobenius endomorphism $\pi \in$ $\operatorname{End}(A) \cap M$. The $\operatorname{ring} \operatorname{End}(A)$ is finitely generated over $\mathbb{Z}$, and so $\pi$ must be in the ring of integers $\mathcal{O}_{M}$. 
Theorem 2.1.3 (Weil, Honda-Tate).

(1) The field $M$ is generated by $\pi$ over $\mathbb{Q}$.

(2) The algebraic integer $\pi$ is a Weil $q$-integer, i.e. has absolute value $q^{1 / 2}$ in any complex embedding $M \hookrightarrow \mathbb{C}$.

(3) The local invariants of $E$ at primes $x$ of $M$ are given by

$$
\begin{aligned}
\operatorname{inv}_{x} E & =1 / 2, & & x \text { real }, \\
\operatorname{inv}_{x} E & =0, & & x \nmid p, \\
\operatorname{inv}_{x} E & =\frac{x(\pi)}{x(q)}\left[M_{x}: \mathbb{Q}_{p}\right], & & x \mid p .
\end{aligned}
$$

where $x(-)$ denotes the valuation associated to a prime $x$.

(4) The dimension of $A$ satisfies $2 \operatorname{dim}(A)=d \cdot m$, and $m$ is the least common denominator of the $\operatorname{inv}_{x} E$.

(5) The natural map

$$
\left\{\text { quasi-isogeny classes of simple abelian varieties over } \mathbb{F}_{q}\right\}
$$

$$
\{\text { Weil q-integers }\} /\left\{\pi \sim i\left(\pi^{\prime}\right) \text { for any } i: M \hookrightarrow M^{\prime}\right\}
$$

is a bijection.

(6) For abelian varieties $A$ and $B$ and any prime $\ell$, the map

$$
\operatorname{Hom}_{\mathbb{F}_{q}}(A, B)_{\ell} \rightarrow \operatorname{Hom}_{G a l\left(\overline{\mathbb{F}}_{q} / \mathbb{F}_{q}\right)}(A(\ell), B(\ell))
$$

is an isomorphism.

The inclusion map $\mathbb{F}_{q} \rightarrow \mathbb{F}_{q^{r}}$ induces an extension-of-scalars map

$$
A \mapsto A \underset{\operatorname{Spec}\left(\mathbb{F}_{q}\right)}{\times} \operatorname{Spec}\left(\mathbb{F}_{q^{r}}\right),
$$

which preserves quasi-isogenies. Under the bijection of Theorem 2.1.3, the quasiisogeny class corresponding to the Weil $q$-integer $\pi$ is taken to the quasi-isogeny class corresponding to $\pi^{r}$.

\subsection{Abelian varieties over $\overline{\mathbb{F}}_{p}$}

In this section we state the classification of abelian varieties over $\overline{\mathbb{F}}_{p}$, which is a consequence of the classification over finite fields. We follow the treatment given in [HT01, Sec. V.2].

Suppose that $A$ is a simple abelian variety over $\overline{\mathbb{F}}_{p}, E=\operatorname{End}^{0}(A)$, and $M$ the center of $E$.

Suppose that the prime $p$ splits in $M$ as

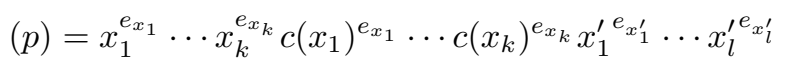

where the primes $x_{i}^{\prime}$ are $c$-invariant. For each prime $x$ of $M$ dividing $p$, let $f_{x}$ denote the residue degree, and define

$$
d_{x}=\left[M_{x}: \mathbb{Q}_{p}\right]
$$


to be the local degree, so that $d_{x}=e_{x} f_{x}$.

The decomposition $M_{p}=\prod_{x \mid p} M_{x}$ induces a decomposition of the $p$-divisible group $A(p)$ (in the quasi-isogeny category)

$$
A(p) \simeq \bigoplus_{x \mid p} A(x) .
$$

There is a corresponding decomposition of the $p$-complete algebra $E_{p}$ into simple local algebras

$$
E_{p}=\prod_{x \mid p} E_{x}
$$

The following theorem appears in [WM71].

Theorem 2.2.1 (Tate). Let $A$ and $A^{\prime}$ be abelian varieties over $\overline{\mathbb{F}}_{p}$. The canonical map

$$
\operatorname{Hom}\left(A, A^{\prime}\right)_{p} \stackrel{\cong}{\longrightarrow} \operatorname{Hom}\left(A(p), A^{\prime}(p)\right)
$$

is an isomorphism.

Therefore, there are isomorphisms

$$
E_{x} \cong \operatorname{End}^{0}(A(x))
$$

Because each of the algebras $E_{x}$ is simple, each of the $p$-divisible groups $A(x)$ must have pure slope $s_{x}$ (or equivalently, be quasi-isogenous to a sum of isomorphic simple $p$-divisible groups of slope $s_{x}$ ). By relabeling the primes, we may assume that $s_{x_{i}} \leq s_{c\left(x_{i}\right)}$. Since $\left[E_{x}: M_{x}\right]=m^{2}, A(x)$ must be of height $m$ as a $p$-divisible $M_{x}$-module, and hence it must have height $d_{x} m$ as as a $p$-divisible group. Express the dimension of the $p$-divisible group $A(x)$ as

$$
\operatorname{dim} A(x)=\eta_{x} f_{x} d_{x} m
$$

for $\eta_{x} \in \mathbb{Q}$.

We analyze the Newton polygons of these $p$-divisible groups. For the primes $x_{i}$, the polarization $\lambda$ induces a quasi-isogeny

$$
\lambda_{*}: A\left(x_{i}\right) \rightarrow A\left(c\left(x_{i}\right)\right)^{\vee} .
$$

Thus we must have

$$
s_{x_{i}}=1-s_{c\left(x_{i}\right)} .
$$

The dimension of $A\left(x_{i}\right) \oplus A\left(c\left(x_{i}\right)\right)$ may be computed:

$$
\begin{aligned}
\operatorname{dim} A\left(x_{i}\right) \oplus A\left(c\left(x_{i}\right)\right) & =s_{x_{i}} d_{x_{i}} m+s_{c\left(x_{i}\right)} d_{c\left(x_{i}\right)} m \\
& =s_{x_{i}} d_{x_{i}} m+\left(1-s_{x_{i}}\right) d_{x_{i}} m \\
& =d_{x_{i}} m .
\end{aligned}
$$

The Newton polygon for $A\left(x_{i}\right) \oplus A\left(c\left(x_{i}\right)\right)$ therefore must take the following form. 


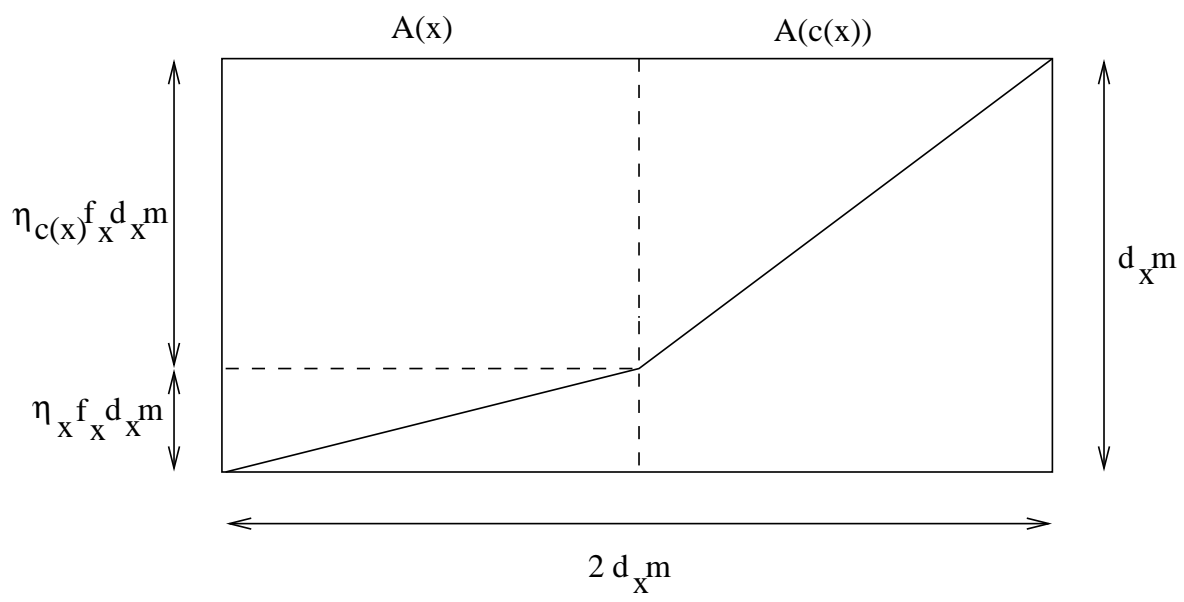

For the primes $x_{j}^{\prime}$, the polarization induces a quasi-isogeny

$$
\lambda: A\left(x_{j}^{\prime}\right) \rightarrow A\left(x_{j}^{\prime}\right)^{\vee} .
$$

This implies that $s_{x_{j}^{\prime}}=1-s_{x_{j}^{\prime}}$, which forces $s_{x_{j}^{\prime}}=1 / 2$. The Newton polygon for $A\left(x_{j}^{\prime}\right)$ therefore takes the following form.

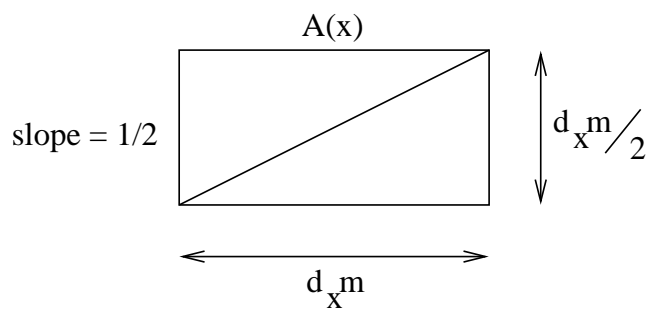

Since the dimension of the $p$-divisible group $A(p)$ must be the dimension of $A$, we deduce that

$$
\operatorname{dim} A=d m / 2 .
$$

We also see that for all $x$, the values $\eta_{x}$ must satisfy

$$
\eta_{x} f_{x}+\eta_{c(x)} f_{x}=1
$$

The slopes are recovered by the formula

$$
s_{x}=\eta_{x} f_{x} .
$$

The data $\left(M,\left(\eta_{x}\right)\right)$ consisting of a CM field $M$ and $\left(\eta_{x}\right)$ satisfying condition (2.2.2) is called a $p$-adic type. The $p$-adic type associated to $A$ is independent of the polarization $\lambda$, and invariant under quasi-isogeny. If $A$ is an abelian variety defined over $\mathbb{F}_{p^{r}}$ with associated CM field $M$ and Frobenius morphism $\pi \in M$, the associated $p$-adic type is $(M,(x(\pi) / r))$. (Here the valuation $x$ is normalized so that $x(p)=e_{x}$.)

We may form a category of $p$-adic types. A morphism

$$
\left(M,\left(\eta_{x}\right)\right) \rightarrow\left(M^{\prime},\left(\eta_{x^{\prime}}^{\prime}\right)\right)
$$


consists of an embedding $M \hookrightarrow M^{\prime}$ such that if $x$ is a prime of $M$, and $x^{\prime}$ is a prime of $M^{\prime}$ dividing $x$, then we have

$$
\eta_{x^{\prime}}^{\prime}=e_{x^{\prime} / x} \eta_{x}
$$

A $p$-adic type $\left(M,\left(\eta_{x}\right)\right)$ is said to be minimal if it is not the target of a non-trivial morphism in the category of all $p$-adic types. The $p$-adic type associated to a simple abelian variety $A$ is minimal.

Theorem 2.2.3 (Honda-Tate).

(1) The natural map

$$
\begin{gathered}
\left\{\text { quasi-isogeny classes of simple abelian varieties over } \overline{\mathbb{F}}_{p}\right\} \\
\downarrow \\
\{\text { minimal } p \text {-adic types }\}
\end{gathered}
$$

is a bijection.

(2) If $A$ is the simple abelian variety associated to a minimal p-adic type $\left(M,\left(\eta_{x}\right)\right)$, then the local invariants of $E=\operatorname{End}^{0}(A)$ are given by

$$
\begin{aligned}
\operatorname{inv}_{x} E & =1 / 2, & & x \text { real, } \\
\operatorname{inv}_{x} E & =0, & & x \nmid p, \\
\operatorname{inv}_{x} E & =\eta_{x} f_{x}, & & x \mid p .
\end{aligned}
$$

For our homotopy theoretical applications, we are interested in the most basic abelian varieties $A$ over $\overline{\mathbb{F}}_{p}$ for which the $p$-divisible group $A(p)$ contains a 1dimensional formal group of height $n$. The Honda-Tate classification tells us that for $n>2$, the smallest such example will have $p$-adic type $\left(M,\left(\eta_{x}\right)\right)$ where

(1) $M$ is a quadratic imaginary extension of $\mathbb{Q}$ in which $p$ splits as $x c(x)$.

(2) $\eta_{x}=1 / n$ and $\eta_{c(x)}=(n-1) / n$.

Furthermore, given any quadratic extension $M$ as above with a chosen prime $x$ over $p$, there exists a unique quasi-isogeny class of abelian variety of dimension $n$ with complex multiplication by $F$ such that $A(x)$ has slope $1 / n$.

Let $B$ be a simple algebra whose center $F$ is a CM field, so that $[B: F]=s^{2}$. We shall want to consider abelian varieties $A$ with the data of a fixed embedding

$$
i: B \hookrightarrow \operatorname{End}^{0}(A) \text {. }
$$

We consider the semisimple category $\operatorname{AbVar}_{\mathbb{F}_{p}}^{0}(B)$ consisting of such pairs $(A, i)$, where the morphisms are $B$-linear quasi-homomorphisms. We shall refer to such objects as $B$-linear abelian varieties.

Kottwitz [Kot92, Sec. 3] makes some very general observations concerning this set-up, which we specialize to our case below, following [HT01, V.2]. A $p$-adic type over $F$ is a $p$-adic type $\left(L,\left(\eta_{x}\right)\right)$ where $L$ is an extension of $F$. A $p$-adic type over $F$ is minimal if it is minimal in the category of $p$-adic types over $F$. Every simple $B$-linear abelian variety $(A, i)$ is isotypical (isogenous to $A_{0}^{j}$ for $A_{0}$ simple) when viewed as an object of $\operatorname{AbVar}_{\mathbb{F}_{p}}^{0}[$ Kot92, Lem. 3.2]. 
Given a $B$-linear abelian variety $(A, i)$ which is isogenous to $A_{0}^{j}$ with $A_{0}$ simple, define the following:

$$
\begin{aligned}
E & =\operatorname{End}^{0}\left(A_{0}\right), \\
M & =\text { center of } E, \\
D & =\operatorname{End}_{B}^{0}(A), \\
L & =\text { center of } D, \\
t & =[D: L]^{1 / 2} .
\end{aligned}
$$

Observe that $M$ is naturally contained in $L$, since it is central in $\operatorname{End}^{0}(A)$. Observe also that $F$, when viewed as lying in $\operatorname{End}^{0}(A)$, also must be contained in $L$. Kottwitz [Kot92, Lem. 3.3] observes that $L$ is a factor of $F \otimes_{\mathbb{Q}} M$. He shows that this gives a correspondence

\{simple $B$-linear abelian varieties $(A, i), A$ isotypical of type $\left.A_{0}\right\}$

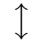

\{fields $L$ occurring as factors of $F \otimes_{\mathbb{Q}} M$ \}

Since $L$ is a factor of $F \otimes_{\mathbb{Q}} M$, and both $F$ and $M$ are CM fields, $L$ must be a CM field.

Let $\left(M,\left(\nu_{y}\right)\right)$ be the $p$-adic type of $A_{0}$. We may associate to $A$ the $p$-adic type $\left(L,\left(\eta_{x}\right)\right)$ over $F$, where, if $x$ is a prime of $L$ lying over a prime $y$ of $M$, the value of $\eta_{x}$ is given by

$$
\eta_{x}=e_{x / y} \nu_{y}
$$

If $A$ is simple in $\operatorname{AbVar}_{\mathbb{F}_{p}}^{0}(B)$, then the associated $p$-adic type $\left(L, \eta_{x}\right)$ is minimal over $F$.

If $x$ is a place of $L$ which is not invariant under the action of the conjugation $c$, then the Newton polygon for the $p$-divisible $B$-module $A(x) \oplus A(c(x))$ is displayed below.

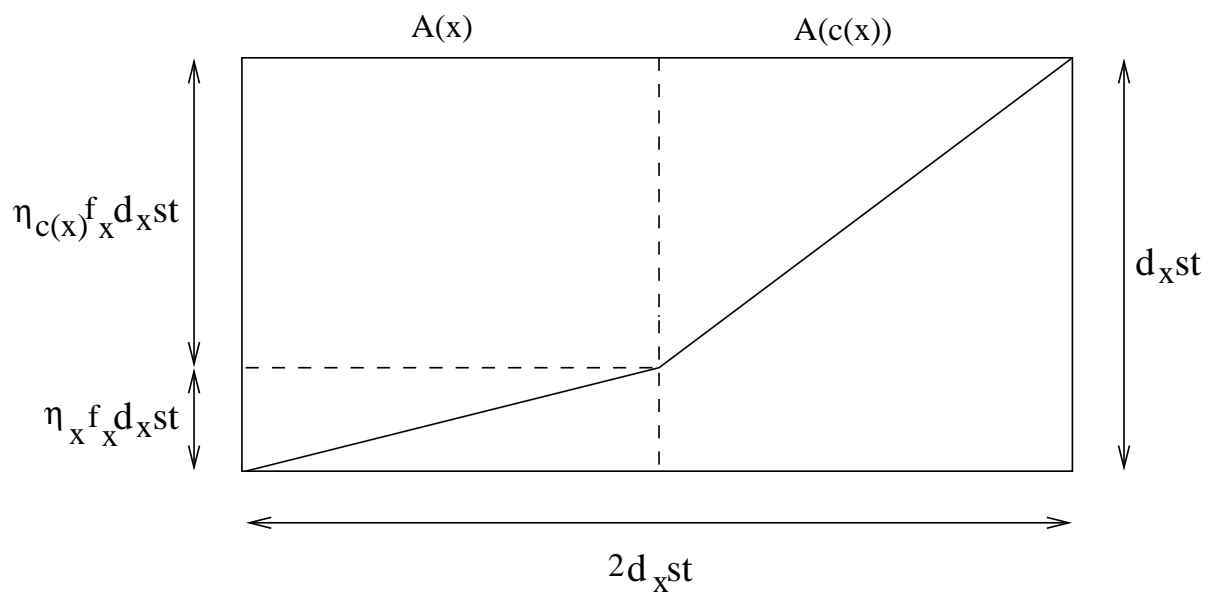

Otherwise, if $x$ is a place of $L$ which is invariant under the conjugation action, then the Newton polygon of the $p$-divisible group $A(x)$ takes the following form. 


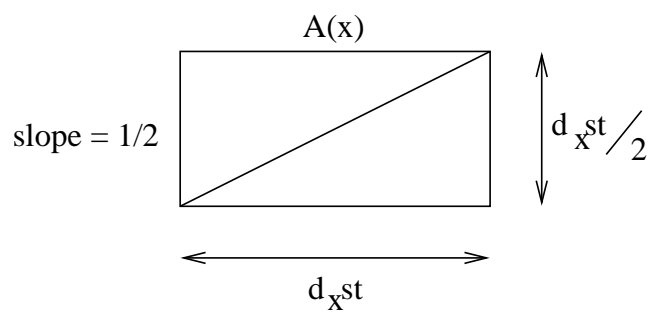

The dimension of the abelian variety $A$ is related to this data by the formula

$$
\operatorname{dim} A=d s t / 2 .
$$

Kottwitz proves the following variation of the Honda-Tate classification for $B$-linear abelian varieties [Kot92, Lem. 10.13].

TheOREM 2.2.4 (Kottwitz). The simple objects of $\operatorname{AbVar}_{\mathbb{F}_{p}}^{0}(B)$ are classified as follows.

(1) The natural map

$\left\{\right.$ quasi-isogeny classes of simple B-linear abelian varieties over $\left.\overline{\mathbb{F}}_{p}\right\}$

$\{$ minimal p-adic types over $F\}$

is a bijection.

(2) If $A$ is the simple B-linear abelian variety associated to a minimal $p$ adic type $\left(L,\left(\eta_{x}\right)\right)$ over $F$, then the local invariants of the central division algebra $D=\operatorname{End}_{B}^{0}(A)$ over $L$ are given by:

$$
\begin{array}{lll}
\operatorname{inv}_{x} D=1 / 2-\operatorname{inv}_{x}\left(B \otimes_{F} L\right), & & x \text { real, } \\
\operatorname{inv}_{x} D=-\operatorname{inv}_{x}\left(B \otimes_{F} L\right), & & x \not p, \\
\operatorname{inv}_{x} D=\eta_{x} f_{x}-\operatorname{inv}_{x}\left(B \otimes_{F} L\right), & & x \mid p .
\end{array}
$$





\section{CHAPTER 3}

\section{Tate modules and level structures}

\subsection{Tate modules of abelian varieties}

Let $A$ be an abelian variety over an algebraically closed field $k$. Assume $\ell$ is a prime distinct from the characteristic of $k$. Let

$$
A\left[\ell^{\infty}\right] \subseteq A(k)
$$

denote the $\ell$-torsion subgroup of $k$-points of $A$. If $A$ is an abelian variety of dimension $g$, then there is an abstract isomorphism

$$
A\left[\ell^{\infty}\right]=\underset{i}{\lim } A\left[\ell^{i}\right] \cong\left(\mathbb{Z} / \ell^{\infty}\right)^{2 g} .
$$

The (covariant) $\ell$-adic Tate module of $A$ is defined as the inverse limit

$$
T_{\ell}(A)=\varliminf_{i} A\left[\ell^{i}\right] \cong \mathbb{Z}_{\ell}^{2 g} .
$$

The inverse limit is taken over the $\ell$ th power maps. We define

$$
V_{\ell}(A)=T_{\ell}(A) \otimes \mathbb{Q} .
$$

Let $\mathbb{Z}_{\ell}(1)$ denote the $\ell$-adic Tate module of the multiplicative group,

$$
\mathbb{Z}_{\ell}(1)={\underset{\leftarrow}{i}}_{\lim _{i}} \mu_{\ell^{i}} \cong \mathbb{Z}_{\ell} .
$$

There are natural isomorphisms

$$
A^{\vee}\left[\ell^{i}\right] \cong \operatorname{Hom}\left(A\left[\ell^{i}\right], \mu_{\ell^{i}}\right),
$$

and taking limits gives rise to a natural isomorphism

$$
T_{\ell}\left(A^{\vee}\right) \cong \operatorname{Hom}_{\mathbb{Z}_{\ell}}\left(T_{\ell}(A), \mathbb{Z}_{\ell}(1)\right) .
$$

It is useful to consider the collection of all Tate modules at once. Let $p$ be the characteristic of $k$. We will denote

$$
T^{p}(A)=\prod_{\ell \neq p} T_{\ell}(A) .
$$

Tensoring with $\mathbb{Q}$, we get a module over $\mathbb{A}^{p, \infty}$ :

$$
V^{p}(A)=T^{p}(A) \otimes \mathbb{Q} .
$$




\subsection{Virtual subgroups and quasi-isogenies}

Let $A$ be an abelian variety over $k$, an algebraically closed field of characteristic $p$.

For a subring $R$ of $\mathbb{Q}$, an $R$-isogeny of abelian varieties $A \rightarrow A^{\prime}$ is a quasi-isogeny that lies in

$$
R \otimes_{\mathbb{Z}} \operatorname{Hom}\left(A, A^{\prime}\right) .
$$

An invertible $R$-isogeny is one whose inverse is also an $R$-isogeny.

Let $A\left[\operatorname{tor}^{p}\right]$ be the subgroup of $A(k)$ consisting of torsion elements of order prime to $p$. There is a natural surjection

$$
\pi_{A}: V^{p}(A) \rightarrow A\left[\text { tor }^{p}\right]
$$

with kernel $T^{p}(A)$. For each finite subgroup $H \subset A\left[\operatorname{tor}^{p}\right]$ there is a corresponding lattice $\pi_{A}^{-1}(H) \subset V^{p}(A)$ such that $T^{p}(A) \subseteq \pi^{-1}(H)$.

More generally, we can define the set of prime-to-p virtual subgroups of $A, \operatorname{VSub}^{p}(A)$, to be the set of lattices $K \subset V^{p}(A)$. For any such $K$, there exists some integer $N$ with $N \cdot T^{p}(A) \subseteq K$ and $\left[K: N \cdot T^{p}(A)\right]<\infty$.

A $\mathbb{Z}_{(p)}$-isogeny $\phi: A \rightarrow A^{\prime}$ induces an isomorphism $V^{p}(A) \rightarrow V^{p}\left(A^{\prime}\right)$. We define the kernel of the quasi-isogeny $\phi$ to be

$$
\operatorname{Ker}(\phi)=\phi^{-1}\left(T^{p}\left(A^{\prime}\right)\right) \in \operatorname{VSub}^{p}(A) .
$$

Given a virtual subgroup $H \subset V^{p}(A)$ there is a way to associate a quasi-isogeny with kernel $H$. Let $N$ be an integer such that $T^{p}(A) \subseteq N^{-1} H$, so the image of $N^{-1} H$ in $A$ is a finite subgroup $K$. The quasi-isogeny $\phi_{H}$ associated to $H$ is the isogeny

$$
\phi_{H}: A \stackrel{\phi_{K}}{\longrightarrow} A / K \stackrel{\left[N^{-1}\right]}{\longrightarrow} A / K
$$

where $\phi_{K}$ is the quotient isogeny associated to $K$, and $\left[N^{-1}\right]$ is the quasi-isogeny which is inverse to the $N$ th power map. The quasi-isogeny $\phi_{H}$ actually depends on the choice of $N$, but a different choice $N^{\prime}$ yields an isogeny which is canonically isomorphic. Indeed, consider the following diagram.

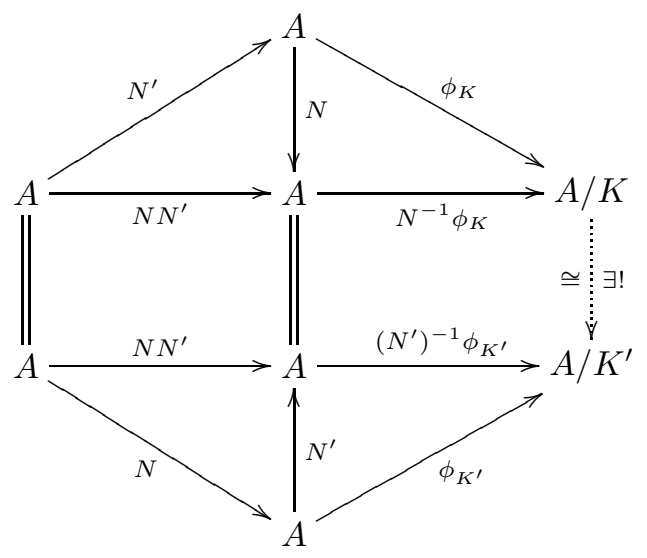


The composites given by the two rows in the above diagram are actual isogenies, and the canonical isomorphism in the diagram above exists because the kernels of these isogenies are equal. For now we will commit the of abuse of defining $A / H$ to be $A / K$.

\subsection{Level structures}

Let $V$ be a rational vector space of dimension $2 n$, and fix a $\mathbb{Z}$-lattice $L \subset V$. Define

$$
\begin{aligned}
L^{p} & =\prod_{\ell \neq p} L \otimes \mathbb{Z}_{\ell}, \\
V^{p} & =V \otimes \mathbb{Q} \mathbb{A}^{p, \infty}=L^{p} \otimes \mathbb{Q} .
\end{aligned}
$$

Assume that $A$ is an abelian variety of dimension $n$ over an algebraically closed field $k$ of characteristic $p$. By an integral uniformization we shall mean an isomorphism

$$
\eta: L^{p} \stackrel{\cong}{\longrightarrow} T^{p}(A) .
$$

By a rational uniformization we shall mean an isomorphism

$$
\eta: V^{p} \stackrel{\cong}{\longrightarrow} V^{p}(A)
$$

Let $K_{0}^{p}=\operatorname{Aut}\left(L^{p}\right)$ be subgroup of $\operatorname{Aut}\left(V^{p}\right)$ given by the lattice automorphisms:

$$
K_{0}^{p}=\left\{g \in \operatorname{Aut}\left(V^{p}\right): g\left(L^{p}\right)=L^{p}\right\} .
$$

There is a natural action of $\operatorname{Aut}\left(V^{p}\right)$ on the set of rational uniformizations of $A$. Let $K^{p}$ be a subgroup of Aut $\left(V^{p}\right)$. A rational $K^{p}$-level structure $[\eta]_{K^{p}}$ is the $K^{p}$-orbit of a rational uniformization $\eta$. We say that $[\eta]_{K^{p}}$ is an integral $K^{p}$-level structure if $\eta$ is an integral uniformization.

Let $\mathcal{L}_{K_{0}^{p}}(A)$ be the set of rational $K_{0}^{p}$-level structures on $A$. There is a natural map

$$
\kappa: \mathcal{L}_{K_{0}^{p}}(A) \rightarrow \operatorname{VSub}^{p}(A) .
$$

Given $[\eta] \in \mathcal{L}_{K_{0}^{p}}(A)$, define

$$
\kappa([\eta])=\eta\left(L^{p}\right) .
$$

Indeed, there exists a positive integer $N$ such that $\eta\left(L^{p}\right)$ contains $N \cdot T^{p}(A)$. Since the image $\eta\left(L^{p}\right)$ is compact, the index $\left[\eta\left(L^{p}\right): N \cdot T^{p}(A)\right]$ must be finite. Therefore $\eta\left(L^{p}\right)$ is a virtual subgroup $\kappa(\eta) \in \mathrm{VSub}^{p}(A)$. Automorphisms of $L^{p}$ do not alter the image $\eta\left(L^{p}\right)$, so the virtual subgroup $\eta\left(L^{p}\right)$ depends only on the $K_{0}^{p}$-orbit of $\eta$.

Lemma 3.3.1. A rational level structure $\eta: V^{p} \cong V^{p}(A)$ restricts to an integral level structure if and only if the virtual subgroup $\kappa(\eta)$ is $T^{p}(A)$. 


\subsection{The Tate representation}

Let $k$ be a field, and let $\ell$ be distinct from the characteristic of $k$. If $A$ is an abelian variety over $k$, the $\ell$-adic Tate module of $A$ is defined by

$$
T_{\ell}(A)=T_{\ell}\left(A \otimes_{k} \bar{k}\right) \text {. }
$$

The Tate module gives a faithful representation of the category of abelian varieties over $k$ (See, for instance, [Mil98, Lem. 9.6]).

Proposition 3.4.1. Let $A$ and $A^{\prime}$ be abelian varieties over $k$. The natural map

$$
\operatorname{Hom}\left(A, A^{\prime}\right) \otimes \mathbb{Z}_{\ell} \rightarrow \operatorname{Hom}_{\mathbb{Z}_{\ell}}\left(T_{\ell} A, T_{\ell} A^{\prime}\right)
$$

is a monomorphism.

The integral Tate module can be used to isolate the lattice of homomorphisms using the following lemma.

Lemma 3.4.2. Let $A$ and $A^{\prime}$ be abelian varieties over $k$. The diagram

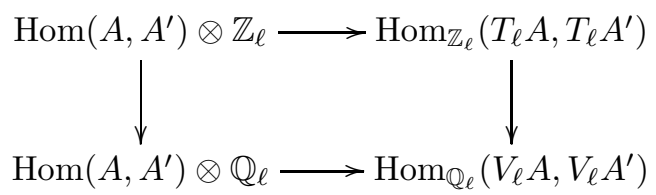

is a pullback square.

Proof. Suppose that $f \in \operatorname{Hom}\left(A, A^{\prime}\right) \otimes \mathbb{Q}_{\ell}$ has the property that $f_{*}\left(T_{\ell} A\right) \subseteq$ $T_{\ell} A^{\prime}$. There is some $i$ so that $g=\ell^{i} f$ is contained in $\operatorname{Hom}\left(A, A^{\prime}\right) \otimes \mathbb{Z}_{\ell}$. Then $g_{*}\left(T_{\ell} A\right)$ is contained in $\ell^{i} T_{\ell} A^{\prime}$. Express $g$ in the form $g=g_{1}+\ell^{i} g_{2}$ for $g_{1} \in \operatorname{Hom}\left(A, A^{\prime}\right)$ and $g_{2} \in \operatorname{Hom}\left(A, A^{\prime}\right) \otimes \mathbb{Z}_{\ell}$. We deduce that the map

$$
\left(g_{1}\right)_{*}: A\left[\ell^{i}\right] \rightarrow A^{\prime}\left[\ell^{i}\right]
$$

is null. We therefore have the following factorization.

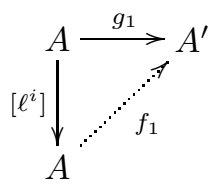

We deduce that $f=f_{1}+g_{2}$ lies in $\operatorname{Hom}\left(A, A^{\prime}\right) \otimes \mathbb{Z}_{\ell}$.

\subsection{Homomorphisms of abelian schemes}

In this section we explain how the results on abelian varieties given in the last section extend to abelian schemes. Fix a connected base scheme $S$, let $x$ be a point of $S$, and let $A$ and $A^{\prime}$ be abelian schemes over $S$. As observed in [Fis75], the following proposition follows immediately from [Mum65, Cor 6.2].

Proposition 3.5.1. The restriction map

$$
\operatorname{Hom}_{S}\left(A, A^{\prime}\right) \rightarrow \operatorname{Hom}_{x}\left(A_{x}, A_{x}^{\prime}\right)
$$

is a monomorphism. 
Lemma 3.5.2. Suppose that $S$ is a scheme over $\mathbb{Z}[1 / \ell]$. Then the diagram

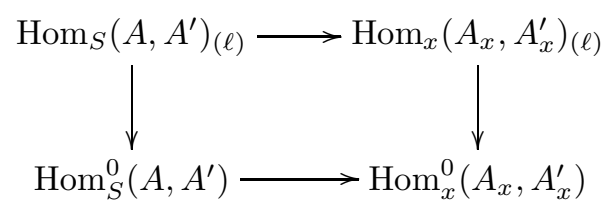

is a pullback.

Proof. Suppose that $f \in \operatorname{Hom}_{S}^{0}\left(A, A^{\prime}\right)$ has the property that $f_{x}$ actually lies in $\operatorname{Hom}_{x}\left(A_{x}, A_{x}^{\prime}\right)_{(\ell)}$. Let $N$ coprime to $\ell$ and $i$ be chosen so that $g=\ell^{i} N f$ lies in $\operatorname{Hom}_{S}\left(A, A^{\prime}\right)$. Then the homomorphism of étale group schemes

$$
g_{x}: A_{x}\left[\ell^{i}\right] \rightarrow A_{x}^{\prime}\left[\ell^{i}\right]
$$

over $x$ is null. Consider the map

$$
g: A\left[\ell^{i}\right] \rightarrow A^{\prime}\left[\ell^{i}\right]
$$

of étale group schemes over $S$. Now every connected component of $A\left[\ell^{i}\right]$ contains a point of $A_{x}\left[\ell^{i}\right]$, and the zero-section $0_{A^{\prime}}$ is a connected component of $A^{\prime}\left[\ell^{i}\right]$. Since $g$ maps every point of $A_{x}\left[\ell^{i}\right]$ to the component $0_{A^{\prime}}$, we deduce that $g$ must map all of $A\left[\ell^{i}\right]$ to zero. Therefore, we get a factorization

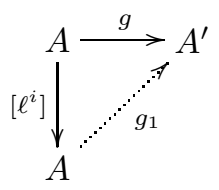

and we see that $f=N^{-1} g_{1}$ lies in $\operatorname{Hom}_{S}\left(A, A^{\prime}\right)_{(\ell)}$.

Lemma 3.5.2 combines with Lemma 3.4.2 to give the following corollary.

Corollary 3.5.3. Let $s$ be a geometric point of $S$. The square

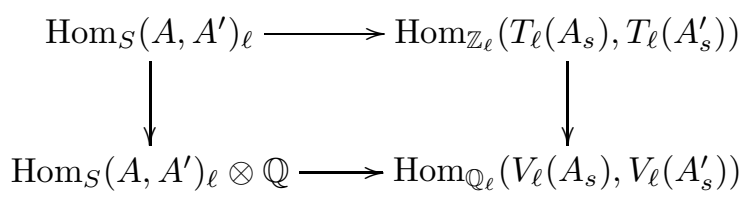

is a pullback. 



\section{CHAPTER 4}

\section{Polarizations}

For us, polarizations serve two purposes. Firstly, the automorphism group of an abelian variety is often infinite. However, the subgroup of automorphisms which preserves a polarization is finite. Therefore, the moduli of polarized abelian varieties is more tractable. Mumford [Mum65] made use of geometric invariant theory to prove representability of moduli spaces of polarized abelian varieties, which serves as a starting point for the representability of the moduli problem associated to PEL Shimura varieties. Secondly, in Chapter 7 we shall see that the structure of a polarization on an abelian variety $A$ can be used to control the deformation theory of a compatible splitting of the $p$-divisible group $A(p)$.

In this chapter we outline some of the theory of polarizations of an abelian variety. We recall the definition of a polarization and the associated Rosati involution. These give rise to Weil pairings on the Tate module. We then describe the classification of polarized abelian varieties over finite fields. Our treatment is based on those of [Hid04], [HT01], and [Kot92].

\subsection{Polarizations}

Let $A$ be an abelian variety defined over a field $k$. The functor

$$
\mathrm{Pic}_{A / k}: k-\text { Schemes } \rightarrow \text { Abelian groups, }
$$

which associates to a $k$-scheme

$$
f: S \rightarrow \operatorname{Spec}(k)
$$

the set

$$
\operatorname{Pic}_{A / k}(S)=\left\{\begin{array}{l}
\text { Isomorphism classes of invertible sheaves over } f^{*} A \text { which } \\
\text { restrict to the trivial line bundle on the subscheme } 0_{f^{*} A}
\end{array}\right\},
$$

is representable by a group scheme $\operatorname{Pic}_{A / k}$ [Mum70, Sec 13]. The dual abelian variety is defined to be the identity component of this scheme:

$$
A^{\vee}=\operatorname{Pic}_{A / k}^{0} .
$$

If $B$ is an abelian variety over $k$, then specifying a $k$-morphism

$$
\alpha: A \rightarrow B^{\vee}
$$

is the same as giving an isomorphism class of line bundles $L_{\alpha}$ over $A \times_{k} B$ which restricts to the trivial line bundle on the subscheme $A \times_{k} 0_{B}$. The morphism $\alpha$ satisfies $\alpha\left(0_{A}\right)=0_{B} \vee$ if and only if the line bundle $L_{\alpha}$ is also trivial when restricted to the subscheme $0_{A} \times{ }_{k} B$. 
Proposition 4.1.1. The map

$$
\operatorname{Hom}\left(A, B^{\vee}\right) \rightarrow\left\{\begin{array}{l}
\text { Isomorphism classes of line bundles on } \\
A \times_{k} B \text {, trivial on } 0_{A} \times_{k} B \text { and } A \times_{k} 0_{A}
\end{array}\right\}
$$

given by sending a homomorphism $\alpha$ to the corresponding line bundle $L_{\alpha}$ is an isomorphism.

Proof. We only need to check that every morphism $\alpha: A \rightarrow B^{\vee}$ such that $\alpha\left(0_{A}\right)=0_{B^{\vee}}$ is a homomorphism. That is, we need the following diagram to commute.

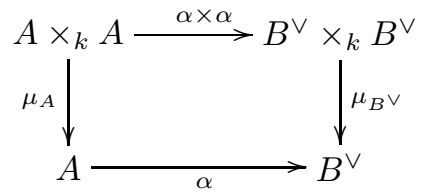

This is equivalent to verifying that the line bundles

$$
L_{\alpha \circ \mu_{A}}=\left(\mu_{A} \times 1\right)^{*} L_{\alpha} \quad \text { and } \quad L_{\mu_{B} \vee \alpha \times \alpha}=p_{1}^{*} L_{\alpha} \otimes p_{2}^{*} L_{\alpha}
$$

over $A \times_{k} A \times_{k} B$ are isomorphic. Here

$$
p_{i}: A \times_{k} A \times_{k} B \rightarrow A \times_{k} B
$$

are the two projections. However, the two line bundles are easily seen to agree on the subschemes $0_{A} \times_{k} A \times_{k} B, A \times_{k} 0_{A} \times_{k} B$, and $A \times_{k} A \times_{k} 0_{B}$. The theorem of the cube $[\mathbf{M i l 9 8}]$ therefore implies that these line bundles must be isomorphic over $A \times_{k} A \times_{k} B$.

The identity map 1: $A^{\vee} \rightarrow A^{\vee}$ gives rise to the Poincaré bundle $L_{1}$ on $A^{\vee} \times_{k} A$. The line bundle $L_{1}$, when viewed as a bundle over $A \times_{k} A^{\vee}$, corresponds to a canonical isomorphism

$$
A \stackrel{\cong}{\longrightarrow}\left(A^{\vee}\right)^{\vee}
$$

Given a homomorphism $\alpha: A \rightarrow B$, there is a dual homomorphism

$$
\alpha^{\vee}: B^{\vee} \rightarrow A^{\vee} \text {. }
$$

(1) Pullback along $\alpha$ gives a natural transformation of functors

$$
\alpha^{*}: \operatorname{Pic}_{B / k} \rightarrow \operatorname{Pic}_{A / k},
$$

hence a map on representing objects, which restricts to $\alpha^{\vee}$ on connected components.

(2) Use the canonical isomorphism $B \cong\left(B^{\vee}\right)^{\vee}$, and take the corresponding line bundle $L_{\alpha}$ over $A \times_{k} B^{\vee}$. The pullback $\tau^{*} L_{\alpha}$ under the twist map

$$
\tau: B^{\vee} \times_{k} A \stackrel{\cong}{\rightrightarrows} A \times_{k} B^{\vee}
$$

corresponds to the homomorphism

$$
\alpha^{\vee}: B^{\vee} \rightarrow A^{\vee} .
$$


A homomorphism $\lambda: A \rightarrow A^{\vee}$ is symmetric if the dual homomorphism

$$
\lambda^{\vee}: A \cong\left(A^{\vee}\right)^{\vee} \rightarrow A^{\vee}
$$

equals $\lambda$. By the second description of the dual homomorphism given above, this condition is equivalent to requiring the line bundle $L_{\lambda}$ over $A \times_{k} A$ to satisfy $L_{\lambda} \cong$ $\tau^{*} L_{\lambda}$, where $\tau: A \times_{k} A \rightarrow A \times_{k} A$ is the interchange map.

ExAmple 4.1.2. Let $L$ be a line bundle over $A$. Then the line bundle $\mu_{A}^{*} L \otimes$ $p_{1}^{*} L^{-1} \otimes p_{2}^{*} L^{-1}$ is a degree 0 line bundle over $A \times A$ which is trivial on $0_{A} \times{ }_{k} A$ and $A \times_{k} 0_{A}$. Here $\mu_{A}: A \times_{k} A \rightarrow A$ is the multiplication map, and $p_{i}: A \times_{k} A \rightarrow A$ are the two projections. The corresponding homomorphism

$$
\lambda_{L}: A \rightarrow A^{\vee}
$$

is symmetric.

In fact, Theorem 2 in [Mum70, Sec 20], and the remarks which follow it, give the following converse.

THEOREM 4.1.3. If $k$ is algebraically closed, and $\alpha: A \rightarrow A^{\vee}$ is a symmetric homomorphism, then there exists a line bundle $L$ over $A$ so that $\alpha=\lambda_{L}$. The line bundle $L$ satisfies

$$
L^{\otimes 2}=\Delta^{*} L_{\alpha}
$$

where $\Delta: A \rightarrow A \times_{k} A$ is the diagonal.

The following theorem [Mum70, Sec 16] gives a criterion for determining if a symmetric homomorphism $A \rightarrow A^{\vee}$ is an isogeny (has finite kernel).

TheOrem 4.1.4. Suppose that $L$ is a line bundle on $A$. Then the following conditions are equivalent.

(1) The symmetric homomorphism $\lambda_{L}$ is an isogeny.

(2) There is a unique $0 \leq i \leq \operatorname{dim} A$ such that $H_{z a r}^{i}(A, L)$ is non-trivial, and $H_{z a r}^{j}(A, L)=0$ for $j \neq i$.

The integer $i=i(L)$ in the previous theorem is called the index of $L$. The index is 0 if $L$ is ample [Har77, III.5.3].

Definition 4.1.5. Let $A$ be an abelian variety defined over an algebraically closed field $k$. A polarization of $A$ is a symmetric isogeny

$$
\lambda=\lambda_{L}: A \rightarrow A^{\vee}
$$

determined by a line bundle $L$ which is ample.

REMARK 4.1.6. Abelian varieties are in particular projective, and hence admit ample line bundles. Therefore, every abelian variety admits a polarization.

REMARK 4.1.7. The theory of symmetric homomorphisms for abelian varieties is analogous to the theory of symmetric bilinear forms for real vector spaces. Let $B(-,-)$ be a symmetric bilinear form on a real vector space $V$. The symmetric homomorphism $\lambda_{L}$ associated to a line bundle $L$ is analogous to the symmetric bilinear form

$$
B(x, y)=Q(x+y)-Q(x)-Q(y)
$$


associated to a quadratic form $Q$. Theorem 4.1.3 is an analog to the fact that every form $B$ arises this way, with

$$
Q(x)=\frac{1}{2} B(x, x) .
$$

Representing a form $B$ by a hermitian matrix $A$, the index of a symmetric isogeny is analogous to the number of negative eigenvalues of $A$. A polarization is therefore analogous to a positive definite quadratic form.

If $\lambda=\lambda_{L}$ is a polarization, then $n \lambda=\lambda_{L} \otimes n$ is a polarization for $n$ a positive integer. Two polarizations $\lambda$ and $\lambda^{\prime}$ are equivalent if $n \lambda=m \lambda^{\prime}$ for some positive integers $n$ and $m$. A weak polarization is an equivalence class of polarizations.

We defined polarizations only for abelian varieties defined over an algebraically closed field. If $A$ is an abelian scheme over a scheme $S$, then a polarization of $A$ is an isogeny

$$
\lambda: A \rightarrow A^{\vee}
$$

which restricts to a polarization on geometric fibers.

\subsection{The Rosati involution}

Let $A$ be an abelian variety over an algebraically closed field $k$, and let $\lambda=\lambda_{L}$ be the polarization of $A$ associated to an ample line bundle $L$.

The polarization $\lambda$ induces an involution $\dagger$ on the semi-simple $\mathbb{Q}$-algebra $\operatorname{End}^{0}(A)$; for $f \in \operatorname{End}^{0}(A)$, the quasi-endomorphism $f^{\dagger}$ is given by the composite

$$
f^{\dagger}: A \stackrel{\lambda}{\longrightarrow} A^{\vee} \stackrel{f^{\vee}}{\longrightarrow} A^{\vee} \stackrel{\lambda^{-1}}{\longrightarrow} A .
$$

This involution is the Rosati involution associated to the polarization $\lambda$. If the polarization $\lambda$ is replaced by $n \lambda$ for some $n>0$, the associated Rosati involution remains the same. Therefore, the Rosati involution depends only on the weak polarization determined by $\lambda$.

The positivity of the line bundle $L$ implies that the Rosati involution is positive in the following sense.

Theorem 4.2.1 ([Mum70, Sec 21]). Let $E=\operatorname{End}^{0}(A)$, and suppose $f \in E$ is nonzero. Then

$$
\operatorname{Tr}_{E / \mathbb{Q}}\left(f f^{\dagger}\right)>0
$$

Here $\operatorname{Tr}_{E / \mathbb{Q}}$ is the trace map.

\subsection{The Weil pairing}

Suppose that $k$ is algebraically closed, and that $\lambda: A \rightarrow A^{\vee}$ is a polarization of $A$. Then $\lambda$ induces a map

$$
\lambda_{*}: A\left[\ell^{i}\right] \rightarrow A^{\vee}\left[\ell^{i}\right] \cong \operatorname{Hom}\left(A\left[\ell^{i}\right], \mu_{\ell^{i}}\right)
$$

whose adjoint gives the $\lambda$-Weil pairing

$$
\lambda\langle-,-\rangle_{\ell^{i}}: A\left[\ell^{i}\right] \times A\left[\ell^{i}\right] \rightarrow \mu_{\ell^{i}} .
$$


Taking the inverse limit over $i$, we recover a natural bilinear pairing

$$
\lambda\langle-,-\rangle: T_{\ell}(A) \times T_{\ell}(A) \rightarrow \mathbb{Z}_{\ell}(1)
$$

called the $\lambda$-Weil pairing. The induced pairing

$$
\lambda\langle-,-\rangle: V_{\ell}(A) \otimes_{\mathbb{Q}_{\ell}} V_{\ell}(A) \rightarrow \mathbb{Q}_{\ell}(1)
$$

is non-degenerate, because $\lambda$ is an isogeny. It can be shown that the pairing $\lambda\langle-,-\rangle$ is alternating [Mum70, Sec 20].

\subsection{Polarizations of $B$-linear abelian varieties}

Let $A$ be an abelian variety over a field $k$. Let $B$ be a simple $\mathbb{Q}$-algebra. Recall from Section 2.2 that a $B$-linear abelian variety $(A, i)$ is an abelian variety $A / k$ together with an embedding of rings $i: B \hookrightarrow \operatorname{End}^{0}(A)$. Suppose that $*$ is an involution on $B$.

Definition 4.4.1. A polarization $\lambda$ on $(A, i)$ is compatible if the $\lambda$-Rosati involution restricts to the involution $*$ on $B$.

Theorem 4.2.1 implies that for a compatible polarization to exist, the involution * must be positive. Conversely, we have the following lemma.

Lemma 4.4.2 ([Kot92, Lemma 9.2]). If $*$ is a positive involution on $B$, and $(A, i)$ is a B-linear abelian variety, then there exists a compatible polarization $\lambda$ on $A$.

The $B$-linear structure on $A$ gives the Tate module $V_{\ell}(A)$ the structure of a $B$ module. The compatibility condition on the polarization implies that the $\lambda$-Weil pairing is $*$-hermitian: for all $x, y \in V_{\ell}(A)$ and $b \in B$, we have

$$
\lambda\langle b x, y\rangle=\lambda\left\langle x, b^{*} y\right\rangle .
$$

\subsection{Induced polarizations}

Let $\lambda=\lambda_{L}$ be a polarization of an abelian variety $A$, with associated ample line bundle $L$. Let

$$
\alpha: A^{\prime} \rightarrow A
$$

be an isogeny of abelian varieties. Then $\alpha$ induces an isogeny $\alpha^{*} \lambda$ by the composite

$$
\alpha^{*} \lambda: A^{\prime} \stackrel{\alpha}{\longrightarrow} A \stackrel{\lambda}{\rightarrow} A^{\vee} \stackrel{\alpha^{\vee}}{\longrightarrow}\left(A^{\prime}\right)^{\vee} .
$$

It is easily verified that $\alpha^{*} \lambda$ is the symmetric homomorphism associated to the line bundle $\alpha^{*} L$. Since this line bundle is ample, we see that $\alpha^{*} \lambda$ is a polarization of $A^{\prime}$.

Suppose that $(A, \lambda)$ and $\left(A^{\prime}, \lambda^{\prime}\right)$ are polarized abelian varieties. An isogeny

$$
\alpha: A^{\prime} \rightarrow A
$$

is an isometry if $\alpha^{*} \lambda=\lambda^{\prime}$. If $\alpha^{*} \lambda$ is only equivalent to $\lambda^{\prime}$, we say that $\alpha$ is a similitude. The following lemma follows immediately from the definition of the Rosati involution. 
Lemma 4.5.1. Let $A$ be an abelian variety with polarization $\lambda$ and let $\dagger$ be the Rosati involution on $\operatorname{End}^{0}(A)$. Let $\alpha: A \rightarrow A$ be an isogeny. Then:

$$
\begin{aligned}
& \alpha \text { is an isometry } \Leftrightarrow \alpha^{\dagger} \alpha=1, \\
& \alpha \text { is a similitude } \Leftrightarrow \alpha^{\dagger} \alpha \in \mathbb{Q}^{\times} .
\end{aligned}
$$

An isometry $\alpha: A \rightarrow A$ of a polarized abelian variety $(A, \lambda)$ induces an isometry

$$
\alpha_{*}:\left(V_{\ell}(A), \lambda\langle-,-\rangle\right) \rightarrow\left(V_{\ell}(A), \lambda\langle-,-\rangle\right)
$$

of the $\lambda$-Weil pairing on each $\ell$-adic Tate module. If $\alpha$ is merely a similitude, then $\alpha_{*}$ is a similitude of the $\lambda$-Weil pairing: we have

$$
\lambda\left\langle\alpha_{*}(x), \alpha_{*}(y)\right\rangle=\nu(\alpha) \lambda\langle x, y\rangle
$$

for some $\nu(\alpha) \in \mathbb{Q}^{\times}$, and all $x, y \in V_{\ell}(A)$.

\subsection{Classification of weak polarizations}

Assume that $k$ has finite characteristic $p$. We briefly recall the classification up to isogeny of compatible weak polarizations on a $B$-linear abelian variety $(A, i)$, following [HT01, V.3].

Consider the following:

$$
\begin{aligned}
B & =\text { simple } \mathbb{Q} \text {-algebra, } \\
* & =\text { positive involution on } B \\
(A, i) & =B \text {-linear abelian variety over an algebraically closed field } k, \\
\lambda & =\text { compatible polarization of }(A, i) .
\end{aligned}
$$

Define an algebraic group $H_{(A, i, \lambda)} / \mathbb{Q}$ by

$$
H_{(A, i, \lambda)}(R)=\left\{h \in\left(\operatorname{End}_{B}^{0}(A) \otimes R\right)^{\times}: h^{\dagger} h \in R^{\times}\right\} .
$$

Here, $\dagger$ is the $\lambda$-Rosati involution. Note that by Lemma 4.5.1, $H_{(A, i, \lambda)}(\mathbb{Q})$ is the group of quasi-endomorphisms of $A$ which are similitudes of the polarization $\lambda$.

We shall say that a compatible polarization $\lambda^{\prime}$ is lies in the same similitude class as $\lambda$ if there exists a quasi-isometry $\alpha: A \rightarrow A$ so that $\alpha^{*} \lambda$ is equivalent to $\lambda$.

Lemma 4.6.1 ([HT01, Lem V.3.1]). There is a bijective correspondence between similitude classes of compatible weak polarizations on $A$ and elements in the Galois cohomology kernel:

$$
\operatorname{ker}\left(H^{1}\left(\mathbb{Q}, H_{(A, i, \lambda)}\right) \rightarrow H^{1}\left(\mathbb{R}, H_{(A, i, \lambda)}\right)\right)
$$

REmark 4.6.2. Non-abelian Galois $H^{1}$ is a pointed set. The kernel is the collection of elements which restrict to the distinguished element at the completion $\infty$ of $\mathbb{Q}$. This condition reflects the fact that the index of the symmetric homomorphism $\lambda^{\prime}$ must agree with the index of $\lambda$ in order to be a polarization. 
Assume now that $\ell$ is a prime different from $p$. Let $G U_{V_{\ell}(A)}$ be the algebraic group (over $\mathbb{Q}_{\ell}$ ) of $B$-linear similitudes of $\left(V_{\ell}(A), \lambda\langle-,-\rangle\right.$ ):

$G U_{V_{\ell}(A)}(R)=\left\{g \in\left(\operatorname{End}_{B}\left(V_{\ell}(A)\right) \otimes_{\mathbb{Q}_{\ell}} R\right)^{\times}: \begin{array}{l}\text { there exists } \nu(g) \in R^{\times} \text {such } \\ \text { that } \lambda\langle g(x), g(y)\rangle=\nu(g) \lambda\langle x, y\rangle\end{array}\right\}$.

Similitude classes of non-degenerate $*$-hermitian alternating forms on $V_{\ell}(A)$ are classified by elements of $H^{1}\left(\mathbb{Q}_{\ell}, G U_{V_{\ell}(A)}\right)$.

Since similitudes of $(A, \lambda)$ induce similitudes of $\left(V_{\ell}(A), \lambda\langle-,-\rangle\right)$, there is a homomorphism of algebraic groups

$$
H_{(A, i, \lambda)} \times_{\operatorname{Spec}(\mathbb{Q})} \operatorname{Spec}\left(\mathbb{Q}_{\ell}\right) \rightarrow G U_{V_{\ell}(A)}
$$

which induces a map on Galois cohomology

$$
H^{1}\left(\mathbb{Q}, H_{(A, i, \lambda)}\right) \rightarrow H^{1}\left(\mathbb{Q}_{\ell}, G U_{V_{\ell}}\right) .
$$

Lemma 4.6.3 ([HT01, Lem V.3.1]). Let $\lambda^{\prime}$ be a compatible polarization of $(A, i)$. The image of the cohomology class $\left[\lambda^{\prime}\right] \in H^{1}\left(\mathbb{Q}, H_{(A, i, \lambda)}\right)$ in $H^{1}\left(\mathbb{Q}_{\ell}, G U_{V_{\ell}}(A)\right)$ quantifies the difference between the similitude classes of alternating forms represented by $\lambda^{\prime}\langle-,-\rangle$ and $\lambda\langle-,-\rangle$ on $V_{\ell}(A)$.

In practice, the cohomology class $\left[\lambda^{\prime}\right]$ is often computed by means of its local invariants in this manner. 



\section{CHAPTER 5}

\section{Forms and involutions}

In Section 4.6, we saw that polarized $B$-linear abelian varieties up to isogeny were classified by the Galois cohomology of the similitude group of an alternating form. In this chapter we will explicitly outline the Galois cohomology computations relevant for this book. Most of the results in this chapter may be found elsewhere (see, for instance, [Sch85]), but for convenience we enumerate these results in one place.

\subsection{Hermitian forms}

We shall use the following notation.

$$
\begin{aligned}
F & =\text { quadratic imaginary extension of } \mathbb{Q} . \\
c & =\text { conjugation on } F . \\
B & =\text { central simple algebra over } F \text { of dimension } n^{2} . \\
* & =\text { positive involution on } B \text { of the second kind. } \\
V & =\text { free left } B \text {-module of rank } 1 . \\
C & \left.=\operatorname{End}_{B}(V) \quad \text { (noncanonically isomorphic to } B^{o p}\right) .
\end{aligned}
$$

(An involution of $B$ is said to be of the second kind if it restricts to the conjugation $c$ on $F$.)

REMARK 5.1.1. For $B$ to admit an involution $*$ of the second kind it is necessary and sufficient $[\mathbf{S c h} 85]$ that

$$
\operatorname{inv}_{x} B=0
$$

for all finite primes $x$ which are not split in $F$ and

$$
\operatorname{inv}_{y} B+\operatorname{inv}_{y^{c}} B=0
$$

for all finite primes $x$ which split as $y y^{c}$ in $F$.

In Section 4.6 we explained how classification of compatible weak polarizations on a $B$-linear abelian variety was equivalent to the classification of certain alternating forms. For this reason, we are interested in non-degenerate alternating forms

$$
\langle-,-\rangle: V \otimes_{\mathbb{Q}} V \rightarrow \mathbb{Q}
$$

which are $*$-hermitian, meaning that

$$
\langle b v, w\rangle=\left\langle v, b^{*} w\right\rangle .
$$


for all $b \in B$ and $v, w \in V$. Two $*$-hermitian alternating forms $\langle-,-\rangle$ and $\langle-,-\rangle^{\prime}$ on $V$ are said to be similar if there exists an endomorphism $\alpha \in C$ and a unit $\nu(\alpha) \in \mathbb{Q}^{\times}$so that

$$
\langle\alpha v, \alpha w\rangle^{\prime}=\nu(\alpha)\langle v, w\rangle
$$

for all $v, w \in V$. If $\alpha$ may be chosen so that $\nu(\alpha)=1$, then we shall say that $\langle-,-\rangle$ and $\langle-,-\rangle^{\prime}$ are isometric.

Such an alternating form induces an involution $\iota$ on $C$ via the formula

$$
\langle c v, w\rangle=\left\langle v, c^{\iota} w\right\rangle .
$$

Two similar forms induce the same involution $\iota$.

We shall say that a bilinear form

$$
(-,-): V \otimes_{\mathbb{Q}} V \rightarrow F
$$

is *-symmetric if it is $F$-linear in the first variable, and

$$
(v, w)=(w, v)^{c} .
$$

For definiteness, we shall let

$$
\begin{aligned}
& V=B, \\
& F=\mathbb{Q}(\delta),
\end{aligned}
$$

where $B$ is regarded as a left $B$-module, and $\delta^{2}=d$ for a negative square-free integer $d$. There is a natural identification $C=B^{o p}$.

Lemma 5.1.2. There is an one to one correspondence:

$$
\begin{gathered}
\{\text { non-degenerate } * \text {-hermitian alternating forms }\langle-,-\rangle \text { on } V\} \\
\uparrow \\
\{\text { non-degenerate } * \text {-hermitian } * \text {-symmetric forms }(-,-) \text { on } V\}
\end{gathered}
$$

Proof. Given an alternating form $\langle-,-\rangle$, the corresponding $*$-symmetric form is given by

$$
(v, w)=\langle\delta v, w\rangle+\delta\langle v, w\rangle .
$$

Given a $*$-symmetric form $(-,-)$, the corresponding alternating form is given by

$$
\langle v, w\rangle=\frac{1}{2 d} \operatorname{Tr}_{F / \mathbb{Q}} \delta(v, w)
$$

The non-degeneracy of the involution $*$ allows one to deduce the following lemma, which makes everything more explicit.

Lemma 5.1.3. Let $\langle-,-\rangle$ be a non-degenerate $*$-hermitian alternating form. There exists a unique element $\beta \in B=V$ satisfying $\beta^{*}=-\beta$ which encodes $\langle-,-\rangle$ :

$$
\langle x, y\rangle=\operatorname{Tr}_{F / \mathbb{Q}} \operatorname{Tr}_{B / F}\left(x \beta y^{*}\right) .
$$


Regarding $\beta$ as an element $\gamma$ of $C=B^{o p}$, we have that the involution on $C$ is given by

$$
c^{\iota}=\gamma^{-1} c^{*} \gamma
$$

COROllary 5.1.4. There is a $\xi \in B$ satisfying $\xi^{*}=\xi$ so that the $*$-symmetric pairing $(-,-)$ associated to the alternating form $\langle-,-\rangle$ of Lemma 5.1 .3 is given by

$$
(x, y)=\operatorname{Tr}_{B / F}\left(x \xi y^{*}\right) .
$$

Proof. The element $\xi$ is computed to be $2 \delta \beta$.

For a non-degenerate $*$-hermitian $*$-symmetric form $(-,-)$, we define the discriminant

$$
\operatorname{disc}=N_{B / F}(\xi) \in \mathbb{Q}^{\times} / N_{F / \mathbb{Q}}\left(F^{\times}\right)
$$

for $\xi$ the element of Corollary 5.1.4. The discriminant is an invariant of the isometry class of $(-,-)$.

Two involutions $\iota$ and $\iota^{\prime}$ are equivalent if there exists a $c \in C^{\times}$so that

$$
c x^{\iota} c^{-1}=\left(c x c^{-1}\right)^{\iota^{\prime}} .
$$

We finish this section by explaining how the classification of involutions up to equivalence is related to the classification of hermitian forms.

Proposition 5.1.5. The association of an involution on $C$ with a non-degenerate *-hermitian alternating form on $V$ establishes a bijective correspondence

$\{$ Similitude classes of non-degenerate $*$-hermitian alternating forms on $V$ \}

$\{$ Equivalence classes of involutions of the second kind on $C\}$

We first need the following lemma.

Lemma 5.1.6. Let $\iota$ be an involution of the second kind on $C$. Then there exists an element $\gamma \in C^{\times}$satisfying $\gamma^{*}=-\gamma$ which gives ८ by the formula

$$
x^{\iota}=\gamma^{-1} x^{*} \gamma .
$$

The element $\gamma$ is unique up to multiplication by an element in $\mathbb{Q}^{\times}$.

Proof. The Noether-Skolem theorem implies that there exists an element $\alpha \in$ $C^{\times}$, unique up to $F^{\times}$-multiple, such that

$$
\left(x^{*}\right)^{\iota}=\alpha^{-1} x \alpha
$$

for all $x \in C$. Therefore, we have $x^{\iota}=\alpha^{-1} x^{*} \alpha$. Since $\iota$ is an involution, we determine that $\alpha^{-1} \alpha^{*}=c \in F^{\times}$. By Hilbert's Theorem 90 there exists an $a \in F^{\times}$ so that $\gamma=a \alpha$ satisfies $\gamma^{*}=-\gamma$. Such an element $\gamma$ is determined up to a multiple in $\mathbb{Q}^{\times}$. 
Proof of Proposition 5.1.5. By Lemma 5.1.3, given a non-degenerate $*-$ hermitian alternating form $\langle-,-\rangle$, there exists an element $\beta$ such that

$$
\langle x, y\rangle=\langle x, y\rangle_{\beta}=\operatorname{Tr}_{F / \mathbb{Q}} \operatorname{Tr}_{B / F}\left(x \beta y^{*}\right) .
$$

Letting $\gamma$ be the element $\beta$ regarded as an element of $C=B^{o p}$, the associated involution $\iota_{\gamma}$ is given by $x^{\iota_{\gamma}}=\gamma^{-1} x^{*} \gamma$. Changing $\langle-,-\rangle_{\beta}$ by a $\mathbb{Q}^{\times}$-multiple changes $\beta$ and $\gamma$ by a $\mathbb{Q}^{\times}$-multiple, leaving the involution $\iota_{\gamma}$ unchanged. In the other direction, Lemma 5.1.6 associates to an involution $\iota$ an element $\gamma \in C^{\times}$, unique up to $\mathbb{Q}^{\times}$-multiple, so that

$$
x^{\iota}=x^{\iota \gamma}=\gamma^{-1} x^{*} \gamma
$$

and $\gamma^{*}=-\gamma$. Letting $\beta$ be the element $\gamma$ regarded as an element of $B$, we can then associate the similitude class of form $\langle-,-\rangle_{\beta}$. Forms $\langle-,-\rangle_{\beta}$ and $\langle-,-\rangle_{\beta^{\prime}}$ lie in the same similitude class if and only if the associated involutions $\iota_{\gamma}$ and $\iota_{\gamma^{\prime}}$ are equivalent.

\subsection{Unitary and similitude groups}

We now fix the following:

$$
\begin{aligned}
\langle-,-\rangle & =\text { non-degenerate } * \text {-hermitian alternating pairing on } V, \\
(-,-) & =\text { corresponding } * \text {-symmetric pairing, } \\
(-)^{\iota} & =\text { involution on } C \text { defined by }\langle c v, w\rangle=\left\langle v, c^{\iota} w\right\rangle .
\end{aligned}
$$

Associated to this pairing are some group-schemes defined over $\mathbb{Q}$ : the unitary group $U$ and the similitude group $G U$. For a $\mathbb{Q}$-algebra $R$, the $R$-points of these groups are given by

$$
\begin{aligned}
U(R) & =\left\{g \in(C \otimes R)^{\times}: g^{\iota} g=1\right\}, \\
G U(R) & =\left\{g \in(C \otimes R)^{\times}: g^{\iota} g \in R^{\times}\right\} .
\end{aligned}
$$

Let $V_{R}$ denote the $C \otimes R$-module $V \otimes R$. The $R$-points of $U$ (respectively, $G U$ ) consist of the isometries (respectively similitudes) of both pairings $(-,-)$ and $\langle-,-\rangle$ on $V_{R}$. The group $G U$ has a similitude norm

$$
\nu: G U \rightarrow \mathbb{G}_{m}
$$

which, on $R$-points, takes an element $g$ to the quantity $g^{l} g \in R^{\times}$. For $v, w \in V_{R}$, we have

$$
\langle g v, g w\rangle=\nu(g)\langle v, w\rangle .
$$

Let $G L_{C}$ denote the form of $G L_{n}$ over $F$ whose $R$ points are given by

$$
G L_{C}(R)=\left(C \otimes_{F} R\right)^{\times} .
$$

LEMMA 5.2.1. There are natural isomorphisms of group schemes over F:

$$
\begin{aligned}
\operatorname{Spec}(F) \times_{\operatorname{Spec}(\mathbb{Q})} U & \cong G L_{C}, \\
\operatorname{Spec}(F) \times_{\operatorname{Spec}(\mathbb{Q})} G U & \cong G L_{C} \times \mathbb{G}_{m} .
\end{aligned}
$$


Proof. It suffices to provide a natural isomorphism on $R$-points for $F$-algebras $R$. Since $R$ is an $F$-algebra, there is a decomposition

$$
\begin{aligned}
C \otimes_{\mathbb{Q}} R & \cong C \otimes_{F}\left(F \otimes_{\mathbb{Q}} R\right) \\
& \cong\left(C \otimes_{F} R\right) \times\left(C \otimes_{F, c} R\right) .
\end{aligned}
$$

The induced involution $\iota$ on $\left(C \otimes_{F} R\right) \times\left(C \otimes_{F, c} R\right)$ is given by

$$
\left(c_{1} \otimes r_{1}, c_{2} \otimes r_{2}\right)^{\iota}=\left(c_{2}^{\iota} \otimes r_{2}, c_{1}^{\iota} \otimes r_{1}\right) .
$$

Therefore we have a natural isomorphism

$$
\begin{aligned}
U(R) & =\left\{g \in\left(C \otimes_{\mathbb{Q}} R\right)^{\times}: g^{\iota} g=1\right\} \\
& \cong\left\{\left(g_{1}, g_{2}\right) \in\left(C \otimes_{F} R\right)^{\times} \times\left(C \otimes_{F, c} R\right)^{\times}: g_{2}^{\iota} g_{1}=1\right\} \\
& \cong\left\{\left(g_{1}, g_{1}^{-\iota}\right): g_{1} \in\left(C \otimes_{F} R\right)^{\times}\right\} \\
& \cong G L_{C}(R) .
\end{aligned}
$$

Similarly, there are natural isomorphisms

$$
\begin{aligned}
G U(R) & =\left\{g \in\left(C \otimes_{\mathbb{Q}} R\right)^{\times}: g^{\iota} g=\nu \in R^{\times}\right\} \\
& \cong\left\{\left(g_{1}, g_{1}^{-\iota} \nu\right): g_{1} \in\left(C \otimes_{F} R\right)^{\times}, v \in R^{\times}\right\} \\
& \cong G L_{C}(R) \times \mathbb{G}_{m}(R) .
\end{aligned}
$$

\subsection{Classification of forms}

Let $K$ be either $\mathbb{Q}$ or $\mathbb{Q}_{x}$ for some place $x$ of $\mathbb{Q}$. The classification of our various structures are parameterized by certain Galois cohomology groups:

$$
\begin{aligned}
H^{1}(K, U) & =\left\{\begin{array}{l}
\text { Isometry classes of non-degenerate } \\
* \text {-hermitian alternating forms on } V_{K}
\end{array}\right\}, \\
H^{1}(K, G U) & =\left\{\begin{array}{l}
\text { Similitude classes of non-degenerate } \\
* \text {-hermitian alternating forms on } V_{K}
\end{array}\right\} \\
& =\left\{\begin{array}{l}
\text { Equivalence classes of involutions } \\
\text { of the second kind on } C \otimes K
\end{array}\right\} .
\end{aligned}
$$

Local case where $x$ is finite and split. Let $x$ split as $y y^{c}$. Then $\mathbb{Q}_{x}$ is an $F$-algebra, and hence by Lemma 5.2.1, there are isomorphisms of group schemes

$$
\begin{aligned}
\operatorname{Spec}\left(\mathbb{Q}_{x}\right) \times_{\operatorname{Spec}(\mathbb{Q})} U & \cong G L_{C_{y}} \\
\operatorname{Spec}\left(\mathbb{Q}_{x}\right) \times_{\operatorname{Spec}(\mathbb{Q})} G U & \cong G L_{C_{y}} \times \mathbb{G}_{m}
\end{aligned}
$$

If $C$ is a central simple algebra over $\mathbb{Q}_{x}$, it is a well-known generalization of Hilbert's Theorem 90 that $H^{1}\left(\mathbb{Q}_{x}, G L_{C}\right)=0$ (see, for instance, the proof of [Mil05, Prop. 26.6]). We therefore have:

Lemma 5.3.1. If $x$ is split in $F$, then we have $H^{1}\left(\mathbb{Q}_{x}, U\right)=H^{1}\left(\mathbb{Q}_{x}, G U\right)=0$. 
Local case where $x$ is infinite. Because $F$ is imaginary, $C$ is necessarily split at $x=\infty$. The computation of $H^{1}(\mathbb{R}, U)$ and $H^{1}(\mathbb{R}, G U)$ is then given by the classification of hermitian forms $(-,-)^{\prime}$ on $W \cong \mathbb{C}^{n}$. These forms are classified up to isometry by their signature, and up to similitude by the absolute value of their signature. We therefore have the following lemma.

LEMma 5.3.2. The signature induces isomorphisms

$$
\begin{aligned}
H^{1}(\mathbb{R}, U) & \cong\{(p, q): p+q=n\}, \\
H^{1}(\mathbb{R}, G U) & \cong\{\{p, q\}: p+q=n\} .
\end{aligned}
$$

Local case where $x$ is finite and not split. Analogously to the global case, relative to the involution $*$ we may associate to a form $(-,-)$ on $V_{x}$ its discriminant disc $\in \mathbb{Q}_{x}^{\times} / N\left(F_{x}^{\times}\right) \cong \mathbb{Z} / 2$. A proof of the following lemma may be found in [Sch85].

LEMMA 5.3.3. If $x$ does not split in $F$, the discriminant gives an isomorphism

$$
H^{1}\left(\mathbb{Q}_{x}, U\right) \cong \mathbb{Z} / 2 \text {. }
$$

COROLlary 5.3.4. If $x$ does not split in $F$, there are isomorphisms

$$
H^{1}\left(\mathbb{Q}_{x}, G U\right)= \begin{cases}\mathbb{Z} / 2, & n \text { even }, \\ 0, & n \text { odd } .\end{cases}
$$

Proof. Let $a \in \mathbb{Q}^{\times}$represent a generator of $\mathbb{Q}^{\times} / N\left(F_{x}^{\times}\right)$. If $n$ is even, the discriminant of $a(-,-)$ is equal to that of $(-,-)$. If $n$ is odd, than the discriminant of $a(-,-)$ is not equal to that of $(-,-)$.

Global case. For places $x$ of $\mathbb{Q}$, we define maps

$$
\begin{array}{ll}
\xi_{x}: H^{1}\left(\mathbb{Q}_{x}, U\right) \rightarrow \mathbb{Z} / 2, \\
\xi_{x}^{\prime}: H^{1}\left(\mathbb{Q}_{x}, G U\right) \rightarrow \mathbb{Z} / 2, \quad n \text { even. }
\end{array}
$$

If $x$ is finite and split, $H^{1}\left(\mathbb{Q}_{x}, U\right)=H^{1}\left(\mathbb{Q}_{x}, G U\right)=0$. If $x$ is finite and not split in $F$, let $\xi_{x}, \xi_{x}^{\prime}$ be the unique isomorphisms

$$
\begin{aligned}
& \xi_{x}: H^{1}\left(\mathbb{Q}_{x}, U\right) \stackrel{\text { disc }}{\cong} \mathbb{Q}_{x}^{\times} / N\left(F_{x}^{\times}\right) \cong \mathbb{Z} / 2, \\
& \xi_{x}^{\prime}: H^{1}\left(\mathbb{Q}_{x}, G U\right) \stackrel{\text { disc }}{\cong} \mathbb{Q}_{x}^{\times} / N\left(F_{x}^{\times}\right) \cong \mathbb{Z} / 2, \quad n \text { even. }
\end{aligned}
$$

If $n$ is odd we have $H^{1}\left(\mathbb{Q}_{x}, G U\right)=0$. At the infinite place, we define:

$$
\begin{aligned}
\xi_{\infty}((p, q)) & \equiv q \quad(\bmod 2), \\
\xi_{\infty}^{\prime}(\{p, q\}) & \equiv p \equiv q \quad(\bmod 2), \quad n \text { even. }
\end{aligned}
$$

The following result may be translated from the classification of global hermitian forms given in [Sch85] (see also [Clo91]).

TheOREM 5.3.5. There is a short exact sequence

$$
0 \rightarrow H^{1}(\mathbb{Q}, U) \rightarrow \bigoplus_{x} H^{1}\left(\mathbb{Q}_{x}, U\right) \stackrel{\sum \xi_{x}}{\longrightarrow} \mathbb{Z} / 2 \rightarrow 0 .
$$


Proof. Scharlau [Sch85] shows that non-degenerate $*$-symmetric $*$-hermitian forms on $V$ are classified by their discriminant disc $\in \mathbb{Q}^{\times} / N\left(F^{\times}\right)$and signature $(p, q)$. Global properties of the Hilbert symbol give rise to a short exact sequence

$$
0 \rightarrow \mathbb{Q}^{\times} / N\left(F^{\times}\right) \rightarrow \bigoplus_{x} \mathbb{Q}_{x}^{\times} / N\left(F_{x}^{\times}\right) \stackrel{\sum_{x}}{\longrightarrow} \mathbb{Z} / 2 \rightarrow 0 .
$$

The result follows from the local computations, together with the obvious relation in $\mathbb{R}^{\times} / N\left(\mathbb{C}^{\times}\right) \cong \mathbb{Z} / 2$ :

$$
\operatorname{disc}_{\infty} \equiv q \quad(\bmod 2) .
$$

COROLLARY 5.3.7. If $n$ is even, then there is a short exact sequence

$$
0 \rightarrow H^{1}(\mathbb{Q}, G U) \rightarrow \bigoplus_{x} H^{1}\left(\mathbb{Q}_{x}, G U\right) \stackrel{\sum \xi_{x}^{\prime}}{\longrightarrow} \mathbb{Z} / 2 \rightarrow 0 .
$$

If $n$ is odd then the absolute value of the signature gives an isomorphism

$$
H^{1}(\mathbb{Q}, G U) \stackrel{\cong}{\rightrightarrows} H^{1}(\mathbb{R}, G U) .
$$

Proof. If a form $(-,-)$ has discriminant disc, the form $r(-,-)$ has discriminant $r^{n} \cdot \operatorname{disc}$, for $r \in \mathbb{Q}^{\times}$. Since $\left(\mathbb{Q}^{\times}\right)^{2}$ is contained in $N\left(F^{\times}\right)$, if $n$ is even the discriminant is an invariant of the similitude class of $(-,-)$. However, if $n$ is odd, then the short exact sequence (5.3.6) shows that there exists a global multiple $r$ which kills the local invariants $\operatorname{disc}_{x}$ for finite places $x$, with the effect of possibly changing the sign of the signature. 



\section{CHAPTER 6}

\section{Shimura varieties of type $U(1, n-1)$}

In this chapter we describe the Shimura stacks we wish to study. The integral version described here is essentially due to Kottwitz, and the exposition closely follows [Kot92], [HT01], and [Hid04].

\subsection{Motivation}

In Section 2.2, we saw that the simplest examples of abelian varieties $A$ over $\overline{\mathbb{F}}_{p}$, whose $p$-divisible group $A(p)$ contains a 1-dimensional summand of slope $1 / n$, had dimension $n$ and complex multiplication by $F$, a quadratic imaginary extension of $\mathbb{Q}$ in which $p$ splits. For such abelian varieties, the 1-dimensional summand of $A(p)$ is given by $A(u)$, where $u$ is a prime of $F$ dividing $p$.

Let $(A, i)$ be an $n^{2}$-dimensional $M_{n}(F)$-linear abelian variety. The action of $M_{n}(F)$ makes $A$ isogenous to $A_{0}^{n}$, where $A_{0}$ is an $n$-dimensional abelian variety with complex multiplication by $F$. Thus there is an equivalence between the quasi-isogeny category of $n$-dimensional abelian varieties $A_{0}$ with complex multiplication by $F$ and the quasi-isogeny category of $n^{2}$ dimensional $M_{n}(F)$-linear abelian varieties $(A, i)$.

More generally, we saw in Section 2.2 that if $B$ is any central simple algebra over $F$ of dimension $n^{2}$, then there exist simple $B$-linear abelian varieties with $A(u)$ of dimension $n$ and slope $1 / n$. The $B$-linear structure on $A$ induces a $B_{u}$-linear structure on $A(u)$. If we assume that $B$ is split over $u$, then the $p$-divisible group $A(u)$ is isogenous to a product $(\epsilon A(u))^{n}$, where $\epsilon$ is an idempotent of $B_{u}=M_{n}\left(F_{u}\right)$ and $\epsilon A(u)$ is a 1-dimensional $p$-divisible group of slope $1 / n$.

Let $*$ be a positive involution on $B$. Introducing the structure of a compatible polarization $\lambda$ on our $n^{2}$-dimensional $B$-linear abelian varieties, one could form a moduli stack of tuples $(A, i, \lambda)$ for which the $p$-divisible group $A(u)$ is $n$-dimensional.

These moduli stacks will, in general, have infinitely many components. However, Lemma 4.6.3 indicates that the classification of such polarizations is controlled by the local similitude classes of $\lambda$-Weil pairings on the Tate modules $V_{\ell}(A)$. We may pick out finitely many components of our moduli space by fixing a global pairing $\langle-,-\rangle$, and restricting our attention to only those tuples $(A, i, \lambda)$ for which the Weil pairings $\lambda\langle-,-\rangle$ on $V_{\ell}(A)$ are similar to the local pairing $\langle-,-\rangle_{\ell}$ for each $\ell \neq p$. Such moduli stacks are instances of Shimura stacks. 


\subsection{Initial data}

Begin with the following data.

$$
\begin{aligned}
F= & \text { quadratic imaginary extension of } \mathbb{Q} \text {, such that } p \text { splits as } u u^{c} . \\
\mathcal{O}_{F}= & \text { ring of integers of } F . \\
B= & \text { central simple algebra over } F, \operatorname{dim}_{F} B=n^{2}, \text { split over } u \text { and } u^{c} . \\
(-)^{*}= & \text { positive involution on } B \text { of the second kind, i.e.: } \\
& 1 . \operatorname{Tr}_{F / \mathbb{Q}} \operatorname{Tr}_{B / F}\left(x x^{*}\right)>0 \text { for } x \neq 0 . \\
& \quad 2 . * \text { restricts to conjugation on } F . \\
\mathcal{O}_{B}= & \text { maximal order in } B \text { such that } \mathcal{O}_{B,(p)} \text { is preserved under } * . \\
V= & \text { free left } B \text {-module. } \\
\langle-,-\rangle= & \mathbb{Q} \text {-valued non-degenerate alternating form on } V \\
& \quad \text { which is } * \text {-hermitian. (This means }\langle\alpha x, y\rangle=\left\langle x, \alpha^{*} y\right\rangle . \\
L= & \mathcal{O}_{B} \text {-lattice in } V,\langle-,-\rangle \text { restricts to give integer values on } L, \\
& \quad \text { and makes } L_{(p)} \text { self-dual. }
\end{aligned}
$$

From this data we define:

$$
\begin{aligned}
L^{p} & =\prod_{\ell \neq p} L \otimes \mathbb{Z}_{\ell}, \\
V^{p} & =V \otimes_{\mathbb{Q}} \mathbb{A}^{p, \infty}, \\
C & =\operatorname{End}_{B}(V), \\
(-)^{\iota} & =\text { involution on } C \text { defined by }\langle a v, w\rangle=\left\langle v, a^{\iota} w\right\rangle, \\
\mathcal{O}_{C} & =\text { order of elements } x \in C \text { such that } x(L) \subseteq L, \\
G U(R) & =\left\{g \in\left(C \otimes_{\mathbb{Q}} R\right)^{\times}: g^{\iota} g \in R^{\times}\right\}, \\
U(R) & =\left\{g \in\left(C \otimes_{\mathbb{Q}} R\right)^{\times}: g^{\iota} g=1\right\}, \\
K_{0}^{p} & =\left\{g \in G U\left(\mathbb{A}^{p, \infty}\right): g\left(L^{p}\right)=L^{p}\right\} .
\end{aligned}
$$

We are interested in the case where we have:

$$
\begin{aligned}
V & =B, \\
L & =\mathcal{O}_{B}, \\
U(\mathbb{R}) & \cong U(1, n-1) .
\end{aligned}
$$

It then follows that we have

$$
\begin{aligned}
C & \cong B^{o p}, \\
\mathcal{O}_{C} & \left.\cong \mathcal{O}_{B}^{o p} \quad \text { (by maximality of } \mathcal{O}_{B}\right) .
\end{aligned}
$$

In our case $*$ may equally well be regarded as an involution $*$ on $C$, and there exists (Lemma 5.1.3) an element $\beta \in B$ satisfying $\beta^{*}=-\beta$ which encodes $\langle-,-\rangle$ :

$$
\langle x, y\rangle=\operatorname{Tr}_{F / \mathbb{Q}} \operatorname{Tr}_{B / F}\left(x \beta y^{*}\right) .
$$


Let $\gamma$ be the element $\beta$ regarded as an element of $C$. Then $\iota$ is given by

$$
z^{\iota}=\gamma^{-1} z^{*} \gamma
$$

Tensoring with $\mathbb{R}$, and taking the complex embedding of $F$ which sends $\delta$ to a negative multiple of $i$, we may identify the completions of our simple algebras with matrix algebras over $\mathbb{C}$ :

$$
\begin{aligned}
& B_{\infty}=M_{n}(\mathbb{C}), \\
& *=\text { conjugate transpose, } \\
& C_{\infty}=M_{n}(\mathbb{C}) \quad \text { (identified with } B \text { through the transpose), } \\
& \beta=\left[\begin{array}{cccc}
e_{1} i & & & \\
& -e_{2} i & & \\
& & \ddots & \\
& & & -e_{n} i
\end{array}\right] .
\end{aligned}
$$

Here the $e_{i}$ 's are positive real numbers.

Because $B$ was assumed to be split over $u$, we may fix an isomorphism

$$
\mathcal{O}_{B, u} \cong M_{n}\left(\mathcal{O}_{F, u}\right)=M_{n}\left(\mathbb{Z}_{p}\right) \text {. }
$$

Let $\epsilon \in \mathcal{O}_{B, u}$ be the projection associated by this isomorphism to the matrix which has a 1 in the $(1,1)$ entry, and zeros elsewhere.

REMARK 6.2.1. Giving the data $\left(B,(-)^{*},\langle-,-\rangle\right)$ is essentially the same as specifying a form of the similitude group $G U$. The forms of $G U$ are classified by the Galois cohomology group $H^{1}(\mathbb{Q}, P G U)$, where the algebraic group $P G U$ is the quotient of $G U$ by the subgroup $T_{F}$, where

$$
T_{F}(R)=\left(F \otimes_{\mathbb{Q}} R\right)^{\times} .
$$

Clozel describes this computation in [Clo91]: an element in $H^{1}(\mathbb{Q}, P G U)$ corresponds uniquely to the local invariants of the division algebra $B$, as well as the difference between the classes of $H^{1}(\mathbb{Q}, G U)$ determined by the involution $*$ and the pairing $\langle-,-\rangle$ (see Section 5.3).

\subsection{Statement of the moduli problem}

Assume that $S$ is a scheme, and that $A$ is an abelian scheme over $S$. A polarization $\lambda: A \rightarrow A^{\vee}$ of $A$ is an isogeny which restricts to a polarization on each of the geometric fibers of $A / S$. For $R$ a ring contained in $\mathbb{R}$, an $R$-polarization is an $R$-isogeny

$$
\lambda \in \operatorname{Hom}\left(A, A^{\vee}\right) \otimes R
$$

which is a positive linear combination of polarizations. A $\mathbb{Q}$-polarization defines a $\lambda$-Rosati involution $\dagger$ on $\operatorname{End}^{0}(A)$ by $f^{\dagger}=\lambda^{-1} f^{\vee} \lambda$.

We shall define two functors

$$
\mathcal{X}, \mathcal{X}^{\prime}:\left\{\text { locally noetherian formal schemes/ } \operatorname{Spf}\left(\mathbb{Z}_{p}\right)\right\} \rightarrow \text { groupoids. }
$$

We shall then show that these functors are equivalent. 
REMARK 6.3.1. Every formal scheme $S$ over $\operatorname{Spf}\left(\mathbb{Z}_{p}\right)$ is a formal colimit $S=\lim S_{i}$, where $S_{i}$ is a scheme on which $p$ is locally nilpotent. It therefore suffices to define the functors $\mathcal{X}$ and $\mathcal{X}^{\prime}$ for locally noetherian schemes on which $p$ is locally nilpotent.

The functor $\mathcal{X}$. Assume that $S$ is a locally noetherian scheme on which $p$ is locally nilpotent. The objects of the groupoid $\mathcal{X}(S)$ consist of tuples of data $(A, i, \lambda)$ as follows.

$$
\begin{array}{ll}
A & \text { is an abelian scheme over } S \text { of dimension } n^{2} . \\
\lambda: A \rightarrow A^{\vee} & \text { is a } \mathbb{Z}_{(p)} \text {-polarization, with Rosati involution } \dagger \text { on } \\
& \text { End }(A)_{(p)} \text {. } \\
i: \mathcal{O}_{B,(p)} \hookrightarrow \operatorname{End}(A)_{(p)} & \text { is an inclusion of rings, satisfying } i\left(b^{*}\right)=i(b)^{\dagger} \text { in } \\
& \operatorname{End}(A)_{(p)}, \text { such that } \epsilon A(u) \text { is 1-dimensional. }
\end{array}
$$

We impose the following additional restrictions on $(A, i, \lambda)$. Choose a geometric point $s$ in each component of $S$. We require that for each of these points there exists an $\mathcal{O}_{B}$-linear integral uniformization

$$
\eta: L^{p} \stackrel{\cong}{\longrightarrow} T^{p}\left(A_{s}\right)
$$

so that when tensored with $\mathbb{Q}, \eta$ sends $\langle-,-\rangle$ to an $\left(\mathbb{A}^{p, \infty}\right)^{\times}$-multiple of the $\lambda$-Weil pairing. We do not fix this uniformization as part of the data.

We pause to explain this last restriction. By Lemma 4.6.3, the isogeny classes of compatible polarizations $\lambda$ on a fixed $B$-linear abelian variety $A$ over an algebraically closed field $k$ are determined by the elements of $H^{1}\left(\mathbb{Q}_{\ell}, G U\right)$ given at each $\ell \neq p$ by the $\lambda$-Weil pairing on $V_{\ell}(A)$. The existence of the isomorphism $\eta$ is independent of the point $s$.

A morphism

$$
(A, i, \lambda) \rightarrow\left(A^{\prime}, i^{\prime}, \lambda^{\prime}\right)
$$

in $\mathcal{X}(S)$ consists of an isomorphism of abelian schemes over $S$

$$
\alpha: A \stackrel{\cong}{\longrightarrow} A^{\prime}
$$

such that

$$
\begin{aligned}
\lambda & =r \alpha^{\vee} \lambda^{\prime} \alpha, & r & \in \mathbb{Z}_{(p)}^{\times}, \\
i^{\prime}(b) \alpha & =\alpha i(b), & b & \in \mathcal{O}_{B} .
\end{aligned}
$$

In particular, the isomorphism class of $(A, i, \lambda)$ depends only on the weak polarization determined by $\lambda$.

The functor $\mathcal{X}^{\prime}$. The functor $\mathcal{X}$ classifies $\mathcal{O}_{B}$-linear polarized abelian varieties up to isomorphism (with certain restrictions on the slopes of the $p$-divisible group and the Weil pairings). We shall now introduce a different functor $\mathcal{X}^{\prime}$ which classifies $\mathcal{O}_{B,(p)}$-linear polarized abelian varieties with rational level structure up to isogeny. The functor $\mathcal{X}^{\prime}$ will be shown to be equivalent to $\mathcal{X}$. 
Assume that $S$ is a locally noetherian connected scheme on which $p$ is locally nilpotent. Fix a geometric point $s$ of $S$. The objects of the groupoid $\mathcal{X}^{\prime}(S)$ consist of tuples of data $(A, i, \lambda,[\eta])$ as follows.

$\begin{array}{ll}A & \text { is an abelian scheme over } S \text { of dimension } n^{2} . \\ \lambda: A \rightarrow A^{\vee} & \text { is a } \mathbb{Z}_{(p)} \text {-polarization, with Rosati involution } \dagger \text { on } \\ & \text { End }(A)_{(p)} \text {. } \\ i: \mathcal{O}_{B,(p)} \hookrightarrow \operatorname{End}(A)_{(p)} & \text { is an inclusion of rings, satisfying } i\left(b^{*}\right)=i(b)^{\dagger} \text { in } \\ & \text { End }(A)_{(p)}, \text { such that } \epsilon A(u) \text { is } 1 \text {-dimensional. } \\ & \text { is a rational } K_{0}^{p} \text { level structure, i.e. the } K_{0}^{p} \text {-orbit } \\ & \text { of a rational uniformization } \eta: V^{p} \cong V^{p}\left(A_{s}\right) \text {, such } \\ & \text { that } \eta \text { is } \mathcal{O}_{B,(p)} \text {-linear, }[\eta] \text { is } \pi_{1}(S, s) \text {-invariant, and } \\ & \eta \text { sends }\langle-,-\rangle \text { to an }\left(\mathbb{A}^{p, \infty}\right)^{\times} \text {-multiple of the } \lambda \text {-Weil } \\ & \text { pairing. }\end{array}$

A morphism

$$
(A, i, \lambda, \eta) \rightarrow\left(A^{\prime}, i^{\prime}, \lambda^{\prime}, \eta^{\prime}\right)
$$

in $\mathcal{X}^{\prime}(S)$ consists of a $\mathbb{Z}_{(p)}$-isogeny of abelian schemes over $S$

$$
\alpha: A \stackrel{\simeq}{\leftrightarrows} A^{\prime}
$$

such that

$$
\begin{array}{rlrl}
\lambda & =r \alpha^{\vee} \lambda^{\prime} \alpha, \quad r \in \mathbb{Z}_{(p)}^{\times}, \\
i^{\prime}(b) \alpha & =\alpha i(b), & b \in \mathcal{O}_{B,(p)}, \\
{\left[\eta^{\prime}\right]} & =\alpha_{*}[\eta] . & &
\end{array}
$$

The $\pi_{1}(S, s)$-invariance of the level structure $[\eta]$ implies that the objects are independent of the choice of the point $s$ in $S$. The functor $\mathcal{X}^{\prime}$ extends to schemes which are not connected by taking products over the values on the components.

\subsection{Equivalence of the moduli problems}

Fix a locally noetherian connected scheme $S$ on which $p$ is locally nilpotent, with geometric point $s$ as before. For each object $(A, i, \lambda)$ of $\mathcal{X}(S)$, choose an $\mathcal{O}_{B}$-linear similitude

$$
\eta: L^{p} \cong T^{p}\left(A_{s}\right) .
$$

Then $\left(A, i_{(p)}, \lambda,\left[\eta_{\mathbb{Q}}\right]\right)$ is an object of $\mathcal{X}^{\prime}(S)$. Different choices of $\eta$ yield canonically equivalent objects $\left(A, i_{(p)}, \lambda,\left[\eta_{\mathbb{Q}}\right]\right)$, because any two choices of $\eta$ will necessarily differ by an element of $K_{0}^{p}$. Thus we have produced a functor

$$
F_{S}: \mathcal{X}(S) \rightarrow \mathcal{X}^{\prime}(S)
$$

which is natural in $S$. The rest of this section will be devoted to proving the following theorem.

TheOREM 6.4.1. The functor $F_{S}$ is an equivalence of categories. 
$F_{S}$ is essentially surjective. Suppose that $(A, i, \lambda,[\eta])$ is an object of $\mathcal{X}^{\prime}(S)$. In particular, choose a rational uniformization $\eta$. We need to show that this data is isogenous to a new set of data $\left(A^{\prime}, i^{\prime}, \lambda^{\prime},\left[\eta^{\prime}\right]\right)$ where $\eta^{\prime}$ lifts to an integral level structure.

Define $L_{s}$ to be the virtual subgroup $\kappa(\eta) \in \operatorname{VSub}^{p}\left(A_{s}\right)$ of Section 3.3. The $\pi_{1}(S, s)$ invariance of $[\eta]$ implies that this virtual subgroup is invariant under $\pi_{1}(S, s)$. Therefore, the virtual subgroup $L_{s}$ extends to a local system of virtual subgroups $L$ of $A$. Explicitly, there exists an integer $N$ prime to $p$ such that $N^{-1} L_{s}$ corresponds to an actual subgroup scheme $H_{s}$ of $A_{s}$, and a finite subgroup scheme $H$ of $A$ over $S$ extending $H_{s}$. Define $A^{\prime}$ to be the quotient abelian variety, with quotient isogeny

$$
\alpha^{\prime}: A \rightarrow A / H=A^{\prime} .
$$

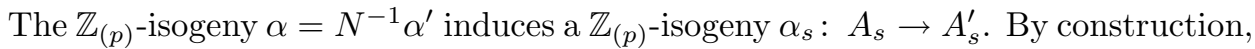
$\alpha_{s}\left(L_{s}\right)=T^{p}\left(A_{s}^{\prime}\right)$.

The $\mathbb{Z}_{(p)}$-polarization $\lambda^{\prime}$ on $A^{\prime}$ is defined to be the $\mathbb{Z}_{(p)}$-isogeny that makes the following diagram commute.

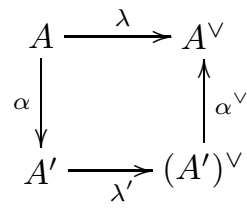

Define $\mathcal{O}_{B,(p)}$-multiplication on $A^{\prime}$

$$
i^{\prime}: \mathcal{O}_{B,(p)} \hookrightarrow \operatorname{End}\left(A^{\prime}\right)_{(p)}
$$

by the formula $i^{\prime}(b)=\alpha i(b) \alpha^{-1}$. Compatibility of $i^{\prime}$ with the $\lambda^{\prime}$-Rosati involution $\dagger^{\prime}$ is easily checked:

$$
\begin{aligned}
i^{\prime}(b)^{\dagger^{\prime}} & =\left(\lambda^{\prime}\right)^{-1} i^{\prime}(b)^{\vee} \lambda^{\prime} \\
& =\left(\left(\alpha^{\vee}\right)^{-1} \lambda \alpha^{-1}\right)^{-1}\left(\alpha i(b) \alpha^{-1}\right)^{\vee}\left(\left(\alpha^{\vee}\right)^{-1} \lambda \alpha^{-1}\right) \\
& =\alpha \lambda^{-1} i(b)^{\vee} \lambda \alpha^{-1} \\
& =\alpha i(b)^{\dagger} \alpha^{-1} \\
& =\alpha i\left(b^{*}\right) \alpha^{-1} \\
& =i^{\prime}\left(b^{*}\right) .
\end{aligned}
$$

The rational level structure $\left[\eta^{\prime}\right]$ is defined to be the the orbit of the composite

$$
\eta^{\prime}: V^{p} \stackrel{\eta}{\rightarrow} V^{p}\left(A_{s}\right) \stackrel{\alpha_{s}}{\longrightarrow} V^{p}\left(A_{s}^{\prime}\right) .
$$

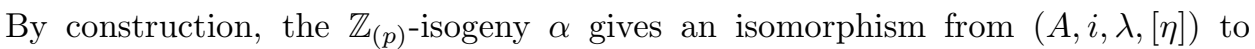
$\left(A^{\prime}, i^{\prime}, \lambda^{\prime},\left[\eta^{\prime}\right]\right)$. We wish to show that $\eta^{\prime}$ lifts to an integral uniformization. By Lemma 3.3.1, it suffices to show that the virtual subgroup $\kappa\left(\eta^{\prime}\right) \in \operatorname{VSub}^{p}\left(A_{s}^{\prime}\right)$ is $T^{p}\left(A_{s}\right)$.

However, by definition $\kappa\left(\eta^{\prime}\right)=\alpha_{s}\left(\eta\left(L^{p}\right)\right)=\alpha_{s}\left(L_{s}\right)$, and we have already shown that $\alpha_{s}\left(L_{s}\right)=T^{p}\left(A_{s}\right)$. 
We will have shown that $\left(A^{\prime}, i^{\prime}, \lambda^{\prime}\right)$ may be regarded as an object of $\mathcal{X}(S)$, provided that the $\mathcal{O}_{B,(p)}$-multiplication on $\operatorname{End}\left(A^{\prime}\right)_{(p)}$ given by $i^{\prime}$ lifts to $\mathcal{O}_{B}$-multiplication on $\operatorname{End}\left(A^{\prime}\right)$. The existence of an $\mathcal{O}_{B}$-action on $A^{\prime}$ follows from the following pair of pullback squares (the righthand square is a pullback by Corollary 3.5.3).

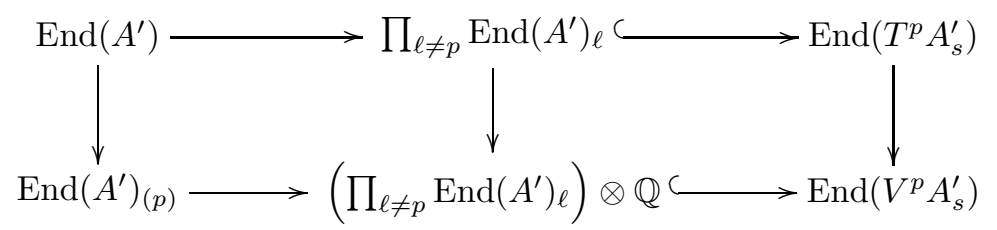

$F_{S}$ is fully faithful. The faithfulness of $F_{S}$ is obvious. We just need to show that it is full. Suppose that $(A, \lambda, i, \eta)$ and $\left(A^{\prime}, \lambda^{\prime}, i^{\prime}, \eta^{\prime}\right)$ are two objects of $\mathcal{X}^{\prime}(S)$ which are in the image of $F_{S}$. Without loss of generality assume that the rational level structures $\eta$ and $\eta^{\prime}$ lift to integral level structures. Suppose that

$$
f:(A, \lambda, i, \eta) \rightarrow\left(A^{\prime}, \lambda^{\prime}, i^{\prime}, \eta^{\prime}\right)
$$

is a morphism in $\mathcal{X}^{\prime}(S)$. In particular, $f: A \rightarrow A^{\prime}$ is a $\mathbb{Z}_{(p)}$-isogeny. Because $\eta$ and $\eta^{\prime}$ are integral level structures, we may conclude that the induced map

$$
f_{*}: V^{p}(A) \stackrel{\cong}{\longrightarrow} V^{p}\left(A^{\prime}\right)
$$

lifts to an isomorphism

$$
f_{*}: T^{p}(A) \stackrel{\cong}{\longrightarrow} T^{p}\left(A^{\prime}\right) .
$$

The pair of pullback squares (the righthand square is a pullback by Corollary 3.5.3)

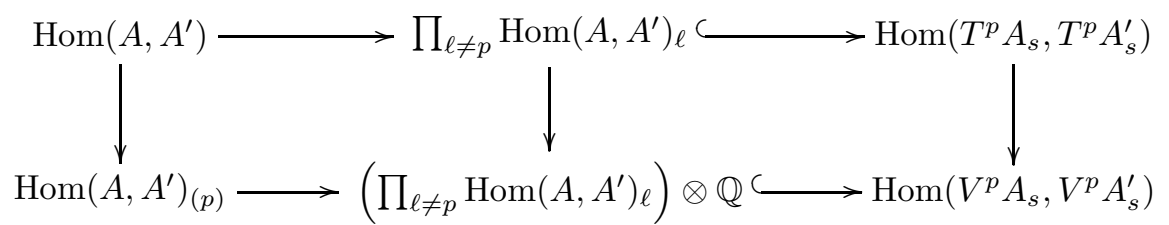

allows us to conclude that $f$ is actually an isomorphism of abelian varieties.

\subsection{Moduli problems with level structure}

Let $K^{p}$ be an open subgroup of $K_{0}^{p}$. Associated to $K^{p}$ are variants of the functors $\mathcal{X}$ and $\mathcal{X}^{\prime}$.

For a locally noetherian connected scheme $S$ on which $p$ is locally nilpotent, with geometric point $s \rightarrow S$, define a groupoid $\mathcal{X}_{K^{p}}(S)$ to have objects $\left(A, i, \lambda,[\eta]_{K^{p}}\right)$ where:

$(A, i, \lambda)$ is an object of $\mathcal{X}(S)$,

$[\eta]_{K^{p}} \quad$ is an integral $K^{p}$ level structure, i.e. the $K^{p}$-orbit of an integral uniformization $\eta: L^{p} \stackrel{\cong}{\longrightarrow} T^{p}\left(A_{s}\right)$, such that $\eta$ is $\mathcal{O}_{B^{-}}$-linear, $[\eta]_{K^{p}}$ is $\pi_{1}(S, s)$-invariant, and $\eta$ sends $\langle-,-\rangle$ to an $\left(\mathbb{A}^{p, \infty}\right)^{\times}$-multiple of the $\lambda$-Weil pairing. 
The morphisms of $\mathcal{X}_{K^{p}}(S)$ are those isomorphisms in $\mathcal{X}(S)$ which preserve the level structure.

Define a groupoid $\mathcal{X}^{\prime} K^{p}(S)$ to have objects $\left(A, i, \lambda,[\eta]_{K^{p}}\right)$ where:

$$
\begin{aligned}
\left(A, i, \lambda,[\eta]_{K_{0}^{p}}\right) \quad \text { is an object of } \mathcal{X}^{\prime}(S), & \\
& \text { is a rational } K^{p} \text { level structure, i.e. the } K^{p} \text {-orbit of a } \\
& \text { rational uniformization } \eta: V_{K^{p}} \cong V^{p}\left(A_{s}\right) \text {, such that } \eta \text { is } \\
& B \text {-linear, }[\eta]_{K^{p}} \text { is } \pi_{1}(S, s) \text {-invariant, and } \eta \text { sends }\langle-,-\rangle \text { to } \\
& \text { an }\left(\mathbb{A}^{p, \infty}\right)^{\times} \text {-multiple of the } \lambda \text {-Weil pairing. }
\end{aligned}
$$

The morphisms of $\mathcal{X}^{\prime}{ }_{K^{p}}(S)$ are those prime-to-p isogenies in $\mathcal{X}^{\prime}(S)$ which preserve the $K^{p}$ level structures.

Clearly, the functors $\mathcal{X}$ and $\mathcal{X}^{\prime}$ are recovered by taking $K^{p}=K_{0}^{p}$.

Since any integral level structure determines a rational level structure, the functors $F_{S}$ lift to give functors

$$
F_{K^{p}, S}: \mathcal{X}_{K^{p}}(S) \rightarrow \mathcal{X}_{K^{p}}^{\prime}(S)
$$

which are natural in $S$. Theorem 6.4.1 generalizes to the following theorem.

TheOREM 6.5.1. The functors $F_{K^{p}, S}$ are equivalences of categories.

REMARK 6.5.2. The functor $\mathcal{X}_{K^{p}}^{\prime}$ has the advantage that it may be defined for any compact open subgroup $K^{p}$ of $G U\left(\mathbb{A}^{p, \infty}\right)$, not just those contained in $K_{0}^{p}$.

\subsection{Shimura stacks}

If $K^{\prime p}<K^{p}$ are open compact subgroups of $G U\left(\mathbb{A}^{p, \infty}\right)$, then there are natural transformations

$$
f_{K^{\prime p}, K^{p}}: \mathcal{X}_{K^{\prime p}}^{\prime}(-) \rightarrow \mathcal{X}_{K^{p}}^{\prime}(-)
$$

given by sending a tuple $\left(A, i, \lambda,[\eta]_{K^{\prime p}}\right)$ to the tuple $\left(A, i, \lambda,[\eta]_{K^{p}}\right)$.

The functors $\mathcal{X}^{\prime}{ }_{K}^{p}$ are representable. For $K^{p}$ sufficiently small, the representability of $\mathcal{X}_{K^{p}}^{\prime}$ by a scheme is discussed in [Kot92, Sec. 5,6]. For general subgroups $K^{p}$, the representability of $\mathcal{X}_{K^{p}}^{\prime}$ by an algebraic stack is discussed in [Hid04, Sec. 7.1.2]. The following theorem represents a compilation of these results.

\section{THEOREM 6.6.2.}

(1) The functor $\mathcal{X}^{\prime}{ }_{K^{p}}$ is representable by a Deligne-Mumford stack $\operatorname{Sh}\left(K^{p}\right)$ over $\mathbb{Z}_{p}$.

(2) The map

$$
f_{K^{\prime p}, K^{p}}: \operatorname{Sh}\left(K^{\prime p}\right) \rightarrow \operatorname{Sh}\left(K^{p}\right)
$$

induced by the natural transformation (6.6.1) is étale, of degree equal to $\left[K^{p}: K^{\prime p}\right]$. If $K^{\prime p}$ is normal in $K^{p}$, then the covering is Galois with Galois group $K^{p} / K^{\prime p}$.

(3) For $K^{p}$ sufficiently small, $\operatorname{Sh}\left(K^{p}\right)$ is a quasi-projective scheme over $\mathbb{Z}_{p}$. It is projective if $B$ is a division algebra. 
REMARK 6.6.3. The moduli problem considered in either of these sources has a slightly different formulation than presented here, due to the fact that the authors of [Kot92], [Hid04] are working over $\mathcal{O}_{F,(u)}$. Specializing to $\mathcal{O}_{F, u}=\mathbb{Z}_{p}$ produces an equivalent moduli problem (see Remark 9.1.2). 



\section{CHAPTER 7}

\section{Deformation theory}

\subsection{Deformations of $p$-divisible groups}

We briefly summarize the deformation theory of 1-dimensional $p$-divisible groups, restricting to the case when the dimension of the formal group is 1, following [HT01, II.1], [Dri74, Prop. 4.5].

Let $k$ be a field of characteristic $p$. Fix a $p$-divisible group $\overline{\mathbb{G}}$ over $k$. Consider the functor

$$
\left.\operatorname{Def}_{\overline{\mathbb{G}}}:\{\text { noetherian complete local rings }\} \rightarrow \text { groupoids }\right\}
$$

whose $R$-objects are given by

$$
\operatorname{Def}_{\overline{\mathbb{G}}}(R)=\left\{\begin{array}{ll} 
& i: k \rightarrow R / \mathfrak{m}_{R}, \\
(\mathbb{G}, i, \alpha): \quad & \mathbb{G} \text { a } p \text {-divisible over } R, \\
& \alpha:\left.\mathbb{G}\right|_{\operatorname{Spec}\left(R / \mathfrak{m}_{R}\right)} \stackrel{\cong}{\longrightarrow} i^{*} \overline{\mathbb{G}}
\end{array}\right\},
$$

and whose $R$-morphisms are those isomorphisms of $p$-divisible groups that restrict to the identity on $\overline{\mathbb{G}}$.

Lubin and Tate showed that deformations of 1-dimensional formal groups of finite height have no automorphisms, and that this functor is represented by a formal scheme.

THeorem 7.1.1 (Lubin-Tate [LT66]). Suppose that $\overline{\mathbb{G}}$ is a 1-dimensional formal group of finite height $h$. The functor $\operatorname{Def}_{\overline{\mathbb{G}}}(-)$ is representable by the formal scheme $\operatorname{Def}_{\overline{\mathbb{G}}}=\operatorname{Spf}(B)$ where $B=W(k)\left[\left[u_{1}, \ldots, u_{h-1}\right]\right]$.

Given a $p$-divisible group $\mathbb{G}=\lim \mathbb{G}_{i}$ over $R$, it is convenient to consider its associated sheaf of $\mathbb{Z}_{p}$-modules on $\operatorname{Spf}(R)$ in the fppf topology. Given a local $R$-algebra $T$, the sections are given by

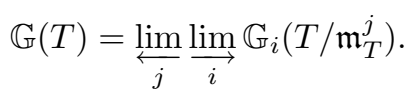

The resulting functor

$$
\{p \text {-divisible groups }\} \rightarrow\left\{\text { fppf sheaves of } \mathbb{Z}_{p} \text {-modules }\right\}
$$

is fully faithful.

Lemma 7.1.2. Suppose $\mathbb{G}$ is a 1-dimensional formal group over $R$ of finite height, with coordinate $x$, so that with respect to this coordinate the sum formula is given 
by

$$
x_{1}+{ }_{\mathbb{G}} x_{2}=\sum_{i, j} a_{i, j} x_{1}^{i} x_{2}^{j}
$$

and that $T$ is a local R-algebra. Then there is an isomorphism of groups

$$
\mathbb{G}(T) \cong\left(\mathfrak{m}_{T},+_{\mathbb{G}}\right) .
$$

Proof. We have an isomorphism of topological rings (see Example 1.1.3):

$$
T[[x]] \cong \lim _{i} T[[x]] /\left[p^{i}\right]_{\mathbb{G}}(x) .
$$

The $T$-sections of $\mathbb{G}$ are then given by the formula

$$
\begin{aligned}
\mathbb{G}(T) & ={\underset{\lim }{j}}_{i} \lim _{i} \operatorname{Alg}_{T}\left(T[[x]] /\left[p^{i}\right]_{\mathbb{G}}(x), T / \mathfrak{m}_{T}^{j}\right) \\
& =\operatorname{Alg}_{T}^{c}(T[[x]], T) \\
& \cong \mathfrak{m}_{T} .
\end{aligned}
$$

We are now in a position to give a description of the deformation theory of 1dimensional $p$-divisible groups.

THEOREM 7.1.3. Suppose that the $\overline{\mathbb{G}}$ is a 1-dimensional $p$-divisible group over $k$ of finite height $h$. Then the functor $\operatorname{Def}_{\overline{\mathbb{G}}}(-)$ is representable by the formal scheme $\operatorname{Def}_{\overline{\mathbb{G}}}=\operatorname{Spf}(B)$, where

$$
B=W(k)\left[\left[u_{1}, \ldots, u_{h-1}\right]\right]
$$

Sketch Proof of Theorem 7.1.3. Let $(\mathbb{G}, i, \alpha)$ be an object of $\operatorname{Def}_{\overline{\mathbb{G}}}(T)$. Then there is a short exact sequence ([HT01, Cor. II.1.2])

$$
0 \rightarrow \mathbb{G}^{0} \rightarrow \mathbb{G} \rightarrow \mathbb{G}^{e t} \rightarrow 0
$$

where $\mathbb{G}^{0}$ is formal of height $k$ and $\mathbb{G}^{e t}$ is ind-étale of height $h-k$. By Theorem 7.1.1, the deformation $\mathbb{G}^{0}$ is classified by a map of local rings

$$
W(k)\left[\left[u_{1}, \ldots, u_{k-1}\right]\right] \rightarrow T .
$$

The deformation $\mathbb{G}^{e t}$ is unique up to isomorphism. The extension is classified by an element of

$$
\operatorname{Ext}_{\mathbb{Z}_{p}}^{1}\left(\mathbb{G}^{e t}, \mathbb{G}^{0}\right)
$$

where the Ext group is taken in the category of sheaves of $\mathbb{Z}_{p}$-modules. Let $T \mathbb{G}^{e t}$ denote the sheaf given by $\lim \mathbb{G}^{e t}\left[p^{i}\right]$. The short exact sequence of sheaves

$$
0 \rightarrow T \mathbb{G}^{e t} \rightarrow T \mathbb{G}^{e t} \otimes_{\mathbb{Z}_{p}} \mathbb{Q}_{p} \rightarrow \mathbb{G}^{e t} \rightarrow 0
$$

gives rise to a long exact sequence of Ext groups. By descent, we may assume that $T$ is separably closed, so that $\mathbb{G}^{e t}$ is abstractly isomorphic to $\left(\mathbb{Q}_{p} / \mathbb{Z}_{p}\right)_{T}^{h-k}$. The boundary homomorphism gives an isomorphism

$$
\operatorname{Hom}_{\mathbb{Z}_{p}}\left(\left(\mathbb{Z}_{p}\right)_{T}^{h-k}, \mathbb{G}^{0}\right) \stackrel{\cong}{\longrightarrow} \operatorname{Ext}_{\mathbb{Z}_{p}}^{1}\left(\mathbb{G}^{e t}, \mathbb{G}^{0}\right) .
$$


Therefore we have,

$$
\begin{aligned}
\operatorname{Def}_{\overline{\mathbb{G}}}(T) & \cong \operatorname{Def}_{\overline{\mathbb{G}}^{0}}(T) \times \operatorname{Hom}_{\mathbb{Z}_{p}}\left(\mathbb{G}^{e t}, \mathbb{G}^{0}\right) \\
& \cong \operatorname{Def}_{\overline{\mathbb{G}}^{0}}(T) \times \operatorname{Hom}_{\mathbb{Z}_{p}}\left(\left(\mathbb{Z}_{p}\right)_{T}^{h-k}, \mathbb{G}^{0}\right) \\
& \cong \operatorname{Def}_{\overline{\mathbb{G}}^{0}}(T) \times \mathfrak{m}_{T}^{h-k}
\end{aligned}
$$

The last isomorphism is given by Lemma 7.1.2. We deduce that there is an isomorphism

$$
\operatorname{Def}_{\bar{G}}(T) \cong \operatorname{Ring}^{c}\left(W(k)\left[\left[u_{1}, \ldots, u_{k-1}\right]\right]\left[\left[u_{k}, \ldots, u_{h-1}\right]\right], T\right) .
$$

\subsection{Serre-Tate theory}

We recall the Serre-Tate theorem [Kat81].

Theorem 7.2.1 (Serre-Tate). Suppose $j: S \hookrightarrow R$ is a closed embedding of schemes with nilpotent ideal sheaf $\mathcal{I}$ such that $p$ is locally nilpotent on $R$. Then the diagram of categories

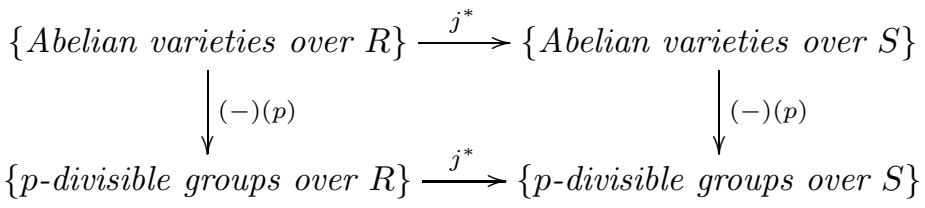

is a pullback. In other words, there is an equivalence of categories between the category of abelian varieties over $R$ and the category of tuples

$$
\left\{\begin{array}{l|l}
(A, \mathbb{G}, \phi) & \begin{array}{l}
A \text { an abelian variety over } S, \\
\mathbb{G} \text { a } p \text {-divisible group over } R, \\
\phi: A\left[p^{\infty}\right] \rightarrow j^{*} \mathbb{G} \text { an isomorphism }
\end{array}
\end{array}\right\} .
$$

\subsection{Deformation theory of points of $S h$}

Let $K^{p}$ be a compact open subgroup of $G U\left(\mathbb{A}^{p, \infty}\right)$. Consider the functor

$$
\begin{aligned}
\Phi: \mathcal{X}_{K^{p}}(S) & \rightarrow p \text {-divisible groups } / S \\
(A, i, \lambda,[\eta]) & \mapsto \epsilon A(u)
\end{aligned}
$$

where $\epsilon A(u)$ is the 1-dimensional summand of the $p$-divisible group $A(p)$. In this section we explain how the deformation theory of points of $\operatorname{Sh}\left(K^{p}\right)$ is controlled by the deformation theory of the associated 1-dimensional $p$-divisible groups. The material in this section is well known (see, for instance, [HT01, Lem. III.4.1]).

TheOREM 7.3.1. Suppose $j: S \hookrightarrow R$ is a closed embedding of schemes with nilpotent ideal sheaf $\mathcal{I}$ such that $p$ is locally nilpotent on $R$. Then the diagram of categories 


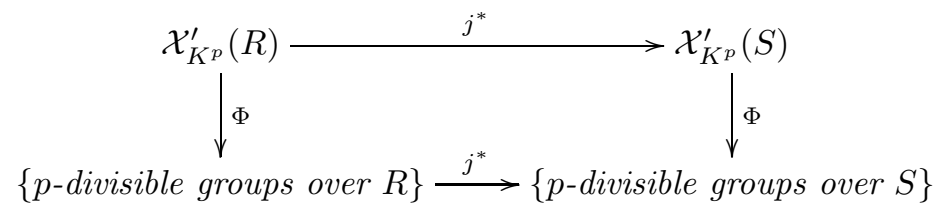

is a pullback. Equivalently, there is an equivalence of categories between the category $\mathcal{X}_{K^{p}}^{\prime}(R)$ and the category of tuples

$$
\left\{\begin{array}{ll} 
& (A, i, \lambda,[\eta]) \text { an object of } \mathcal{X}_{K^{p}}(S), \\
(A, i, \lambda,[\eta], \mathbb{G}, \alpha): \quad & \mathbb{G} \text { a } p \text {-divisible group over } R, \\
& \alpha: \epsilon A(u) \rightarrow j^{*} \mathbb{G} \text { an isomorphism. }
\end{array}\right\}
$$

Proof. The map $i$ takes conjugation on $\mathcal{O}_{F}$ to the Rosati involution. Therefore, the isomorphism

$$
\lambda: A(p) \rightarrow A^{\vee}(p)
$$

breaks up as a direct sum of the following two isomorphisms:

$$
\begin{aligned}
A\left(u^{c}\right) & \rightarrow\left(A^{\vee}\right)(u) \cong A(u)^{\vee}, \\
A(u) & \rightarrow\left(A^{\vee}\right)\left(u^{c}\right) \cong A\left(u^{c}\right)^{\vee} .
\end{aligned}
$$

In particular, $A(u)$ and $A\left(u^{c}\right)$ have the same height with opposite slope decompositions.

The splitting $\mathcal{O}_{B, u} \cong M_{n}\left(\mathbb{Z}_{p}\right)$ gives rise to a further decomposition

$$
A(u) \cong(\epsilon A(u))^{n},
$$

viewed as column vectors acted on by the matrix ring.

The summand $\epsilon A(u)$ of $A(p)$ is 1-dimensional by assumption. The associated formal group $A(p)^{0}$ has a corresponding canonical 1-dimensional summand $\epsilon A(u)^{0}$ of height less than or equal to $n$.

A deformation of $(A, \lambda, i,[\eta])$ to $R$ consists of element $(\tilde{A}, \tilde{\lambda}, \tilde{i},[\tilde{\eta}]) \in \mathcal{X}_{K^{p}}^{\prime}(R)$ and an isomorphism $\phi: A \rightarrow j^{*} \tilde{A}$ respecting the structure. This determines a deformation $\epsilon \tilde{A}(u)$ of the $p$-divisible group $\epsilon A(u)$.

The Serre-Tate theorem provides a functorial equivalence between deformations of $(A, \lambda, i,[\eta])$ and deformations of the associated 1-dimensional summand of the $p$-divisible group, as follows.

Let $\mathbb{G}$ be a deformation of $\epsilon A(u)$ to $R$ with isomorphism $\psi: \epsilon A(u) \rightarrow j^{*} \mathbb{G}$. The isomorphism

$$
\psi^{n}: A(u) \cong(\epsilon A(u))^{n} \rightarrow j^{*}\left(\mathbb{G}^{n}\right)
$$

makes $\mathbb{G}^{n}$ into a deformation of $A(u)$. The action of $\left(\mathcal{O}_{B}\right)_{u} \cong M_{n}\left(\mathbb{Z}_{p}\right)$ extends uniquely to an action on $\mathbb{G}^{n}$.

The dual $p$-divisible group $\left(\mathbb{G}^{\vee}\right)^{n}$ is a deformation of $A\left(u^{c}\right) \cong A(u)^{\vee}$. It carries an action of $\mathcal{O}_{B, u^{c}}$. We obtain an isomorphism

$$
\phi: A(p) \cong A(u) \times A\left(u^{c}\right) \rightarrow j^{*}\left(\mathbb{G}^{n} \times\left(\mathbb{G}^{\vee}\right)^{n}\right)
$$


respecting the action of $\mathcal{O}_{B,(p)}$.

The Serre-Tate theorem then implies that for each deformation, there is an abelian variety $\tilde{A}$ together with isomorphisms

$$
\begin{aligned}
f: j^{*}(\tilde{A}) & \rightarrow A, \\
g: \tilde{A}(p) & \rightarrow \mathbb{G}^{n} \times\left(\mathbb{G}^{\vee}\right)^{n}
\end{aligned}
$$

such that $\phi \circ(f)(p)=j^{*} g$, and the action of $\mathcal{O}_{B,(p)}$ extends to a map

$$
\tilde{i}: \mathcal{O}_{B,(p)} \rightarrow \operatorname{End}(\tilde{A})_{(p)} .
$$

The twist isomorphism

$$
\tau: \mathbb{G}^{n} \times\left(\mathbb{G}^{\vee}\right)^{n} \rightarrow\left(\mathbb{G}^{\vee}\right)^{n} \times \mathbb{G}^{n}
$$

is symmetric, and extends the isomorphism

$$
A(u) \times A\left(u^{c}\right) \rightarrow A^{\vee}(u) \times A^{\vee}\left(u^{c}\right) .
$$

The abelian variety $\tilde{A}^{\vee}$ provides a lifting of the data $\left(A^{\vee},\left(\mathbb{G}^{\vee}\right)^{n} \times \mathbb{G}^{n},\left(\phi^{\vee}\right)^{-1}\right)$. The polarization $\lambda$ on $A$ and the twist morphism $\tau$ lift uniquely to a map $\tilde{\lambda}: \tilde{A} \rightarrow \tilde{A}^{\vee}$. As both $\lambda$ and $\tau$ are symmetric, so is $\tilde{\lambda}$. The maps $\lambda$ and $\tau$ both $*$-commute with the action of $\mathcal{O}_{B,(p)}$, and hence so does $\tilde{\lambda}$.

The positivity of $\tilde{\lambda}$ is characterized by positivity at the geometric points $s$ of $R$, and there is a bijective correspondence between geometric points of $S$ and $R$; therefore, $\tilde{\lambda}$ is positive. Similarly, any level structure $[\eta]$ is determined by its values on the geometric points $s \in S$, and so $[\eta]$ extends uniquely.

Therefore, the extension $\mathbb{G}$ determines a unique deformation $(\tilde{A}, \tilde{\lambda}, \tilde{i},[\eta])$ of the tuple $(A, \lambda, i,[\eta])$ to $R$.

This extension is functorial, as follows. Let $f:(A, \lambda, i,[\eta]) \rightarrow\left(A^{\prime}, \lambda^{\prime}, i^{\prime},\left[\eta^{\prime}\right]\right)$ be a morphism in $\mathcal{X}_{K^{p}}^{\prime}(S)$, and $(\mathbb{G}, \psi),\left(\mathbb{G}^{\prime}, \psi^{\prime}\right)$ corresponding deformations with a choice of extension $f: \mathbb{G} \rightarrow \mathbb{G}^{\prime}$.

The deformation $\mathbb{G}^{n} \times\left(\mathbb{G}^{\vee}\right)^{n}$ of $A(p)$, together with its action of $\mathcal{O}_{B,(p)}$ and twist morphism, is functorial in $\mathbb{G}$. In other words, if $f: A \rightarrow A^{\prime}$ is an honest isogeny of abelian varieties, we have a morphism of Serre-Tate data

$$
\left(A, \mathbb{G}^{n} \times\left(\mathbb{G}^{\vee}\right)^{n}, \phi\right) \rightarrow\left(A^{\prime},\left(\mathbb{G}^{\prime}\right)^{n} \times\left(\left(\mathbb{G}^{\prime}\right)^{\vee}\right)^{n}, \phi^{\prime}\right) .
$$

There is a corresponding map of liftings $\tilde{A} \rightarrow \tilde{A}^{\prime}$. If $f: A \rightarrow A^{\prime}$ is instead a quasi-isogeny, there exists a diagram of isogenies

$$
A \stackrel{[n]}{\longleftarrow} A \stackrel{n f}{\longrightarrow} A^{\prime},
$$

where $n$ is relatively prime to $p$, and hence a diagram of lifts

$$
\tilde{A} \stackrel{[n]}{\longleftarrow} \tilde{A} \stackrel{n \tilde{f}}{\longrightarrow} \tilde{A}^{\prime} .
$$

Therefore, the category of elements of $\mathcal{X}_{K^{p}}^{\prime}(S)$ together with deformations of their $p$-divisible group to $R$ is equivalent to the category $\mathcal{X}^{\prime}(R)$. 
Corollary 7.3.2. Assume that $K^{p}$ is sufficiently small so that $S h\left(K^{p}\right)$ is a scheme. Let $s$ be a point of $S h\left(K^{p}\right)_{p}^{\wedge}$, classifying the tuple $(A, i, \lambda,[\eta])$. Then the map

$$
\Phi: \operatorname{Sh}\left(K^{p}\right)_{s}^{\wedge} \rightarrow \operatorname{Def}_{\epsilon A(u)}
$$

is an isomorphism of formal schemes.

The infinitesimal criterion of smoothness [Gro67, Prop. 17.14.2] allows us to deduce the following.

COROLlary 7.3.3. $\operatorname{Sh}\left(K^{p}\right)$ is smooth of relative dimension $n-1$ over $\operatorname{Spec}\left(\mathbb{Z}_{p}\right)$. 


\section{CHAPTER 8}

\section{Topological automorphic forms}

\subsection{The generalized Hopkins-Miller theorem}

The following terminology is introduced in [Lur].

Definition 8.1.1. A homotopy commutative ring spectrum $E$ is weakly even periodic if

(1) The homotopy groups $\pi_{*} E$ are concentrated in even degrees.

(2) The maps

$$
E^{2} \otimes_{E^{0}} E^{n} \rightarrow E^{n+2}
$$

are isomorphisms.

Let $E$ be a weakly even periodic ring spectrum. The usual $H$-space structure on $\mathbb{C} P^{\infty}$ gives $\mathbb{G}_{E}=\operatorname{Spf}\left(E^{0}\left(\mathbb{C} P^{\infty}\right)\right)$ the structure of a group object in the category of formal schemes. Here, Spf is taken by regarding $E^{0}\left(\mathbb{C} P^{\infty}\right)$ as $\varliminf_{n} E^{0}\left(\mathbb{C} P^{n}\right)$ (condition (1) above implies that there is no $\varliminf^{1}$-term).

Lemma 8.1.2. The formal scheme $\mathbb{G}_{E}$ is a formal group over $\operatorname{Spec}\left(E^{0}\right)$, i.e. it admits the structure of a formal group law Zariski locally over $\operatorname{Spec}\left(E^{0}\right)$.

Proof. Condition (2) of Definition 8.1.1 implies that $E^{2}$ is an invertible $E^{0}$ module with inverse $E^{-2}$. Therefore, $E^{2}$ is locally free (see [Eis95, Thm 11.6(a)], where the noetherian hypothesis is not used). Let $\operatorname{Spec}(R) \subseteq \operatorname{Spec}\left(E^{0}\right)$ be an affine Zariski open so that $R \otimes_{E^{0}} E^{-2} \cong R\{u\}$ is a free $R$-module of rank 1 . Then there are isomorphisms

$$
R \otimes_{E^{0}} E^{*}\left(\mathbb{C} P^{\infty}\right) \cong R \otimes_{E^{0}} E^{*}[[x]] \cong E^{*}[[x u]]
$$

where $x$ lies in degree 2 . The coordinate $x u$ gives $R \otimes_{E^{0}} E^{0}\left(\mathbb{C} P^{\infty}\right)$ the structure of a formal group law.

Let

$$
\omega_{\mathbb{G}_{E}}=\left(\operatorname{Lie}\left(\mathbb{G}_{E}\right)\right)^{*}
$$

be the line bundle over $\operatorname{Spec}\left(E^{0}\right)$ of invariant 1-forms on $\mathbb{G}_{E}$. There is a canonical isomorphism

$$
\pi_{2 t} E \cong \Gamma \omega_{\mathbb{G}_{E}}^{\otimes t} .
$$

Goerss and Hopkins [GH04] extended work of Hopkins and Miller [Rez98] to provide a partial converse to this construction. 
THEOREM 8.1.3 (Goerss-Hopkins-Miller). Let $k$ be a perfect field of characteristic $p$ and let $\overline{\mathbb{G}}$ be a formal group of finite height over $k$. Then there is an even periodic $E_{\infty}$-ring spectrum $E_{\overline{\mathbb{G}}}$ such that

(1) $E_{\overline{\mathbb{G}}}^{0}=\mathcal{O}_{\text {Def }_{\bar{G}}}$, the coordinate ring of the formal scheme $\operatorname{Def}_{\overline{\mathbb{G}}}$.

(2) $\mathbb{G}_{E}$ is a universal deformation of $\overline{\mathbb{G}}$.

The construction is functorial in pairs $(k, \overline{\mathbb{G}})$ : given another pair $\left(k^{\prime}, \overline{\mathbb{G}}^{\prime}\right)$, a map of fields $i: k^{\prime} \rightarrow k$, and an isomorphism $\alpha: \overline{\mathbb{G}} \cong i^{*} \overline{\mathbb{G}}^{\prime}$, there is an induced map of $E_{\infty}$-ring spectra

$$
(i, \alpha)^{*}: E_{\overline{\mathbb{G}}^{\prime}} \rightarrow E_{\overline{\mathbb{G}}}
$$

Our goal in this section is to state a generalization, recently announced by Lurie, which gives functorial liftings of rings with 1-dimensional $p$-divisible groups.

TheOREM 8.1.4 (Lurie). Let $A$ be a local ring with maximal ideal $\mathfrak{m}_{A}$ and residue field $k$ perfect of characteristic $p$, and suppose $X$ is a locally noetherian separated Deligne-Mumford stack over $\operatorname{Spec}(A)$. Suppose that $\mathbb{G} \rightarrow X$ is a p-divisible group over $X$ of constant height $h$ and dimension 1 . Suppose that for some étale cover $\pi: \widetilde{X} \rightarrow X$ where $\widetilde{X}$ is a scheme, the following condition is satisfied:

(*): for every point $x \in \widetilde{X}_{\mathfrak{m}_{A}}^{\wedge}$ the induced map

$$
\tilde{X}_{x}^{\wedge} \rightarrow \operatorname{Def}_{\pi^{*} \mathbb{G}_{x}}
$$

classifying the deformation $\left.\left(\pi^{*} \mathbb{G}\right)\right|_{\tilde{X}_{x}^{\wedge}}$ of $\left(\pi^{*} \mathbb{G}\right)_{x}$ is an isomorphism of formal schemes.

Then there exists a presheaf of $E_{\infty}$-ring spectra $\mathcal{E}_{\mathbb{G}}$ on the étale site of $X_{\mathfrak{m}_{A}}^{\wedge}$, such that

(1) $\mathcal{E}_{\mathbb{G}}$ satisfies homotopy descent: it is (locally) fibrant in the Jardine model structure [Jar00], [DHI04].

(2) For every formal affine étale open $f: \operatorname{Spf}(R) \rightarrow X_{\mathfrak{m}_{A}}^{\wedge}$, the $E_{\infty}$-ring spectrum of sections $\mathcal{E}_{\mathbb{G}}(R)$ is weakly even periodic, with $\mathcal{E}_{\mathbb{G}}(R)^{0}=R$. There is an isomorphism

$$
\gamma_{f}: f^{*} \mathbb{G}^{0} \stackrel{\cong}{\longrightarrow} \mathbb{G}_{\mathcal{E}_{\mathbb{G}}(R)}
$$

natural in $f$.

The construction is functorial in $(X, \mathbb{G})$ : given another pair $\left(X^{\prime}, \mathbb{G}^{\prime}\right)$, a morphism $g: X \rightarrow X^{\prime}$ of Deligne-Mumford stacks over $\operatorname{Spec}(A)$, and an isomorphism of $p$ divisible groups $\alpha: \mathbb{G} \cong g^{*} \mathbb{G}^{\prime}$, there is an induced map of presheaves of $E_{\infty}$-ring spectra

$$
(g, \alpha)^{*}: g^{*} \mathcal{E}_{\mathbb{G}^{\prime}} \rightarrow \mathcal{E}_{\mathbb{G}} .
$$

Remark 8.1.5. Suppose that $\mathbb{G}$ and $X$ satisfy the hypotheses of Theorem 8.1.4. The functoriality of the sections of the presheaf $\mathcal{E}_{\mathbb{G}}$ is subsumed by the functoriality of the presheaf in $\mathbb{G}$. Indeed, suppose that $g: U \rightarrow X_{\mathfrak{M}_{A}}^{\wedge}$ is an étale open. 
(1) The pullback $g^{*} \mathbb{G}$ satisfies condition $(*)$ of Theorem 8.1.4, and hence gives rise to a presheaf $\mathcal{E}_{g^{*} \mathbb{G}}$ on $U$.

(2) The spectrum of sections $\mathcal{E}_{\mathbb{G}}(U)$ is given by the global sections $\mathcal{E}_{g^{*} \mathbb{G}}(U)$.

(3) The map

$$
(g, \mathrm{Id})^{*}: g^{*} \mathcal{E}_{\mathbb{G}} \rightarrow \mathcal{E}_{g^{*} \mathbb{G}}
$$

induces a map

$$
g^{*}: \mathcal{E}_{\mathbb{G}}(X) \rightarrow \mathcal{E}_{g^{*} \mathbb{G}}(U)=\mathcal{E}_{\mathbb{G}}(U)
$$

which agrees with the restriction map for the presheaf $\mathcal{E}_{\mathbb{G}}$.

Suppose that

$$
f: \operatorname{Spf}(R) \rightarrow X_{\mathfrak{m}_{A}}^{\wedge}
$$

is a formal affine étale open, and suppose that the invertible sheaf $f^{*} \omega_{\mathbb{G}^{0}}$ is trivial. By Condition (2) above, this is equivalent to asserting that the spectrum of sections $\mathcal{E}_{\mathbb{G}}(R)$ is even periodic. Then Condition (2) actually determines the homotopy type of the spectrum $\mathcal{E}_{\mathbb{G}}(R)$. Indeed, by choosing a coordinate $T$ of the formal group $f^{*} \mathbb{G}^{0}$ over $\operatorname{Spf}(R)$, we get a map of rings

$$
\rho_{f * \mathbb{G}^{0}, T}: M U P^{0} \rightarrow R=\mathcal{E}_{\mathbb{G}}(R)^{0}
$$

classifying the pair $\left(f^{*} \mathbb{G}^{0}, T\right)$. Here, $M U P$ is the periodic complex cobordism spectrum [Str99, Ex. 8.5]. The ring $M U P^{0}$ is the Lazard ring, and there is a canonical map

$$
\operatorname{Spec}\left(M U P^{0}\right) \rightarrow \mathcal{M}_{F G}
$$

where $\mathcal{M}_{F G}$ is the moduli stack of formal groups.

Lemma 8.1.6. The composite

$$
\operatorname{Spec}(R) \stackrel{\rho_{f^{*} \mathbb{G}^{0}, T}}{\longrightarrow} \operatorname{Spec}\left(M U P^{0}\right) \rightarrow \mathcal{M}_{F G}
$$

is flat.

Proof. We check Landweber's criterion [Lan76], [Nau07]. Let $I_{p, n}$ be the ideal $\left(p, v_{1}, \ldots, v_{n-1}\right)$ of $M U P^{0}$. We must show that for each $n$, the map

$$
\cdot v_{n}: R / I_{p, n} \rightarrow R / I_{p, n}
$$

is injective. Suppose that $r$ is an element of $R / I_{p, n}$. Assuming that $r$ is non-zero, we need to verify that $v_{n} r$ is nonzero. Since $f$ is étale and $X$ is locally noetherian, $R$ must be noetherian. Therefore there is a maximal ideal $\mathfrak{m}$ of $R$ so that the image of $r$ in $R_{\mathfrak{m}}^{\wedge} / I_{p, n}$ is non-zero. Let $x$ be the closed point of $\operatorname{Spf}\left(R_{\mathfrak{m}}^{\wedge}\right)$, and consider the map

$$
\phi: \quad \operatorname{Spf}\left(R_{\mathfrak{m}}^{\wedge}\right) \rightarrow \operatorname{Def}_{f^{*} \mathbb{G}_{x}}
$$

classifying the deformation $\left.f^{*} \mathbb{G}\right|_{\operatorname{Spf}\left(R_{\mathrm{m}}\right)}$. Since condition $(*)$ is an étale local condition, the map $\phi$ is an isomorphism of formal schemes, and gives an isomorphism of $M U P^{0}$-algebras

$$
\phi^{*}: W(k(x))\left[\left[u_{1}, \ldots, u_{h-1}\right]\right] \stackrel{\cong}{\rightrightarrows} R_{\mathfrak{m}}^{\wedge} .
$$

Let $h^{\prime} \leq h$ be the height of the formal group $\mathbb{G}_{f(x)}^{0}$. Lemma 6.10 of [Rez98] implies that multiplication by $v_{n}$ is injective on the subalgebra $W(k(x))\left[\left[u_{1}, \ldots, u_{h^{\prime}-1}\right]\right] / I_{p, n}$ 
classifying deformations of the formal group $\mathbb{G}_{f(x)}^{0}$, hence multiplication by $v_{n}$ is injective on

$$
W(k(x))\left[\left[u_{1}, \ldots, u_{h-1}\right]\right] / I_{p, n}=\left(W(k(x))\left[\left[u_{1}, \ldots, u_{h^{\prime}-1}\right]\right] / I_{p, n}\right)\left[\left[u_{h^{\prime}}, \ldots, u_{h-1}\right]\right] .
$$

Thus the image of $v_{n} r$ in this ring is non-zero, implying that $v_{n} r$ must be nonzero.

We conclude that the theories $\mathcal{E}_{\mathbb{G}}(R)$ are Landweber exact [Lan76], [Nau07].

COROLLARY 8.1.7. There is an isomorphism of cohomology theories

$$
R \otimes_{M U P^{0}} M U P^{*}(X) \cong \mathcal{E}_{\mathbb{G}}(R)^{*}(X) .
$$

Remark 8.1.8. Hovey and Strickland [HS99, Prop. 2.20] showed that for Landweber exact cohomology theories such as $\mathcal{E}_{\mathbb{G}}(R)$ which have no odd dimensional homotopy groups, Corollary 8.1.7 actually determines the homotopy type of these spectra.

Corollary 8.1.9. Suppose that $k$ is a perfect field of characteristic $p$ and that $\overline{\mathbb{G}}$ is a formal group of finite height over $k$. Let $\mathbb{G} / \operatorname{Def}_{\overline{\mathbb{G}}}$ be the universal deformation of $\overline{\mathbb{G}}$. Then there is an equivalence

$$
E_{\overline{\mathbb{G}}} \simeq \mathcal{E}_{\mathbb{G}}\left(\operatorname{Def}_{\overline{\mathbb{G}}}\right) .
$$

PROOF. Both spectra are Landweber exact cohomology theories whose associated formal groups are isomorphic.

\subsection{The descent spectral sequence}

Let $X$ and $\mathbb{G}$ be as in Theorem 8.1.4. Let $\omega_{\mathbb{G}^{0}}$ be the line bundle of invariant 1 -forms on the formal subgroup $\mathbb{G}^{0}$. In the previous section we have identified the

homotopy groups of the spectrum of sections of $\mathcal{E}_{\mathbb{G}}(U)$ for formal affine étale opens

$$
f: U \rightarrow X_{\mathfrak{m}_{A}}^{\wedge} .
$$

Namely, for $k$ odd, $\pi_{k}\left(\mathcal{E}_{\mathbb{G}}(R)\right)=0$ whereas

$$
\pi_{2 t}\left(\mathcal{E}_{\mathbb{G}}(U)\right)=H^{0}\left(U, \omega_{f * \mathbb{G}^{0}}^{\otimes t}\right) .
$$

For an arbitrary étale open

$$
f: U \rightarrow X_{\mathfrak{m}_{A}}^{\wedge}
$$

we have the following.

THEOREM 8.2.1. There is a conditionally convergent spectral sequence

$$
E_{2}^{s, 2 t}=H_{z a r}^{s}\left(U, \omega_{f^{*} \mathbb{G}^{0}}^{\otimes t}\right) \Rightarrow \pi_{2 t-s}\left(\mathcal{E}_{\mathbb{G}}(U)\right) .
$$

Proof. Let $U^{\prime} \rightarrow U$ be a formal affine étale open. Consider the cosimplicial object given by the Cech nerve:

$$
\mathcal{E}_{\mathbb{G}}\left(U^{\prime \bullet+1}\right)=\left\{\mathcal{E}_{\mathbb{G}}\left(U^{\prime}\right) \Rightarrow \mathcal{E}_{\mathbb{G}}\left(U^{\prime} \times_{U} U^{\prime}\right) \Rightarrow \cdots\right\}
$$

Because the sheaf $\mathcal{E}_{\mathbb{G}}$ satisfies homotopy descent, the map

$$
\mathcal{E}_{\mathbb{G}}(U) \rightarrow \underset{\Delta}{\operatorname{holim}} \mathcal{E}_{\mathbb{G}}\left(U^{\prime \bullet+1}\right)
$$


is an equivalence. The spectral sequence of the theorem is the Bousfield-Kan spectral sequence for computing the homotopy groups of the homotopy totalization. Since $U^{\prime}$ is an affine formal scheme and $X$ is separated, the pullbacks $U^{\prime} \times_{U} \cdots \times_{U} U^{\prime}$ are affine formal schemes. Therefore, the $E_{2}^{*, 2 t}$-term is the cohomology of the cosimplicial abelian group

$$
\omega_{f * \mathbb{G}}^{\otimes t}\left(U^{\prime}\right) \Rightarrow \omega_{f * \mathbb{G}}^{\otimes t}\left(U^{\prime} \times_{U} U^{\prime}\right) \Rightarrow \cdots
$$

Since the coherent sheaf $\omega_{f^{*} \mathbb{G}}^{\otimes t}$ has no higher cohomology when restricted to $U^{\prime} \times_{U}$ $\cdots \times{ }_{U} U^{\prime}$, we deduce that the cohomology of this cosimplicial abelian group computes the sheaf cohomology of $\omega_{f^{*} \mathbb{G}}^{\otimes t}$ over $U$.

We are able to deduce the following generalization of Corollary 8.1.7.

Proposition 8.2.2. Suppose that

$$
f: U \rightarrow X_{\mathfrak{m}_{A}}^{\wedge}
$$

is an étale open, and that for every quasi-coherent sheaf $\mathcal{F}$ on $U$

$$
H_{\text {zar }}^{s}(U, \mathcal{F})=0
$$

for $s>0$. Then the spectrum $E=\mathcal{E}_{\mathbb{G}}(U)$ is Landweber exact.

Proof. Let $g: U^{\prime} \rightarrow U$ be a formal affine étale cover. By Lemma 8.1.6 the composite

$$
U^{\prime} \stackrel{g}{\rightarrow} U \stackrel{f}{\rightarrow} X_{\mathfrak{m}_{A}}^{\wedge} \rightarrow \mathcal{M}_{F G}
$$

is flat. Since $g$ is faithfully flat, we deduce that the composite

$$
h: U \stackrel{f}{\rightarrow} X_{\mathfrak{m}_{A}}^{\wedge} \rightarrow \mathcal{M}_{F G}
$$

is flat.

By hypothesis the descent spectral sequence of Theorem 8.2.1 collapses to give

$$
\pi_{k}(E) \cong \begin{cases}H^{0}\left(U, \omega^{\otimes t}\right), & k=2 t \\ 0, & k \text { odd }\end{cases}
$$

Since $\pi_{*} E$ is concentrated in even degrees, $E$ is complex orientable. Let $X$ be a spectrum, and let $\mathcal{M U}^{e v}(X)$ (respectively $\mathcal{M U}^{\text {odd }}(X)$ ) be the sheaf over $\mathcal{M}_{F G}$ determined by the $M U_{*} M U$-comodule $M U_{2 *}(X)$ (respectively $M U_{2 *+1}(X)$ ). We have

$$
\left(M U_{*}(X) \otimes_{M U_{*}} E_{*}\right)_{k}= \begin{cases}H^{0}\left(U, h_{\text {coh }}^{*}\left(\mathcal{M U}^{e v} \otimes \omega^{t}\right)\right), & k=2 t, \\ H^{0}\left(U, h_{c o h}^{*}\left(\mathcal{M} \mathcal{U}^{o d d} \otimes \omega^{t}\right)\right), & k=2 t+1 .\end{cases}
$$

Since $h_{c o h}^{*}$ and $H_{c o h}^{0}(U,-)$ are both exact functors, we deduce that $M U_{*}(-) \otimes_{M U_{*}}$ $E_{*}$ is a homology theory, and hence that $E$ is Landweber exact. 


\subsection{Application to Shimura stacks}

Let $S h\left(K^{p}\right)$ be the Shimura stack over $\mathbb{Z}_{p}$ of Chapter 6 . Define a line bundle $\omega$ over $S h\left(K^{p}\right)$ as follows: for an $S$-point of $S h\left(K^{p}\right)$ classifying a tuple $(A, i, \lambda,[\eta])$ over $S$, let

$$
\omega=\omega_{\epsilon A(u)^{0}},
$$

the module of invariant 1-forms on the formal part of the 1-dimensional summand $\epsilon A(u)$ of the $p$-divisible group $A(p)$.

Corollary 7.3.2 implies that the $p$-divisible group $\epsilon A(u)$ on the Shimura stack $\operatorname{Sh}\left(K^{p}\right)$ satisfies condition (*) of Theorem 8.1.4. Thus, there is a presheaf $\mathcal{E}\left(K^{p}\right)$ of $E_{\infty}$-ring spectra on the étale site of $S h\left(K^{p}\right)_{p}^{\wedge}$, satisfying the following.

(1) For a formal affine étale open

$$
f: \operatorname{Spf}(R) \rightarrow \operatorname{Sh}\left(K^{p}\right)_{p}^{\wedge}
$$

classifying the tuple $(A, i, \lambda,[\eta])$ over $\operatorname{Spf}(R)$, the spectrum of sections $\mathcal{E}\left(K^{p}\right)(R)$ is a weakly even periodic $E_{\infty}$-ring spectrum, equipped with an isomorphism

$$
\mathbb{G}_{\mathcal{E}\left(K^{p}\right)(R)} \cong \epsilon A(u)^{0}
$$

of its formal group with the formal subgroup $\epsilon A(u)^{0}$ of $A(p)$.

(2) For an étale open $f: U \rightarrow S h\left(K^{p}\right)_{p}^{\wedge}$, there is a spectral sequence

$$
H^{s}\left(U, \omega^{\otimes t}\right) \Rightarrow \pi_{2 t-s}\left(\mathcal{E}\left(K^{p}\right)(U)\right) .
$$

In particular, we define a spectrum of topological automorphic forms by

$$
T A F\left(K^{p}\right)=\mathcal{E}\left(K^{p}\right)\left(S h\left(K^{p}\right)_{p}^{\wedge}\right),
$$

with descent spectral sequence

$$
H^{s}\left(\operatorname{Sh}\left(K^{p}\right)_{p}^{\wedge}, \omega^{\otimes t}\right) \Rightarrow \pi_{2 t-s}\left(T A F\left(K^{p}\right)\right) .
$$

The following lemma follows immediately from Remark 8.1.5.

Lemma 8.3.2. Let $K^{\prime p}$ be an open subgroup of $K^{p}$. The spectrum $T A F\left(K^{\prime p}\right)$ is given as the sections of the sheaf $\mathcal{E}\left(K^{p}\right)$ on the étale cover

$$
f_{K^{\prime p}, K^{p}}: \operatorname{Sh}\left(K^{\prime p}\right) \rightarrow \operatorname{Sh}\left(K^{p}\right)
$$

of Theorem 6.6.2. 


\section{CHAPTER 9}

\section{Relationship to automorphic forms}

Assume that $K^{p}$ is sufficiently small, so that $S h\left(K^{p}\right)$ is a quasi-projective scheme. We briefly explain the connection with the classical theory of holomorphic automorphic forms. Namely, we will explain that there is a variant $\operatorname{Sh}\left(K^{p}\right)_{F}$ of the Shimura variety defined over $F$, so that there is an isomorphism

$$
\operatorname{Sh}\left(K^{p}\right) \otimes_{\mathcal{O}_{F, u}} F_{u} \cong S h\left(K^{p}\right)_{F} \otimes_{F} F_{u} .
$$

There is therefore a zig-zag of maps

$$
\begin{aligned}
H^{0}\left(\operatorname{Sh}\left(K^{p}\right), \omega^{\otimes k}\right) \longrightarrow & H^{0}\left(\operatorname{Sh}\left(K^{p}\right)_{F_{u}}, \omega^{\otimes k}\right) \\
& H^{0}\left(\operatorname{Sh}\left(K^{p}\right)_{F}, \omega^{\otimes k}\right) \longrightarrow H^{0}\left(\operatorname{Sh}\left(K^{p}\right)_{\mathbb{C}}, \omega^{\otimes k}\right)
\end{aligned}
$$

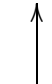

where

$$
\begin{aligned}
S h\left(K^{p}\right)_{F_{u}} & =S h\left(K^{p}\right)_{F} \otimes_{F} F_{u}, \\
S h\left(K^{p}\right)_{\mathbb{C}} & =S h\left(K^{p}\right)_{F} \otimes_{F} \mathbb{C} .
\end{aligned}
$$

We will explain that the sections

$$
H^{0}\left(\operatorname{Sh}\left(K^{p}\right)_{\mathbb{C}}, \omega^{\otimes k}\right)
$$

give instances of classical holomorphic automorphic forms for $G U$.

REMARK 9.0.3. There is a further variant $S h\left(K^{p}\right)_{(u)}$ of the Shimura variety $\operatorname{Sh}\left(K^{p}\right)$ which is defined over the local ring $\mathcal{O}_{F,(u)}$ studied in [Kot92] and [Hid04]. The varieties $S h\left(K^{p}\right)$ and $S h\left(K^{p}\right)_{F}$ are obtained from $S h\left(K^{p}\right)_{(u)}$ by base change to $\mathcal{O}_{F, u}$ and $\mathcal{F}$, respectively (see Remarks 9.1.2 and 9.2.2).

\subsection{Alternate description of $S h\left(K^{p}\right)$}

For an abelian scheme $A$, let Lie $A$ denote the tangent sheaf at the identity section. For a $p$-divisible group $\mathbb{G}$ over a locally $p$-nilpotent base, let Lie $\mathbb{G}$ be the sheaf of invariant vector fields in the formal group $\mathbb{G}^{0}$.

In Section 6.3 we defined a functor

$$
\left.\mathcal{X}_{K^{p}}^{\prime}:\left\{\begin{array}{c}
\text { locally noetherian schemes } \\
\text { on which } p \text { is locally nilpotent }
\end{array}\right\} \rightarrow \text { \{groupoids }\right\} .
$$

The functor of points of the Deligne-Mumford stack $S h\left(K^{p}\right)$ restricts to give the functor $\mathcal{X}_{K^{p}}^{\prime}$. In order to treat the characteristic zero points of the stack, we must 
describe an extension

$$
\mathcal{X}_{K^{p}}^{\prime \prime}:\left\{\text { locally noetherian schemes } / \operatorname{Spec}\left(\mathbb{Z}_{p}\right)\right\} \rightarrow\{\text { groupoids }\}
$$

of the functor $\mathcal{X}_{K^{p}}^{\prime}$.

For a locally noetherian connected scheme $S$, the objects of the groupoid $\mathcal{X}_{K^{p}}^{\prime \prime}$ are tuples $(A, i, \lambda,[\eta])$ just as in the definition of $\mathcal{X}_{K^{p}}^{\prime}$ with one modification: the condition that $\epsilon A(u)$ is 1-dimensional must be replaced with the following condition on Lie $A$ :

(*): The coherent sheaf Lie $A \otimes_{\mathcal{O}_{F, p}} \mathcal{O}_{F, u}$ is locally free of rank $n$.

The tensor product in condition $(*)$ above is taken with respect to the action of $\mathcal{O}_{F, p}$ on Lie $A$ induced from the $B$-linear structure $i$ on $A$.

LEMMA 9.1.1. There is a natural isomorphism of functors

$$
\mathcal{X}_{K^{p}}^{\prime} \cong \mathcal{X}_{K^{p}}^{\prime \prime}
$$

(where $\mathcal{X}_{K^{p}}^{\prime \prime}$ has been restricted to the domain of $\mathcal{X}_{K^{p}}^{\prime}$ ).

Proof. If $S$ is a locally noetherian connected scheme for which $p$ is locally nilpotent, we must show that a tuple $(A, i, \lambda,[\eta])$ is an object of $\mathcal{X}_{K^{p}}^{\prime}(S)$ if and only if it is an object of $\mathcal{X}_{K^{p}}^{\prime \prime}(S)$. However, since $p$ is locally nilpotent on $S$, there is a natural isomorphism

$$
\operatorname{Lie} A(p) \cong \operatorname{Lie} A
$$

which induces a natural isomorphism

$$
\text { Lie } A(u) \cong \operatorname{Lie} A \otimes_{\mathcal{O}_{F, p}} \mathcal{O}_{F, u} .
$$

The coherent sheaf Lie $A(u)$ is locally free of rank $n$ if and only if the summand $\epsilon$ Lie $A(u)$ is locally free of rank 1 . The latter is equivalent to the formal part of the $p$-divisible group $\epsilon A(u)$ having dimension equal to 1 .

REMARK 9.1.2. In [Kot92] and [Hid04], a slightly different functor

$$
\mathcal{X}_{K^{p}}^{\prime \prime \prime}:\left\{\text { locally noetherian schemes/ } \operatorname{Spec}\left(\mathcal{O}_{F,(u)}\right)\right\} \rightarrow \text { \{roupoids }
$$

is studied. The $S$-points are still given by tuples $(A, i, \lambda,[\eta])$, but condition $(*)$ is replaced with a "determinant condition" [Kot92, p390], [Hid04, p308]. When $S$ is a scheme over $\mathcal{O}_{F, u}$, the determinant condition implies that there is a local isomorphism of $\mathcal{O}_{B, p}$-linear $\mathcal{O}_{S}$-modules

$$
\text { Lie } A \cong \mathcal{O}_{F, u}^{n} \otimes_{\mathcal{O}_{F, u}} \mathcal{O}_{S} \oplus\left(\mathcal{O}_{F, u^{c}}^{n}\right)^{n-1} \otimes_{c, \mathcal{O}_{F, u}} \mathcal{O}_{S} .
$$

Here we are implicitly using our fixed isomorphism $\mathcal{O}_{B, p} \cong M_{n}\left(\mathcal{O}_{F, p}\right)$, and $\mathcal{O}_{F, u}^{n}$ (respectively $\mathcal{O}_{F, u^{c}}^{n}$ ) is the standard representation of the algebra $M_{n}\left(\mathcal{O}_{F, u}\right)$ (respectively $\left.M_{n}\left(\mathcal{O}_{F, u^{c}}\right)\right)$. The notation $\otimes_{c, \mathcal{O}_{F, u}}$ above indicates that we are regard-

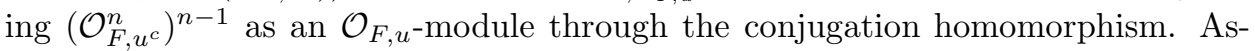
serting the existence of such a local isomorphism is equivalent to asserting that Lie $A \otimes_{\mathcal{O}_{F, p}} \mathcal{O}_{F, u}$ is locally free of rank $n$. The functor $\mathcal{X}_{K^{p}}^{\prime \prime \prime}$ is proven in [Kot92, Sec. 5,6] to be representable by a quasi-projective variety $S h\left(K^{p}\right)_{(u)}$ when $K^{p}$ is sufficiently small. For general $K^{p}$, the representing object $S h\left(K^{p}\right)_{(u)}$ still exists as 
a Deligne-Mumford stack [Hid04, Sec 7.1.2]. The Shimura stack $S h\left(K^{p}\right)$ which represents the functors $\mathcal{X}_{K^{p}}^{\prime}$ and $\mathcal{X}_{K^{p}}^{\prime \prime}$ is obtained from $S h\left(K^{p}\right)_{(u)}$ by defining

$$
\operatorname{Sh}\left(K^{p}\right):=\operatorname{Sh}\left(K^{p}\right)_{(u)} \otimes_{\mathcal{O}_{F,(u)}} \mathcal{O}_{F, u} .
$$

\subsection{Description of $S h\left(K^{p}\right)_{F}$}

We now describe the stack $S h\left(K^{p}\right)_{F}$ over $F$. Let $S$ be a locally noetherian connected scheme over $F$. An object of the groupoid of $S$-points $S h\left(K^{p}\right)_{F}(S)$ consists of a tuple $(A, i, \lambda,[\eta])$ just as in the definition of the functor $\mathcal{X}_{K^{p}}^{\prime}$ except that the condition that $\epsilon A(u)$ is 1-dimensional must be replaced with a suitable condition on the locally free sheaf Lie $A$ on $S$. The field $F$ acts on Lie $A$ in two different ways: both through the complex multiplication $i$ of $F$ on $A$, and through structure of $S$ as a scheme over $F$. This induces a splitting of the sheaf Lie $A$ :

$$
\text { Lie } A \cong \text { Lie } A^{+} \oplus \text { Lie } A^{-}
$$

where $\operatorname{Lie} A^{+}$is the subsheaf where the $F$-linear structures agree, and Lie $A^{-}$is the subsheaf where the $F$-linear structures are conjugate. We require:

(*): the sheaf Lie $A^{+}$must be locally free of rank $n$.

Lemma 9.2.1. There is an equivalence of stacks

$$
S h\left(K^{p}\right) \otimes_{\mathcal{O}_{F, u}} F_{u} \simeq S h\left(K^{p}\right)_{F} \otimes_{F} F_{u} .
$$

Proof. For an $S$-object $(A, i, \lambda,[\eta])$ of either stack, the sheaf Lie $A^{+}$is naturally isomorphic to Lie $A \otimes_{\mathcal{O}_{F, p}} \mathcal{O}_{F, u}$.

REMARK 9.2.2. When working in characteristic 0 , the determinant condition of Remark 9.1.2 can be replaced by a "trace condition". The points of the resulting stack $S h\left(K^{p}\right)_{F}=S h\left(K^{p}\right)_{(u)} \otimes_{\mathcal{O}_{F,(u)}} F$ are precisely those tuples $(A, i, \lambda,[\eta])$ which satisfy condition $(*)$ above (see [HT01, Lemma III.1.2]).

\subsection{Description of $S h\left(K^{p}\right)_{\mathbb{C}}$}

Let $K^{p}$ be sufficiently small so that $S h\left(K^{p}\right)_{F}$ is a scheme. Fix an embedding

$$
j: F \hookrightarrow \mathbb{C} .
$$

We shall explain how the pullback

$$
\operatorname{Sh}\left(K^{p}\right)_{\mathbb{C}}=\operatorname{Sh}\left(K^{p}\right)_{F} \times_{\operatorname{Spec}(F)} \operatorname{Spec}(\mathbb{C})
$$

admits a classical description (Theorem 9.3.5). The data of Section 6.2 allows us to construct explicit points of $S h\left(K^{p}\right)_{\mathbb{C}}$. Let $W$ be the $2 n^{2}$ dimensional real vector space given by $V_{\infty}=V \otimes_{\mathbb{Q}} \mathbb{R}$ with lattice $L$. A complex structure $J$ is an element of $G L(W)$ satisfying $J^{2}=-1$. For each such $J$, the real vector space $W$ admits the structure of a complex vector space, which we denote $W_{J}$. Associated to $J$ is a polarized abelian variety $A_{J}$ given by the quotient

$$
A_{J}=W_{J} / L \text {. }
$$


The dual abelian variety is given by

$$
A_{J}^{\vee}=W_{J}^{*} / L^{*}
$$

where

$$
\begin{aligned}
W_{J}^{*} & =\left\{\alpha: W_{J} \rightarrow \mathbb{C}: \alpha \text { conjugate linear }\right\} \\
L^{*} & =\left\{\alpha \in W_{J}^{*}: \operatorname{Im} \alpha(L) \subseteq \mathbb{Z}\right\} .
\end{aligned}
$$

Associated to the non-degenerate alternating form $\langle-,-\rangle$ on $V_{\infty}$ is a non-degenerate hermitian form $(-,-)_{J}$ on $W_{J}$, given by the correspondence of Lemma 5.1.2:

$$
(v, w)_{J}=\langle J v, w\rangle+i\langle v, w\rangle .
$$

We get an induced complex-linear isomorphism:

$$
\begin{aligned}
\tilde{\lambda}_{J}: W_{J} & \rightarrow W_{J}^{*}, \\
v & \mapsto(v,-)_{J} .
\end{aligned}
$$

The integrality conditions imposed on $\langle-,-\rangle$ with respect to the lattice $L$ (Section 6.2) imply that $\tilde{\lambda}_{J}$ descends to a prime-to- $p$ isogeny of abelian varieties:

$$
\lambda_{J}: A_{J} \rightarrow A_{J}^{\vee} .
$$

In order for the isogeny $\lambda_{J}$ to be a polarization, the alternating form $\langle-,-\rangle$ must be a Riemann form: we require that the form $\langle-, J-\rangle$ be symmetric and positive.

The $B$-action on $V$ gives rise to an embedding

$$
B \hookrightarrow \operatorname{Aut}_{\mathbb{R}}(W) .
$$

In order for $B$ to act by complex linear maps, we must have

$$
J \in C_{\infty} \subset \operatorname{Aut}_{\mathbb{R}}(W) .
$$

Here, $C_{\infty}$ is the algebra $C \otimes_{\mathbb{Q}} \mathbb{R}$, where $C$ is the algebra of Section 6.2. Since $\mathcal{O}_{B}$ acts on $L$, we get an inclusion

$$
i: \mathcal{O}_{B} \hookrightarrow \operatorname{End}\left(A_{J}\right) .
$$

Let $(-)^{\iota}$ be the involution on $C$ of Section 6.2. Then in order for the involution $*$ on $B$ to agree with the $\lambda_{J}$-Rosati involution, we must have $J^{\iota}=-J$.

The $B$-linear polarized abelian variety $\left(A_{J}, i, \lambda_{J}\right)$ admits a canonical integral uniformization $\eta_{1}$ by the composite

$$
\begin{aligned}
\eta_{1}: L^{p} & =\lim _{(N, p)=1} L / N L \\
& \cong \lim _{(N, p)=1} N^{-1} L / L \\
& =\lim _{(N, p)=1} A_{J}[N] \\
& =T^{p}\left(A_{J}\right) .
\end{aligned}
$$

For $g \in G U\left(\mathbb{A}^{p, \infty}\right)$, we let $\eta_{g}$ denote the associated rational uniformization

$$
\eta_{g}: V^{p} \stackrel{g}{\rightarrow} V^{p} \stackrel{\eta_{1}}{\longrightarrow} V^{p}\left(A_{J}\right) .
$$


Let $W_{J}^{+} \subseteq W_{J}$ denote the subspace where the complex structure $J$ agrees with the complex structure $W_{J}$ inherits from the $F$-linear structure of $W$ through our chosen isomorphism $j: F \otimes_{\mathbb{Q}} \mathbb{R} \cong \mathbb{C}$. Then requiring that Lie $A^{+}$is $n$-dimensional amounts to requiring that $W_{J}^{+}$is $n$-dimensional.

Let $\mathcal{H}$ be the set of compatible complex structures:

$$
\mathcal{H}=\left\{\begin{array}{ll}
J \in C_{\infty}: \begin{array}{l}
J^{2}=-1, \\
\langle-, J-\rangle \text { is symmetric and positive. }
\end{array}
\end{array}\right\}
$$

Lemma 9.3.1. Suppose that $J$ is a compatible complex structure. Then we have:

$$
\begin{gathered}
J^{\iota}=-J, \\
\operatorname{dim}_{\mathbb{C}} W_{J}^{+}=n .
\end{gathered}
$$

Proof. Using the fact that $\langle-, J-\rangle$ is symmetric, we have

$$
\begin{aligned}
\langle x, J y\rangle & =\langle y, J x\rangle \\
& =\left\langle J^{\iota} y, x\right\rangle \\
& =-\left\langle x, J^{\iota} y\right\rangle .
\end{aligned}
$$

Since $\langle-,-\rangle$ is non-degenerate, it follows that $J^{\iota}=-J$.

Our assumptions on the alternating form $\langle-,-\rangle$ imply that the associated $*-$ symmetric form

$$
(x, y)=\langle\delta x, y\rangle+\delta\langle x, y\rangle
$$

on $W$ has signature $(n,(n-1) n)$. Here, $F=\mathbb{Q}(\delta)$, where, under our fixed complex embedding, $\delta$ is equal to $-a i$ for $a \in \mathbb{R}_{>0}$. Suppose that $x$ is a non-zero element of $W_{J}^{+}$. Then we have

$$
\begin{aligned}
(x, x) & =\langle\delta x, x\rangle+\delta\langle x, x\rangle \\
& =\langle-a i x, x\rangle \\
& =a\langle x, i x\rangle \\
& =a\langle x, J x\rangle>0,
\end{aligned}
$$

since we have assumed that $\langle-, J-\rangle$ is positive definite. We deduce, using the signature of $(-,-)$, that $\operatorname{dim} W_{J}^{+} \leq n$. However, the same argument shows that $(-,-)$ is negative definite when restricted to $W_{J}^{-}$, which implies that $\operatorname{dim} W_{J}^{-} \leq$ $(n-1) n$. Since

we deduce that $\operatorname{dim} W_{J}^{+}=n$.

$$
\operatorname{dim} W_{J}^{+}+\operatorname{dim} W_{J}^{-}=n^{2},
$$

We deduce the following lemma.

Lemma 9.3.2. For $J \in \mathcal{H}$, the tuple $\left(A_{J}, i, \lambda_{J},\left[\eta_{g}\right]_{K^{p}}\right)$ gives a $\mathbb{C}$-point of $\operatorname{Sh}\left(K^{p}\right)_{\mathbb{C}}$.

The group

$$
G U(\mathbb{R})^{+}=\left\{g \in G U(\mathbb{R}): g^{\iota} g>0\right\}
$$

acts on $\mathcal{H}$ by conjugation. Under the identification $U(\mathbb{R}) \cong U(1, n-1)$ of Section 6.2 , we have the following lemma. 
Lemma 9.3.3. The action of $U(\mathbb{R})$ on $\mathcal{H}$ is transitive, and the stabilizer of some fixed $J_{0} \in \mathcal{H}$ is given by a maximal compact subgroup

$$
K_{\infty}=U\left(W_{J}^{+}\right) \times U\left(W_{J}^{-}\right) \cong U(1) \times U(n-1) .
$$

Thus there is an isomorphism $\mathcal{H} \cong U(\mathbb{R}) / K_{\infty}$.

REMARK 9.3.4. The symmetric hermitian domain $\mathcal{H}=U(\mathbb{R}) / K_{\infty}$ admits a canonical complex structure [Hel78, VIII].

Define a group

$$
G U\left(\mathbb{Z}_{(p)}\right)^{+}=\left\{g \in G U(\mathbb{Q})^{+}: g\left(L_{(p)}\right)=L_{(p)}\right\} .
$$

Every element $\gamma \in G U\left(\mathbb{Z}_{(p)}\right)^{+}$gives rise to a $\mathbb{Z}_{(p)}$-isogeny

$$
\gamma:\left(A_{J}, i, \lambda_{J},\left[\eta_{g}\right]_{K^{p}}\right) \stackrel{\cong}{\leftrightarrows}\left(A_{\gamma J \gamma^{-1}}, i, \lambda_{\gamma J \gamma^{-1}},\left[\eta_{\gamma g}\right]_{K^{p}}\right) .
$$

We have the following theorem [Kot92, Sec. 8].

Theorem 9.3.5. The map

$$
G U\left(\mathbb{Z}_{(p)}\right)^{+} \backslash\left(G U\left(\mathbb{A}^{p, \infty}\right) / K^{p} \times \mathcal{H}\right) \rightarrow S h\left(K^{p}\right)_{\mathbb{C}}
$$

sending a point $[g, J]$ to the point that classifies $\left(A_{J}, i, \lambda_{J},\left[\eta_{g}\right]_{K^{p}}\right)$ is an isomorphism of complex analytic manifolds.

REMARK 9.3.6. The statement of Theorem 9.3.5 differs mildly that of [Kot92], where it is proven that $S h\left(K^{p}\right)_{\mathbb{C}}$ is isomorphic to

$$
\coprod_{\operatorname{ker}^{1}(\mathbb{Q}, G U)} G U(\mathbb{Q})^{+} \backslash\left(G U\left(\mathbb{A}^{\infty}\right) / K^{p} K_{p} \times \mathcal{H}\right)
$$

where

$$
\begin{gathered}
\operatorname{ker}^{1}(\mathbb{Q}, G U)=\operatorname{ker}\left(H^{1}(\mathbb{Q}, G U) \rightarrow \bigoplus_{v} H^{1}\left(\mathbb{Q}_{v}, G U\right)\right), \\
K_{p}=\left\{g \in G U\left(\mathbb{Q}_{p}\right): g\left(L_{p}\right)=L_{p}\right\} .
\end{gathered}
$$

In our case, the ker $^{1}$-term is trivial (Corollary 5.3.7). Moreover, we may remove the $p$-component from the adelic quotient by combining Lemma 7.2 of [Kot92] with the fact that $H^{1}\left(\mathbb{Q}_{p}, G U\right)$ is trivial (Lemma 5.3.1).

\subsection{Automorphic forms}

Let $T \cong\left(S^{1}\right)^{n}$ be the maximal torus of $K_{\infty}=U(1) \times U(n-1)$, and let $X \cong \mathbb{Z}^{n}$ be the lattice of characters of $T$. Let $V_{\kappa}$ be the finite dimensional irreducible complex representation of the group $K_{\infty}$ of highest weight $\kappa \in X$. The collection of all highest weights of irreducible representations forms a cone

$$
X^{+}=\mathbb{Z} \times \mathbb{Z} \times \mathbb{N}^{n-2} \subseteq X
$$

(provided $n>1$ ). Here, the first factor of $\mathbb{Z}$ corresponds to the powers of the standard representation on $U(1)$ while the second factor of $\mathbb{Z}$ corresponds to the powers of the determinant representation on $U(n-1)$. The factor $\mathbb{N}^{n-2}$ is the space of weights of irreducible representations of $S U(n-1)$. 
Associated to $V_{\kappa}$ is a holomorphic vector bundle $\mathcal{V}_{\kappa}$ on $S h\left(K^{p}\right)_{\mathbb{C}}$, given by the Borel construction.

$$
\begin{gathered}
\mathcal{V}_{\kappa}=G U\left(\mathbb{Z}_{(p)}\right)^{+} \backslash\left(G U\left(\mathbb{A}^{p, \infty}\right) / K^{p} \times U(\mathbb{R}) \times_{K_{\infty}} V_{\kappa}\right) \\
\downarrow \\
S h\left(K^{p}\right)_{\mathbb{C}}=G U\left(\mathbb{Z}_{(p)}\right)^{+} \backslash\left(G U\left(\mathbb{A}^{p, \infty}\right) / K^{p} \times U(\mathbb{R}) / K_{\infty}\right)
\end{gathered}
$$

By a weakly holomorphic automorphic form of weight $\kappa$ we shall mean a holomorphic section of $\mathcal{V}_{\kappa}$. A holomorphic automorphic form of weight $\kappa$ is a weakly holomorphic automorphic form which satisfies certain growth conditions [Bor66]. If $S h\left(K^{p}\right)_{\mathbb{C}}$ is compact (i.e. if $B$ is a division algebra - see Theorem 6.6.2) then these growth conditions are always satisfied, and every weakly holomorphic automorphic form is holomorphic.

Let $\omega_{F}$ denote the line bundle $\left(\epsilon \operatorname{Lie} A^{+}\right)^{*}$ on $S h\left(K^{p}\right)_{F}$. Then $\omega_{F}$ restricts to $\omega$ on $\operatorname{Sh}\left(K^{p}\right)_{F_{u}}$. The pullback $\omega_{\mathbb{C}}$ of $\omega_{F}$ to $S h\left(K^{p}\right)_{\mathbb{C}}$ is the line bundle whose fiber over

$$
[g, J] \in G U\left(\mathbb{Z}_{(p)}\right)^{+} \backslash\left(G U\left(\mathbb{A}^{p, \infty}\right) / K^{p} \times \mathcal{H}\right) \cong S h\left(K^{p}\right)_{\mathbb{C}}
$$

is given by

$$
\left(\omega_{\mathbb{C}}\right)_{[g, J]}=\left(\operatorname{Lie} A_{J}^{+}\right)^{*}=\left(W_{J}^{+}\right)^{*} .
$$

We therefore have the following lemma.

LEMmA 9.4.1. There is an isomorphism of vector bundles $\omega_{\mathbb{C}}^{\otimes k} \cong \mathcal{V}_{-\underline{k}}$, where $-\underline{k}$ is the weight

$$
(-k, \underbrace{0, \ldots, 0}_{n-1}) \in X^{+} .
$$

We therefore have a zig-zag

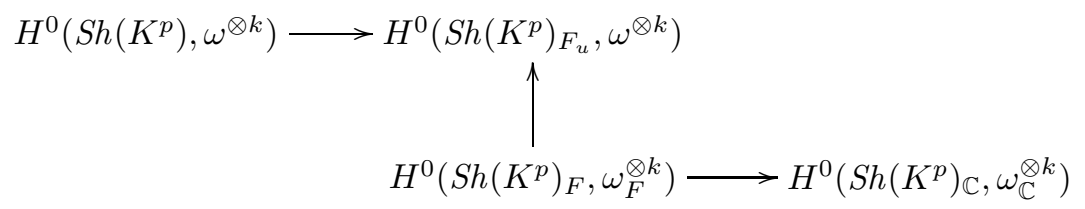

relating the $E_{2}$-term of the descent spectral sequence for $T A F_{K^{p}}$ (8.3.1) with the space of weakly holomorphic automorphic forms of weight $-\underline{k}$. 



\section{CHAPTER 10}

\section{Smooth $G$-spectra}

In this chapter we introduce the notion of a smooth $G$-spectrum for a totally disconnected locally compact group $G$. We shall see in the next chapter that the collection of spectra $\left\{T A F\left(K^{p}\right)\right\}$, as $K^{p}$ varies over the compact open subgroups of $G=G U\left(\mathbb{A}^{p, \infty}\right)$, gives rise to a smooth $G$-spectrum $V_{G U}$.

\subsection{Smooth $G$-sets}

Recall that a $G$-set $X$ is smooth if the stabilizer of every $x \in X$ is open. Such a $G$-set $X$ then satisfies

$$
X=\underset{H \leq \underline{o}_{0} G}{\lim _{H}} X^{H}
$$

where $H$ runs over the open subgroups of $G$. Let $\operatorname{Set}_{G}^{s m}$ be the category whose objects are the smooth $G$-sets and whose morphisms are $G$-equivariant maps.

Fix an infinite cardinality $\alpha$ larger than the cardinality of $G$. Let $\operatorname{Set}_{G}^{s m, \alpha}$ be the (essentially) small subcategory of $\operatorname{Set}_{G}^{s m}$ consisting of those smooth $G$-sets which have cardinality less than $\alpha$. We may endow $\operatorname{Set}_{G}^{s m, \alpha}$ with the structure of a Grothendieck topology by declaring that surjections are covers. The only nontrivial thing to verify is that $\operatorname{Set}_{G}^{s m, \alpha}$ contains pullbacks, but this is accomplished by the following lemma.

Lemma 10.1.1. Suppose that $H_{1}$ and $H_{2}$ are open subgroups of $H$, an open subgroup of $G$. Then the following diagram is a pullback diagram of smooth $G$-sets.

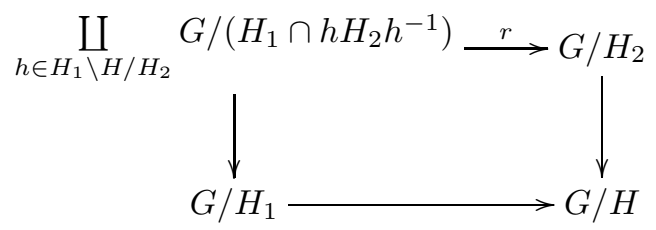

Here all of the maps are the evident surjections except for the map $r$, which is given by the following formula:

$$
r\left(g\left(H_{1} \cap h H_{2} h^{-1}\right)\right)=g h H_{2} .
$$

The site $\operatorname{Set}_{G}^{s m, \alpha}$ has enough points, with one unique point given by the filtering

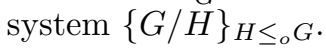


Every smooth $G$-set is a disjoint union of smooth $G$-orbits, and (since $G$-orbits are small) the natural map

$$
\prod_{i} \coprod_{j} \operatorname{Map}_{G}\left(G / H_{i}, G / H_{j}\right) \rightarrow \operatorname{Map}_{G}\left(\coprod_{i} G / H_{i}, \coprod_{j} G / H_{j}\right)
$$

is an isomorphism. Therefore, to describe the morphisms in $\operatorname{Set}_{G}^{s m}$ it suffices to describe the morphisms between smooth $G$-orbits. Let $H$ and $H^{\prime}$ be open subgroups of $G$, and consider the subset

$$
{ }_{H} N_{H^{\prime}}=\left\{g \in G: g^{-1} H g \leq H^{\prime}\right\} .
$$

We have an isomorphism

$$
\operatorname{Map}_{G}\left(G / H, G / H^{\prime}\right) \cong\left(H \backslash{ }_{H} N_{H^{\prime}} / H^{\prime}\right)^{o p}
$$

where for $g \in{ }_{H} N_{H^{\prime}}$, the double coset $H g H^{\prime}$ corresponds to the map

$$
R_{g}: x H \mapsto x g H^{\prime} .
$$

Suppose that $\mathcal{X}$ is a sheaf on the site $\operatorname{Set}_{G}^{s m, \alpha}$. Then the colimit

$$
X=\lim _{H \leq o} \mathcal{X}(G / H)
$$

taken over open subgroups $H$ is a smooth $G$-set with $H$-fixed points

$$
X^{H}=\mathcal{X}(G / H) \text {. }
$$

Conversely, any smooth $G$-set $X$ gives rise to a sheaf $\mathcal{X}$ on $\operatorname{Set}_{G}^{s m, \alpha}$ whose sections on a $G$-set $S=\amalg_{i} G / H_{i}$ are given by

$$
\mathcal{X}(S)=\prod_{i} X^{H_{i}}
$$

These constructions give the following lemma.

Lemma 10.1.4. The category of smooth $G$-sets is equivalent to the category of sheaves of sets on the site $\mathrm{Set}_{G}^{\text {sm, } \alpha}$.

There is an adjoint pair $\left(U,(-)^{s m}\right)$ of functors

$$
U: \operatorname{Set}_{G}^{s m} \leftrightarrows \operatorname{Set}_{G}:(-)^{s m}
$$

between the category of smooth $G$-sets and the category of all $G$-sets, where

$$
\begin{aligned}
& U(X)=X, \\
& X^{s m}=\underset{H \leq_{o} G}{\lim _{\longrightarrow}} X^{H} .
\end{aligned}
$$

The category of smooth $G$-sets is complete and cocomplete, with colimits formed in the underlying category of sets, and limits formed by applying the functor $(-)^{s m}$ to the underlying limit of sets. Finite limits are formed in the underlying category of sets.

REMARK 10.1.5. The properties listed above make the adjoint pair $\left(U,(-)^{s m}\right)$ a geometric morphism of topoi. 


\subsection{The category of simplicial smooth $G$-sets}

It is convenient to construct model categories of discrete $G$-spaces and discrete $G$-spectra for a profinite group $G$ by considering the category of simplicial (pre)sheaves of sets on the site of finite discrete $G$-spaces (see, for instance [Jar97]). An alternative, and equivalent approach, was proposed by Goerss [Goe95], who considered simplicial objects in the category of discrete $G$-sets. In this section we modify these frameworks to the case of $G$ a totally disconnected locally compact group. We investigate these model structures in some detail in preparation for some technical applications in Chapter 13.

Definition 10.2.1. A simplicial smooth $G$-set is a simplicial object in the category $\operatorname{Set}_{G}^{s m}$.

Consider the following categories:

$$
\begin{aligned}
s \operatorname{Set}_{G}^{s m} & =\operatorname{simplicial} \text { smooth } G \text {-sets, } \\
s \operatorname{Pre}\left(\operatorname{Set}_{G}^{s m, \alpha}\right) & =\operatorname{simplicial} \text { presheaves of sets on } \operatorname{Set}_{G}^{s m, \alpha}, \\
s \operatorname{Shv}\left(\operatorname{Set}_{G}^{s m, \alpha}\right) & =\operatorname{simplicial} \text { sheaves of sets on } \operatorname{Set}_{G}^{s m, \alpha} .
\end{aligned}
$$

Consider the diagram of functors

$$
s \operatorname{Pre}\left(\operatorname{Set}_{G}^{s m, \alpha}\right) \underset{U}{\stackrel{\mathcal{L}^{2}}{\rightleftarrows}} s \operatorname{Shv}\left(\operatorname{Set}_{G}^{s m, \alpha}\right) \underset{R}{\stackrel{L}{\rightleftarrows}} s \operatorname{Set}_{G}^{s m}
$$

where

$$
\begin{aligned}
\mathcal{L}^{2} & =\text { sheafification, } \\
U & =\text { forgetful functor, } \\
L(\mathcal{X}) & =\lim _{H \leq_{o} G} \mathcal{X}(G / H), \\
R(X)\left(\amalg_{i} G / K_{i}\right) & =\prod_{i} X^{K_{i}} .
\end{aligned}
$$

The pairs $\left(\mathcal{L}^{2}, U\right)$ and $(L, R)$ are adjoint pairs of functors. Lemma 10.1.4 has the following corollary.

COROLlary 10.2.3. The adjoint pair $(L, R)$ gives an adjoint equivalence of categories.

The categories of simplicial presheaves and sheaves of sets admit injective local model structures such that the adjoint pair $\left(\mathcal{L}^{2}, U\right)$ forms a Quillen equivalence [Jar87]. The category $s \operatorname{Set}_{G}^{s m}$ inherits a Quillen equivalent model structure under the adjoint equivalence $(L, R)$. We shall call this induced model structure on $s \operatorname{Set}_{G}^{s m}$ the injective local model structure.

LEMMA 10.2.4. A map in $s \operatorname{Set}_{G}^{s m}$ is a weak equivalence (cofibration) in the injective local model structure if and only if it induces a weak equivalence (monomorphism) on the underlying simplicial set. 
Proof. The local weak equivalences in $s \operatorname{Pre}\left(\operatorname{Set}_{G}^{s m, \alpha}\right)$ and $s \operatorname{Shv}\left(\operatorname{Set}_{G}^{s m, \alpha}\right)$ are the stalkwise weak equivalences, and the functor $L$ gives the stalk. A cofibration of simplicial presheaves is a sectionwise monomorphism. The result for cofibrations follows immediately from the definition of the functor $L$, and the fact that for a simplicial sheaf $\mathcal{X}$, and open subgroups $U \leq V \leq G$, the induced map

$$
\mathcal{X}(G / V) \rightarrow \mathcal{X}(G / U)
$$

is a monomorphism.

The category of simplicial sheaves on $\operatorname{Set}_{G}^{s m, \alpha}$ also admits a projective local model structure [Bla01]. In this model structure, the weak equivalences are still the local (stalkwise) weak equivalences, but the projective cofibrations are generated by the inclusions of the representable simplicial sheaves: the generating cofibrations are morphisms of the form

$$
i_{n, Z}: \partial \Delta^{n} \times \operatorname{Map}_{G}(-, Z) \hookrightarrow \Delta^{n} \times \operatorname{Map}_{G}(-, Z)
$$

for $Z \in \operatorname{Set}_{G}^{s m, \alpha}$. The projective local fibrations are determined. The category of simplicial smooth $G$-sets inherits a projective local model structure from the adjoint equivalence $(L, R)$.

LEMma 10.2.5. A map in $s \operatorname{Set}_{G}^{s m}$ is a projective cofibration if and only if it is a monomorphism.

Proof. The class of projective cofibrations is generated by the monomorphisms

$$
L i_{n, Z}: Z \times \partial \Delta^{n} \hookrightarrow Z \times \Delta^{n}
$$

for $Z \in \operatorname{Set}_{G}^{s m, \alpha}$. It clearly suffices to consider the generating morphisms $L i_{n, G / H}$ for open subgroups $H \leq G$. Because the maps $L i_{n, G / H}$ are monomorphisms, every projective cofibration is a monomorphism. Conversely, given an inclusion $j: X \hookrightarrow$ $Y$ of simplicial smooth $G$-sets, we have

$$
Y=\underset{k}{\lim } Y^{[k]}
$$

where $Y^{[-1]}=X$ and $Y^{[k]}$ is given by the pushout

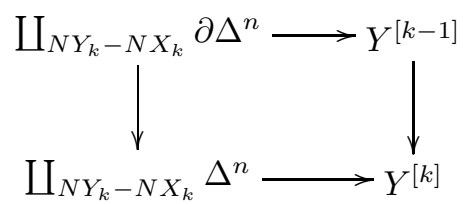

where $N X_{k}$ and $N Y_{k}$ are the (smooth $G$-sets of) nondegenerate $k$-simplices of $X$ and $Y$, respectively. This pushout may be rewritten in terms of $G$-orbits of nondegenerate $k$-simplices as follows.

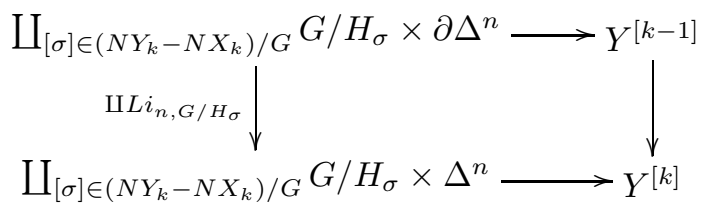


Here, for a representative $\sigma$ of an $G$-orbit $[\sigma]$, the group $H_{\sigma}$ is the (open) stabilizer of $\sigma$. We have shown that the class of monomorphisms is contained in the category of cofibrations generated by the morphisms $L i_{n, Z}$.

We have shown that the cofibrations and weak equivalences are the same in the injective and projective local model category structures on $s \operatorname{Set}_{G}^{s m}$, giving the following surprising corollary.

COROLLARY 10.2.6. The injective and projective local model category structures on $s \operatorname{Set}_{G}^{s m}$ agree.

It is shown in [Bla01] that a map between simplicial sheaves is a projective local fibration if and only if it is a projective local fibration on the level of underlying presheaves. In [DHI04, Thm 1.3], an explicit characterization of the projective fibrant presheaves is given. This fibrancy condition, when translated into $s \operatorname{Set}_{G}^{s m}$ using the functor $L$, gives the following characterization of the fibrant objects of $s \operatorname{Set}_{G}^{s m}$.

Lemma 10.2.7. A simplicial smooth $G$-set $X$ is fibrant if and only if:

(1) the $H$-fixed points $X^{H}$ is a Kan complex for every open subgroup $H \leq G$,

(2) for every open subgroup $H \leq G$ and every hypercover

$$
\cdots \coprod_{\alpha \in I_{2}} \widetilde{G / U_{\alpha, 2}} \Rightarrow \coprod_{\alpha \in I_{1}} \widetilde{G / U}{ }_{\alpha, 1} \Rightarrow \coprod_{\alpha \in I_{0}} \widetilde{G / U}{ }_{\alpha, 0} \rightarrow \widetilde{G / H}
$$

(where $\widetilde{G / U}$ denotes the representable sheaf associated to a smooth orbit $G / U)$ the induced map

$$
X^{H} \rightarrow \underset{\Delta}{\operatorname{holim}} \prod_{\alpha \in I_{\bullet}} X^{U_{\alpha, \bullet}}
$$

is a weak equivalence.

REMARK 10.2.8. Since filtered colimits of Kan complexes are Kan complexes, the underlying simplicial set of a fibrant simplicial smooth $G$-set is a Kan complex.

\subsection{The category of smooth $G$-spectra}

The methods of Section 10.2 extend in a straightforward manner to give model categories of smooth $G$-spectra, as investigated in the case of $G$ profinite by [Jar97] and [Dav06]. These smooth $G$-spectra are intended to generalize "naive equivariant stable homotopy theory": the fibrant objects will not distinguish between fixed points and homotopy fixed points.

Definition 10.3.1. A smooth $G$-spectrum is a Bousfield-Friedlander spectrum of smooth $G$-sets. It consists of a sequence $\left\{X_{i}\right\}_{i \geq 0}$ of pointed simplicial smooth $G$-sets together with $G$-equivariant structure maps

$$
\Sigma X_{i} \rightarrow X_{i+1} \text {. }
$$

REMARK 10.3.2. If $G$ is a profinite group, then the notion of a smooth $G$-spectrum coincides with that of a discrete $G$-spectrum studied in [Dav06]. 
Consider the following categories (where spectrum means Bousfield-Friedlander spectrum of simplicial sets):

$$
\begin{aligned}
\operatorname{Sp}_{G}^{s m} & =\text { category of smooth } G \text {-spectra, } \\
\operatorname{PreSp}\left(\operatorname{Set}_{G}^{s m, \alpha}\right) & =\text { category of presheaves of spectra on } \operatorname{Set}_{G}^{s m, \alpha}, \\
\operatorname{Shv} \operatorname{Sp}\left(\operatorname{Set}_{G}^{s m, \alpha}\right) & =\text { category of sheaves of spectra on } \operatorname{Set}_{G}^{s m, \alpha} .
\end{aligned}
$$

Just as in Section 10.2, we have a diagram of functors

$$
\operatorname{Pre} \operatorname{Sp}\left(\operatorname{Set}_{G}^{s m, \alpha}\right) \underset{U}{\stackrel{\mathcal{L}^{2}}{\rightleftarrows}} \operatorname{Shv} \operatorname{Sp}\left(\operatorname{Set}_{G}^{s m, \alpha}\right) \underset{R}{\stackrel{L}{\rightleftarrows}} \operatorname{Sp}_{G}^{s m}
$$

where

$$
\begin{aligned}
\mathcal{L}^{2} & =\text { sheafification, } \\
U & =\text { forgetful functor, } \\
L(\mathcal{X}) & =\lim _{\overrightarrow{H \leq_{o} G}} \mathcal{X}(G / H), \\
R(X)\left(\amalg_{i} G / K_{i}\right) & =\prod_{i} X^{K_{i}} .
\end{aligned}
$$

The pairs $\left(\mathcal{L}^{2}, U\right)$ and $(L, R)$ are adjoint pairs of functors, and the adjoint pair $(L, R)$ gives an adjoint equivalence of categories.

The categories of presheaves and sheaves of spectra admit injective local model structures such that the adjoint pair $\left(\mathcal{L}^{2}, U\right)$ forms a Quillen equivalence [GJ98]. The category $\mathrm{Sp}_{G}^{s m}$ inherits a Quillen equivalent model structure under the adjoint equivalence $(L, R)$.

Lemma 10.3.4. A map in $\mathrm{Sp}_{G}^{s m}$ is a weak equivalence (cofibration) if and only if it induces a stable equivalence (cofibration) on the underlying spectrum.

Proof. The weak equivalences in $\operatorname{PreSp}\left(\operatorname{Set}_{G}^{s m, \alpha}\right)$ and $\operatorname{Shv} \operatorname{Sp}\left(\operatorname{Set}_{G}^{s m, \alpha}\right)$ are the stalkwise stable equivalences. For a sheaf of spectra $\mathcal{E}$, the underlying spectrum of $L \mathcal{E}$ is the stalk of $\mathcal{E}$ at the (unique) point of the site $\operatorname{Set}_{G}^{s m, \alpha}$. An argument analogous to that given in [Dav06, Thm 3.6] proves the statement concerning cofibrations.

\subsection{Smooth homotopy fixed points}

For a smooth $G$-spectrum $X$, we define the fixed point spectrum by taking the fixed points level-wise

$$
\left(X^{G}\right)_{i}=\left(X_{i}\right)^{G} .
$$

The $G$-fixed point functor is right adjoint to the functor triv which associates to a spectrum the associated smooth $G$-spectrum with trivial $G$-action.

$$
\text { triv: } \mathrm{Sp} \leftrightarrows \mathrm{Sp}_{G}:(-)^{G}
$$

Since the functor triv preserves cofibrations and weak equivalences, we have the following lemma. 
LEMma 10.4.1. The adjoint functors $\left(\right.$ triv,$\left.(-)^{G}\right)$ form a Quillen pair.

Let $\beta_{G, X}: X \rightarrow X_{f G}$ denote a functorial fibrant replacement functor for the model category $\mathrm{Sp}_{G}^{s m}$, where $\beta_{G, X}$ is a trivial cofibration of smooth $G$-spectra. The homotopy fixed point functor $(-)^{h G}$ is the Quillen right derived functor of $(-)^{G}$, and is thus given by

$$
X^{h G}=\left(X_{f G}\right)^{G} .
$$

\subsection{Restriction, induction, and coinduction}

Fix an open subgroup $H$ of $G$. For a smooth $H$-set $Z$, we define the coinduced smooth $G$-set by

$$
\operatorname{CoInd}_{H}^{G} Z=\operatorname{Map}_{H}(G, Z)^{s m}:=\underset{U \leq_{o} G}{\lim _{\longrightarrow}} \operatorname{Map}_{H}(G / U, Z)
$$

where the colimit is taken over open subgroups. An element $g \in G$ sends an element $\alpha \in \operatorname{Map}_{H}(G / U, Z)$ to an element $g \cdot \alpha \in \operatorname{Map}_{H}\left(G / g U g^{-1}, Z\right)$ by the formula

$$
(g \cdot \alpha)\left(g^{\prime}\left(g U g^{-1}\right)\right)=\alpha\left(g^{\prime} g U\right) .
$$

This construction extends to simplicial smooth $G$-sets and smooth $G$-spectra in the obvious manner to give a functor

$$
\operatorname{CoInd}_{H}^{G}: \mathrm{Sp}_{H}^{s m} \rightarrow \mathrm{Sp}_{G}^{s m} .
$$

Let $\operatorname{Res}_{H}^{G}$ be the restriction functor:

$$
\operatorname{Res}_{H}^{G}: \operatorname{Sp}_{G}^{s m} \rightarrow \operatorname{Sp}_{H}^{s m} .
$$

The functors $\left(\operatorname{Res}_{H}^{G}, \operatorname{CoInd}_{H}^{G}\right)$ form an adjoint pair. Since $\operatorname{Res}_{H}^{G}$ preserves cofibrations and weak equivalences, we have the following lemma.

LEMmA 10.5.1. The adjoint functors $\left(\operatorname{Res}_{H}^{G}, \operatorname{CoInd}_{H}^{G}\right)$ form a Quillen pair.

The Quillen pair $\left(\operatorname{Res}_{H}^{G}\right.$, $\left.\operatorname{CoInd}_{H}^{G}\right)$ gives rise to a derived pair $\left(L \operatorname{Res}_{H}^{G}, R \operatorname{CoInd}_{H}^{G}\right)$. Since the functor $\operatorname{Res}_{H}^{G}$ preserves all weak equivalences, there are equivalences $L \operatorname{Res}_{H}^{G} X \simeq \operatorname{Res}_{H}^{G} X$ for all smooth $G$-spectra $X$.

Lemma 10.5.2 (Shapiro's Lemma). Let $X$ be a smooth $H$-spectrum, and suppose that $H$ is an open subgroup of $G$. Then there is an equivalence

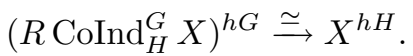

Proof. The lemma follows immediately from the following commutative diagram of functors.

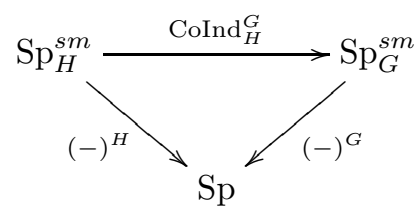


Define the induction functor on a smooth $H$-spectrum $Y$ to be the Borel construction

$$
\operatorname{Ind}_{H}^{G} Y=G_{+} \wedge_{H} Y .
$$

Here, the Borel construction is taken regarding $G$ and $H$ as being discrete groups, but this is easily seen to produce a smooth $G$-spectrum since $H$ is an open subgroup of $G$. The induction and restriction functors form an adjoint pair $\left(\operatorname{Ind}_{H}^{G}, \operatorname{Res}_{H}^{G}\right)$

$$
\operatorname{Ind}_{H}^{G}: \operatorname{Sp}_{H}^{s m} \leftrightarrows \operatorname{Sp}_{G}^{s m}: \operatorname{Res}_{H}^{G}
$$

Since non-equivariantly we have an isomorphism

$$
\operatorname{Ind}_{H}^{G} Y \cong G / H_{+} \wedge Y
$$

we have the following lemma.

LEMmA 10.5.3. The functor $\operatorname{Ind}_{H}^{G}$ preserves cofibrations and weak equivalences, and therefore $\operatorname{Res}_{H}^{G}$ preserves fibrant objects.

Definition 10.5.4. Let $X$ be a smooth $G$-spectrum. Define the smooth homotopy $H$-fixed points of $X$ by

$$
X^{h H}:=\left(\operatorname{Res}_{H}^{G} X\right)^{h H}
$$

Lemma 10.5.3 has the following corollary.

Corollary 10.5.5. For a smooth $G$-spectrum $X$, the spectrum $X^{h H}$ is equivalent to the $H$-fixed points $\left(X_{f G}\right)^{H}$.

\subsection{Descent from compact open subgroups}

In this section we will explain how the homotopy type of a smooth $G$-spectrum can be reconstructed using only its homotopy fixed points for compact open subgroups of $G$ (Construction 10.6.3). This construction will be used in Section 11.1 to construct a smooth $G U\left(\mathbb{A}^{p, \infty}\right)$-spectrum $V_{G U}$ from the collection of spectra $\left\{T A F\left(K^{p}\right)\right\}$, where $K^{p}$ ranges over the compact open subgroups of $G U\left(\mathbb{A}^{p, \infty}\right)$.

Let Set $_{G}^{c o, \alpha}$ be the full subcategory of $\operatorname{Set}_{G}^{s m, \alpha}$ consisting of objects $X$ whose stabilizers are all compact. Every object $Z$ of $\operatorname{Set}_{G}^{c o, \alpha}$ is therefore isomorphic to a disjoint union of $G$-orbits

$$
Z=\coprod_{i} G / K_{i}
$$

where the subgroups $K_{i}$ are compact and open. Lemma 10.1.1 implies that $\operatorname{Set}^{c o, \alpha}$ is a Grothendieck site, with covers given by surjections. The site $\operatorname{Set}_{G}^{c o, \alpha}$ also possesses enough points, with a unique point given by the filtering system $\{G / K\}_{K}$ where $K$ ranges over the compact open subgroups of $G$.

Just as in Section 10.3, there is an adjoint equivalence $\left(L^{\prime}, R^{\prime}\right)$

$$
L^{\prime}: \operatorname{Shv} \operatorname{Sp}\left(\operatorname{Set}_{G}^{c o, \alpha}\right) \leftrightarrows \operatorname{Sp}_{G}^{s m}: R^{\prime}
$$


where

$$
\begin{aligned}
L^{\prime} \mathcal{X} & =\lim _{K \leq_{c o} G} \mathcal{X}(G / K), \\
\left(R^{\prime} X\right)\left(\amalg_{i} G / K_{i}\right) & =\prod_{i} X^{K_{i}} .
\end{aligned}
$$

The colimit in the definition of $L^{\prime}$ is taken over compact open subgroups of $G$.

The adjoint equivalence $\left(L^{\prime}, R^{\prime}\right)$ induces a model structure on $\mathrm{Sp}_{G}^{s m}$ from the Jardine model structure on $\operatorname{Shv} \operatorname{Sp}\left(\operatorname{Set}_{G}^{c o, \alpha}\right)$. Precisely the same arguments proving Lemma 10.3.4 apply to prove the following lemma.

LEMma 10.6.1. In the model structure on $\mathrm{Sp}_{G}^{s m}$ induced from the Jardine model structure on $\mathrm{Shv} \operatorname{Sp}\left(\mathrm{Set}_{G}^{c o, \alpha}\right.$ ), a map is a weak equivalence (cofibration) if and only if it is a weak equivalence (cofibration) on the underlying spectrum.

COROLlary 10.6.2. The model structure on $\mathrm{Sp}_{G}^{s m}$ induced from the Jardine model structure on $\mathrm{Shv} \operatorname{Sp}\left(\operatorname{Set}_{G}^{c o, \alpha}\right)$ is identical to that induced from the the model structure on $\operatorname{Shv} \operatorname{Sp}\left(\operatorname{Set}_{G}^{s m, \alpha}\right)$.

Construction 10.6.3. Begin with a presheaf of spectra $\mathcal{X}$ on the site $\operatorname{Set}_{G}^{c o, \alpha}$. We associate to $\mathcal{X}$ a smooth $G$-spectrum

$$
V_{\mathcal{X}}=L^{\prime}\left(\mathcal{L}^{2} \mathcal{X}\right)_{f}
$$

the functor $L^{\prime}$ applied to the fibrant replacement of the sheafification of $\mathcal{X}$.

Lemma 10.6.4. The smooth $G$-spectrum $V_{\mathcal{X}}$ is fibrant.

Proof. The fibrant objects in $\mathrm{Sp}_{G}^{s m}$ are precisely those objects $X$ for which $R^{\prime} X$ is fibrant in $\operatorname{Shv} \operatorname{Sp}\left(\operatorname{Set}_{G}^{c o, \alpha}\right)$. Since $\left(L^{\prime}, R^{\prime}\right)$ form an equivalence of categories, there is an isomorphism

$$
R^{\prime} V_{\mathcal{X}}=R^{\prime} L^{\prime}\left(\mathcal{L}^{2} \mathcal{X}\right)_{f} \cong\left(\mathcal{L}^{2} \mathcal{X}\right)_{f}
$$

Lemma 10.6.5. Suppose that $\mathcal{X}$ is a fibrant presheaf of spectra on $\operatorname{Set}_{G}^{c o, \alpha}$, and that $K$ is a compact open subgroup of $G$. Then there is an equivalence of spectra

$$
\mathcal{X}(G / K) \stackrel{\simeq}{\longrightarrow} V_{\mathcal{X}}^{K} \text {. }
$$

Proof. Consider the Quillen equivalence given by the adjoint pair

$$
\mathcal{L}^{2}: \operatorname{Pre} \operatorname{Sp}\left(\operatorname{Set}_{G}^{c o, \alpha}\right) \leftrightarrows \operatorname{Shv} \operatorname{Sp}\left(\operatorname{Set}_{G}^{c o, \alpha}\right): U
$$

where $\mathcal{L}^{2}$ is the sheafification functor and $U$ is the forgetful functor. The functor $U$ preserves all weak equivalences.

Define $\phi$ to be the composite

$$
\phi: \mathcal{X} \stackrel{\eta}{\rightarrow} U \mathcal{L}^{2} \mathcal{X} \stackrel{U(\alpha)}{\longrightarrow} U\left(\mathcal{L}^{2} \mathcal{X}\right)_{f}
$$

in the category $\operatorname{Pre} \operatorname{Sp}\left(\operatorname{Set}_{G}^{c o, \alpha}\right)$, where $\eta$ is the unit of the adjunction and $\alpha$ is the fibrant replacement morphism for the sheaf $\mathcal{L}^{2} \mathcal{X}$. The map $\eta$ is always a weak equivalence, and, since $U$ preserves all weak equivalences, the map $U(\alpha)$ is a weak equivalence. Thus $\phi$ is a weak equivalence of presheaves of spectra. 
The functor $U$, being the right adjoint of a Quillen pair, preserves fibrant objects, and thus $\phi$ is a weak equivalence between fibrant objects. The sections functor

$$
\begin{aligned}
\Gamma_{G / K}: \operatorname{PreSp}\left(\operatorname{Set}_{G}^{c o, \alpha}\right) & \rightarrow \operatorname{Sp} \\
\mathcal{E} & \mapsto \mathcal{E}(G / K)
\end{aligned}
$$

is also the right adjoint of a Quillen pair, and hence preserves weak equivalences between fibrant objects. We therefore have a string of equivalences

$$
\begin{aligned}
\mathcal{X}(G / K) \stackrel{\Gamma_{G / K} \phi}{\simeq} & U\left(\mathcal{L}^{2} \mathcal{X}\right)_{f}(G / K) \\
& =\left(\mathcal{L}^{2} \mathcal{X}\right)_{f}(G / K) \\
& \left.\cong\left(R^{\prime} L^{\prime} \mathcal{L}^{2} \mathcal{X}\right)_{f}\right)(G / K) \\
& =V_{\mathcal{X}}^{K} .
\end{aligned}
$$

\subsection{Transfer maps and the Burnside category}

In this section we will construct transfer maps between the homotopy fixed points of compact open subgroups. We will then explain how this extra structure assembles to give this collection of homotopy fixed point spectra the structure of a Mackey functor.

Construction of transfers. We will now explain how to use Shapiro's Lemma to construct transfer maps for smooth $G$-spectra.

Suppose $K$ is a compact open subgroup of $G$ with open subgroup $K^{\prime}$. There is a continuous based map $K_{+} \rightarrow K_{+}^{\prime}$, equivariant with respect to the left and right $K^{\prime}$-actions, given by

$$
k \mapsto \begin{cases}k & \text { if } k \in K^{\prime} \\ * & \text { otherwise }\end{cases}
$$

Given a smooth $K^{\prime}$-spectrum $X$, this gives rise to a map of $K^{\prime}$-spectra

$$
X \cong \operatorname{Map}_{K^{\prime}, *}\left(K_{+}^{\prime}, X\right)^{s m} \rightarrow \operatorname{Map}_{K^{\prime}, *}\left(K_{+}, X\right)^{s m} \cong \operatorname{Res}_{K^{\prime}}^{K} \operatorname{CoInd}_{K^{\prime}}^{K} X .
$$

The adjoint of this map is a natural map of $K$-spectra

$$
i_{K^{\prime}}^{K}: \operatorname{Ind}_{K^{\prime}}^{K} X \rightarrow \operatorname{CoInd}_{K^{\prime}}^{K} X .
$$

On the level of underlying spectra, this map is an inclusion

$$
\bigvee_{K / K^{\prime}} X \rightarrow \prod_{K / K^{\prime}} X
$$

and hence is a natural weak equivalence of smooth $K$-spectra.

Consider the counit map

$$
\varepsilon_{K^{\prime}}^{K}: \operatorname{Ind}_{K^{\prime}}^{K} \operatorname{Res}_{K^{\prime}}^{K} X \rightarrow X
$$


Application of the right derived functor of smooth coinduction gives a diagram of maps

$$
R \operatorname{CoInd}_{K^{\prime}}^{G} X \leftarrow R \operatorname{CoInd}_{K}^{G} \operatorname{Ind}_{K^{\prime}}^{K} X \rightarrow R \operatorname{CoInd}_{K}^{G} X
$$

of smooth $G$-spectra. Upon taking homotopy fixed points, Lemma 10.5.2 gives rise to natural maps

$$
X^{h K^{\prime}} \stackrel{\sim}{\leftarrow}\left(\operatorname{Ind}_{K^{\prime}}^{K} \operatorname{Res}_{K^{\prime}}^{G} X\right)^{h K} \rightarrow X^{h K} .
$$

Definition 10.7.1. The transfer associated to the inclusion of $K^{\prime}$ in $K$ is the corresponding map $\operatorname{Tr}_{K^{\prime}}^{K}: X^{h K^{\prime}} \rightarrow X^{h K}$ in the homotopy category.

The Burnside category. The treatment in this section follows [Min99]. Define the Burnside category $\mathcal{M}_{G}^{c o}$ of $G$-sets to be the a category with objects given by

$$
\operatorname{Ob}\left(\mathcal{M}_{G}^{c o}\right)=\left\{Z \in \operatorname{Set}_{G}^{c o, \alpha}:|Z / G|<\infty\right\} .
$$

Thus the objects of $\mathcal{M}_{G}^{c o}$ are $G$-sets with finitely many orbits and compact open stabilizers.

We now define the morphisms. Let $S, T$ be objects of $\mathcal{M}_{G}^{c o}$. Let $\operatorname{Cor}(S, T)$ denote the set of correspondences given by isomorphism classes of diagrams in $\operatorname{Set}_{G}^{c o}$ of the form

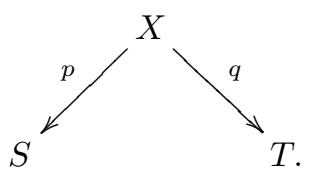

The set $\operatorname{Cor}(S, T)$ admits the structure of a commutative monoid through disjoint unions:

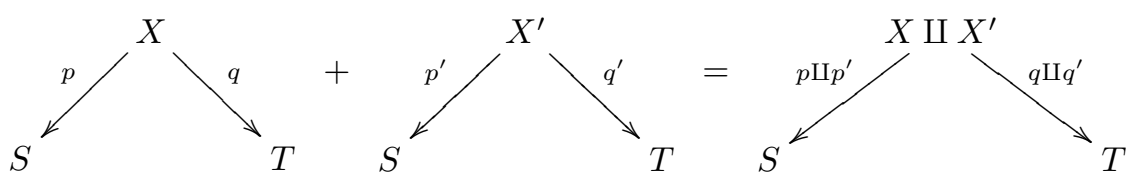

The morphisms of the category $\mathcal{M}_{G}^{c o}$ from $S$ to $T$ are defined to be the Grothendieck group of the monoid $\operatorname{Cor}(S, T)$ :

$$
\operatorname{Hom}_{\mathcal{M}_{G}^{c o}}(S, T)=\operatorname{Groth}(\operatorname{Cor}(S, T)) .
$$

Composition in $\mathcal{M}_{G}^{c o}$ is given by:

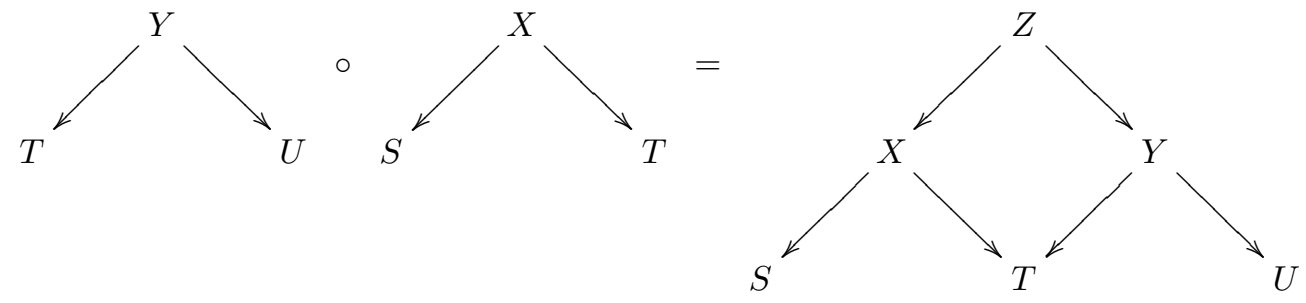

where $Z$ is the pullback $X \times_{T} Y$. 
The morphisms of $\mathcal{M}_{G}^{c o}$ are generated by the right multiplication by $g$ maps

$$
\rho_{g}: G / g K g^{-1} \rightarrow G / K,
$$

the projections

$$
r_{K^{\prime}}^{K}: G / K^{\prime} \rightarrow G / K,
$$

and the transfers

$$
i_{K^{\prime}}^{K}: G / K \rightarrow G / K^{\prime}
$$

for $g \in G, K^{\prime}<K$, satisfying the following relations.

(1) $\rho_{g} \circ \rho_{h}=\rho_{h g}$

(2) $r_{K^{\prime}}^{K} \circ r_{K^{\prime \prime}}^{K^{\prime}}=r_{K^{\prime \prime}}^{K}$

(3) $i_{K^{\prime \prime}}^{K^{\prime}} \circ i_{K^{\prime}}^{K}=i_{K^{\prime \prime}}^{K}$

(4) $\rho_{g} \circ r_{g K^{\prime} g^{-1}}^{g K g^{-1}}=r_{K^{\prime}}^{K} \circ \rho_{g}$

(5) $\rho_{g} \circ i_{g K^{\prime} g^{-1}}^{g K g^{-1}}=i_{K^{\prime}}^{K} \circ \rho_{g}$

(6) $i_{L}^{H} \circ r_{K}^{H}=\sum_{K x L} r_{x^{-1} K x \cap L}^{L} \rho_{x} \circ i_{K \cap x L x^{-1}}^{K}$

Definition 10.7.2. A Mackey functor (for the group $G$ ) is an additive functor

$$
\mathcal{M}_{G}^{c o} \rightarrow \text { Abelian groups }
$$

which takes coproducts to direct sums.

The Mackey functor associated to a smooth $G$-spectrum. For a smooth $G$-spectrum $X$ we get an induced functor

$$
\mathcal{X}:\left(\mathcal{M}_{G}^{c o}\right)^{o p} \rightarrow \mathrm{Ho}(\mathrm{Sp})
$$

from the Burnside category to the homotopy category of spectra as follows. On objects, we define

$$
\mathcal{X}\left(\amalg_{i} G / K_{i}\right)=\prod_{i} X^{h K_{i}} .
$$

To a correspondence of the form

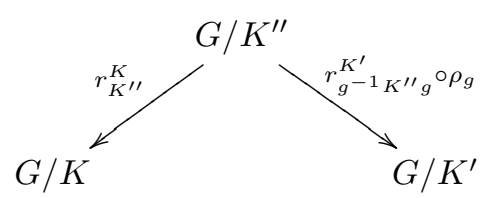

we assign the composite

$$
X^{h K^{\prime}} \stackrel{\operatorname{Res}_{g^{-1} K^{\prime \prime} g}^{K^{\prime}}}{\longrightarrow} X^{h g^{-1} K^{\prime \prime} g} \stackrel{g_{*}}{\longrightarrow} X^{h K^{\prime \prime}} \stackrel{\operatorname{Tr}_{K^{\prime \prime}}^{K}}{\longrightarrow} X^{h K} .
$$

Every correspondence between $G / K$ and $G / K^{\prime}$ is a disjoint union of correspondences of the form (10.7.3), and we assign to it the corresponding sum of morphisms in the homotopy category of spectra. Thus we have defined a map of commutative monoids

$$
\operatorname{Cor}\left(G / K, G / K^{\prime}\right) \rightarrow \operatorname{Hom}_{\mathrm{Ho}(\mathrm{Sp})}\left(X^{h K^{\prime}}, X^{h K}\right)
$$

which extends to a homomorphism

$$
\operatorname{Hom}_{\mathcal{M}_{G}^{c o}}\left(G / K, G / K^{\prime}\right) \rightarrow \operatorname{Hom}_{\mathrm{Ho}(\mathrm{Sp})}\left(X^{h K^{\prime}}, X^{h K}\right) .
$$


Extending additively we have homomorphisms

$$
\operatorname{Hom}_{\mathcal{M}_{G}^{c o}}(S, T) \rightarrow \operatorname{Hom}_{\mathrm{Ho}(\mathrm{Sp})}(\mathcal{X}(T), \mathcal{X}(S))
$$

for every pair of objects $S, T \in \mathcal{M}_{G}^{c o}$.

In view of the definition of composition in $\mathcal{M}_{G}^{c o}$, the following lemma implies the functoriality of $\mathcal{X}$. The lemma is easily verified using the definition of transfer.

Lemma 10.7.4. Suppose that $H_{1}$ and $H_{2}$ are open subgroups of $H$, a compact open subgroup of $G$. Consider the following pullback diagram of Lemma 10.1.1.

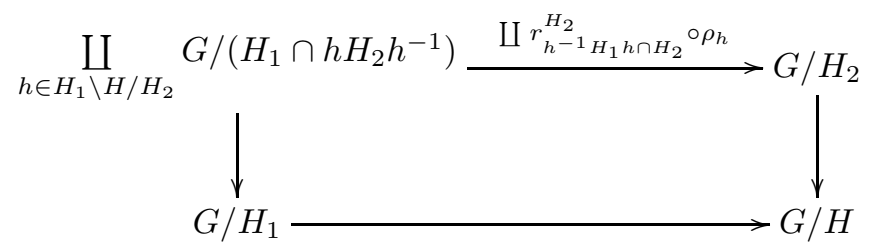

Then the following diagram commutes in $\mathrm{Ho}(\mathrm{Sp})$.

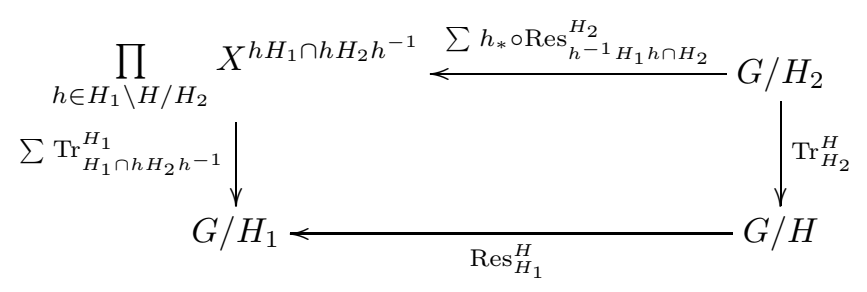

The previous relations imply the following.

Proposition 10.7.5. The conjugation, restriction, and transfer maps satisfy the following relations.

(1) $h_{*} \circ g_{*}=(h g)_{*}$

(2) $\operatorname{Res}_{K^{\prime \prime}}^{K^{\prime}} \circ \operatorname{Res}_{K^{\prime}}^{K}=\operatorname{Res}_{K^{\prime \prime}}^{K}$

(3) $\operatorname{Tr}_{K^{\prime}}^{K} \circ \operatorname{Tr}_{K^{\prime \prime}}^{K^{\prime}}=\operatorname{Tr}_{K^{\prime \prime}}^{K}$

(4) $\operatorname{Res}_{g K^{\prime} g^{-1}}^{g K g^{-1}} \circ g_{*}=g_{*} \circ \operatorname{Res}_{K^{\prime}}^{K}$

(5) $\operatorname{Tr}_{g K^{\prime} g^{-1}}^{g K g^{-1}} \circ g_{*}=g_{*} \circ \operatorname{Tr}_{K^{\prime}}^{K}$

(6) $\operatorname{Res}_{K}^{H} \circ \operatorname{Tr}_{L}^{H}=\sum_{K x L} \operatorname{Tr}_{K \cap x L x^{-1}}^{K} \circ x_{*} \circ \operatorname{Res}_{x^{-1} K x \cap L}^{L}$

Corollary 10.7.6. The functor

$$
\begin{aligned}
\pi_{*} \mathcal{X}(-): \mathcal{M}_{G}^{c o} & \rightarrow \text { Abelian groups } \\
\amalg_{i} G / K_{i} & \mapsto \bigoplus_{i} \pi_{*}\left(X^{h K_{i}}\right)
\end{aligned}
$$

is a Mackey functor.

For any $G$-module $M$, the fixed points form a Mackey functor.

$$
\begin{aligned}
M^{(-)}:\left(\mathcal{M}_{G}^{c o}\right)^{o p} & \rightarrow \text { Abelian Groups } \\
\amalg_{i} G / K_{i} & \mapsto \bigoplus_{i} M^{K_{i}}
\end{aligned}
$$


The transfers

$$
\operatorname{Tr}_{K^{\prime}}^{K}: M^{K^{\prime}} \rightarrow M^{K}
$$

are given by

$$
\operatorname{Tr}_{K^{\prime}}^{K}(m)=\sum_{[g] \in K / K^{\prime}} g m
$$

Consider the canonical "edge homomorphisms"

$$
\epsilon_{K}: \pi_{*}\left(X^{h K}\right) \rightarrow \pi_{*}(X)^{K} .
$$

The following lemma is easily verified by comparing the definitions of transfers.

LEMMA 10.7.7. The homomorphisms $\epsilon_{(-)}$assemble to give a natural transformation of Mackey functors

$$
\epsilon_{(-)}: \pi_{*}\left(X^{h(-)}\right) \rightarrow \pi_{*}(X)^{(-)}
$$




\section{CHAPTER 11}

\section{Operations on TAF}

In this chapter we will describe three classes of cohomology operations, which extend classical operations in the theory of automorphic forms.

(1) Restriction: For compact open subgroups $K^{\prime p} \leq K^{p}$, a map of $E_{\infty^{-}}$ ring spectra

$$
\operatorname{Res}_{K^{\prime p}}^{K^{p}}: \operatorname{TAF}\left(K^{p}\right) \rightarrow T A F\left(K^{p}\right) .
$$

(2) Action of $G U\left(\mathbb{A}^{p, \infty}\right)$ : For $g \in G U\left(\mathbb{A}^{p, \infty}\right)$ and $K^{p}$ as above, a map of $E_{\infty}$-ring spectra

$$
g_{*}: T A F\left(K^{p}\right) \rightarrow T A F\left(g K^{p} g^{-1}\right) .
$$

(3) Transfer: For $K^{p}$ and $K^{\prime p}$ as above, a map in the homotopy category spectra

$$
\operatorname{Tr}_{K^{\prime}}^{K^{p}}: T A F\left(K^{\prime p}\right) \rightarrow T A F\left(K^{p}\right) .
$$

Operations of type (1) and (2) may be most elegantly formulated as a functor on an orbit category, as we describe in Section 11.1. This structure gives rise to a smooth $G U\left(\mathbb{A}^{p, \infty}\right)$-spectrum $V_{G U}$. The operations of type (3) then come automatically (see Section 10.7). All three types of operations encode the fact that $T A F(-)$ induces a Mackey functor from the Burnside category into the homotopy category of spectra.

The relationship of this Mackey functor structure to the theory of Hecke algebras is discussed in Section 11.2. While we are able to lift Hecke operators on automorphic forms to cohomology operations on $\operatorname{TAF}\left(K^{p}\right)$, we are unable to determine in general if this induces an action of the corresponding Hecke algebra (we do treat a very restrictive case in Propositions 11.2.3 and 11.2.6). We are not advocating that having the Hecke algebra act on $T A F(K)$ is necessarily the "right" point of view. The most important structure is the Mackey functor structure.

\subsection{The $E_{\infty}$-action of $G U\left(\mathbb{A}^{p, \infty}\right)$}

Let $\operatorname{Set}_{G U\left(\mathbb{A}^{p}, \infty\right)}^{c o, \alpha}$ be the Grothendieck site of Section 10.6.

Proposition 11.1.1. The assignment

$$
\coprod_{i} G U\left(\mathbb{A}^{p, \infty}\right) / K_{i}^{p} \mapsto \prod_{i} T A F\left(K_{i}^{p}\right)
$$

gives a presheaf

$$
T A F(-):\left(\operatorname{Set}_{G U\left(\mathbb{A}^{p, \infty}\right)}^{c o, \alpha}\right)^{o p} \rightarrow E_{\infty} \text {-ring spectra. }
$$


ProOF. In light of the isomorphism (10.1.2), it suffices to establish functoriality on orbits in $\operatorname{Set}_{G U\left(\mathbb{A}^{p, \infty}\right)}^{c o, \alpha}$. Let $\operatorname{Orb}_{G U\left(\mathbb{A}^{p, \infty}\right)}^{c o}$ be the subcategory of orbits. Consider the functor

$$
\begin{aligned}
\operatorname{Orb}_{G U\left(\mathbb{A}^{p, \infty}\right)}^{c o} & \rightarrow\{p \text {-divisible groups }\}, \\
K^{p} & \mapsto\left(S h\left(K^{p}\right), \epsilon \mathbf{A}(u)\right) .
\end{aligned}
$$

Here, $\left(\mathbf{A}, \mathbf{i}, \boldsymbol{\lambda},[\boldsymbol{\eta}]_{K^{p}}\right)$ is the universal tuple on $S h\left(K^{p}\right)$.

Using the isomorphism (10.1.3), functoriality of this correspondence is established as follows: for open compact subgroups $g^{-1} K^{\prime p} g \leq K^{p}$, the map

$$
g_{K^{\prime p}, K^{p}}: S h\left(K^{\prime p}\right) \rightarrow S h\left(K^{p}\right)
$$

classifies the tuple $\left(\mathbf{A}^{\prime}, \mathbf{i}^{\prime}, \boldsymbol{\lambda}^{\prime},\left[\boldsymbol{\eta}^{\prime} g\right]_{K^{p}}\right)$, where $\left(\mathbf{A}^{\prime}, \mathbf{i}^{\prime}, \boldsymbol{\lambda}^{\prime},\left[\boldsymbol{\eta}^{\prime}\right]_{K^{\prime p}}\right)$ is the universal tuple on $S h\left(K^{\prime p}\right)$. In particular, there is a canonical isomorphism of $p$-divisible groups

$$
\alpha_{c a n}: \epsilon \mathbf{A}^{\prime}(u) \stackrel{\cong}{\longrightarrow}\left(g_{K^{\prime}, K^{p}}\right)^{*} \epsilon \mathbf{A}(u)
$$

giving a map

$$
\left(g_{K^{\prime}, K^{p}}, \alpha_{c a n}\right):\left(S h\left(K^{\prime p}\right), \epsilon \mathbf{A}^{\prime}(u)\right) \rightarrow\left(S h\left(K^{p}\right), \epsilon \mathbf{A}(u)\right)
$$

in the category of $p$-divisible groups. The functoriality Theorem 8.1.4 gives an induced map of presheaves of $E_{\infty}$-ring spectra

$$
\left(g_{K^{\prime p}, K^{p}}, \alpha_{c a n}\right)^{*}:\left(g_{K^{\prime p}, K^{p}}\right)^{*} \mathcal{E}\left(K^{p}\right) \rightarrow \mathcal{E}\left(K^{\prime p}\right)
$$

and hence a map

$$
g_{*}: T A F\left(K^{p}\right) \rightarrow T A F\left(K^{\prime p}\right) .
$$

LEMMA 11.1.2. The presheaf TAF $(-)$ on the site $\operatorname{Set}_{G U\left(\mathbb{A}^{p, \infty}\right)}^{c o, \alpha}$ is fibrant.

Proof. Fibrancy is shown to be a local condition in [DHI04], and hence follows from Lemma 8.3.2 and the fibrancy of the presheaves $\mathcal{E}\left(K^{p}\right)$ of Section 8.3.

Construction 10.6.3 associates to the presheaf $T A F(-)$ a fibrant smooth $G U\left(\mathbb{A}^{p, \infty}\right)$ spectrum

$$
V_{G U}=V_{T A F(-)}
$$

By Lemma 10.6.5, for any compact open subgroup $K^{p}<G U\left(\mathbb{A}^{p, \infty}\right)$ we have an equivalence

$$
T A F\left(K^{p}\right) \stackrel{\simeq}{\longrightarrow} V_{G U}^{h K^{p}}
$$

\subsection{Hecke operators}

Let $G=G U\left(\mathbb{A}^{p, \infty}\right)$. Let $\mathcal{M}=\mathcal{M}_{G}^{c o}$ denote the Burnside category. In Section 10.7 we observed that the homotopy fixed points of a smooth $G$-spectrum assembled to yield a Mackey functor in the stable homotopy category. In particular, since the 
spectra $T A F(-)$ are given as homotopy fixed point spectra, our Mackey functor takes the form:

$$
\begin{aligned}
\mathcal{M}^{o p} & \rightarrow \operatorname{Ho}(\mathrm{Sp}) \\
\amalg_{i} G / K_{i} & \mapsto \prod_{i} \operatorname{TAF}\left(K_{i}\right)
\end{aligned}
$$

Let $\mathcal{H}$ denote the Hecke category of $G$. The objects of $\mathcal{H}$ are the same as the objects of $\mathcal{M}$. The morphisms are additively determined by

$$
\operatorname{Hom}_{\mathcal{H}}\left(G / K, G / K^{\prime}\right)=\operatorname{Hom}_{\mathbb{Z}[G]}\left(\mathbb{Z}[G / K], \mathbb{Z}\left[G / K^{\prime}\right]\right) \cong\left(\mathbb{Z}\left[G / K^{\prime}\right]\right)^{K} .
$$

This morphism space is a free abelian group with basis

$$
\left\{T_{[g]}=\sum_{h \in K / K \cap g K^{\prime} g^{-1}} h g K^{\prime}:[g] \in K \backslash G / K^{\prime}\right\} \subset \mathbb{Z}\left[G / K^{\prime}\right]^{K} .
$$

The endomorphisms in $\mathcal{H}$ of $G / K$ give the Hecke algebra of the pair $(G, K)$.

There is a natural functor

$$
F: \mathcal{M} \rightarrow \mathcal{H}
$$

(see [Min99]) which associates to the correspondence

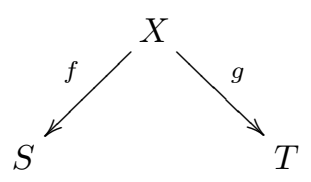

the morphism given by

$$
\begin{aligned}
\mathbb{Z}[S] & \rightarrow \mathbb{Z}[T] \\
s & \mapsto \sum_{x \in f^{-1}(s)} g(x)
\end{aligned}
$$

for elements $s \in S$. The functor $F$ is full, but not faithful. The generators $T_{[g]} \in$ $\operatorname{Hom}_{\mathcal{H}}\left(G / K, G / K^{\prime}\right)$ may be lifted to correspondences

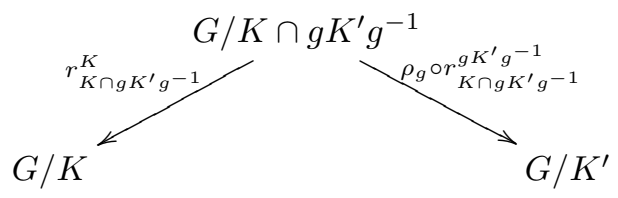

but this does not induce a splitting of $F$.

Definition 11.2.1 (Hecke Operators on $T A F$ ). For $[g] \in K \backslash G / K^{\prime}$ define the Hecke operator

$$
T_{[g]}: T A F\left(K^{\prime}\right) \rightarrow T A F(K)
$$

to be the composite:

$$
T A F\left(K^{\prime}\right) \stackrel{g_{*}}{\longrightarrow} T A F\left(g K^{\prime} g^{-1}\right) \stackrel{\operatorname{Res}_{K \cap g K^{\prime} g^{-1}}^{g K^{\prime} g^{-1}}}{\longrightarrow} T A F\left(K \cap g K^{\prime} g^{-1}\right) \stackrel{\operatorname{Tr}_{K \cap g K^{\prime} g^{-1}}^{K}}{\longrightarrow} T A F(K)
$$


QUESTION 11.2.2. Do these Hecke operators induce a factorization of the following form?

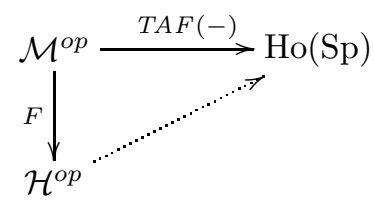

In particular, does this induce a map of rings from the Hecke algebra for $(G, K)$ into the ring of stable cohomology operations on TAF $(K)$ ?

We are unable to resolve this question in general. We can give some partial results.

Proposition 11.2.3. Suppose that for every compact open subgroup $K$ of $G$, and every quasi-coherent sheaf $\mathcal{F}$ on $\operatorname{Sh}(K)_{p}^{\wedge}$, the sheaf cohomology groups

$$
H^{s}\left(S h(K)_{p}^{\wedge}, \mathcal{F}\right)
$$

vanish for $s>0$. Then the Mackey functor TAF(-) factors through the Hecke category.

REMARK 11.2.4. The hypotheses of Proposition 11.2.3 are extremely restrictive. There are morally two ways in which the hypotheses can fail to be satisfied.

(1) The stack $S h(K)$ can fail to have an étale covering space which is an affine scheme.

(2) The stack $S h(K)$ can have stacky points whose automorphism group contains $p$-torsion.

This immediately rules out the case where the algebra $B$ is a division algebra (Theorem 6.6.2(3)). Note that the (non-compactified) moduli stack of elliptic curves $\mathcal{M}_{\text {ell }}$ does satisfy the hypotheses of Proposition 11.2.3 for $p \geq 5$. (See Remark 11.2.7 for more discussion on the modular case.)

Proof of Proposition 11.2.3. Fix a compact open subgroup $K$. The descent spectral sequence (8.3.1)

$$
H^{s}\left(S h(K)_{p}^{\wedge}, \omega^{\otimes t}\right) \Rightarrow \pi_{2 t-s}(T A F(K))
$$

collapses to give an edge isomorphism

$$
\pi_{2 t}(T A F(K)) \stackrel{\cong}{\rightrightarrows} H^{0}\left(S h(K)_{p}^{\wedge}, \omega^{\otimes t}\right) .
$$

By Proposition 8.2.2, our hypotheses guarantee that the spectrum $T A F(K)$ is Landweber exact. Picking a complex orientation for $T A F(K)$, the ring $\pi_{*} T A F(K)$ becomes an $M U_{*}$-algebra, and the natural map

$$
M U_{*} M U \otimes_{M U_{*}} \pi_{*} T A F(K) \rightarrow M U_{*} T A F(K)
$$

is an isomorphism, giving (see [Ada74])

$$
M U_{*} \operatorname{TAF}(K) \cong \pi_{*} \operatorname{TAF}(K)\left[b_{1}, b_{2}, \ldots\right] .
$$

By Landweber exactness, for any pair of compact open subgroups $K, K^{\prime}$, the natural homomorphism

$$
\left[T A F(K), T A F\left(K^{\prime}\right)\right] \rightarrow \operatorname{Hom}_{M U_{*} M U}\left(M U_{*} \operatorname{TAF}(K), M U_{*} T A F\left(K^{\prime}\right)\right)
$$


is an isomorphism [HS99, Cor. 2.17]. Yoshida ([Yos83], [Min99, Thm. 3.7]) proved that a Mackey functor

$$
\mathcal{M}^{o p} \rightarrow \text { Abelian Groups }
$$

factors through the Hecke category if and only if for every $K^{\prime} \leq K$ we have $\operatorname{Tr}_{K^{\prime}}^{K} \operatorname{Res}_{K^{\prime}}^{K}=\left|K: K^{\prime}\right|$. It therefore suffices to prove that the composite

$$
M U_{*} T A F(K) \stackrel{M U_{*} \operatorname{Res}_{K^{\prime}}^{K}}{\longrightarrow} M U_{*} T A F\left(K^{\prime}\right) \stackrel{M U_{*} \operatorname{Tr}_{K^{\prime}}^{K}}{\longrightarrow} M U_{*} T A F(K)
$$

is multiplication by $\left|K: K^{\prime}\right|$.

Recall that for $K^{\prime}$, an open normal subgroup of $K$, the morphism

$$
\operatorname{Sh}\left(K^{\prime}\right) \rightarrow \operatorname{Sh}(K)
$$

is a Galois covering space with Galois group $K / K^{\prime}$ (Theorem 6.6.2). We therefore have

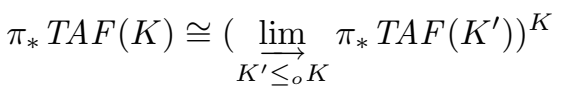

$$
\begin{aligned}
& \cong\left(\pi_{*} V_{G U}\right)^{K} .
\end{aligned}
$$

where the colimit is taken over open subgroups of $K$. We deduce from (11.2.5) that the map

$$
M U_{*}(T A F(K))=M U_{*}\left(V_{G U}^{h K}\right) \rightarrow M U_{*}\left(V_{G U}\right)
$$

is a monomorphism. Consider the following diagram (where $V=V_{G U}$ ). 


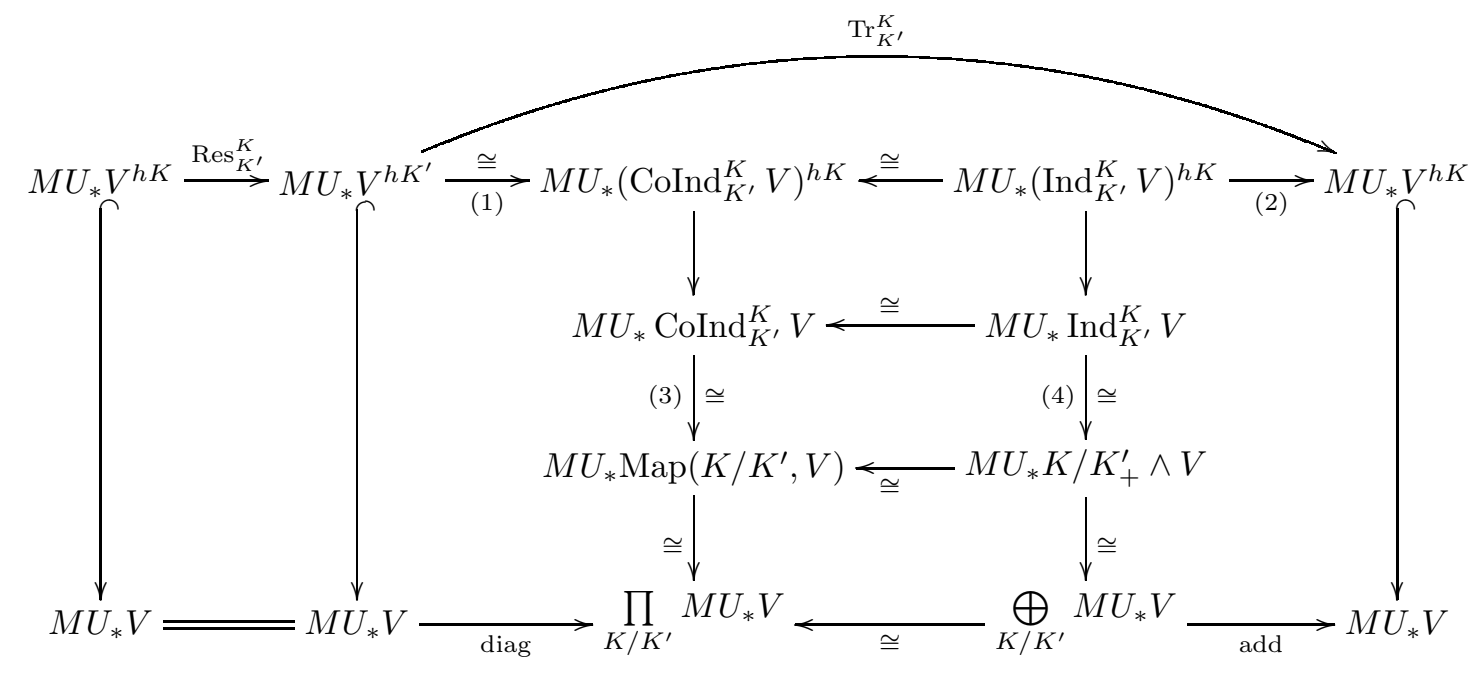


Here (1) is the isomorphism given by Lemma 10.5.2 and (2) is the counit of the adjunction. The isomorphisms (3) and (4) are induced by the canonical $K$-equivariant isomorphisms:

$$
\begin{array}{r}
\operatorname{CoInd}_{K^{\prime}}^{K} V \cong \operatorname{Map}\left(K / K^{\prime}, V\right), \\
\operatorname{Ind}_{K^{\prime}}^{K} V \cong K / K_{+}^{\prime} \wedge V,
\end{array}
$$

where $K$ acts on $\operatorname{Map}\left(K / K^{\prime}, V\right)$ by conjugation and on $K / K_{+}^{\prime} \wedge V$ diagonally. The bottom horizontal composite is multiplication by $\left|K: K^{\prime}\right|$. Since the leftmost and rightmost vertical arrows are monomorphisms, we deduce that the topmost horizontal composite is multiplication by $\left|K: K^{\prime}\right|$.

Precisely the same methods prove the following variant.

Proposition 11.2.6. Suppose that $K$ is a fixed compact open subgroup, and that for every compact open subgroup $K^{\prime}$ of $K$, and every quasi-coherent sheaf $\mathcal{F}$ on $\operatorname{Sh}\left(K^{\prime}\right)_{p}^{\wedge}$, the sheaf cohomology groups

$$
H^{s}\left(\operatorname{Sh}\left(K^{\prime}\right)_{p}^{\wedge}, \mathcal{F}\right)
$$

vanish for $s>0$. Then the Hecke operators induce a homomorphism of rings

$$
\mathbb{Z}[G / K]^{K} \hookrightarrow[T A F(K), T A F(K)] .
$$

Here $\mathbb{Z}[G / K]^{K} \cong \operatorname{Hom}_{\mathbb{Z}[G]}(\mathbb{Z}[G / K], \mathbb{Z}[G / K])$ is the Hecke algebra for $(G, K)$.

REMARK 11.2.7. This is an analog of the work of Baker [Bak90]. Baker proved that for $p \geq 5$, the spectra $T M F_{(p)}$ admit an action of the Hecke algebra for the pair $\left(G L_{2}\left(\mathbb{A}^{p, \infty}\right), G L_{2}\left(\widehat{\mathbb{Z}}^{p}\right)\right)$. The authors do not know if his results extend to the cases where $p=2$ or 3 . These are precisely the cases where the corresponding moduli stack has nontrivial higher sheaf cohomology.

We note that regardless of the outcome of Question 11.2.2, Lemma 10.7.7 implies that the edge homomorphism

$$
\pi_{2 t} \operatorname{TAF}(K) \rightarrow\left(\pi_{2 t} V_{G U}\right)^{K}=H^{0}\left(\operatorname{Sh}(K)_{p}^{\wedge}, \omega^{\otimes t}\right)
$$

commutes with the action of the Hecke operators (as given in Definition 11.2.1). This relates the action of the Hecke operators on $T A F(K)$ to the action of the classical Hecke operators of the corresponding space of automorphic forms. 



\section{CHAPTER 12}

\section{Buildings}

In this chapter we give explicit descriptions for the Bruhat-Tits buildings of the local forms of the group $G U$ and $U$. The buildings are certain finite dimensional contractible simplicial complexes on which the groups $G U\left(\mathbb{Q}_{\ell}\right)$ and $U\left(\mathbb{Q}_{\ell}\right)$ act with compact stabilizers. Our treatment follows closely that of [AN02].

\subsection{Terminology}

A semi-simplicial set is a simplicial set without degeneracies. Thus a semi-simplicial set $\left\{X_{\bullet}, d_{\bullet}\right\}$ consists of a sequence of sets $X_{k}$ of $k$-simplices, with face maps $d_{\bullet}$ : $X_{k} \rightarrow X_{k-1}$ satisfying the simplicial face relations.

A simplicial complex $\left\{X_{\bullet}, \leq\right\}$ consists of a sequence of sets $X_{k}$ of $k$-simplices together with a poset structure on the set $\coprod_{k} X_{k}$ which encodes face containment. Thus a semi-simplicial set is a simplicial complex with a compatible ordering on the vertices of its simplices.

For a finite dimensional simplicial complex $X_{\bullet}$, let $d$ denote the maximal dimension of its simplices. A $d$-simplex in $X$ • is called a chamber. The simplicial complex $X$. is called a chamber complex if every simplex is contained in a chamber, and given any two chambers $a$ and $b$ there is a sequence of chambers

$$
a=a_{0}, a_{1}, \ldots, a_{k}=b
$$

such that for each $i$, the chambers $a_{i}$ and $a_{i-1}$ intersect in a $(d-1)$-dimensional simplex (a panel). A chamber complex is thin if each panel is contained in exactly 2 chambers. A chamber complex is thick if each panel is contained in at least 3 chambers.

For a thin $d$-dimensional chamber complex $X_{\bullet}$, a folding is a morphism of simplicial complexes

$$
f: X_{\bullet} \rightarrow X_{\bullet}
$$

satisfying $f^{2}=f$ for which every chamber in the image of $f$ is in the image of exactly 2 chambers. The complex $X \bullet$ is called a Coxeter complex if, for every pair of chambers $a$ and $b$, there exists a folding $f$ for which $f(a)=b$.

A building $\mathcal{B}$ is a chamber complex which is a union of Coxeter complexes. These subcomplexes are the apartments of $\mathcal{B}$, and are required to satisfy the following two conditions: 
(1) For any pair of chambers, there must exist an apartment containing both of them.

(2) For any pair of apartments $\mathcal{A}_{1}$ and $\mathcal{A}_{2}$, there must be an isomorphism of simplicial complexes

$$
\phi: \mathcal{A}_{1} \rightarrow \mathcal{A}_{2}
$$

such that $\phi$ restricts to the identity on $\mathcal{A}_{1} \cap \mathcal{A}_{2}$.

A building $\mathcal{B}$ is said to be affine if its apartments are tessellations of a Euclidean space by reflection hyperplanes of an irreducible affine reflection group.

REMARK 12.1.1. All of the buildings we shall consider will be affine. The buildings for $S L$ and $U$ will be thick, but the buildings for $G L$ and $G U$ will not be thick.

\subsection{The buildings for $G L$ and $S L$}

Let $K$ be a discretely valued local field with ring of integers $\mathcal{O}$ and uniformizer $\pi$. Let $W$ be a $K$-vector space of dimension $n$.

The building for $G L(W)$. The building $\mathcal{B}(G L(W))$ is the geometric realization of a semi-simplicial set $\mathcal{B}(G L(W))$.. The $k$-simplices are given by sets of lattice chains in $W$ :

$$
\mathcal{B}(G L(W))_{k}=\left\{L_{0}<L_{1}<\cdots<L_{k} \leq \pi^{-1} L_{0}\right\} .
$$

The $i$ th face map is given by deleting the $i$ th lattice from the chain. The group $G L(W)$ acts on the building simplicially by permuting the lattices.

A basis $\mathbf{v}=\left(v_{1}, \ldots, v_{n}\right)$ of $W$ gives rise to a maximal simplex (chamber)

$$
\mathcal{C}(\mathbf{v})=\left(L_{0}(\mathbf{v})<L_{1}(\mathbf{v})<\cdots<L_{n}(\mathbf{v})=\pi^{-1} L_{0}(\mathbf{v})\right)
$$

where

$$
L_{i}(\mathbf{v})=\pi^{-1} \mathcal{O} v_{1} \oplus \cdots \oplus \pi^{-1} \mathcal{O} v_{i} \oplus \mathcal{O} v_{i+1} \oplus \cdots \oplus \mathcal{O} v_{n} .
$$

In particular, the building $\mathcal{B}(G L(W))$ is $n$-dimensional.

The following lemma is clear.

Lemma 12.2.1. The action of $G L(W)$ on $\mathcal{B}(G L(W))$ is transitive on vertices and chambers.

The building for $S L(W)$. Two lattice chains $\left\{L_{i}\right\},\left\{L_{i}^{\prime}\right\}$ are homothetic if there exists an integer $m$ so that for all $i, L_{i}^{\prime}=\pi^{m} L_{i}$.

The building for $S L(W)$ is the quotient of $\mathcal{B}(G L(W))$ given by taking homothety classes of lattice chains. The building $\mathcal{B}(S L(W))$ may be described as the simplicial complex whose $k$-simplices are given by homothety classes of certain lattice chains:

$$
\mathcal{B}(S L(W))_{k}=\left\{\left[L_{0}<L_{1}<\cdots<L_{k}<\pi^{-1} L_{0}\right]\right\} .
$$

The face containment relations of $\mathcal{B}(S L(W))$ are given by chain containment up to homothety. 
The building for $S L(W)$ is $n-1$ dimensional. There is a natural projection of simplicial complexes

$$
\mathcal{B}(G L(W)) \rightarrow \mathcal{B}(S L(W))
$$

and the building $\mathcal{B}(G L(W))$ is homeomorphic to $\mathcal{B}(S L(W)) \times \mathbb{R}$. The $k$-simplices $\left\{L_{i}\right\}$ of $\mathcal{B}(G L(W))$ for which $L_{k}=\pi^{-1} L_{0}$ become degenerate in $\mathcal{B}(S L(W))$.

\subsection{The buildings for $U$ and $G U$}

Suppose now that $K$ is a quadratic extension of $\mathbb{Q}_{\ell}$. Let $(-,-)$ be a non-degenerate hermitian form on $W$.

Totally isotropic subspaces. A vector $v \in W$ is isotropic if $(v, v)=0$. A subspace of $W$ is anisotropic if it contains no non-zero isotropic vectors. A subspace $W^{\prime}$ of $W$ is totally isotropic if $(v, w)=0$ for all $v, w \in W^{\prime}$. A subspace $W^{\prime}$ of $W$ is hyperbolic if it admits a basis $\left(v_{1}, \ldots, v_{2 k}\right)$ such that with respect to this basis the restricted form $\left.(-,-)\right|_{W^{\prime}}$ is given by a matrix

$$
\left[\begin{array}{lll} 
& & * \\
& & \\
& &
\end{array}\right]
$$

where an entry is nonzero if and only if it lies on the reverse diagonal. Such a basis is a hyperbolic basis.

The Witt index $r$ of $(-,-)$ is the dimension of the maximally totally isotropic subspace of $W$. The dimension of a maximal hyperbolic subspace $V$ of $W$ is $2 r$, and the subspace $V^{\perp}$ is anisotropic. The dimensions of the buildings for $G U(W)$ and $U(W)$ are functions of the Witt index.

Recall from Chapter 5 that isometry classes of quadratic hermitian forms are classified by their discriminant disc in $\mathbb{Q}_{\ell}^{\times} / N\left(K^{\times}\right)$. Let $a \in \mathbb{Q}_{\ell}^{\times}$be a generator of $\mathbb{Q}_{\ell}^{\times} / N\left(K^{\times}\right)$. The following lemma is easily deduced.

Lemma 12.3.1. There exists a basis so that the form $(-,-)$ is given by the matrices in the following table. 


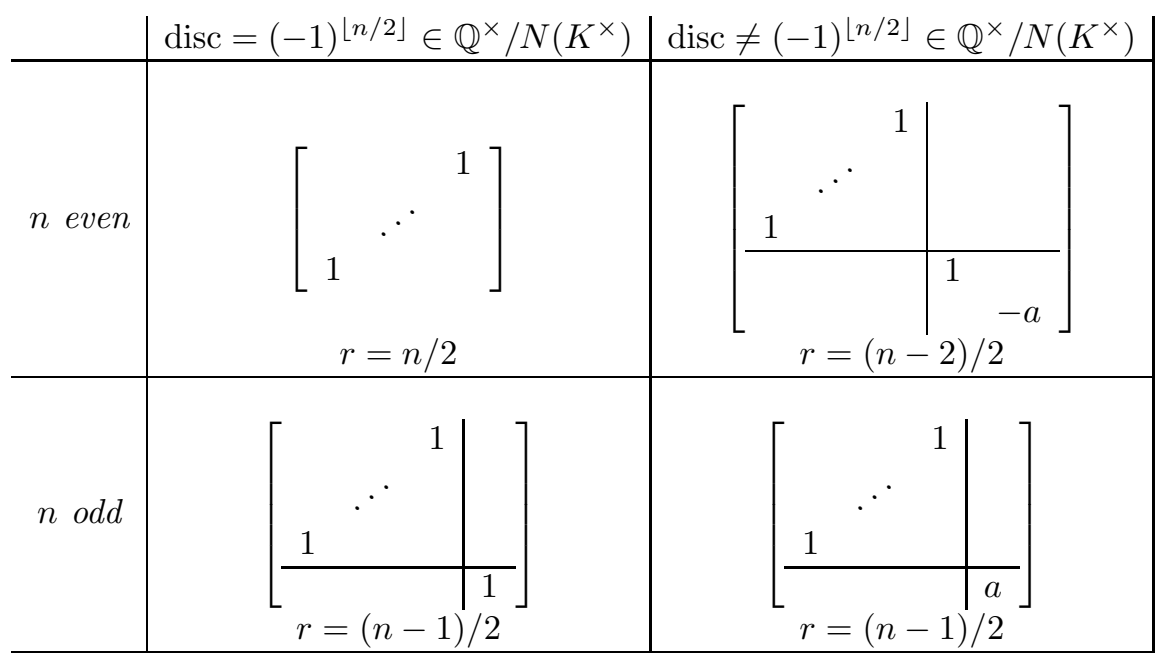

The building for $U$. Given an $\mathcal{O}$-lattice $L$ of $W$, the dual lattice $L^{\#} \subset W$ is the $\mathcal{O}$-lattice given by

$$
L^{\#}=\{w \in W:(w, L) \subseteq \mathcal{O}\} .
$$

The dual lattice construction has the following properties:

(1) $L^{\# \#}=L$,

(2) if $L_{0}<L_{1}$, then $L_{1}^{\#}<L_{0}^{\#}$,

(3) $(\pi L)^{\#}=\pi^{-1} L^{\#}$.

Thus, there is an induced involution

$$
\iota: \mathcal{B}(S L(W)) \rightarrow \mathcal{B}(S L(W)) .
$$

The building $\mathcal{B}(U(W)) \subseteq \mathcal{B}(S L(W))$ consists of the fixed points of this involution. Since the involution $\iota$ does not necessarily restrict to the identity on invariant chambers, the subspace $\mathcal{B}(U(W))$ is not necessarily a simplicial subcomplex.

However, the building $\mathcal{B}(U)$ does admit the structure of a semi-simplicial set whose simplices are self-dual lattice chains. The $k$-simplices are given by

$$
\mathcal{B}(U(W))_{k}=\left\{L_{0}<L_{1}<\cdots<L_{k} \leq L_{k}^{\#}<\cdots<L_{0}^{\#} \leq \pi^{-1} L_{0}\right\} .
$$

The face maps $d_{i}$ are obtained by deletion of the lattices $L_{i}$ and $L_{i}^{\#}$ from the chain. In particular, the vertices are given by lattices $L$ satisfying $L \leq L^{\#} \leq \pi^{-1} L$. We shall say that such lattices are preferred. Thus the $k$-simplices of $\mathcal{B}(U(W))$ are given by chains of preferred lattices:

$$
\mathcal{B}(U(W))_{k}=\left\{L_{0}<L_{1}<\cdots<L_{k}<\pi^{-1} L_{0}: L_{i} \text { are preferred }\right\} .
$$

Preferred lattices satisfy the following properties.

(1) There is at most one preferred representative in a homothety lattice class.

(2) If $L$ is preferred, then either $L=L^{\#}$, or $L^{\#}$ is not preferred. 
These properties allow one to relate the simplicial description of $\mathcal{B}(U(W))$ with the geometric fixed points of the involution $\iota$ on $\mathcal{B}(S L(W))$. A combinatorial vertex corresponding to a preferred lattice $L$ corresponds to the midpoint of the edge joining $[L]$ and $\left[L^{\#}\right]$.

It is easily checked that for $g \in G L(W)$, one has $(g(L))^{\#}=g^{-\iota}\left(L^{\#}\right)$. Therefore the building $\mathcal{B}(U(W))$ admits a simplicial action by $U(W)$. Let $G U^{1}(W)$ be the subgroup

$$
G U^{1}(W)=\operatorname{ker}\left(G U(W) \stackrel{\nu}{\rightarrow} \mathbb{Q}_{\ell}^{\times} \stackrel{\nu_{\ell}}{\longrightarrow} \mathbb{Z}\right)
$$

of similitudes of $W$ with similitude norm of valuation 0 . Then the following lemma is immediate.

Lemma 12.3.2. The action of $U(W)$ on $\mathcal{B}(U(W))$ extends to an action of $G U^{1}(W)$.

In order to get an explicit description of the chambers of $\mathcal{B}(U(W))$ we recall the following lemma of [AN02, Sec. 5].

Lemma 12.3.3. Suppose that the residue characteristic $K$ does not equal 2. Let $W^{\prime}$ be an anisotropic subspace of $W$. Then there is a unique preferred lattice $X$ contained in $W^{\prime}$. This lattice is given by

$$
X=\left\{w \in W^{\prime}:(w, w) \in \mathcal{O}\right\} .
$$

Let $\mathbf{v}=\left(v_{1}, \ldots, v_{2 r}\right)$ be a hyperbolic basis of a maximal hyperbolic subspace $V$ of $W$ such that $\left(v_{i}, v_{2 r+1-i}\right)=1$. We shall say that a hyperbolic basis with this property is normalized, and clearly every hyperbolic basis can be normalized. Let $X$ be the unique preferred lattice in the anisotropic subspace $V^{\perp}$. Then we may associate to $\mathbf{v}$ a chamber

$$
\mathcal{C}(\mathbf{v})=\left(L_{0}(\mathbf{v})<\cdots<L_{r}(\mathbf{v}) \leq L_{r}^{\#}(\mathbf{v})<\cdots<L_{0}^{\#}(\mathbf{v}) \leq \pi^{-1} L_{0}(\mathbf{v})\right)
$$

where

$$
L_{i}(\mathbf{v})=\pi \mathcal{O} v_{1}+\cdots \pi \mathcal{O} v_{r-i}+\mathcal{O} v_{r-i+1}+\cdots \mathcal{O} v_{2 r}+X .
$$

In particular the building $\mathcal{B}(U(W))$ has dimension $r$.

Unlike the building for $S L(W)$, the group $U(W)$ does not act transitively on vertices. However, every chamber of $\mathcal{B}(U(W))$ can be shown to take the form $\mathcal{C}(\mathbf{v})$ for some normalized hyperbolic basis $\mathbf{v}$. Witt's theorem and Lemma 12.3.3 may then be used to derive the following lemma.

Lemma 12.3.4. The group $U(W)$ acts transitively on the set of chambers of the building $\mathcal{B}(U(W))$.

Lemma 12.3.5. The stabilizers of the action of $U(W)$ and $G U^{1}(W)$ on $\mathcal{B}(U(W))$ are all compact and open.

Proof. We give the proof for $U(W)$; the argument for $G U^{1}(W)$ is identical. Let $x=(L)$ be a vertex of $\mathcal{B}(U(W))$ given by a preferred lattice $L$, and let $K_{x} \subset$ $U(W)$ be its stabilizer. Then $K_{x}$ is given by the intersection $G L(L) \cap U(W)$ in $G L(W)$. Since $U(W)$ is a closed subgroup of $G L(W)$ and $G L(L)$ is compact and open, the subgroup $K_{x}$ is compact and open in $U(W)$. Since the action is simplicial, it follows that all of the stabilizers of simplices in $\mathcal{B}(U(W))$ are compact and open. 
The building for $G U(W)$. The group $G U(W)$ does not preserve the class of preferred lattices, but we do have the following lemma.

LEMma 12.3.6. Let $g$ be an element of $G U(W)$ with similitude norm $\nu(g)$ of valuation $k$. Suppose that $L$ is homothetic to a preferred lattice. Then $(g(L))^{k \#}$ is homothetic to a preferred lattice.

Proof. Suppose that $L=\pi^{b} \bar{L}$ where $\bar{L}$ is a preferred lattice. Observe that we have

$$
g\left(\bar{L}^{\#}\right)=\left(g^{-\iota}(\bar{L})\right)^{\#}=\left(\nu^{-1} g(\bar{L})\right)^{\#}=\pi^{k}(g(\bar{L}))^{\#} .
$$

Suppose that $k=2 a$ is even. Then the element $g_{1}=\pi^{-a} g$ has similitude norm of valuation zero, and $g_{1}(\bar{L})$ is preferred. The lattice $g(L)$ is homothetic to $g_{1}(\bar{L})$.

Suppose that $k=2 a-1$ is odd. The element $g_{1}=\pi^{-a} g$ has similitude norm of valuation -1 . Applying $g_{1}$ to the chain $\pi L^{\#} \leq L \leq L^{\#}$, and using the fact that $\left(g_{1}(\bar{L})\right)^{\#}=\pi g_{1}\left(\bar{L}^{\#}\right)$, we see that $\left(g_{1}(\bar{L})\right)^{\#}$ is preferred. The lattice $(g(L))^{\#}$ is therefore homothetic to a preferred lattice.

The action of $U(W)$ on the building $\mathcal{B}(U(W))$ can be extended to an action of the group $G U(W)$. The action is determined by its effect on vertices, where we regard the vertices as preferred homothety classes of lattices. The action is given by

$$
g \cdot[L]= \begin{cases}{[g(L)]} & \text { if } \nu(g) \text { has even valuation, } \\ {\left[(g(L))^{\#}\right]} & \text { if } \nu(g) \text { has odd valuation }\end{cases}
$$

Lemma 12.3.6 implies that $g \cdot[L]$ is a preferred homothety class. This action is not associative on lattices, but is associative on homothety classes. This is an action of simplicial complexes, but does not preserve the ordering on the vertices.

The building $\mathcal{B}(S L(W))$ carries a natural action by $G U(W)$ through the natural action of $G L(W)$. We have the following lemma.

Lemma 12.3.7. The inclusion of the fixed point space $j: \mathcal{B}(U(W)) \hookrightarrow \mathcal{B}(S L(W))$ is $G U(W)$-equivariant.

PROOF. It suffices to check equivariance on vertices. Recall that for a preferred lattice $L$, the inclusion $j$ maps $[L]$ to the midpoint $\operatorname{mid}\left([L],\left[L^{\#}\right]\right)$. Suppose that $g$ is an element of $G U(W)$, with similitude norm of valuation $k$. Then we have

$$
\begin{aligned}
j(g \cdot[L]) & =j\left(\left[(g(L))^{k \#}\right]\right) \\
& =\operatorname{mid}\left(\left[(g(L))^{k \#}\right],\left[(g(L))^{(k+1) \#}\right]\right) \\
& =\operatorname{mid}\left([g(L)],\left[(g(L))^{\#}\right]\right) \\
& =g \cdot j([L]) .
\end{aligned}
$$


We define $\mathcal{B}(G U(W))$ by the following $G U(W)$-equivariant pullback.

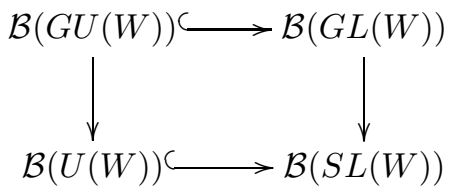

We see that $\mathcal{B}(G U(W))$ is homeomorphic to $\mathcal{B}(U(W)) \times \mathbb{R}$. In particular, the dimension of $\mathcal{B}(G U(W))$ is $r+1$.

Since $G U(W)$ is a closed subgroup of $G L(W)$, and the stabilizer in $G L(W)$ of every point of $\mathcal{B}(G L(W))$ is open and compact, we have the following lemma.

Lemma 12.3.8. The stabilizer in $G U(W)$ of every point of $\mathcal{B}(G U(W))$ is open and compact.

REMARK 12.3.9. The buildings $\mathcal{B}(G U(W))$ and $\mathcal{B}(G L(W)$ ) are easily seen (using a simplicial prism decomposition) to be equivariantly homeomorphic to the products

$$
\begin{aligned}
\mathcal{B}(G U(W)) & \approx \mathcal{B}(U(W)) \times \mathbb{R}_{\nu}, \\
\mathcal{B}(G L(W)) & \approx \mathcal{B}(S L(W)) \times \mathbb{R}_{\mathrm{det}} .
\end{aligned}
$$

Here, $\mathbb{R}_{\nu}$ is the similitude line, with $G U(W)$ action $g(x)=v_{\pi}(\nu(g)) \cdot x$, and $\mathbb{R}_{\text {det }}$ is the determinant line, with $G L(W)$ action $g(x)=v_{\pi}(\operatorname{det}(g)) \cdot x$. The embedding of $\mathcal{B}(G U(W))$ into $\mathcal{B}(G L(W))$ is given by the embedding of $\mathcal{B}(U(W))$ into $\mathcal{B}(S L(W))$, together with the $G U(W)$-equivariant homeomorphism

$$
\begin{aligned}
& \mathbb{R}_{\nu} \stackrel{\approx}{\stackrel{\sim}{\rightarrow}} \mathbb{R}_{\mathrm{det}}, \\
& x \mapsto n / f \cdot x .
\end{aligned}
$$

Here, $f$ is the residue field degree of $K$ over $\mathbb{Q}_{\ell}$. 

CHAPTER 13

\section{Hypercohomology of adele groups}

\subsection{Definition of $Q_{G U}$ and $Q_{U}$}

Lemma 10.6.5 has the following corollary.

Corollary 13.1.1. For compact open $K^{p} \leq G U\left(\mathbb{A}^{p, \infty}\right)$ there are equivalences

$$
\operatorname{TAF}\left(K^{p}\right) \simeq V_{G U}^{h K^{p}} .
$$

Definition 13.1.2. Let $K^{p}=\prod_{\ell \neq p} K_{\ell}$ be a compact open subgroup of $G U\left(\mathbb{A}^{p, \infty}\right)$, and let $S$ be a set of primes not containing $p$. Let $K^{p, S}$ be the product

$$
K^{p, S}=\prod_{\ell \notin S \cup\{p\}} K_{\ell}
$$

(1) Define $Q_{G U}\left(K^{p, S}\right)$ to be the homotopy fixed point spectrum

$$
Q_{G U}\left(K^{p, S}\right)=V_{G U}^{h K^{p, S}}
$$

where

$$
K_{+}^{p, S}:=G U\left(\mathbb{A}_{S}\right) K^{p, S} .
$$

If $S$ consists of all primes different from $\ell$, we denote this spectrum $Q_{G U}$.

(2) Let $G U^{1}\left(\mathbb{A}_{S}\right)$ be the open subgroup

$$
G U^{1}\left(\mathbb{A}_{S}\right)=\operatorname{ker}\left(G U\left(\mathbb{A}_{S}\right) \stackrel{\nu}{\rightarrow} \prod_{\ell \in S}^{\prime} \mathbb{Q}_{\ell}^{\times} \stackrel{\nu_{\ell}}{\longrightarrow} \prod_{\ell \in S} \mathbb{Z}\right)
$$

of similitudes $g$ whose similitude norm $\nu(g)$ has valuation 0 at every place in $S$. Define $Q_{U}\left(K^{p, S}\right)$ to be the homotopy fixed point spectrum

$$
Q_{U}\left(K^{p, S}\right)=V_{G U}^{h K_{1,+}^{p, S}},
$$

where

$$
K_{1,+}^{p, S}:=G U^{1}\left(\mathbb{A}_{S}\right) K^{p, S} .
$$

If $S$ consists of all primes different from $\ell$, we denote this spectrum $Q_{U}$.

\subsection{The semi-cosimplicial resolution}

Fix a prime $\ell \neq p$ and a compact open subgroup $K^{p, \ell} \subset G U\left(\mathbb{A}^{p, \ell, \infty}\right)$. We shall consider two cases simultaneously: 


\begin{tabular}{c|c|c} 
& Case I & Case II \\
\hline$G_{\ell}=$ & $G U\left(\mathbb{Q}_{\ell}\right)$ & $G U^{1}\left(\mathbb{Q}_{\ell}\right)$ \\
$\mathcal{B}=$ & $\mathcal{B}\left(G U\left(\mathbb{Q}_{\ell}\right)\right)$ & $\mathcal{B}\left(U\left(\mathbb{Q}_{\ell}\right)\right)$ \\
$Q=$ & $Q_{G U}\left(K^{p, \ell}\right)$ & $Q_{U}\left(K^{p, \ell}\right)$
\end{tabular}

In either case, $\mathcal{B}=\left|\mathcal{B}_{\bullet}\right|$ is the realization of a finite dimensional contractible semi-simplicial set $\mathcal{B}_{\bullet}$, and the group $G_{\ell}$ acts on $\mathcal{B}$ simplicially with compact open stabilizers. Throughout this section, we will let $\mathcal{B}_{\bullet}^{\prime}$ be the simplicial set generated by the semi-simplicial set $\mathcal{B}$. by including degeneracies.

Let $G$ denote the open subgroup

$$
G=G_{\ell} K^{p, \ell} \subset G U\left(\mathbb{A}^{p, \infty}\right)
$$

so that $Q=V_{G U}^{h G}$. The action of the group $G_{\ell}$ on $\mathcal{B}$ naturally extends to an action of the group $G$, where we simply let the factors $K_{\ell^{\prime}}$ act trivially for $\ell^{\prime} \neq p, \ell$. The stabilizers of this action are still compact and open.

The canonical contracting homotopy. We recall some of the material from [Gar97], in particular Section 13.7. These apartments of $\mathcal{B}$ possess canonical metrics, and simplicial automorphisms of these are isometries. The building $\mathcal{B}$ inherits a metric as follows: for $x, y \in \mathcal{B}$, let $\mathcal{A}$ be an apartment containing $x$ and $y$. The distance between $x$ and $y$ is equal to the distance taken in the apartment $\mathcal{A}$ with respect to its canonical metric. Since the group $G$ acts simplicially on $\mathcal{B}$, and sends apartments to apartments, $G$ acts by isometries on $\mathcal{B}$.

Any two points $x, y \in \mathcal{B}$, are joined by a unique geodesic

$$
\gamma_{x, y}: I \rightarrow \mathcal{B}
$$

Let $\mathcal{A}$ be an apartment of $\mathcal{B}$ which contains both $x$ and $y$. Then the geodesic $\gamma_{x, y}$ is given by the affine combination

$$
\gamma_{x, y}(t)=(1-t) x+t y
$$

in the affine space $\mathcal{A}$.

A contracting homotopy for $\mathcal{B}$ is very simple to describe [Gar97, Sec. 14.4]. Fix a point $x_{0}$ of $\mathcal{B}$. A contracting homotopy

$$
H: \mathcal{B} \times I \rightarrow \mathcal{B}
$$

is given by

$$
H_{t}(x)=\gamma_{x, x_{0}}(t)
$$

REMARK 13.2.2. The argument [Gar97, Sec. 14.4] that the contracting homotopy $H$ is continuous applies to thick buildings. While the building $\mathcal{B}\left(U\left(\mathbb{Q}_{\ell}\right)\right)$ is thick if $\ell$ does not split in $F$, the building $\mathcal{B}\left(U\left(\mathbb{Q}_{\ell}\right)\right)$ in the split case, as well as the building $\mathcal{B}\left(G U\left(\mathbb{Q}_{\ell}\right)\right)$ in either case, is not thick. However, because these other buildings are 
products of thick buildings with an affine space, the continuity of $H$ is easily seen to extend to these cases.

A technical lemma. Let $E$ be a smooth $G$-spectrum, and suppose that $X$ is a simplicial $G$-set. For an open subgroup $U \leq G$, define $\operatorname{Map}_{U}(X, E)$ to be the spectrum whose $i$ th space is the simplicial mapping space of $U$-equivariant maps:

$$
\underline{\operatorname{Map}}_{U}(X, E)_{i}=\underline{\operatorname{Map}}_{U}\left(X, E_{i}\right) .
$$

Define a smooth $G$-spectrum

$$
\underline{\operatorname{Map}}(X, E)^{s m}:=\lim _{U \leq_{o} G} \underline{\operatorname{Map}}_{U}(X, E)
$$

(where the colimit is taken over open subgroups of $G$ ). The group $G$ acts on the maps in the colimit by conjugation. In this section we will prove the following technical lemma.

Lemma 13.2.3. The map of smooth $G$-spectra

$$
E \rightarrow \underline{\operatorname{Map}}\left(\mathcal{B}_{\bullet}^{\prime}, E\right)^{s m}
$$

induced by the map $\mathcal{B}_{\bullet}^{\prime} \rightarrow *$ is an equivalence.

The key point to proving Lemma 13.2.3 is the following topological lemma.

Lemma 13.2.4. Suppose that $X$ is a topological space with a smooth $G$-action. Then the inclusion of the constant maps induces a natural inclusion

$$
X \rightarrow \operatorname{Map}(\mathcal{B}, X)^{s m}:=\underset{U \leq_{\circ} G}{\lim _{\longrightarrow}} \operatorname{Map}_{U}(\mathcal{B}, X)
$$

which is the inclusion of a deformation retract. Here, $\operatorname{Map}_{U}(\mathcal{B}, X)$ is given the subspace topology with respect to the mapping space $\operatorname{Map}(\mathcal{B}, X)$, and $\operatorname{Map}(\mathcal{B}, X)^{\text {sm }}$ is given the topology of the union of the spaces $\operatorname{Map}_{U}(\mathcal{B}, X)$.

Proof. Fix a point $x_{0}$ in $\mathcal{B}$ with compact open stabilizer $K_{x_{0}} \leq G$. Let $H$ be the contracting homotopy defined by (13.2.1). Then $H$ induces a deformation retract

$$
H^{\prime}: \operatorname{Map}(\mathcal{B}, X) \times I \rightarrow \operatorname{Map}(\mathcal{B}, X)
$$

given by

$$
H_{t}^{\prime}(f)(x)=f\left(H_{t}(x)\right)=f\left(\gamma_{x, x_{0}}(t)\right)
$$

where $\gamma_{x, x_{0}}$ is the unique geodesic from $x$ to $x_{0}$. We claim that for each open subgroup $U$ of $G, H^{\prime}$ restricts to give a map

$$
H^{\prime}: \operatorname{Map}_{U}(\mathcal{B}, X) \times I \rightarrow \operatorname{Map}_{U \cap K_{x_{0}}}(\mathcal{B}, X) .
$$

Indeed, let $f$ be an element of $\operatorname{Map}_{U}(\mathcal{B}, X)$. Let $g$ be an element of $U \cap K_{x_{0}}$. Since $G$ acts by isometries, the induced map

$$
g: \mathcal{B} \rightarrow \mathcal{B}
$$


sends geodesics to geodesics. Hence we have

$$
\begin{aligned}
g\left(H_{t}^{\prime}(f)(x)\right) & =g\left(f\left(\gamma_{x, x_{0}}(t)\right)\right) \\
& =f\left(g \gamma_{x, x_{0}}(t)\right) \\
& =f\left(\gamma_{g x, g x_{0}}(t)\right) \\
& =f\left(\gamma_{g x, x_{0}}(t)\right) \\
& =H_{t}^{\prime}(f)(g x) .
\end{aligned}
$$

We have shown that $H_{t}^{\prime}(f)$ is $U \cap K_{x_{0}}$-equivariant.

By taking the colimit of the maps $H_{t}^{\prime}$ of (13.2.5), we obtain a deformation retract

$$
H^{\prime}: \operatorname{Map}(\mathcal{B}, X)^{s m} \times I \rightarrow \operatorname{Map}(\mathcal{B}, X)^{s m} .
$$

We now explain how, using the geometric realization and singular functors, the topological result of Lemma 13.2.4 gives an analogous simplicial result. The category $s$ Set of simplicial sets and the category Top of topological spaces are Quillen equivalent by adjoint functors $(|-|, S)$

$$
|-|: s \text { Set } \leftrightarrows \text { Top : } S
$$

where $S(-)$ is the singular complex functor and $|-|$ is geometric realization. The geometric realization of a simplicial smooth $G$-set is a $G$-space with smooth $G$ action. Since the realization of the $k$-simplex $\left|\Delta^{k}\right|$ is compact, it is easily verified that the singular chains on a $G$-space with smooth $G$-action is a simplicial smooth $G$-set.

Lemma 13.2.6. Suppose that $X$ is a fibrant simplicial smooth $G$-set. Then $S|X|$, the singular complex of the geometric realization of $X$, is also fibrant.

Proof. By Lemma 10.2.7, we must verify that for every open subgroup $H \leq G$, the $H$-fixed points of $S|X|$ is a Kan complex, and that $S|X|$ satisfies homotopy descent with respect to hypercovers of $G / H$. Since both the functors $S(-)$ and $|-|$ both preserve fixed points, we see that $(S|X|)^{H}=S\left|X^{H}\right|$ is a Kan complex. For a hypercover $\left\{G / U_{\alpha, \bullet}\right\}_{\alpha \in I_{\bullet}}$, we must verify that the map

$$
(S|X|)^{H} \rightarrow \operatorname{holim}_{\Delta} \prod_{\alpha \in I_{\bullet}}(S|X|)^{U_{\alpha} \bullet}
$$

is a weak equivalence. The functors $S(-)$ and $|-|$ commute with fixed points, $S(-)$ commutes with arbitrary products and totalization, and $|-|$ commutes (up to homotopy equivalence) with arbitrary products of Kan complexes, so we need the map

$$
S\left|X^{H}\right| \rightarrow S \underset{\Delta}{\operatorname{holim}}\left|\prod_{\alpha \in I_{\bullet}} X^{U_{\alpha, \bullet}}\right|
$$

to be a weak equivalence. It suffices to show that the map

$$
\left|X^{H}\right| \rightarrow \underset{\Delta}{\operatorname{holim}}\left|\prod_{\alpha \in I_{\bullet}} X^{U_{\alpha}, \bullet}\right|
$$


is a weak equivalence, or equivalently, since $(|-|, S(-))$ form Quillen equivalence, that the map

$$
X^{H} \rightarrow S \underset{\Delta}{\operatorname{holim}}\left|\prod_{\alpha \in I_{\bullet}} X^{U_{\alpha}, \bullet} \stackrel{\simeq}{\rightarrow} \operatorname{holim}_{\Delta} S\right| \prod_{\alpha \in I_{\bullet}} X^{U_{\alpha}, \bullet} \mid
$$

is a weak equivalence. This follows from the fact that the map

$$
\prod_{\alpha \in I_{\bullet}} X^{U_{\alpha} \bullet} \rightarrow S\left|\prod_{\alpha \in I_{\bullet}} X^{U_{\alpha, \bullet}}\right|
$$

is a level-wise weak equivalence of cosimplicial Kan complexes, and that the map

$$
X^{H} \rightarrow \underset{\Delta}{\operatorname{holim}} \prod_{\alpha \in I_{\bullet}} X^{U_{\alpha}, \bullet}
$$

is a weak equivalence, since $X$ is fibrant.

Lemma 13.2.3 follows immediately from the following lemma.

Lemma 13.2.7. Let $X$ be a simplicial smooth $G$-set. The natural map

$$
X \rightarrow \underline{\operatorname{Map}}\left(\mathcal{B}_{\bullet}^{\prime}, X\right)^{s m}
$$

is a weak equivalence of simplicial sets.

Proof. By Lemma 13.2.4, the map

$$
|X| \stackrel{\simeq}{\longrightarrow} \operatorname{Map}(\mathcal{B},|X|)^{s m}
$$

is an equivalence. Since geometric realization is the left adjoint of a Quillen equivalence, we see that the adjoint

$$
X \stackrel{\simeq}{\longrightarrow} S \operatorname{Map}(\mathcal{B},|X|)^{s m}
$$

is an equivalence. We have the following sequence of isomorphisms:

$$
\begin{aligned}
& S \operatorname{Map}\left(\left|\mathcal{B}_{\bullet}^{\prime}\right|,|X|\right)^{s m}=S \underset{U \leq_{o} G}{\lim _{\longrightarrow}} \operatorname{Map}_{U}\left(\left|\mathcal{B}_{\bullet}^{\prime}\right|,|X|\right) \\
& \cong \operatorname{Map}^{\operatorname{Top}}\left(\left|\Delta^{\bullet}\right|, \underset{U \leq_{\circ} G}{\lim _{\longrightarrow}} \operatorname{Map}_{U}\left(\left|\mathcal{B}_{\bullet}^{\prime}\right|,|X|\right)\right) \\
& \cong \lim _{U \leq_{o} G} \operatorname{Map}^{\operatorname{Top}}\left(\left|\Delta^{\bullet}\right|, \operatorname{Map}_{U}\left(\left|\mathcal{B}_{\bullet}^{\prime}\right|,|X|\right)\right) \\
& \cong \lim _{U \leq_{\circ} G} \operatorname{Map}_{U}^{\operatorname{Top}}\left(\left|\Delta^{\bullet}\right| \times\left|\mathcal{B}_{\bullet}^{\prime}\right|,|X|\right) \\
& \cong \lim _{U \leq_{o} G} \operatorname{Map}_{U}^{\operatorname{Top}}\left(\left|\Delta^{\bullet} \times \mathcal{B}_{\bullet}^{\prime}\right|,|X|\right) \\
& \cong \lim _{U \leq_{0} G} \operatorname{Map}_{U}^{s \operatorname{Set}}\left(\Delta^{\bullet} \times \mathcal{B}_{\bullet}^{\prime}, S|X|\right) \\
& =\underline{\operatorname{Map}}\left(\mathcal{B}_{\bullet}^{\prime}, S|X|\right)^{s m} \text {. }
\end{aligned}
$$


This isomorphism fits into the following commutative diagram.

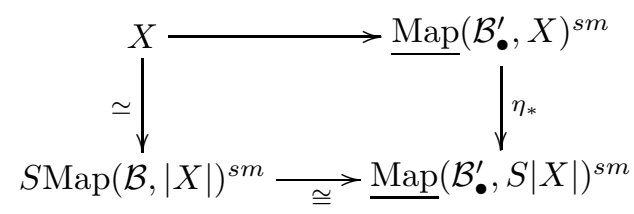

We just need to verify that the map $\eta_{*}$ is a weak equivalence. For any simplicial smooth $G$-set $Z$, the functor $-\times Z$ preserves weak equivalences and cofibrations. Therefore, the functors $\left(-\times Z, \operatorname{Map}(Z,-)^{s m}\right)$ form a Quillen adjoint pair on $s \operatorname{Set}_{G}^{s m}$. In particular, the functor $\overline{\operatorname{Map}}\left(\mathcal{B}_{\bullet}^{\prime},-\right)^{s m}$ preserves weak equivalences between fibrant simplicial smooth $G$-sets. By Lemma 13.2.6, the map

$$
\eta: X \rightarrow S|X|
$$

is a weak equivalence between fibrant simplicial smooth $G$-sets.

REMARK 13.2.8. The authors do not know a purely simplicial argument to prove Lemma 13.2.3. Clearly, much of the work in this section could be eliminated by working with spectra of topological spaces with smooth $G$-action as opposed to spectra of simplicial smooth $G$-sets. Our reason for choosing to work simplicially is that we are simply unaware of a treatment of local model structures on categories of sheaves of topological spaces in the literature.

The semi-cosimplicial resolution. Recall from the beginning of this section that $Q$ is the hypercohomology spectrum $Q_{G U}\left(K^{p, \ell}\right)$ or $Q_{U}\left(K^{p, \ell}\right)$. We state our main theorem describing a finite semi-cosimplicial resolution of $Q$.

TheOREM 13.2.9. There is a semi-cosimplicial spectrum $Q^{\bullet}$ of length $d=\operatorname{dim} \mathcal{B}$ whose sth term $(0 \leq s \leq d)$ is given by

$$
Q^{s}=\prod_{[\sigma]} T A F(K(\sigma)) .
$$

The product ranges over $G_{\ell}$ orbits of s-simplices $[\sigma]$ in the building $\mathcal{B}$. The groups $K(\sigma)$ are given by

$$
K(\sigma)=K^{p, \ell} K_{\ell}(\sigma)
$$

where $K_{\ell}(\sigma)$ is the subgroup of $G_{\ell}$ which stabilizes $\sigma$. There is an equivalence

$$
Q \simeq \operatorname{holim} Q^{\bullet} .
$$

Proof. By Lemma 13.2.3, the map

$$
r: V_{G U} \rightarrow \underline{\operatorname{Map}}\left(\mathcal{B}_{\bullet}^{\prime}, V_{G U}\right)^{s m}
$$

is an equivalence of smooth $G$-spectra. The functor

$$
\underline{\operatorname{Map}}\left(\mathcal{B}_{\bullet}^{\prime},-\right)^{s m}: \mathrm{Sp}_{G}^{s m} \rightarrow \operatorname{Sp}_{G}^{s m}
$$


is right Quillen adjoint to the functor $-\wedge\left(\mathcal{B}_{\bullet}^{\prime}\right)_{+}$. Therefore, since $V_{G U}$ is a fibrant smooth $G$-spectrum, the spectrum $\operatorname{Map}\left(\mathcal{B}_{\bullet}^{\prime}, V_{G U}\right)^{s m}$ is a fibrant smooth $G$ spectrum. We have the following sequence of $G$-equivariant isomorphisms.

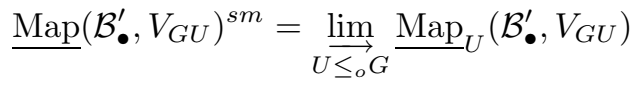

$$
\begin{aligned}
& \cong \lim _{U \leq_{\circ} G} \operatorname{holim} \operatorname{Map}_{U}\left(\mathcal{B}_{\bullet}^{\prime}, V_{G U}\right) \\
& \cong \lim _{U \leq_{\circ} G} \operatorname{holim} \operatorname{Map}_{U}\left(\mathcal{B}_{\bullet}, V_{G U}\right) \\
& \cong \operatorname{holim} \lim _{U \leq_{o} G} \operatorname{Map}_{U}\left(\mathcal{B}_{\bullet}, V_{G U}\right) \\
& \cong \operatorname{holim} \operatorname{Map}\left(\mathcal{B}_{\bullet}, V_{G U}\right)^{s m} \text {. }
\end{aligned}
$$

Here, we were able to commute the homotopy limit past the colimit because it is the homotopy limit of a finite length semi-cosimplicial spectrum. Taking $G$-fixed points of both sides, we have a map

$$
r^{G}: V_{G U}^{G} \rightarrow\left(\operatorname{holim} \operatorname{Map}\left(\mathcal{B}_{\bullet}, V_{G U}\right)^{s m}\right)^{G} .
$$

The map $r^{G}$ is a weak equivalence because $r$ is a weak equivalence between fibrant smooth $G$-spectra. There are isomorphisms

$$
\begin{aligned}
\left(\operatorname{holim} \operatorname{Map}\left(\mathcal{B}_{\bullet}, V_{G U}\right)^{s m}\right)^{G} & \cong \operatorname{holim} \operatorname{Map}_{G}\left(\mathcal{B}_{\bullet}, V_{G U}\right) \\
& \cong \operatorname{holim} \prod_{[\sigma] \in \mathcal{B}_{\bullet} / G} \operatorname{Map}_{G}\left(G / K(\sigma), V_{G U}\right) \\
& \cong \operatorname{holim} \prod_{[\sigma] \in \mathcal{B}_{\bullet} / G} V_{G U}^{K(\sigma)}
\end{aligned}
$$

By Lemma 10.6.5, there are equivalences

$$
V_{G U}^{K(\sigma)} \simeq T A F(K(\sigma))
$$

REMARK 13.2.10. The coface maps of the semi-cosimplicial spectrum $Q^{\bullet}$ are all instances of the $E_{\infty}$ operations arising from Proposition 11.1.1. Thus, the spectrum $Q^{\bullet}$ is actually a semi-cosimplicial $E_{\infty}$-ring spectrum, and the totalization $Q$ therefore inherits the structure of an $E_{\infty}$-ring spectrum. 



\section{CHAPTER 14}

\section{$K(n)$-local theory}

Fix a compact open subgroup $K^{p}$ of $G U\left(\mathbb{A}^{p, \infty}\right)$ so that $S h\left(K^{p}\right)$ is a scheme. Let

$$
\mathbf{A}_{\text {univ }}=\left(A_{\text {univ }}, i_{\text {univ }}, \lambda_{\text {univ }},\left[\eta_{\text {univ }}\right]\right)
$$

be the universal tuple over $S h\left(K^{p}\right)$. Let $S h\left(K^{p}\right)^{[n]}$ be the reduced closed subscheme of $S h\left(K^{p}\right) \otimes_{\mathbb{Z}_{p}} \mathbb{F}_{p}$ where the the formal group $\epsilon A_{\text {univ }}(u)^{0}$ has height $n$ (see [HT01, Lem. II.1.1]).

\subsection{Endomorphisms of $\bmod p$ points}

Suppose that $\mathbf{A}=(A, i, \lambda,[\eta])$ is an element of $\operatorname{Sh}\left(K^{p}\right)^{[n]}\left(\overline{\mathbb{F}}_{p}\right)$.

We make the following definitions.

$$
\begin{aligned}
D & =\operatorname{End}_{B}^{0}(A), \\
\mathcal{O}_{D} & =\operatorname{End}_{B}(A), \\
\dagger & =\lambda \text {-Rosati involution on } D .
\end{aligned}
$$

By Theorem 2.2.4, $D$ is a central simple algebra over $F$ of dimension $n^{2}$, with invariants given by:

$$
\begin{aligned}
& \operatorname{inv}_{x} D=-\operatorname{inv}_{x} B, \quad x \not p, \\
& \operatorname{inv}_{u} D=1 / n, \\
& \operatorname{inv}_{\bar{u}} D=(n-1) / n .
\end{aligned}
$$

The ring $\mathcal{O}_{D}$ is an order in $D$. Since the $p$-divisible $\mathcal{O}_{B}$-module of $A$ takes the form

$$
A(p) \cong(\epsilon A(u))^{n} \times\left(\epsilon A\left(u^{c}\right)^{n}\right),
$$

Theorems 1.2.1(4) and 2.2.1 combine to show that the order $\mathcal{O}_{D,(p)}$ is maximal at $p$. Because the polarization $\lambda$ is prime-to- $p$, the order $\mathcal{O}_{D,(p)}$ is preserved by the Rosati involution $\dagger$.

Define algebraic groups $G U_{\mathbf{A}}$ and $U_{\mathbf{A}}$ over $\mathbb{Z}_{(p)}$ by

$$
\begin{aligned}
G U_{\mathbf{A}}(R) & =\left\{g \in\left(\mathcal{O}_{D,(p)} \otimes_{\mathbb{Z}_{(p)}} R\right)^{\times}: g^{\dagger} g \in R^{\times}\right\}, \\
U_{\mathbf{A}}(R) & =\left\{g \in\left(\mathcal{O}_{D,(p)} \otimes_{\mathbb{Z}_{(p)}} R\right)^{\times}: g^{\dagger} g=1\right\} .
\end{aligned}
$$

There is a short exact sequence

$$
1 \rightarrow U_{\mathbf{A}} \rightarrow G U_{\mathbf{A}} \stackrel{\nu}{\rightarrow} \mathbb{G}_{m} \rightarrow 1 .
$$


Observe that we have (Lemma 4.5.1)

$$
\begin{aligned}
G U_{\mathbf{A}}\left(\mathbb{Z}_{(p)}\right) & =\text { group of prime-to- } p \text { quasi-similitudes of }(A, i, \lambda), \\
U_{\mathbf{A}}\left(\mathbb{Z}_{(p)}\right) & =\text { group of prime-to- } p \text { quasi-isometries of }(A, i, \lambda) .
\end{aligned}
$$

The rational uniformization induces isomorphisms

$$
\begin{array}{r}
\eta_{*}: G U\left(\mathbb{Q}_{\ell}\right) \stackrel{\cong}{\cong} G U_{\mathbf{A}}\left(\mathbb{Q}_{\ell}\right), \\
\eta_{*}: U\left(\mathbb{Q}_{\ell}\right) \stackrel{\cong}{\cong} U_{\mathbf{A}}\left(\mathbb{Q}_{\ell}\right),
\end{array}
$$

for primes $\ell \neq p$. The maximality of the $\mathcal{O}_{F,(p)}$-order $\mathcal{O}_{D,(p)}$, together with Lemma 5.2.1, gives the following lemma.

LEMma 14.1.1. The induced action of $U_{\mathbf{A}}\left(\mathbb{Z}_{(p)}\right)$ on the summand $\epsilon A(u)$ of the $p$ divisible group $A(p)$ induces an isomorphism

$$
U_{\mathbf{A}}\left(\mathbb{Z}_{p}\right) \stackrel{\cong}{\longrightarrow} \mathbb{S}_{n}
$$

where $\mathbb{S}_{n}=\operatorname{Aut}(\epsilon A(u))$ is the nth Morava stabilizer group. The similitude norm gives a split short exact sequence

$$
1 \rightarrow U_{\mathbf{A}}\left(\mathbb{Z}_{p}\right) \rightarrow G U_{\mathbf{A}}\left(\mathbb{Z}_{p}\right) \stackrel{\nu}{\rightarrow} \mathbb{Z}_{p}^{\times} \rightarrow 1 .
$$

Define $\Gamma$ to be the quasi-isometry group $U_{\mathbf{A}}\left(\mathbb{Z}_{(p)}\right)$. The action of $\Gamma$ on the Tate module $V^{p, S}(A)$ induces an inclusion

$$
i_{\eta}: \Gamma \hookrightarrow U\left(\mathbb{A}^{p, S, \infty}\right) \subset G U\left(\mathbb{A}^{p, \infty}\right) .
$$

For any subgroup $K$ of $G U\left(\mathbb{A}^{p, S, \infty}\right)$ let $\Gamma(K)$ be the subgroup of $\Gamma$ given by the intersection $\Gamma \cap K$.

Proposition 14.1.2. Suppose that $K^{p}$ is an arbitrary open compact subgroup of $G U\left(\mathbb{A}^{p, \infty}\right)$, so that $S h\left(K^{p}\right)$ is not necessarily a scheme. Then the automorphisms of the $\overline{\mathbb{F}}_{p}$-point $\mathbf{A}=\left(A, i, \lambda,[\eta]_{K^{p}}\right)$ are given by $\Gamma\left(K^{p}\right)$.

Proof. By definition, we have

$$
\operatorname{Aut}(\mathbf{A})=G U_{\mathbf{A}}\left(\mathbb{Z}_{(p)}\right) \cap K^{p} \subset G U\left(\mathbb{A}^{p, \infty}\right)
$$

(using the isomorphism $\left.\eta_{*}: G U\left(\mathbb{A}^{p, \infty}\right) \stackrel{\cong}{\longrightarrow} G U_{\mathbf{A}}\left(\mathbb{A}^{p, \infty}\right)\right)$. The similitude norm restricts to give a homomorphism

$$
\nu: \operatorname{Aut}(\mathbf{A}) \rightarrow\{ \pm 1\}=\left(\widehat{\mathbb{Z}}^{p}\right)^{\times} \cap \mathbb{Z}_{(p)}^{\times} \subset\left(\mathbb{A}^{p, \infty}\right)^{\times} .
$$

However, by the positivity of the Rosati involution (Theorem 4.2.1), the similitude norm $\nu$ cannot be negative. We therefore deduce

$$
\operatorname{Aut}(\mathbf{A})=U_{\mathbf{A}}\left(\mathbb{Z}_{(p)}\right) \cap K^{p}=\Gamma\left(K^{p}\right) .
$$




\subsection{Approximation results}

In this section we compile various approximation results that we shall appeal to in later sections. These results both will allow us to compare the spectrum $Q_{U}\left(K^{p, S}\right)_{K(n)}$ with the $K(n)$-local sphere, as well as to manipulate certain adelic quotients.

For each prime $x \neq p$, let $K_{1, x}<G U\left(\mathbb{Q}_{x}\right)$ be the image of the subgroup

$$
\left\{g \in \mathcal{O}_{D, x}^{\times}: g^{\dagger} g \in \mathbb{Z}_{\ell}^{\times}\right\}<G U_{\mathbf{A}}\left(\mathbb{Q}_{\ell}\right)
$$

under the isomorphism $\eta_{*}^{-1}: G U_{\mathbf{A}}\left(\mathbb{Q}_{x}\right) \cong G U\left(\mathbb{Q}_{x}\right)$. For a set of primes $S$ not containing $p$, define the group $K_{1}^{p, S}$ by

$$
K_{1}^{p, S}=\prod_{x \notin\{p\} \cup S} K_{1, x}
$$

Naumann [Nau, Cor. 21, Rmk. 22] proves the following theorem, quantifying, at least in certain situations, the degree to which the group $\Gamma\left(K_{1}^{p, S}\right)$ approximates the Morava stabilizer group $\mathbb{S}_{n}$.

Theorem 14.2.1 (Naumann). Suppose that

(1) The polarization $\lambda$ is principal,

(2) The order $\mathcal{O}_{D}$ is maximal.

Then we have the following.

Case $\mathbf{n}$ odd: There exists a prime $\ell \neq p$ which splits in $F$ so that the group $\Gamma\left(K_{1}^{p, \ell}\right)$ is dense in $\mathbb{S}_{n}$.

Case $\mathbf{n}$ even: Suppose that $p \neq 2$. Then there exists a prime $\ell$ which splits in $F$ such that the closure of the group $\Gamma\left(K_{1}^{p, 2, \ell}\right)$ in $\mathbb{S}_{n}$ is of index less than or equal to the order of the unit group $\mathcal{O}_{F}^{\times}$.

REMARK 14.2.2. Since $F$ is a quadratic imaginary extension, $\mathcal{O}_{F}^{\times}$is of order 2,4 , or 6 .

A key observation of Naumann is the following proposition.

Proposition 14.2.3. Suppose that we have a map of short exact sequences groups

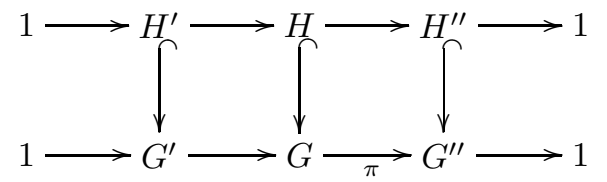

where the bottom row is a short exact sequence of first countable topological groups. Assume that $H^{\prime}$ is dense in $G^{\prime}$ and that there exists an open subgroup $U$ of $G^{\prime \prime}$ so that $\pi^{-1}(U)$ is compact in $G$. Then $H$ is dense in $\pi^{-1}\left(\bar{H}^{\prime \prime}\right)$, where $\bar{H}^{\prime \prime}$ is the closure of $H^{\prime \prime}$ in $G^{\prime \prime}$.

Naumann's methods may be used to prove the following easier proposition. 
Proposition 14.2.4.

(1) The group $U_{\mathbf{A}}(\mathbb{Q})$ is dense in $U_{\mathbf{A}}\left(\mathbb{Q}_{p}\right)$.

(2) The group $\Gamma=U_{\mathbf{A}}\left(\mathbb{Z}_{(p)}\right)$ is dense in $\mathbb{S}_{n}=U_{\mathbf{A}}\left(\mathbb{Z}_{p}\right)$.

(3) The group $G U_{\mathbf{A}}(\mathbb{Q})$ is dense in $G U_{\mathbf{A}}\left(\mathbb{Q}_{p}\right)$.

Proof. We prove statements (1) and (2) simultaneously. Let $S U_{\mathbf{A}}$ be the kernel of the reduced norm:

$$
1 \rightarrow S U_{\mathbf{A}} \rightarrow U_{\mathbf{A}} \stackrel{N_{D / F}}{\longrightarrow} T \rightarrow 1
$$

where the algebraic group $T / \mathbb{Z}_{(p)}$ is given by

$$
T(R)=\left\{t \in\left(\mathcal{O}_{F,(p)} \otimes_{\mathbb{Z}_{(p)}} R\right)^{\times}: N_{F / \mathbb{Q}}(t)=1\right\} .
$$

Using the fact that there is a pullback [PR94, 1.4.2]

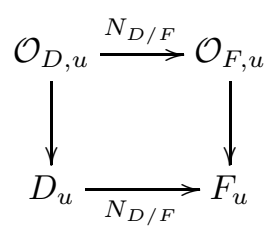

we deduce that the following diagram is a pullback.

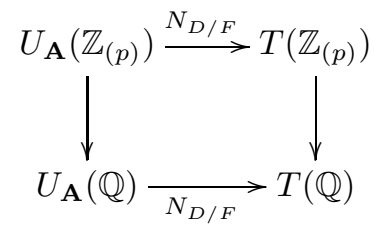

The weak approximation theorem [PR94, Lemma 7.2] implies that the embedding

$$
S U_{\mathbf{A}}(\mathbb{Q}) \hookrightarrow S U_{\mathbf{A}}\left(\mathbb{Q}_{p}\right)
$$

is dense. However, the pullback in diagram (14.2.5) implies that $S U_{\mathbf{A}}\left(\mathbb{Z}_{p}\right)=$ $S U_{\mathbf{A}}\left(\mathbb{Q}_{p}\right)$ and the pullback in diagram $(14.2 .6)$ implies that $S U_{\mathbf{A}}\left(\mathbb{Z}_{(p)}\right)=S U_{\mathbf{A}}(\mathbb{Q})$. So we actually have determined that the embedding

$$
S U_{\mathbf{A}}\left(\mathbb{Z}_{(p)}\right) \hookrightarrow S U_{\mathbf{A}}\left(\mathbb{Z}_{p}\right)
$$

is dense. In [Nau], it is established that the following maps are surjections:

$$
\begin{aligned}
& N_{D / F}: U_{\mathbf{A}}(\mathbb{Q}) \rightarrow T(\mathbb{Q}), \\
& N_{D / F}: U_{\mathbf{A}}\left(\mathbb{Z}_{p}\right) \rightarrow T\left(\mathbb{Z}_{p}\right) .
\end{aligned}
$$

The pullback of Diagram (14.2.6) implies that the map

$$
N_{D / F}: U_{\mathbf{A}}\left(\mathbb{Z}_{(p)}\right) \rightarrow T\left(\mathbb{Z}_{(p)}\right)
$$


is a surjection. We therefore have the following diagrams of short exact sequences.

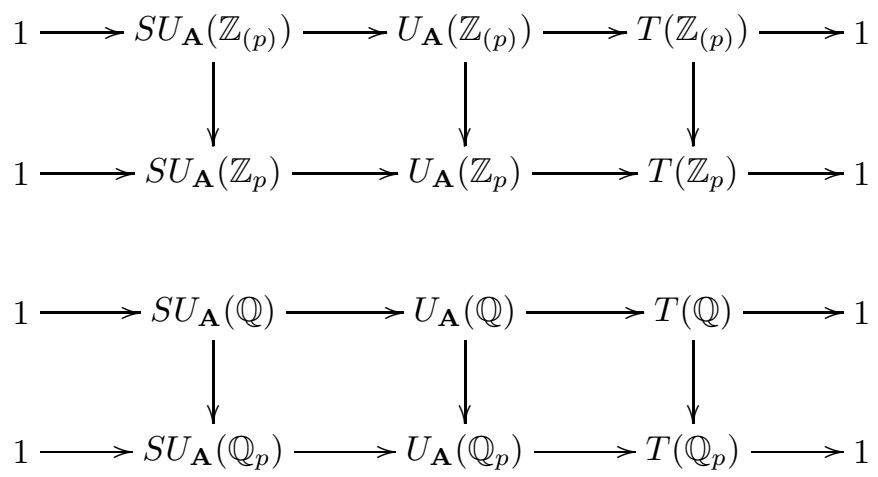

The groups $T\left(\mathbb{Z}_{(p)}\right)$ and $T(\mathbb{Q})$ are dense in $T\left(\mathbb{Z}_{p}\right)$ and $T\left(\mathbb{Q}_{p}\right)$, respectively (see, for instance, $[\mathbf{N a u}]$ ). Therefore, (1) and (2) follow from Proposition 14.2.3. (To verify that the second diagram above satisfies the hypotheses of Proposition 14.2.3 we need to again appeal to the pullback (14.2.5).)

By (1) and Proposition 14.2.3, to prove (3) it suffices to prove that the image of the similitude norm

$$
\nu: G U_{\mathbf{A}}(\mathbb{Q}) \rightarrow \mathbb{Q}^{\times}
$$

is dense in $\mathbb{Q}_{p}^{\times}$. Because the similitude norm restricts to the norm $N_{F / \mathbb{Q}}$ on the subgroup $F^{\times} \leq G U(\mathbb{Q})$, it suffices to prove that the image of the norm

$$
N_{F / \mathbb{Q}}: F^{\times} \rightarrow \mathbb{Q}^{\times}
$$

is dense in $\mathbb{Q}_{p}^{\times}$. Let $D$ be the absolute value of the discriminant of $F$, and let

$$
\chi:(\mathbb{Z} / D)^{\times} \rightarrow\{ \pm 1\}
$$

be the corresponding Dirichlet character, so that a prime $q$ splits in $F$ if and only if $\chi(q)=1$.

Assume that $p$ is odd. Fix a prime $\ell$ which splits in $F$ and is a generator of $\mathbb{Z}_{p}^{\times}$. Such a prime exists because the former represents a congruence condition modulo $D$, whereas the latter represents a congruence condition modulo $p^{2}$, and since $p$ was assumed to split, it is coprime to $D$. To prove the image of $N_{F / \mathbb{Q}}$ is dense in $\mathbb{Q}_{p}^{\times}$, it suffices to prove that $\ell$ and $p$ are in the image. By the fundamental short exact sequence

$$
1 \rightarrow \mathbb{Q}^{\times} / N\left(F^{\times}\right) \rightarrow \bigoplus_{x} \mathbb{Q}_{x}^{\times} / N\left(F_{x}^{\times}\right) \rightarrow \mathbb{Z} / 2 \rightarrow 1
$$

it suffices to prove that for all non-split $q$, both $p$ and $\ell$ are zero in the group $\mathbb{Q}_{q}^{\times} / N\left(F_{q}^{\times}\right)$. If $q$ is inert in $F$, then this follows from the fact that $p$ and $\ell$ are coprime to $q$. If $q$ is ramified, this follows from the fact that the kernel of the composite

$$
\mathbb{Z}_{q}^{\times} \rightarrow(\mathbb{Z} / D)^{\times} \stackrel{\chi}{\rightarrow}\{ \pm 1\}
$$

is equal to $N\left(F_{q}^{\times}\right) \cap \mathbb{Z}_{q}^{\times}$. The case of $p=2$ is similar, but because $\mathbb{Z}_{2}^{\times}$is not cyclic, two generating split primes must be used instead. 
Lemma 14.2.7. The images of the composites

$$
\begin{gathered}
G U_{\mathbf{A}}(\mathbb{Q}) \stackrel{\nu}{\rightarrow} \mathbb{Q}^{\times} \stackrel{\oplus \nu_{\ell}}{\longrightarrow} \bigoplus_{\ell \text { prime }} \mathbb{Z} \\
G U_{\mathbf{A}}\left(\mathbb{A}^{\infty}\right) \stackrel{\nu}{\rightarrow}\left(\mathbb{A}^{\infty}\right)^{\times} \stackrel{\oplus \nu_{\ell}}{\longrightarrow} \bigoplus_{\ell \text { prime }} \mathbb{Z}
\end{gathered}
$$

are equal.

Proof. The result follows from applying Galois cohomology computations of Section 5.3 to the map of exact sequences.

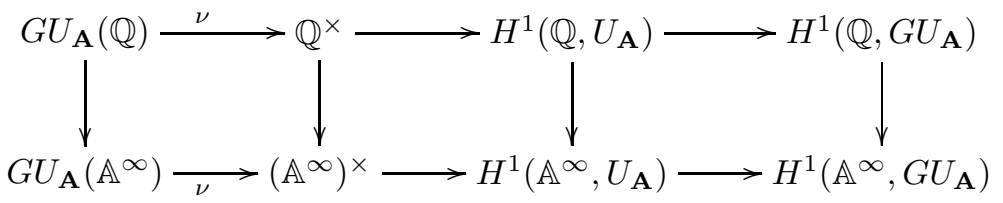

If $n$ is even, then the image of $\nu: G U(\mathbb{Q}) \rightarrow \mathbb{Q}^{\times}$is seen to be $\left(\mathbb{Q}^{\times}\right)^{+}$, and the theorem is clear. If $n$ is odd, then we have the following map of exact sequences.

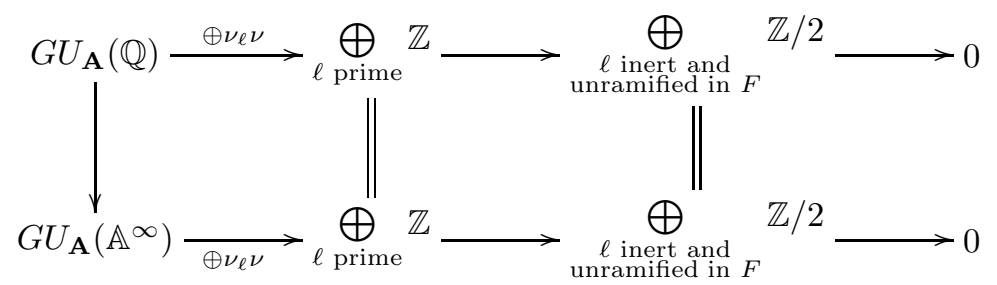

\subsection{The height $n$ locus of $S h\left(K^{p}\right)$}

Let $K^{p}$ be sufficiently small so that $S h\left(K^{p}\right)$ is a scheme. Combining Corollary 7.3.2 with Corollary II.1.4 of [HT01], we see that the subscheme $S h\left(K^{p}\right)^{[n]}$ is either empty, or smooth of dimension zero. We therefore have the following lemma.

Lemma 14.3.1. The scheme $\operatorname{Sh}\left(K^{p}\right)^{[n]}$ is étale over $\operatorname{Spec}\left(\mathbb{F}_{p}\right)$.

The scheme $S h\left(K^{p}\right)^{[n]}$ is therefore affine. Its structure is completely determined by

(1) the set of $\overline{\mathbb{F}}_{p}$-points $S h\left(K^{p}\right)^{[n]}\left(\overline{\mathbb{F}}_{p}\right)$,

(2) the action of the Galois group Gal of $\mathbb{F}_{p}$ on

$$
\operatorname{Sh}\left(K^{p}\right)^{[n]} \otimes_{\mathbb{F}_{p}} \overline{\mathbb{F}}_{p}=\coprod_{S h\left(K^{p}\right)^{[n]}\left(\overline{\mathbb{F}}_{p}\right)} \operatorname{Spec}\left(\overline{\mathbb{F}}_{p}\right) .
$$

Item (1) above is described by Corollary 14.3.4. Item (2) is a serious arithmetic question related to the zeta function of the Shimura variety - we do not investigate it here. 
Existence of $\bmod p$ points. The following proposition appears in [HT01, Cor V.4.5] in the case where $B$ is a division algebra.

Proposition 14.3.2. The set $\operatorname{Sh}\left(K^{p}\right)^{[n]}\left(\overline{\mathbb{F}}_{p}\right)$ is non-empty.

Proof. By Theorem 2.2.4, there exists a $B$-linear abelian variety $(A, i)$ over $\overline{\mathbb{F}}_{p}$ of dimension $n^{2}$ associated to the minimal $p$-adic type $(F, \zeta)$ where

$$
\begin{aligned}
\zeta_{u} & =1 / n, \\
\zeta_{u^{c}} & =(n-1) / n .
\end{aligned}
$$

The $p$-adic type of $(A, i)$ determines the slopes of the $p$-divisible group $A(p)$, and there is therefore an isogeny of $B$-linear $p$-divisible groups

$$
\phi:\left(A(p), i_{*}\right) \rightarrow\left(\mathbb{G}, i^{\prime}\right)
$$

such that

$$
\mathbb{G}=(\epsilon \mathbb{G}(u))^{n} \oplus\left(\epsilon \mathbb{G}\left(u^{c}\right)\right)^{n} .
$$

Since the $p$-completion of the order $\mathcal{O}_{B}$ is given by

$$
\mathcal{O}_{B, p}=M_{n}\left(\mathcal{O}_{F, u}\right) \times M_{n}\left(\mathcal{O}_{F, u^{c}}\right),
$$

the inclusion of rings

$$
i^{\prime}: B \hookrightarrow \operatorname{End}^{0}(\mathbb{G})
$$

lifts to an inclusion

$$
i^{\prime}: \mathcal{O}_{B,(p)} \hookrightarrow \operatorname{End}(\mathbb{G}) .
$$

Since $\phi$ is an isogeny, there exists a $k$ such that $\operatorname{ker} \phi$ is contained in the finite group-scheme $A\left[p^{k}\right]$ of $p^{k}$-torsion points of $A$. We define an isogenous $B$-linear abelian variety $\left(A^{\prime}, i^{\prime}\right)$ by taking the quotient

$$
(A, i) \rightarrow\left(A / \operatorname{ker} \phi, i^{\prime}\right)=\left(A^{\prime}, i^{\prime}\right) .
$$

There is a canonical isomorphism of $B$-linear $p$-divisible groups

$$
\left(A^{\prime}(p),\left(i^{\prime}\right)_{*}\right) \cong\left(\mathbb{G}, i^{\prime}\right) .
$$

By Theorem 2.2.1, the inclusion of rings

$$
i^{\prime}: B \hookrightarrow \operatorname{End}^{0}\left(A^{\prime}\right)
$$

lifts to an inclusion

$$
i^{\prime}: \mathcal{O}_{B,(p)} \hookrightarrow \operatorname{End}\left(A^{\prime}\right)_{(p)} .
$$

Choose a compatible polarization

$$
\lambda: A^{\prime} \rightarrow\left(A^{\prime}\right)^{\vee}
$$

and let $\lambda_{*}: A(p) \rightarrow A(p)^{\vee}$ be the induced map of $p$-divisible groups. (According to Lemma 4.6.1, compatible polarizations exist.) Since $\lambda$ is compatible, there exist finite subgroups $K_{u}<A(u)$ and $K_{u^{c}}<A(\bar{u})$ such that

$$
\operatorname{ker} \lambda_{*}=K_{u} \oplus K_{u^{c}}<A(u) \oplus A\left(u^{c}\right)=A(p) .
$$

Define $A^{\prime \prime}$ to be the quotient $A^{\prime} / K_{u}$, with quotient isogeny $q: A^{\prime} \rightarrow A^{\prime \prime}$. Compatibility of the polarization implies that the $p$-divisible group $A^{\prime}(u) / K_{u} \oplus A(\bar{u})$ inherits a $\mathcal{O}_{B,(p)}$-linear structure, giving an inclusion

$$
i^{\prime \prime}: \mathcal{O}_{B,(p)} \hookrightarrow \operatorname{End}\left(A^{\prime \prime}\right) .
$$


The quasi-isogeny

$$
\lambda^{\prime}=\left(q^{-1}\right)^{\vee} \lambda q^{-1}: A^{\prime \prime} \rightarrow\left(A^{\prime \prime}\right)^{\vee}
$$

is easily seen to be prime-to- $p$, and thus gives a prime-to- $p$ compatible polarization on $\left(A^{\prime \prime}, i^{\prime \prime}\right)$.

In order to produce a point of $S h\left(K^{p}\right)^{[n]}\left(\overline{\mathbb{F}}_{p}\right)$, we need to show that the polarization $\lambda^{\prime}$ can be altered so that there exists a similitude between the Weil pairing on the Tate module $V^{p}\left(A^{\prime \prime}\right)$ and our fixed pairing $\langle-,-\rangle$ on $V^{p}$. If $n$ is odd, then for all $\ell, H^{1}\left(\mathbb{Q}_{\ell}, G U\right)=0$ (Lemma 5.3.1 and Corollary 5.3.4), and so any two nondegenerate $*$-hermitian alternating forms on $V_{\ell} \cong V_{\ell}\left(A^{\prime \prime}\right)$ are similar, so there exists a uniformization

$$
\eta:\left(V^{p},\langle-,-\rangle\right) \stackrel{\simeq}{\longrightarrow}\left(V^{p}\left(A^{\prime \prime}\right), \lambda^{\prime}\langle-,-\rangle\right)
$$

We must work harder if $n$ is even. Using Theorem 7.3.1, there exists a deformation $\left(\widetilde{A}^{\prime \prime}, \widetilde{i}^{\prime \prime}, \widetilde{\lambda}^{\prime}\right)$ of the tuple $\left(A^{\prime \prime}, i^{\prime \prime}, \lambda^{\prime}\right)$ over the Witt ring $W\left(\overline{\mathbb{F}}_{p}\right)$. Choose an embedding of the field $F_{u}^{n r} \cong W\left(\overline{\mathbb{F}}_{p}\right) \otimes \mathbb{Q}$ in $\mathbb{C}$ so that the following diagram of fields commutes.

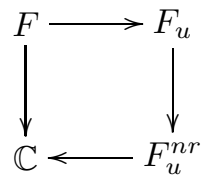

Under this inclusion, we may pull back $\left(\widetilde{A}^{\prime \prime}, \tilde{i^{\prime \prime}}, \tilde{\lambda}\right)$ to a polarized $\mathcal{O}_{B,(p)}$-linear abelian variety $\left(A_{\mathbb{C}}^{\prime \prime}, i_{\mathbb{C}}^{\prime \prime}, \lambda_{\mathbb{C}}^{\prime}\right)$ over $\mathbb{C}$. The methods of Section 9.3 imply that there is a non-degenerate $*$-hermitian alternating form $\langle-,-\rangle^{\prime}$ on $V$, a lattice $L^{\prime} \subset V$, and a compatible complex structure $J$ on $V_{\infty}=V \otimes_{\mathbb{Q}} \mathbb{R}$ so that:

$$
\begin{aligned}
A_{\mathbb{C}}^{\prime \prime} & =V_{\infty} / L^{\prime}, \\
i_{\mathbb{C}}^{\prime \prime} & =\text { induced from } B \text {-module structure of } V, \\
\lambda_{\mathbb{C}}^{\prime \prime} & =\text { polarization associated to Riemann form }\langle-,-\rangle^{\prime} .
\end{aligned}
$$

Thus, there is a canonical uniformization

$$
\eta_{1}:\left(V^{p},\langle-,-\rangle^{\prime}\right) \stackrel{\simeq}{\longrightarrow}\left(V^{p}\left(A_{\mathbb{C}}^{\prime \prime}\right), \lambda_{\mathbb{C}}^{\prime}\langle-,-\rangle\right) .
$$

Furthermore, there are isomorphisms (using proper-smooth base change):

$$
\begin{aligned}
\left(V^{p}\left(A_{\mathbb{C}}^{\prime \prime}\right), \lambda_{\mathbb{C}}^{\prime}\langle-,-\rangle\right) & \cong\left(H_{e t}^{1}\left(A_{\mathbb{C}}^{\prime \prime}, \mathbb{A}^{p, \infty}\right)^{*}, \lambda_{\mathbb{C}}^{\prime}\langle-,-\rangle\right) \\
& \cong\left(H_{e t}^{1}\left(\widetilde{A}^{\prime \prime}, \mathbb{A}^{p, \infty}\right)^{*}, \widetilde{\lambda}^{\prime}\langle-,-\rangle\right) \\
& \cong\left(H_{e t}^{1}\left(A^{\prime \prime}, \mathbb{A}^{p, \infty}\right)^{*}, \lambda^{\prime}\langle-,-\rangle\right) \\
& \cong\left(V^{p}\left(A^{\prime \prime}\right), \lambda^{\prime}\langle-,-\rangle\right) .
\end{aligned}
$$

Let $G U_{\mathbf{A}^{\prime \prime}}$ be the group of quasi-similitudes of $\mathbf{A}^{\prime \prime}=\left(A^{\prime \prime}, i^{\prime \prime}, \lambda^{\prime}\right)$. By Lemma 4.6.1, any other isogeny class of weak polarization $\lambda^{\prime \prime}$ is determined by an element $\left[\lambda^{\prime \prime}\right] \in$ $H^{1}\left(\mathbb{Q}, G U_{\mathbf{A}^{\prime \prime}}\right)$ such that

$$
\left[\lambda^{\prime}\right]_{\infty}=\left[\lambda^{\prime \prime}\right]_{\infty} \in H^{1}\left(\mathbb{R}, G U_{\mathbf{A}^{\prime \prime}}\right)
$$


The calculations of Section 5.3 give isomorphisms:

$$
\begin{aligned}
& \operatorname{disc}_{\dagger^{\prime}}: H^{1}\left(\mathbb{Q}_{\ell}, G U_{\mathbf{A}^{\prime \prime}}\right) \cong \\
& \operatorname{disc}_{*}: H^{1}\left(\mathbb{Q}_{\ell}, G U\right) \cong N_{F / \mathbb{Q}}\left(F^{\times}\right), \\
& \mathbb{Q}_{\ell}^{\times} / N_{F / \mathbb{Q}}\left(F^{\times}\right) .
\end{aligned}
$$

In particular, $H^{1}\left(\mathbb{Q}_{p}, G U\right)=H^{1}\left(\mathbb{Q}_{p}, G U_{\mathbf{A}^{\prime \prime}}\right)=0$. Here, disc †े' $_{\dagger^{\prime}}$ denotes the discriminant taken relative to the $\lambda^{\prime}$-Rosati involution $(-)^{\dagger^{\prime}}$, and disc ${ }_{*}$ denotes the discriminant taken relative to the involution $(-)^{*}$. We identify the Galois cohomologies $H^{1}\left(\mathbb{Q}_{\ell}, G U_{\mathbf{A}^{\prime \prime}}\right)$ and $H^{1}\left(\mathbb{Q}_{\ell}, G U\right)$ in this manner, and use these isomorphisms to endow these isomorphic sets with the structure of a group. By Lemma 4.6.3, the image of $\left[\lambda^{\prime \prime}\right]$ in $H^{1}\left(\mathbb{Q}_{\ell}, G U\right)$ is given by

$$
\left[\lambda^{\prime \prime}\right]_{\ell}=\left[\lambda^{\prime}\langle-,-\rangle_{\ell}\right]+\left[\lambda^{\prime \prime}\langle-,-\rangle_{\ell}\right],
$$

the difference of the classes represented by the Weil pairings on $V_{\ell}\left(A^{\prime \prime}\right)$. Let $\left[\langle-,-\rangle^{\prime}\right]$ be the element of $H^{1}(\mathbb{Q}, G U)$ representing the similitude class of the form $\langle-,-\rangle^{\prime}$. We wish to demonstrate that there is such a class $\left[\lambda^{\prime \prime}\right]$ so that

$$
\left[\lambda^{\prime \prime}\langle-,-\rangle_{\ell}\right]=[\langle-,-\rangle]_{\ell} \in H^{1}\left(\mathbb{Q}_{\ell}, G U\right)
$$

for every $\ell \neq p$. There are short exact sequences (Corollary 5.3.7)

$$
\begin{aligned}
0 & \rightarrow H^{1}(\mathbb{Q}, G U) \rightarrow \bigoplus_{x} H^{1}\left(\mathbb{Q}_{x}, G U\right) \stackrel{\sum \xi_{x}^{\prime}}{\longrightarrow} \mathbb{Z} / 2 \rightarrow 0, \\
0 & \rightarrow H^{1}\left(\mathbb{Q}, G U_{\mathbf{A}^{\prime \prime}}\right) \rightarrow \bigoplus_{x} H^{1}\left(\mathbb{Q}_{x}, G U_{\mathbf{A}^{\prime \prime}}\right) \stackrel{\sum \xi_{x}^{\prime}}{\longrightarrow} \mathbb{Z} / 2 \rightarrow 0 .
\end{aligned}
$$

By the positivity of the Rosati involution (Theorem 4.2.1), we have $\xi_{\infty}^{\prime}\left[\lambda^{\prime}\right]=0$. It therefore suffices to show that

$$
\sum_{\ell}\left(\xi_{\ell}^{\prime}\left([\langle-,-\rangle]_{\ell}\right)+\xi_{\ell}^{\prime}\left(\left[\lambda^{\prime}\langle-,-\rangle_{\ell}\right)\right]\right)=0 .
$$

Since the complex structure $J$ on $V_{\infty}$ given above is compatible with $\langle-,-\rangle^{\prime}$, the form $\langle-,-\rangle^{\prime}$ has signature $\{1, n-1\}$, and hence, by Lemma 5.3.2, we have

$$
[\langle-,-\rangle]_{\infty}=\left[\langle-,-\rangle^{\prime}\right]_{\infty} \in H^{1}(\mathbb{R}, G U)
$$

We compute

$$
\begin{aligned}
\sum_{\ell} \xi_{\ell}^{\prime}\left([\langle-,-\rangle]_{\ell}\right)+\sum_{\ell} \xi_{\ell}^{\prime}\left(\left[\lambda^{\prime}\langle-,-\rangle_{\ell}\right]\right) & =\sum_{\ell} \xi_{\ell}^{\prime}\left([\langle-,-\rangle]_{\ell}\right)+\sum_{\ell} \xi_{\ell}^{\prime}\left(\left[\langle-,-\rangle^{\prime}\right]_{\ell}\right) \\
& =\xi_{\infty}^{\prime}\left([\langle-,-\rangle]_{\infty}\right)+\xi_{\infty}^{\prime}\left(\left[\langle-,-\rangle^{\prime}\right]_{\infty}\right) \\
& =0 .
\end{aligned}
$$

Thus there exist a class $\left[\lambda^{\prime \prime}\right] \in H^{1}(\mathbb{Q}, G U)$ with $\left[\lambda^{\prime \prime}\langle-,-\rangle_{\ell}\right]=[\langle-,-\rangle]_{\ell}$ and $\left[\lambda^{\prime \prime}\right]_{\infty}=$ $\left[\lambda^{\prime}\right]_{\infty}$. There exists a corresponding polarization $\lambda^{\prime \prime}$. Using the same methods used to construct $\lambda^{\prime}$, we may assume without loss of generality that $\lambda^{\prime \prime}$ is prime-to- $p$.

Calculation of $S h\left(K^{p}\right)^{[n]}\left(\overline{\mathbb{F}}_{p}\right)$. We shall make use of the following lemma.

Proposition 14.3.3. Given any two tuples

$$
(A, i, \lambda,[\eta]),\left(A^{\prime}, i^{\prime}, \lambda^{\prime},\left[\eta^{\prime}\right]\right) \in \operatorname{Sh}\left(K^{p}\right)^{[n]}\left(\overline{\mathbb{F}}_{p}\right)
$$

there exists a prime-to-p isogeny

$$
(A, i, \lambda) \rightarrow\left(A^{\prime}, i^{\prime}, \lambda^{\prime}\right)
$$


of weakly polarized B-linear abelian varieties.

Proof. The completion $\mathcal{O}_{D, u}$ is the unique maximal order of the central $\mathbb{Q}_{p^{-}}$ division algebra $D_{u}$ of Hasse invariant $1 / n$. Let $S$ be a uniformizer of the maximal ideal $\mathfrak{m}_{D, u}$ of $\mathcal{O}_{D, u}$. There is an isomorphism $U_{\mathbf{A}}\left(\mathbb{Q}_{p}\right) \cong D_{u}^{\times}$(Lemma 14.1.1). By Proposition 14.2.4(1), there exists an element $T \in U_{\mathbf{A}}(\mathbb{Q})$ such that the image $T_{u} \in D_{u}^{\times}$satisfies

$$
T_{u}=S(1+S x)
$$

for some element $x \in D_{u}$. In particular, $T$ is a $B$-linear quasi-isometry

$$
T:(A, i, \lambda) \rightarrow(A, i, \lambda)
$$

for whose norm has $u$-valuation $\nu_{u}\left(N_{D / F}(T)\right)=1$. Lemma 14.2.7 implies that there exists an element $R \in G U_{\mathbf{A}}(\mathbb{Q})$ such that $p$-adic valuation of the similitude norm satisfies $\nu_{p}(\nu(R))=1$.

By Theorem 2.2.3 and Lemma 4.6.1, there exists a $B$-linear quasi-similitude

$$
\alpha:(A, i, \lambda) \rightarrow\left(A^{\prime}, i^{\prime}, \lambda^{\prime}\right) .
$$

There exists an integer $e_{1}$ so that

$$
\left(\alpha R^{e_{1}}\right)^{*} \lambda^{\prime}=c \lambda
$$

for some $c \in \mathbb{Z}_{(p)}^{\times}$. By altering $\lambda^{\prime}$ within its $\mathbb{Z}_{(p)}^{\times}$-weak polarization class, we may assume that $c=1$. Then $\alpha R^{e_{1}}$ is an quasi-isometry between $B$-linear abelian varieties with prime-to- $p$ polarizations. There exists an integer $e_{2}$ so that induced quasi-isogeny

$$
\left(\alpha R^{e_{1}}\right)_{*} S^{e_{2}}: \epsilon A(u) \rightarrow \epsilon A^{\prime}(u)
$$

is an isomorphism of formal groups. By Theorem 2.2.1 the following diagram is a pullback.

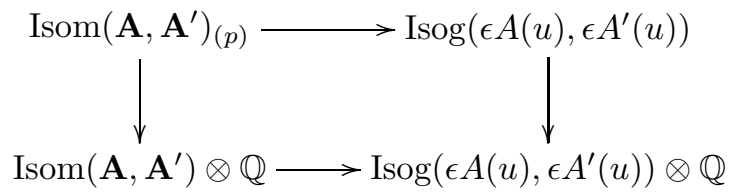

Here, $\operatorname{Isom}(-,-)$ denotes the isometries between $B$-linear polarized abelian varieties, and Isog denotes isogenies between $p$-typical groups. We deduce that

$$
\alpha R^{e_{1}} T^{e_{2}}:(A, i, \lambda) \rightarrow\left(A^{\prime}, i^{\prime}, \lambda^{\prime}\right)
$$

is a prime-to- $p$ isometry of $B$-linear polarized abelian varieties.

Corollary 14.3.4. The map

$$
G U_{\mathbf{A}}\left(\mathbb{Z}_{(p)}\right) \backslash G U\left(\mathbb{A}^{p, \infty}\right) / K^{p} \rightarrow \operatorname{Sh}\left(K^{p}\right)^{[n]}\left(\overline{\mathbb{F}}_{p}\right)
$$

given by sending a double coset $[g]$ to the tuple $\left(A, i, \lambda,[\eta g]_{K^{p}}\right)$ is an isomorphism.

We give an alternative characterizations of the adelic quotient of Corollary 14.3.4.

LEMMA 14.3.5. 
(1) The natural map

$$
\Gamma \backslash G U^{1}\left(\mathbb{A}^{p, \infty}\right) \rightarrow G U_{\mathbf{A}}\left(\mathbb{Z}_{(p)}\right) \backslash G U\left(\mathbb{A}^{p, \infty}\right)
$$

is an isomorphism.

(2) The natural map

$$
\Gamma \backslash G U^{1}\left(\mathbb{A}^{p, \infty}\right) / K^{p} \rightarrow G U_{\mathbf{A}}\left(\mathbb{Z}_{(p)}\right) \backslash G U\left(\mathbb{A}^{p, \infty}\right) / K^{p}
$$

is an isomorphism.

(3) The natural map

$G U_{\mathbf{A}}\left(\mathbb{Z}_{(p)}\right) \backslash G U\left(\mathbb{A}^{p, \infty}\right) / K^{p} \rightarrow G U_{\mathbf{A}}(\mathbb{Q}) \backslash G U_{\mathbf{A}}\left(\mathbb{A}^{\infty}\right) / K^{p} G U_{\mathbf{A}}\left(\mathbb{Z}_{p}\right)$

is an isomorphism.

Proof. (1) follows from Lemma 14.2.7, and the fact that $\Gamma=G U_{\mathbf{A}}\left(\mathbb{Z}_{(p)}\right) \cap$ $G U^{1}\left(\mathbb{A}^{p, \infty}\right)$ (Theorem 4.2.1), and (1) implies (2). Statement (3) follows from Proposition 14.2.4(3).

REMARK 14.3.6. Lemma 14.3.5 relates the number of $\overline{\mathbb{F}}_{p}$-points of $S h\left(K^{p}\right)^{[n]}$ to a class number of $G U_{\mathbf{A}}$.

\section{4. $K(n)$-local $T A F$}

In this section, fix an open compact subgroup $K^{p}$ of $G U\left(\mathbb{A}^{p, \infty}\right)$, sufficiently small so that $S h\left(K^{p}\right)$ is a scheme.

Lemma 14.4.1. Let $U \rightarrow S h\left(K^{p}\right)_{p}^{\wedge}$ be an étale open. Then the spectrum of sections $\mathcal{E}\left(K^{p}\right)(U)$ is $E(n)$-local. In particular, the spectrum $T A F\left(K^{p}\right)$ is $E(n)$-local.

Proof. Fix a formal affine étale open

$$
f: U^{\prime}=\operatorname{Spf}(R) \rightarrow U .
$$

Let $\left(A_{f}, i_{f}, \lambda_{f},\left[\eta_{f}\right]\right) / R$ be the tuple classified by $f$. The spectrum of sections $\mathcal{E}\left(K^{p}\right)\left(U^{\prime}\right)$ is Landweber exact (Corollary 8.1.7), therefore it is an $M U$-module spectrum ([HS99, Thm 2.8]). Greenlees and May [GM95, Thm 6.1] express the Bousfield localization at $E(n)$ as the localization with respect to the regular ideal $I_{n+1}=\left(p, v_{1}, \ldots, v_{n}\right)$ of $M U_{*}$ :

$$
\mathcal{E}\left(K^{p}\right)\left(U^{\prime}\right)_{E(n)} \simeq \mathcal{E}\left(K^{p}\right)\left(U^{\prime}\right)\left[I_{n+1}^{-1}\right] .
$$

There is a spectral sequence [GM95, Thm 5.1]:

$$
H^{s}\left(\operatorname{Spec}(R)-V, \omega^{\otimes t}\right) \Rightarrow \pi_{2 t-s} \mathcal{E}\left(K^{p}\right)\left(U^{\prime}\right)\left[I_{n+1}^{-1}\right] .
$$

Here $V$ is the locus of $\operatorname{Spec}(R / p)$ where the formal group $\epsilon A_{f}[u]^{0}$ is of height greater than $n$. However, the formal group cannot have height greater that the height of the height $n p$-divisible group $\epsilon A(u)$. Therefore $V$ is empty, and the spectral sequence collapses, because $\operatorname{Spec}(R)$ is affine. We conclude that the map

$$
\pi_{*} \mathcal{E}\left(K^{p}\right)\left(U^{\prime}\right) \rightarrow \pi_{*} \mathcal{E}\left(K^{p}\right)\left(U^{\prime}\right)_{E(n)}
$$

is an isomorphism, so $\mathcal{E}\left(K^{p}\right)\left(U^{\prime}\right)$ must be $E(n)$-local. 
Because $S h\left(K^{p}\right)$ is quasi-projective, $U$ is separated, hence all of the terms of the Čech nerve $\left(U^{\prime \bullet+1}\right)$

$$
U^{\prime \bullet+1}=\left\{U^{\prime} \Leftarrow U^{\prime} \times_{U} U^{\prime} \Leftarrow U^{\prime} \times_{U} U^{\prime} \times_{U} U^{\prime} \cdots\right\}
$$

are affine formal schemes. Because the sheaf $\mathcal{E}\left(K^{p}\right)$ satisfies homotopy descent, the map

$$
\mathcal{E}\left(K^{p}\right)(U) \rightarrow \underset{\Delta}{\operatorname{holim}} \mathcal{E}\left(K^{p}\right)\left(U^{\prime \bullet+1}\right)
$$

is an equivalence. Since the terms of the homotopy totalization are $E(n)$-local, we determine that $\mathcal{E}\left(K^{p}\right)(U)$ is $E(n)$-local.

Let Gal be the Galois group $\operatorname{Gal}\left(\overline{\mathbb{F}}_{p} / \mathbb{F}_{p}\right) \cong \widehat{\mathbb{Z}}$, generated by the Frobenius automorphism Fr. Let $S h\left(K^{p}\right)_{\mathbb{F}_{p^{k}}}$ be the Galois cover

$$
\operatorname{Sh}\left(K^{p}\right)_{\mathbb{F}_{p^{k}}}=S h\left(K^{p}\right) \otimes_{\mathbb{Z}_{p}} W\left(\mathbb{F}_{p^{k}}\right) \rightarrow \operatorname{Sh}\left(K^{p}\right) .
$$

Define spectra

$$
T A F\left(K^{p}\right)_{\mathbb{F}_{p^{k}}}=\mathcal{E}\left(K^{p}\right)\left(\left(S h\left(K^{p}\right)_{\mathbb{F}_{p^{k}}}\right)_{p}^{\wedge}\right) .
$$

The functoriality of the presheaf $\mathcal{E}\left(K^{p}\right)$ gives rise to a contravariant functor $\Phi$ on the subcategory $\mathrm{Orb}_{\text {Gal }}^{s m} \subset \mathrm{Set}_{\text {Gal }}^{s m}$ of smooth (i.e. finite) Gal-orbits by

$$
\Phi: G a l / H \mapsto T A F\left(K^{p}\right)_{\bar{F}_{p}^{H}} .
$$

Let

$$
T A F\left(K^{p}\right)_{\mathbb{F}_{p}}=V_{\Phi} \simeq \underset{k}{\lim _{k}} \operatorname{TAF}\left(K^{p}\right)_{\mathbb{F}_{p k}}
$$

be the associated associated smooth $\mathrm{Gal}$-spectrum of Construction 10.6.3.

Lemma 14.4.3. The spectrum TAF $\left(K^{p}\right)_{\mathbb{F}_{p}}$ is E(n)-local.

Proof. The underlying spectrum of $T A F\left(K^{p}\right)_{\mathbb{F}_{p}}$ is weakly equivalent to a colimit of $E(n)$-local spectra. Since localization with respect to $E(n)$ is smashing, the colimit is $E(n)$-local.

By Lemma 10.6.5, we can recover $T A F\left(K^{p}\right)$ by taking Gal-homotopy fixed points:

$$
T A F\left(K^{p}\right) \simeq\left(T A F\left(K^{p}\right)_{\overline{\mathbb{F}}_{p}}\right)^{h G a l} .
$$

By Theorem 5.2.5 of [Beh], we have the following lemma

LEMma 14.4.4. The sequence

$$
T A F\left(K^{p}\right) \rightarrow T A F\left(K^{p}\right)_{\mathbb{F}_{p}} \stackrel{\mathrm{Fr}-1}{\longrightarrow} T A F\left(K^{p}\right)_{\overline{\mathbb{F}}_{p}}
$$

is a fiber sequence.

For a multi-index $J=\left(j_{0}, j_{1}, \ldots, j_{n-1}\right)$, let $M(J)$ denote the corresponding generalized Moore spectrum with $B P$-homology

$$
B P_{*} M(J)=B P_{*} /\left(p^{j_{0}}, v_{1}^{j_{1}}, \ldots, v_{n-1}^{j_{n-1}}\right) .
$$

The periodicity theorem of Hopkins and Smith [HS98] guarantees the existence of generalized Moore spectra $M(J)$ for a cofinal collection of multi-indices $J$. The 
$K(n)$-localization of an $E(n)$-local spectrum $X$ may be calculated by by the following proposition ([HS99, Prop 7.10]).

Proposition 14.4.5. Suppose that $X$ is an $E(n)$-local spectrum. Then there is an equivalence

$$
X_{K(n)} \simeq \operatorname{holim}_{J} X \wedge M(J) .
$$

There is an isomorphism

$$
\operatorname{Sh}\left(K^{p}\right)_{\mathbb{F}_{p^{k}}}^{[n]} \cong \coprod_{i \in I\left(K^{p}, k\right)} \operatorname{Spec}\left(\mathbb{F}_{p^{k_{i}}}\right)
$$

for some finite index set $I\left(K^{p}, k\right)$. The numbers $k_{i}$ are all greater than or equal to $k$. Let $\overline{\mathbb{G}}_{i}$ be the restriction of the formal group $\epsilon A(u)^{0}$ to the $i$ th factor.

Proposition 14.4.6. There is an canonical equivalence

$$
\operatorname{TAF}\left(K^{p}\right)_{\mathbb{F}_{p^{k}}, K(n)} \stackrel{\simeq}{\longrightarrow} \prod_{i \in I\left(K^{p}, k\right)} E_{\overline{\mathbb{G}}_{i}} .
$$

Here $E_{\overline{\mathbb{G}}_{i}}$ is the Morava $E$-theory spectrum associated to the height $n$ formal group $\overline{\mathbb{G}}_{i}$ over $\mathbb{F}_{p^{k_{i}}}$.

Proof. Let $\left(S h\left(K^{p}\right)_{\mathbb{F}_{p^{k}}}\right)_{I_{n}}^{\wedge}$ denote the completion of $S h\left(K^{p}\right)_{\mathbb{F}_{p^{k}}}$ at the subscheme $S h\left(K^{p}\right)_{\mathbb{F}_{p^{k}}}^{[n]}$. By Corollary 7.3.2, there is an isomorphism

$$
\left(S h\left(K^{p}\right)_{\mathbb{F}_{p^{k}}}\right)_{I_{n}}^{\wedge} \cong \coprod_{i \in I\left(K^{p}, k\right)} \operatorname{Def}_{\overline{\mathbb{G}}_{i}}
$$

Let $g$ be the inclusion

$$
g:\left(S h\left(K^{p}\right)_{\mathbb{F}_{p^{k}}}\right)_{I_{n}}^{\wedge} \rightarrow\left(S h\left(K^{p}\right)_{\mathbb{F}_{p^{k}}}\right)_{p}^{\wedge}
$$

Let $f: U \rightarrow\left(S h\left(K^{p}\right)_{\mathbb{F}_{p^{k}}}\right)_{p}^{\wedge}$ be a formal affine étale cover, and let $U^{\bullet+1}$ be its Čech nerve. The cover $U$ pulls back to an étale cover $\widehat{f}: \widehat{U} \rightarrow\left(S h\left(K^{p}\right)_{\mathbb{F}_{p^{k}}}\right)_{I_{n}}$, with Čech nerve $\widehat{U}^{\bullet+1}$. There are isomorphisms

$$
\widehat{U}^{s} \cong \coprod_{i \in I\left(K^{p}, k, s\right)} \operatorname{Def}_{\overline{\mathbb{G}}_{s, i}}
$$

for some finite index set $I\left(K^{p}, k, s\right)$. The unit of the adjunction $\left(g^{*}, g_{*}\right)$ gives rise to a map of spectra of sections

$$
\operatorname{Res}_{g}: \mathcal{E}\left(K^{p}\right)\left(U^{s}\right) \rightarrow\left(g^{*} \mathcal{E}\left(K^{p}\right)\right)\left(\widehat{U}^{s}\right)
$$

The functoriality of Theorem 8.1.4, together with Corollary 8.1.9, gives a map

$$
(g, I d)^{*}:\left(g^{*} \mathcal{E}\left(K^{p}\right)\right)\left(\widehat{U}^{s}\right) \rightarrow \prod_{i \in I\left(K^{p}, k, s\right)} E_{\overline{\mathbb{G}}_{s, i}} .
$$

Since $U^{s}$ is a affine formal scheme, the homotopy groups of $\mathcal{E}\left(K^{p}\right)\left(U^{s}\right)$ is given by

$$
\pi_{k} \mathcal{E}\left(K^{p}\right)\left(U^{s}\right) \cong \begin{cases}\omega^{\otimes k / 2}\left(U^{s}\right) & k \text { even } \\ 0 & k \text { odd }\end{cases}
$$


We have the following diagram.

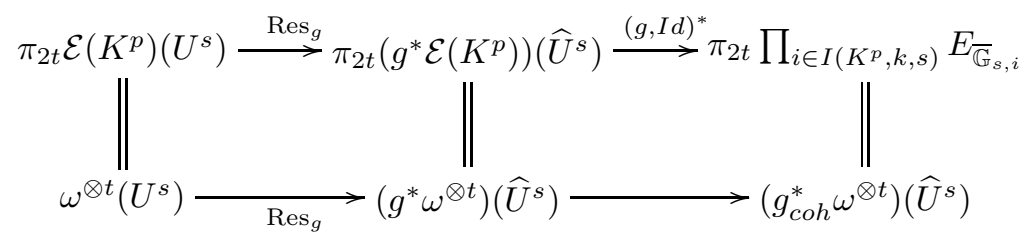

Here, $g^{*}$ denotes the pullback of sheaves of spectra/abelian groups whereas $g_{c o h}^{*}$ denotes the pullback of coherent sheaves. The bottom row induces an isomorphism

$$
\omega^{\otimes t}\left(U^{s}\right)_{I_{n}}^{\wedge} \cong\left(g_{c o h}^{*} \omega^{\otimes t}\right)\left(\widehat{U}^{s}\right),
$$

where we have completed the $M U_{*}$-module $\omega^{\otimes t}\left(U^{s}\right)$ at the ideal

$$
I_{n}=\left(p, v_{1}, \ldots, v_{n-1}\right) \subset M U_{*} .
$$

Therefore, we deduce that the map $\operatorname{Res}_{g} \circ(g, I d)^{*}$ induces an equivalence

$$
\operatorname{holim}_{J} \mathcal{E}\left(K^{p}\right)\left(U^{s}\right) \wedge M(J) \stackrel{\simeq}{\longrightarrow} \prod_{i \in I\left(K^{p}, k, s\right)} E_{\overline{\mathbb{G}}_{s, i}} .
$$

Taking holim$_{\Delta}$, we get an equivalence

$$
\operatorname{holim} \operatorname{holim}_{J} \mathcal{E}\left(K^{p}\right)\left(U^{\bullet+1}\right) \wedge M(J) \stackrel{\simeq}{\rightarrow} \operatorname{holim}_{\Delta} \prod_{i \in I\left(K^{p}, k, \bullet+1\right)} E_{\overline{\mathbb{G}}_{\bullet+1, i}} .
$$

(The cosimplicial structure of the target is induced by the simplicial structure of the Čech nerve $\widehat{U}^{\bullet+1}$ using the functoriality of Theorem 8.1.3.) Using Lemma 14.4.1, Proposition 14.4.5, the homotopy descent property for the presheaf $\mathcal{E}\left(K^{p}\right)$, and the fact that the complexes $M(J)$ are finite, we have equivalences

$$
\begin{aligned}
\underset{\Delta}{\operatorname{holim}} \operatorname{holim}_{J} \mathcal{E}\left(K^{p}\right)\left(U^{\bullet+1}\right) \wedge M(J) & \simeq \operatorname{holim}_{J} M(J) \wedge \operatorname{holim}_{\Delta} \mathcal{E}\left(K^{p}\right)\left(U^{\bullet+1}\right) \\
& \simeq \operatorname{holim} M(J) \wedge T A F\left(K^{p}\right)_{\mathbb{F}_{p^{k}}} \\
& \simeq T A F\left(K^{p}\right)_{\mathbb{F}_{p^{k}}, K(n) .}
\end{aligned}
$$

Since the coherent sheaf $g_{c o h}^{*} \omega^{\otimes t}$ satisfies étale descent, the cohomology of the cosimplicial abelian group $\left(g_{c o h}^{*} \omega^{\otimes t}\right)\left(\widehat{U}^{\bullet+1}\right)$ is given by

$$
\pi^{s}\left(g_{c o h}^{*} \omega^{\otimes t}\right)\left(\widehat{U}^{\bullet+1}\right)= \begin{cases}\left(g_{c o h}^{*} \omega^{\otimes t}\right)\left(\left(S h\left(K^{p}\right)_{\mathbb{F}_{p^{k}}}\right)_{I_{n}}^{\wedge}\right) & s=0, \\ 0 & s \neq 0 .\end{cases}
$$

We therefore deduce that the map

$$
\prod_{i \in I\left(K^{p}, k\right)} E_{\overline{\mathbb{G}}_{i}} \rightarrow \operatorname{holim}_{\Delta} \prod_{i \in I\left(K^{p}, k, \bullet+1\right)} E_{\overline{\mathbb{G}_{\bullet}+1, i}}
$$

is an equivalence. The equivalence (14.4.7) therefore gives an equivalence

$$
\operatorname{TAF}\left(K^{p}\right)_{\mathbb{F}_{p^{k}}, K(n)} \stackrel{\simeq}{\longrightarrow} \prod_{i \in I\left(K^{p}, k\right)} E_{\overline{\mathbb{G}}_{i}} .
$$

Let $H_{n}$ be the height $n$ Honda formal group over $\overline{\mathbb{F}}_{p}$. Let $E_{n}=E_{H_{n}}$ denote the associated Morava E-theory. 
Corollary 14.4.8. Assume that $\operatorname{Sh}\left(K^{p}\right)^{[n]}$ is a scheme. There is an equivalence

$$
\operatorname{TAF}\left(K^{p}\right)_{\overline{\mathbb{F}}_{p}, K(n)} \simeq \prod_{\Gamma \backslash G U^{1}\left(\mathbb{A}^{p, \infty}\right) / K^{p}} E_{n} .
$$

Proof. Let $k$ be sufficiently large so that

$$
\operatorname{Sh}\left(K^{p}\right)^{[n]}\left(\mathbb{F}_{p^{k}}\right)=\operatorname{Sh}\left(K^{p}\right)^{[n]}\left(\overline{\mathbb{F}}_{p}\right) \cong \Gamma \backslash G U^{1}\left(\mathbb{A}^{p, \infty}\right) / K^{p} .
$$

(The last isomorphism is Corollary 14.3.4.) Then there is an isomorphism:

$$
\operatorname{Sh}\left(K^{p}\right)^{[n]} \cong \coprod_{\Gamma \backslash G U^{1}\left(\mathbb{A}^{p}, \infty\right) / K^{p}} \operatorname{Spec}\left(\mathbb{F}_{p^{k}}\right) .
$$

Then, by Proposition 14.4.6, there is an equivalence

$$
T A F\left(K^{p}\right)_{\mathbb{F}_{p^{k}}, K(n)} \stackrel{\simeq}{\rightarrow} \prod_{i \in \Gamma \backslash G U^{1}\left(\mathbb{A}^{p, \infty}\right) / K^{p}} E_{\left(\overline{\mathbb{G}}_{i}, \mathbb{F}_{p^{k}}\right)} .
$$

Taking the colimit over $k$ and $K(n)$-localizing gives an equivalence

$$
\left.\left(\underset{k}{\lim } T A F\left(K^{p}\right)_{\mathbb{F}_{p^{k}}, K(n)}\right)_{K(n)} \stackrel{\simeq}{\longrightarrow} \prod_{i \in \Gamma \backslash G U^{1}\left(\mathbb{A}^{p}, \infty\right) / K^{p}} \underset{k}{\left(\lim _{k}\right.} E_{\left(\overline{\mathbb{G}}_{i}, \mathbb{F}_{p^{k}}\right)}\right)_{K(n)} .
$$

Because $K(n)$-equivalences are preserved under colimits, we have

$$
\begin{aligned}
\left(\underset{k}{\lim } T A F\left(K^{p}\right)_{\mathbb{F}_{p^{k}}, K(n)}\right)_{K(n)} & \simeq\left(\underset{k}{\lim } T A F\left(K^{p}\right)_{\mathbb{F}_{p^{k}}}\right)_{K(n)} \\
& \simeq T A F\left(K^{p}\right)_{\overline{\mathbb{F}}_{p}, K(n)} .
\end{aligned}
$$

Each height $n$ formal group $\overline{\mathbb{G}}_{i}$ is isomorphic to $H_{n}$ over $\overline{\mathbb{F}}_{p}$. Therefore, using Proposition 14.4.5 and the functoriality of the Goerss-Hopkins-Miller theorem (Theorem 8.1.3), there are equivalences

$$
\begin{aligned}
\left.{\underset{k}{l i m}}_{\left(\overline{\mathbb{G}}_{i}, \overline{\mathbb{F}}_{p}^{k}\right)}\right)_{K(n)} & \simeq \operatorname{holim} \lim _{J} E_{\left(\overline{\mathbb{G}}_{i}, \overline{\mathbb{F}}_{p}^{k}\right)} \wedge M(J) \\
& \simeq E_{n} .
\end{aligned}
$$

The equivalence (14.4.9) therefore gives an equivalence

$$
T A F\left(K^{p}\right)_{\overline{\mathbb{F}}_{p}, K(n)} \stackrel{\simeq}{\longrightarrow} \prod_{\Gamma \backslash G U^{1}\left(\mathbb{A}^{p, \infty}\right) / K^{p}} E_{n} .
$$

Taking Gal-homotopy fixed points, we arrive at the following.

Corollary 14.4.10. Assume that $\operatorname{Sh}\left(K^{p}\right)^{[n]}$ is a scheme. There is an equivalence

$$
T A F\left(K^{p}\right)_{K(n)} \simeq\left(\prod_{\Gamma \backslash G U^{1}\left(\mathbb{A}^{p, \infty}\right) / K^{p}} E_{n}\right)^{h G a l} .
$$

Remark 14.4.11. In Corollary 14.4.10, the action of the Galois group Gal is typically non-trivial on the index set

$$
\operatorname{Sh}\left(K^{p}\right)^{[n]}\left(\overline{\mathbb{F}}_{p}\right) \cong \Gamma \backslash G U^{1}\left(\mathbb{A}^{p, \infty}\right) / K^{p}
$$


and the action of Gal on the components $E_{n}$ is not the typical one. This is because the equivalence

$$
\left(\underset{k}{\left(\lim _{(}\right.} E_{\left(\overline{\mathbb{G}}_{i}, \overline{\mathbb{F}}_{p}^{k}\right)}\right)_{K(n)} \simeq E_{n}
$$

appearing in the proof of Corollary 14.4.8 is not Gal-equivariant; the formal group $\mathbb{G}_{i}$ may not even be defined over $\mathbb{F}_{p}$.

\section{5. $K(n)$-local $Q_{U}$}

Let $G$ and $G^{1}$ denote the groups $G U\left(\mathbb{A}^{p, \infty}\right)$ and $G U^{1}\left(\mathbb{A}^{p, \infty}\right)$, respectively. In Section 11.1, using a construction described in Section 10.6, we produced a fibrant smooth $G$-spectrum $V=V_{G U}$ such that

$$
V \simeq \underset{\mathrm{K}^{p}}{\lim } \operatorname{TAF}\left(K^{p}\right) .
$$

The colimit is taken over compact open subgroups $K^{p}$ of $G$. There is a cofinal collection of subgroups $K^{p}$ such that $S h\left(K^{p}\right)^{[n]}$ is a scheme. Let $V_{\mathbb{F}_{p}}$ be a fibrant smooth $G \times G a l$-spectrum such that

$$
V_{\mathbb{F}_{p}} \simeq \underset{\lim ^{p}, k}{ } \operatorname{TAF}\left(K^{p}\right)_{\overline{\mathbb{F}}_{p^{k}}} .
$$

Let $\mathrm{Sp}_{\Gamma}$ be the category of $\Gamma$-equivariant spectra, with the injective model structure. The cofibrations and weak equivalences in this model structure are detected on the underlying category of spectra. Consider the adjoint pair $\left(\operatorname{Res}_{\Gamma}^{G^{1}}, \operatorname{Map}_{\Gamma}\left(G^{1},-\right)^{s m}\right)$ :

$$
\operatorname{Res}_{\Gamma}^{G^{1}}: \operatorname{Sp}_{G^{1}}^{s m} \leftrightarrows \operatorname{Sp}_{\Gamma}: \operatorname{Map}_{\Gamma}\left(G^{1},-\right)^{s m}
$$

defined by

$$
\begin{aligned}
\operatorname{Res}_{\Gamma}^{G^{1}} X & =X, \\
\operatorname{Map}_{\Gamma}\left(G^{1}, Y\right)^{s m} & =\underset{H \underset{\leq_{o} G^{1}}{\longrightarrow}}{\lim _{\Gamma}\left(G^{1} / H, Y\right) .}
\end{aligned}
$$

The $G^{1}$-action on $\operatorname{Map}_{\Gamma}\left(G^{1}, Y\right)^{s m}$ is by precomposition with right multiplication.

The following double coset formula is very useful.

Lemma 14.5.1. Let $H$ and $K$ be subgroups of a group $G$. Let $Y$ be an $H$-spectrum. There is an isomorphism

$$
\operatorname{Map}_{H}(G / K, Y) \cong \prod_{[g] \in H \backslash G / K} Y^{H \cap g K g^{-1}} .
$$

Lemma 14.5.2. Let $Y$ be a $\Gamma$-spectrum. Suppose that $K^{p}$ be sufficiently small so that $\operatorname{Sh}\left(K^{p}\right)^{[n]}$ is a scheme. Then there is an isomorphism

$$
\operatorname{Map}_{\Gamma}\left(G^{1} / K^{p}, Y\right) \cong \prod_{\Gamma \backslash G^{1} / K^{p}} Y .
$$

Proof. By Proposition 14.1.2, for every element $g \in G^{1}$, the group

$$
\Gamma\left(g K^{p} g^{-1}\right)=\Gamma \cap g K^{p} g^{-1}
$$


is the automorphism group of a $\overline{\mathbb{F}}_{p}$-point of the stack $S h\left(K^{p}\right)^{[n]}$. Because these automorphism groups are trivial, the lemma follows immediately from Lemma 14.5.1.

\section{LEMMA 14.5.3.}

(1) The functors $\left(\operatorname{Res}_{\Gamma}^{G^{1}}, \operatorname{Map}_{\Gamma}\left(G^{1},-\right)^{\text {sm }}\right)$ form a Quillen pair.

(2) The functor $\operatorname{Map}_{\Gamma}\left(G^{1},-\right)^{\text {sm }}$ takes fibrant $\Gamma$-spectra to fibrant smooth $G^{1}$ spectra.

(3) The functor $\operatorname{Map}_{\Gamma}\left(G^{1},-\right)^{\text {sm }}$ preserves all weak equivalences.

Proof. Using Lemma 10.3.4, it is clear that the functor $\operatorname{Res}_{\Gamma}^{G^{1}}$ preserves cofibrations and weak equivalences, and this proves (1). Statement (2) is an immediate consequence of statement (1). Because there is a natural isomorphism

$$
\operatorname{Map}_{\Gamma}\left(G^{1},-\right)^{s m} \cong \underset{K^{p}}{\lim _{\longrightarrow}} \operatorname{Map}_{\Gamma}\left(G^{1} / K^{p},-\right),
$$

where the colimit is taken over $K^{p}$ such that $S h\left(K^{p}\right)^{[n]}$ is a scheme, Statement (3) follows from Lemma 14.5.2.

Fix an $\overline{\mathbb{F}}_{p^{-}}$-point $(A, i, \lambda,[\eta])$ of $S h\left(K^{p}\right)^{[n]}$ (where $K^{p}$ is some compact open subgroup of $\left.G^{1}\right)$. Fix an isomorphism $\alpha: \epsilon A(u) \cong H_{n}$, where $H_{n}$ is the Honda height $n$ formal group. The action of $\Gamma$ on $\epsilon A(u)$ and the isomorphism $\alpha$ gives an action of $\Gamma$ on the spectrum $E_{n}$. The following proposition is immediate from Corollary 14.4.8 and Lemma 14.5.2.

Proposition 14.5.4. There is an $K(n)$-equivalence

$$
V_{\overline{\mathbb{F}}_{p}} \rightarrow \operatorname{Map}_{\Gamma}\left(G^{1}, E_{n}\right)^{s m}
$$

of smooth $G^{1} \times$ Gal-spectra.

We now are able to prove our main $K(n)$-local result.

THEOREM 14.5.5. Let $U$ be an open subgroup of $G^{1}$. There is an equivalence

$$
\left(V^{h U}\right)_{K(n)} \stackrel{\simeq}{\longrightarrow}\left(\prod_{[g] \in \Gamma \backslash G^{1} / U} E_{n}^{h \Gamma\left(g U g^{-1}\right)}\right)^{h G a l} .
$$

Proof. It suffices to prove that there is a Gal-equivariant equivalence

$$
\left(V_{\mathbb{F}_{p}}^{h U}\right)_{K(n)} \stackrel{\simeq}{\longrightarrow} \prod_{[g] \in \Gamma \backslash G^{1} / U} E_{n}^{h \Gamma\left(g U g^{-1}\right)} .
$$

The result is then obtained by taking homotopy fixed points with respect to Fr $\epsilon$ Gal (Lemma 14.4.4). By Proposition 14.4.5, a map between $E(n)$-local spectra which is an $M(J)$-equivalence for every $J$ is a $K(n)$-equivalence (it actually suffices to only check this for a single $J)$. Since localization with respect to $E(n)$ is smashing, colimits of $E(n)$-local spectra are $E(n)$-local. Therefore, the spectra $V_{\mathbb{F}_{p}}$ and 
$\operatorname{Map}_{\Gamma}\left(G^{1}, E_{n}\right)^{s m}$ are $E(n)$-local. Since $M(J)$ is finite, Proposition 14.5.4 implies that there is a Gal-equivariant $K(n)$-local equivalence

$$
V_{\mathbb{F}_{p}}^{h U} \rightarrow\left(\operatorname{Map}_{\Gamma}\left(G^{1}, E_{n}\right)^{s m}\right)^{h U} .
$$

Let $E_{n}^{\prime}$ be a fibrant replacement for $E_{n}$ in the category of $\Gamma$-equivariant spectra. By Lemma 14.5.3, the spectrum $\operatorname{Map}_{\Gamma}\left(G^{1}, E_{n}\right)^{s m}$ is fibrant as a smooth $G^{1}$-spectrum, and the map

is a weak equivalence.

$$
\operatorname{Map}_{\Gamma}\left(G^{1}, E_{n}\right)^{s m} \rightarrow \operatorname{Map}_{\Gamma}\left(G^{1}, E_{n}^{\prime}\right)^{s m}
$$

Using Corollary 10.5.5 and Lemma 14.5.1, we have the following sequence of equivalences:

$$
\begin{aligned}
\left(\operatorname{Map}_{\Gamma}\left(G^{1}, E_{n}\right)^{s m}\right)^{h U} & \simeq\left(\operatorname{Map}_{\Gamma}\left(G^{1}, E_{n}^{\prime}\right)^{s m}\right)^{U} \\
& \cong \operatorname{Map}_{\Gamma}\left(G^{1} / U, E_{n}^{\prime}\right) \\
& \cong \prod_{[g] \in \Gamma \backslash G^{1} / U}\left(E_{n}^{\prime}\right)^{\Gamma\left(g U g^{-1}\right)} \\
& \simeq \prod_{[g] \in \Gamma \backslash G^{1} / U}\left(E_{n}\right)^{h \Gamma\left(g U g^{-1}\right)} .
\end{aligned}
$$

Specializing to the cases of $U=K^{p}$ and $U=K^{p, S}$, we have the following corollary. Corollary 14.5.6. Let $K^{p}$ be an open compact subgroup of $G U\left(\mathbb{A}^{p, \infty}\right)$. There are equivalences

$$
\begin{aligned}
T A F\left(K^{p}\right)_{K(n)} & \simeq\left(\prod_{[g] \in \Gamma \backslash G U^{1}\left(\mathbb{A}^{p, \infty}\right) / K^{p}} E_{n}^{h \Gamma\left(g K^{p} g^{-1}\right)}\right)^{h G a l}, \\
Q_{U}\left(K^{p, S}\right)_{K(n)} & \simeq\left(\prod_{[g] \in \Gamma \backslash G U^{1}\left(\mathbb{A}^{p, S, \infty}\right) / K^{p, S}} E_{n}^{h \Gamma\left(g K^{p, S} g^{-1}\right)}\right)^{h G a l} .
\end{aligned}
$$




\section{CHAPTER 15}

\section{Example: chromatic level 1}

In this chapter we provide some analysis of the spectrum $T A F$ and the associated homotopy fixed point spectrum at chromatic filtration 1 . In particular, we find that these spectra are closely related to the $K(1)$-local sphere.

\subsection{Unit groups and the $K(1)$-local sphere}

In this section, we indicate how we can recover a description of the $K(1)$-local sphere by making use of units in a field extension of the rationals.

Let $E_{1}$ be the Lubin-Tate spectrum whose homotopy groups are $W\left(\overline{\mathbb{F}}_{p}\right)\left[u^{ \pm 1}\right]$ with $|u|=2$. We regard $E_{1}$ as being the Hopkins-Miller spectrum associated to the formal multiplicative group $\widehat{\mathbb{G}}_{m}$ over $\overline{\mathbb{F}}_{p}$. Since the formal group $\widehat{\mathbb{G}}_{m}$ is defined over $\mathbb{F}_{p}$, the spectrum $E_{1}$ possesses an action of

$$
\text { Gal }=\operatorname{Gal}\left(\overline{\mathbb{F}}_{p} / \mathbb{F}_{p}\right)
$$

by $E_{\infty}$ ring maps. The work of Goerss and Hopkins [GH04] specializes to prove that there is an isomorphism

$$
\mathbb{G}_{1} \cong \operatorname{Aut}_{E_{\infty}}\left(E_{1}\right)
$$

where

$$
\mathbb{G}_{1}=\mathbb{S}_{1} \times G a l \cong \mathbb{Z}_{p}^{\times} \times \widehat{\mathbb{Z}} .
$$

Specifically, for $k \in \mathbb{Z}_{p}^{\times}$, there is an Adams operation $\psi^{k}: E_{1} \rightarrow E_{1}$ such that $\psi_{*}^{k}(u)=k u$. The Gal-homotopy fixed points of the spectrum $E_{1}$ is the spectrum $K U_{p}$, the $p$-completion of the complex $K$-theory spectrum. The action of $\mathbb{Z}_{p}^{\times}$on $E_{1}$ descends to an action on $K U_{p}$, and the Adams operations $\psi_{*}^{k}$ restrict to give the usual Adams operations on $p$-adic $K$-theory.

The product decomposition

$$
\operatorname{Aut}_{E_{\infty}}\left(E_{1}\right) \cong \mathbb{S}_{1} \times G a l
$$

is not canonical. Rather, there is a canonical short exact sequence of profinite groups

$$
1 \rightarrow \mathbb{S}_{1} \rightarrow \operatorname{Aut}_{E_{\infty}}\left(E_{1}\right) \rightarrow \text { Gal } \rightarrow 1
$$

and the choice of formal group $\widehat{\mathbb{G}}_{m}$ over $\mathbb{F}_{p}$ gives rise to a splitting. More generally, Morava [Mor89] studied forms of $K$-theory: $p$-complete ring spectra $K^{\prime}$ such that there exists an isomorphism of multiplicative cohomology theories

$$
K U_{p}^{*}(-) \otimes_{\mathbb{Z}_{p}} W\left(\overline{\mathbb{F}}_{p}\right) \stackrel{\cong}{\longrightarrow} K^{\prime *}(-) \otimes_{\mathbb{Z}_{p}} W\left(\overline{\mathbb{F}}_{p}\right) .
$$


Morava showed that there was an isomorphism

$$
\left\{\text { forms of } K U_{p}\right\} \cong H_{c}^{1}\left(G a l ; \mathbb{Z}_{p}^{\times}\right) \cong \mathbb{Z}_{p}^{\times} .
$$

Using Goerss-Hopkins-Miller theory, one can strengthen Morava's theorem to prove that there is an isomorphism

$$
\left\{E_{\infty} \text { forms of } K U_{p}\right\} \stackrel{\cong}{\rightrightarrows} H_{c}^{1}\left(G a l ; \mathbb{Z}_{p}^{\times}\right) .
$$

Given a Galois cohomology class

$$
\alpha \in H^{1}\left(G a l ; \mathbb{Z}_{p}^{\times}\right)
$$

we may regard it as giving a splitting of the short exact sequence (15.1.1), and thus an inclusion

$$
\iota_{\alpha}: G a l \hookrightarrow \operatorname{Aut}_{E_{\infty}}\left(E_{1}\right) .
$$

The $E_{\infty}$-form $K_{\alpha}$ associated to the cohomology class $\alpha$ is given as the homotopy fixed points of the new Galois action on $E_{1}$ induced by the inclusion $\iota_{\alpha}$ :

$$
K_{\alpha}=E_{1}^{h_{\alpha} G a l} .
$$

The $K(1)$-local sphere is known to be homotopy equivalent to the fiber of the map $\psi^{k}-1: K O_{p} \rightarrow K O_{p}$, where $k$ is any topological generator of the group $\mathbb{Z}_{p}^{\times} /\{ \pm 1\}$. Using the equivalence of Devinatz-Hopkins [DH04]

$$
S_{K(1)} \stackrel{\simeq}{\longrightarrow} E_{1}^{h \mathbb{G}_{1}}
$$

and (15.1.2), we can substitute the $K O_{p}$-spectrum with the fixed points of any form of $K$-theory to give a fiber sequence

$$
S_{K(1)} \rightarrow K_{\alpha}^{h\{ \pm 1\}} \stackrel{\psi^{k}-1}{\longrightarrow} K_{\alpha}^{h\{ \pm 1\}} .
$$

Let $F$ be a quadratic imaginary extension field of $\mathbb{Q}$, and let $p$ be a prime of $\mathbb{Q}$ that splits in $F$ as $u u^{c}$. This corresponds to the existence of an embedding $u: F \rightarrow \mathbb{Q}_{p}$. If $\mathcal{O}_{F}$ is the ring of integers of $F$, there is a corresponding embedding $u: \mathcal{O}_{F} \rightarrow \mathbb{Z}_{p}$.

The Dirichlet unit theorem tells us that the unit group of $\mathcal{O}_{F}$ is finite. As the extension is quadratic, there are only three possibilities. If $F=\mathbb{Q}(i), \mathcal{O}_{F}^{\times}$is the group of fourth roots of unity. If $F=\mathbb{Q}(\omega)$, where $\omega$ is a third root of unity, then $\mathcal{O}_{F}^{\times}$is the group of sixth roots of unity. In either of these two exceptional cases, the primes 2 and 3 are nonsplit. In any other case, $\mathcal{O}_{F}^{\times}$is $\{ \pm 1\}$.

Fix a form of $K$-theory $K_{\alpha}$. The map $\mathcal{O}_{F} \rightarrow \mathbb{Z}_{p}$ gives an action of the (finite) unit group of $\mathcal{O}_{F}$ on $K_{\alpha}$. If $p \neq 2$, this action factors through the action of the roots of unity $\mu_{p-1} \subset \mathbb{Z}_{p}^{\times}$, and the homotopy fixed point set of the action of $\mathcal{O}_{F}^{\times}$on $\mathbb{Z}_{p}$ is a wedge of suspensions (forms of) of Adams summands. If $p=2$, then $\mathcal{O}_{F}^{\times}=\{ \pm 1\}$, and the homotopy fixed point set is $K_{\alpha}^{\{ \pm 1\}}$, additively equivalent to $\mathrm{KO}_{2}$.

However, the image of $\mathcal{O}_{F}$ in $\mathbb{Z}_{p}$ contains more units than merely this finite subgroup. Let $S_{F}$ be a finite set of primes of $\mathcal{O}_{F}$ that do not divide $p$. Then there is an extension map $S_{F}^{-1} \mathcal{O}_{F} \rightarrow \mathbb{Z}_{p}$, and an action of $\left(S_{F}^{-1} \mathcal{O}_{F}\right)^{\times}$on $K_{\alpha}$. 
Let $\mathrm{Cl}(F)$ denote the ideal class group of $F$. There is an exact sequence

$$
0 \rightarrow \mathcal{O}_{F}^{\times} \rightarrow\left(S_{F}^{-1} \mathcal{O}_{F}\right)^{\times} \stackrel{\oplus \nu}{\longrightarrow} \bigoplus_{S_{F}} \mathbb{Z} \rightarrow \mathrm{Cl}(F) \rightarrow \mathrm{Cl}(F) /\left\langle S_{F}\right\rangle \rightarrow 0 .
$$

The class group of a number field is finite, so $\left(S_{F}^{-1} \mathcal{O}_{F}\right)^{\times}$is isomorphic to $\mathcal{O}_{F}^{\times} \oplus \mathbb{Z}^{\left|S_{F}\right|}$.

In particular, if we invert only 1 prime $w\left(\right.$ so $\left|S_{F}\right|=1$ ), there exists a smallest integer $d$ such that $w^{d}=(\kappa)$ as ideals for some $\kappa \in \mathcal{O}_{F}$. The homotopy fixed point set of $K_{\alpha}$ under the action of $\left(S_{F}^{-1} \mathcal{O}_{F}\right)^{\times}$is the spectrum

$$
\left(K_{\alpha}^{h \mathcal{O}_{F}^{\times}}\right)^{h\langle\kappa\rangle}
$$

or equivalently the homotopy fiber of the map $[\kappa]-1: K_{\alpha}^{h \mathcal{O}_{F}^{\times}} \rightarrow K_{\alpha}^{h \mathcal{O}_{F}^{\times}}$. If $\kappa$ is a topological generator of $\mathbb{Z}_{p}^{\times} / \mathcal{O}_{F}^{\times}$, the resulting homotopy fixed point spectrum is, in fact, the $K(1)$-local sphere.

Global class field theory and the Chebotarev density theorem show that prime ideals of $F$ are uniformly distributed in the class group, and principal prime ideals are uniformly distributed in any congruence condition. Therefore, it is always possible to pick a principal prime ideal $(\kappa)$ of $\mathcal{O}_{F}$ such that $\kappa$ maps to a topological generator of the (topologically cyclic) group $\mathbb{Z}_{p}^{\times} /\{ \pm 1\}$.

One the other hand, suppose one inverts a set $S_{F}$ of primes with $\left|S_{F}\right|>1$. Let $H$ be the closure of the image of $\left(S_{F}^{-1} \mathcal{O}_{F}\right)^{\times}$in $\mathbb{Z}_{p}^{\times} / \mathcal{O}_{F}^{\times}$. The group $H$ is cyclic, and hence there is a decomposition

$$
\left(S_{F}^{-1} \mathcal{O}_{F}\right)^{\times} \cong \mathcal{O}_{F}^{\times} \times\langle x\rangle \times \mathbb{Z}^{\left|S_{F}\right|-1}
$$

such that the image of $x$ is a topological generator of $H$. Then there is a decomposition of the homotopy fixed point set of $K_{\alpha}$ under $\left(S_{F}^{-1} \mathcal{O}_{F}\right)^{\times}$as

$$
\left(K_{\alpha}^{h \mathcal{O}_{F}^{\times} \times\langle x\rangle}\right)^{h \mathbb{Z}^{\left|S_{F}\right|-1}}
$$

However, $\mathbb{Z}^{\left|S_{F}\right|-1}$ acts through $H$, which acts trivially on the homotopy fixed point spectrum. Therefore, the homotopy fixed point spectrum is the function spectrum

$$
F\left(\left(B \mathbb{Z}^{\left|S_{F}\right|-1}\right)_{+}, K_{\alpha}^{h \mathcal{O}_{F}^{\times} \times\langle x\rangle}\right),
$$

and therefore decomposes as a wedge of suspensions of $K_{\alpha}^{h \mathcal{O}_{F}^{\times} \times\langle x\rangle}$.

\subsection{Topological automorphic forms in chromatic filtration 1}

We now analyze the homotopy fixed point spectra associated to height 1 Shimura varieties. In this case, much of the required data becomes redundant.

Let $F$ be a quadratic imaginary extension of $\mathbb{Q}$ in which $p$ splits. The central simple algebra over $F$ of degree $n^{2}$ must be $F$ itself in this case, and the maximal order must be $\mathcal{O}_{F}$.

A $\mathbb{Q}$-valued nondegenerate hermitian alternating form on $F$ is of the form

$$
(x, y)=\operatorname{Tr}_{F / \mathbb{Q}}\left(x \beta y^{*}\right)
$$


for some nonzero $\beta \in F$ such that $\beta^{*}=-\beta$, i.e. $\beta$ is purely imaginary. However, any two such $\beta$ differ by a scalar multiple, and so there is a unique similitude class of such pairings on $F$. The involution induced on $F$ is, of necessity, complex conjugation.

The group scheme $G U$ satisfies $G U(R) \cong(R \otimes F)^{\times}$, and the group scheme $U$ is the associated unitary group. There are isomorphisms

$$
\begin{aligned}
G U\left(\mathbb{A}^{p, \infty}\right) & \cong\left(\mathbb{A}_{F}^{u, u^{c}, \infty}\right)^{\times} \\
& \cong \prod_{w \backslash p}^{\prime} F_{w}^{\times} \\
G U^{1}\left(\mathbb{A}^{p, \infty}\right) & \cong\left(\prod_{\ell \text { split }}^{\prime} \mathbb{Q}_{\ell}^{\times} \times \mathbb{Z}_{\ell}^{\times}\right) \times\left(\prod_{\ell \text { nonsplit }} \mathcal{O}_{F, \ell}^{\times}\right)
\end{aligned}
$$

There is a unique maximal open compact subgroup of $G U\left(\mathbb{A}^{p, \infty}\right)$, namely the group of units

$$
K_{0}^{p}=\prod_{w \chi p} \mathcal{O}_{F, w}^{\times}
$$

Every elliptic curve $E$ comes equipped with a canonical weak polarization which is an isomorphism, and any other weak polarization differs by multiplication by a rational number. A map $\mathcal{O}_{F,(p)} \rightarrow \operatorname{End}(E)_{(p)}$ is equivalent to a map $F \rightarrow \operatorname{End}^{0}(E)$, as follows. The existence of the map implies that the elliptic curve is ordinary, as $p$ is split in $F$. Using the pullback

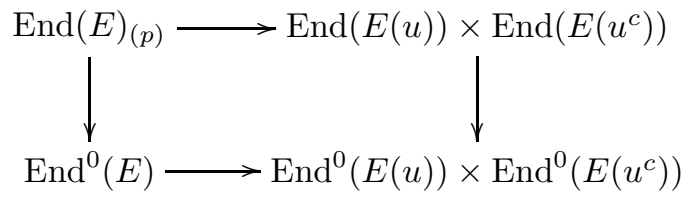

and the isomorphism

$$
\operatorname{End}(E(u)) \times \operatorname{End}\left(E\left(u^{c}\right)\right) \cong \mathbb{Z}_{p} \times \mathbb{Z}_{p}
$$

we see the $\operatorname{map} \mathcal{O}_{F,(p)} \rightarrow \operatorname{End}^{0}(E)$ factors through $\operatorname{End}(E)_{(p)}$.

There are two choices for the map $\mathcal{O}_{F,(p)} \rightarrow \operatorname{End}(E)_{(p)}$ that differ by complex conjugation. However, there exists a unique choice such that the corresponding summand $E(u)$ is the formal summand and $E\left(u^{c}\right)$ is the étale summand.

Therefore, the Shimura variety $S h\left(K_{0}^{p}\right)$ associated to this data classifies elliptic curves with complex multiplication by $F$. The automorphism group of any such object is the unit group $\mathcal{O}_{F}^{\times}$. The moduli of elliptic curves with complex multiplication by $F$ breaks up geometrically as a disjoint union indexed by the class group of $F$, as follows.

$$
\operatorname{Sh}\left(K_{0}^{p}\right) \times_{\operatorname{Spec}\left(\mathbb{Z}_{p}\right)} \operatorname{Spec}\left(W\left(\overline{\mathbb{F}}_{p}\right)\right) \cong \coprod_{\operatorname{Cl}(F)} \operatorname{Spec}\left(\mathbb{W}\left(\overline{\mathbb{F}}_{p}\right)\right) / / \mathcal{O}_{F}^{\times}
$$

Here // denotes the stack quotient by a trivial group action. 
The main theorem of complex multiplication for elliptic curves has the following consequence. Let $K$ be the Hilbert class field of $F$, i.e. the unique totally unramified extension of $F$, which has Galois group $\mathrm{Cl}(F)$, and let $\mathcal{O}_{K}$ be its ring of integers. Then we have the following isomorphism:

$$
\operatorname{Sh}\left(K_{0}^{p}\right) \cong \operatorname{Spec}\left(\mathcal{O}_{K, u}\right) / / \mathcal{O}_{F}^{\times} \cong \coprod_{\mathfrak{P} \mid u} \operatorname{Spec}\left(\mathcal{O}_{K, \mathfrak{P}}\right) / / \mathcal{O}_{F}^{\times} .
$$

Here the coproduct is over primes $\mathfrak{P}$ of $K$ dividing $u$. Isomorphism (15.2.1) follows from the fact that there is a map

$$
\mathcal{O}_{F}[j(E)] \rightarrow \mathcal{O}_{K}
$$

which an isomorphism at $p$, where $j(E)$ is the $j$-invariant of any elliptic curve with complex multiplication by $F$.

The spectrum of topological automorphic forms associated to the Shimura variety is the spectrum

$$
\operatorname{TAF}\left(K_{0}^{p}\right) \simeq\left(\prod_{\mathrm{Cl}(F)} E_{1}^{h \mathcal{O}_{F}^{\times}}\right)^{h G a l}
$$

The spectrum of topological automorphic forms can be expressed as follows. For a prime $\mathfrak{P}$ of $K$ dividing $u$, let $D_{\mathfrak{P}}$ be the decomposition group, and let $f$ denote its order. Under the isomorphism $\operatorname{Gal}(K / F) \cong \mathrm{Cl}(F), D_{\mathfrak{P}}$ is the subgroup generated by $u$. Because $K$ is unramified over $F$, the integer $f$ is equal to the residue class degree of $\mathfrak{P}$ over $u$. In particular, there are isomorphisms

$$
\mathcal{O}_{K, u} \cong \prod_{\mathfrak{P} \mid u} \mathcal{O}_{K, \mathfrak{P}} \cong \prod_{\mathrm{Cl}(F) / D_{\mathfrak{P}}} W\left(\mathbb{F}_{p^{j}}\right) .
$$

We deduce that all of the $\overline{\mathbb{F}}_{p}$ points of $S h\left(K_{0}^{p}\right)$ are defined over $\mathbb{F}_{p^{j}}$, and that, for each prime $\mathfrak{P}$, there exists an elliptic curve $E_{\mathfrak{P}} / \mathbb{F}_{p^{j}}$ with complex multiplication by $F$, such that the isomorphism classes of $S h\left(K_{0}^{p}\right)\left(\overline{\mathbb{F}}_{p}\right)$ are represented by the set of elliptic curves

$$
\left\{E_{\mathfrak{P}}^{\left(p^{i}\right)}: \mathfrak{P} \mid u, 0 \leq i<f\right\}
$$

(where $E_{\mathfrak{P}}^{\left(p^{i}\right)}$ denotes the pullback of $E_{\mathfrak{P}}$ over the $i$ th power of the Frobenius).

Let $K\left(E_{\mathfrak{P}}\right)$ be the form of $K U_{p} \otimes_{\mathbb{Z}_{p}} W\left(\mathbb{F}_{p^{j}}\right)$ corresponding to the unique deformation of the height 1 formal group $\widehat{E}_{\mathfrak{P}}$ over $W\left(\mathbb{F}_{p^{j}}\right)$. The homotopy of $K\left(E_{\mathfrak{P}}\right)$ is given by

$$
\pi_{*} K\left(E_{\mathfrak{P}}\right) \cong \mathcal{O}_{K, \mathfrak{P}}\left[u^{ \pm 1}\right] \cong W\left(\mathbb{F}_{p^{f}}\right)\left[u^{ \pm 1}\right] .
$$

We have the following:

$$
T A F\left(K_{0}^{p}\right) \simeq \prod_{\mathfrak{P} \mid u} K\left(E_{\mathfrak{P}}\right)^{h \mathcal{O}_{F}^{\times}}
$$

Let $S$ be a finite set of primes of $\mathbb{Q}$. Let $S_{F}$ be the collection of primes of $F$ dividing the primes in $S$. 
Case where the primes of $S$ do not split in $F$. Suppose that each of the primes in $S$ does not split in $F$. Then there is an equivalence

$$
Q_{G U}\left(K_{0}^{p, S}\right)=\left(\prod_{\mathrm{Cl}(F) /\langle S\rangle} E_{1}^{h\left(S^{-1} \mathcal{O}_{F}\right)^{\times}}\right)^{h G a l}
$$

In particular, if $\ell$ is chosen to be a topological generator of $\mathbb{Z}_{p}^{\times}$, and is inert in $F$, then there is an equivalence

$$
Q_{G U}\left(K_{0}^{p, \ell}\right) \stackrel{\simeq}{\longrightarrow} \prod_{\mathrm{Cl}(F) / D_{\mathfrak{P}}} S_{K(1), \mathbb{F}_{p} f}
$$

Here, $S_{K(1), \mathbb{F}_{p f}}$ is the Galois extension of $S_{K(1)}=\left(E_{1}^{h \mathbb{S}_{1}}\right)^{h G a l_{\mathbb{F}_{p}}}$ given by the fixed point spectrum

$$
S_{K(1), \mathbb{F}_{p} f}=\left(E_{1}^{h \mathbb{S}_{1}}\right)^{h G a l_{\mathbb{F}_{p} f}} .
$$

The building $\mathcal{B}(G U)$ for $G U\left(\mathbb{Q}_{\ell}\right)=\mathcal{O}_{F, \ell}^{\times}$is homeomorphic to the real line $\mathbb{R}$. The group $\mathcal{O}_{F, \ell}^{\times}$acts by translation by $\ell$-adic valuation. The resulting building decomposition is a product of $J$-fiber sequences

$$
Q_{G U}\left(K_{0}^{p, \ell}\right) \rightarrow \prod_{\mathfrak{P} \mid u} K\left(E_{\mathfrak{P}}\right)^{h \mathcal{O}_{F}^{\times}} \stackrel{\prod \psi^{\ell}-1}{\longrightarrow} \prod_{\mathfrak{P} \mid u} K\left(E_{\mathfrak{P}}\right)^{h \mathcal{O}_{F}^{\times}} .
$$

Case where the primes in $S$ split in $F$. Suppose that each of the primes in $S$ is split in $F$. Let $S_{F}^{\prime} \subset S_{F}$ be a set containing exactly one prime dividing $\ell$ for each $\ell \in S$. The group $\Gamma\left(K_{0}^{p, S}\right)$ is given by

$$
\Gamma\left(K_{0}^{p, S}\right)=\left(S^{-1} \mathcal{O}_{F}\right)^{N=1},
$$

the subgroup of $\left(S^{-1} \mathcal{O}_{F}\right)^{\times}$for which the norm $N=N_{F / \mathbb{Q}}$ is 1 . There is an exact sequence

$$
0 \rightarrow\left(S^{-1} \mathcal{O}_{F}\right)^{N=1} \rightarrow\left(S^{-1} \mathcal{O}_{F}\right)^{\times} \stackrel{\oplus \nu_{\ell} N}{\longrightarrow} \bigoplus_{S} \mathbb{Z} .
$$

It follows from (15.1.3) that there is an exact sequence

$$
0 \rightarrow \mathcal{O}_{F}^{\times} \rightarrow\left(S^{-1} \mathcal{O}_{F}\right)^{N=1} \stackrel{\oplus \nu_{w}}{\longrightarrow} \bigoplus_{w \in S_{F}^{\prime}} \mathbb{Z} \stackrel{\kappa}{\rightarrow} \mathrm{Cl}(F)
$$

where

$$
\kappa\left(\sum_{w \in S_{F}^{\prime}} n_{w}(w)\right)=\sum_{w \in S_{F}^{\prime}} n_{w}[w]-n_{w}\left[w^{c}\right] \in \mathrm{Cl}(F) .
$$

In particular, there is an isomorphism

$$
\Gamma\left(K_{0}^{p, S}\right) \cong \mathcal{O}_{F}^{\times} \times \mathbb{Z}^{|S|} .
$$

Following the techniques of Section 15.1, the closure $H$ of the image of $\Gamma\left(K_{0}^{p, S}\right)$ in $\mathbb{Z}_{p}^{\times} / \mathcal{O}_{F}^{\times}$is cyclic. Choosing a generator $x \in \Gamma\left(K_{0}^{p, S}\right)$ gives a decomposition

$$
\Gamma\left(K_{0}^{p, S}\right) \cong \mathcal{O}_{F}^{\times} \times\langle x\rangle \times \mathbb{Z}^{|S|-1} .
$$


We therefore have an equivalence

$$
Q_{U}\left(K_{0}^{p, S}\right) \simeq\left(\prod_{\mathrm{Cl}(F) /\left\langle S_{F}^{\prime}\right\rangle} F\left(B \mathbb{Z}_{+}^{|S|-1}, E_{1}^{h \mathcal{O}_{F}^{\times} \times\langle x\rangle}\right)\right)^{h G a l} .
$$

Assume now that $S$ consists of a single prime $\ell$ which splits as $w w^{c}$, such that:

(1) the ideal $w=(t)$ is principal,

(2) the element $q=t / t^{c} \in \Gamma\left(K_{0}^{p, \ell}\right) \subset \mathbb{Z}_{p}^{\times}$is a topological generator of $\mathbb{Z}_{p}^{\times} / \mathcal{O}_{F}^{\times}$.

Infinitely many such primes can be shown to exist, using class field theory and the Chebotarev density theorem. Condition (2) implies that $\Gamma\left(K_{0}^{p, \ell}\right)$ is dense in $\mathbb{Z}_{p}^{\times}$. Then there is an equivalence

$$
Q_{U}\left(K_{0}^{p, \ell}\right) \simeq \prod_{\mathrm{Cl}(F) / D_{\mathfrak{P}}} S_{K(1), \mathbb{F}_{p} f} .
$$

The building $\mathcal{B}(U)$ for the group

$$
U\left(\mathbb{Q}_{\ell}\right)=\left(F_{\ell}\right)^{N=1} \cong F_{w}^{\times}
$$

is homeomorphic to $\mathbb{R}$. An element $g \in U\left(\mathbb{Q}_{\ell}\right)$ acts on $\mathcal{B}(U)$ by translation by $\nu_{w}(g)$. The building gives a fiber sequence

$$
Q_{U}\left(K_{0}^{p, \ell}\right) \rightarrow \prod_{\mathfrak{P} \mid u} K\left(E_{\mathfrak{P}}\right)^{h \mathcal{O}_{F}^{\times}} \stackrel{\prod \psi^{q}-1}{\longrightarrow} \prod_{\mathfrak{P} \mid u} K\left(E_{\mathfrak{P}}\right)^{h \mathcal{O}_{F}^{\times}}
$$





\section{Bibliography}

[Ada66] J. F. Adams, On the groups $J(X) . I V$, Topology 5 (1966), 21-71.

[Ada74] Stable homotopy and generalised homology, University of Chicago Press, Chicago, Ill., 1974, Chicago Lectures in Mathematics.

[AN02] Peter Abramenko and Gabriele Nebe, Lattice chain models for affine buildings of classical type, Math. Ann. 322 (2002), no. 3, 537-562.

[Bak90] Andrew Baker, Hecke operators as operations in elliptic cohomology, J. Pure Appl. Algebra 63 (1990), no. 1, 1-11.

[Bak99] Hecke operations and the Adams $E_{2}$-term based on elliptic cohomology, Canad. Math. Bull. 42 (1999), no. 2, 129-138.

[Beh] Mark Behrens, Buildings, elliptic curves, and the K(2)-local sphere, To appear in Amer. J. Math.

[Beh06] A modular description of the $K(2)$-local sphere at the prime 3 , Topology 45 (2006), no. 2, 343-402.

[BL06] Mark Behrens and Tyler Lawson, Isogenies of elliptic curves and the Morava stabilizer group, J. Pure Appl. Algebra 207 (2006), no. 1, 37-49.

[Bla01] Benjamin A. Blander, Local projective model structures on simplicial presheaves, $K$ Theory 24 (2001), no. 3, 283-301.

[Bor66] Armand Borel, Introduction to automorphic forms, Algebraic Groups and Discontinuous Subgroups (Proc. Sympos. Pure Math., Boulder, Colo., 1965), Amer. Math. Soc., Providence, R.I., 1966, pp. 199-210.

[Bou79] A. K. Bousfield, The localization of spectra with respect to homology, Topology 18 (1979), no. 4, 257-281.

[Clo91] Laurent Clozel, Représentations galoisiennes associées aux représentations automorphes autoduales de GL(n), Inst. Hautes Études Sci. Publ. Math. (1991), no. 73, 97-145.

[Dav06] Daniel G. Davis, Homotopy fixed points for $L_{K(n)}\left(E_{n} \wedge X\right)$ using the continuous action, J. Pure Appl. Algebra 206 (2006), no. 3, 322-354.

[Dem72] Michel Demazure, Lectures on p-divisible groups, Springer-Verlag, Berlin, 1972, Lecture Notes in Mathematics, Vol. 302.

[DH95] Ethan S. Devinatz and Michael J. Hopkins, The action of the Morava stabilizer group on the Lubin-Tate moduli space of lifts, Amer. J. Math. 117 (1995), no. 3, 669-710.

[DH04] - Homotopy fixed point spectra for closed subgroups of the Morava stabilizer groups, Topology 43 (2004), no. 1, 1-47.

[DHI04] Daniel Dugger, Sharon Hollander, and Daniel C. Isaksen, Hypercovers and simplicial presheaves, Math. Proc. Cambridge Philos. Soc. 136 (2004), no. 1, 9-51.

[Dri74] V. G. Drinfel'd, Elliptic modules, Mat. Sb. (N.S.) 94(136) (1974), 594-627, 656.

[Eis95] David Eisenbud, Commutative algebra, Graduate Texts in Mathematics, vol. 150, Springer-Verlag, New York, 1995, With a view toward algebraic geometry.

[Fis75] Robert Fisher, Endomorphisms of abelian schemes, Ph.D. thesis, Harvard University, 1975.

[Gar97] Paul Garrett, Buildings and classical groups, Chapman \& Hall, London, 1997.

[GH04] P. G. Goerss and M. J. Hopkins, Moduli spaces of commutative ring spectra, Structured ring spectra, London Math. Soc. Lecture Note Ser., vol. 315, Cambridge Univ. Press, Cambridge, 2004, pp. 151-200.

[GHMR05] Paul Goerss, Hans-Werner Henn, Mark Mahowald, and Charles Rezk, A resolution of the $K(2)$-local sphere at the prime 3, Ann. of Math. (2) 162 (2005), no. 2, 777-822.

[GJ98] P. G. Goerss and J. F. Jardine, Localization theories for simplicial presheaves, Canad. J. Math. 50 (1998), no. 5, 1048-1089. 
[GM95] J. P. C. Greenlees and J. P. May, Completions in algebra and topology, Handbook of algebraic topology, North-Holland, Amsterdam, 1995, pp. 255-276.

[Goe95] Paul G. Goerss, Homotopy fixed points for Galois groups, The Čech centennial (Boston, MA, 1993), Contemp. Math., vol. 181, Amer. Math. Soc., Providence, RI, 1995, pp. 187-224.

[Gro67] A. Grothendieck, Éléments de géométrie algébrique. IV. Étude locale des schémas et des morphismes de schémas IV, Inst. Hautes Études Sci. Publ. Math. (1967), no. 32, 361.

[Har77] Robin Hartshorne, Algebraic geometry, Springer-Verlag, New York, 1977, Graduate Texts in Mathematics, No. 52.

[Hel78] Sigurdur Helgason, Differential geometry, Lie groups, and symmetric spaces, Pure and Applied Mathematics, vol. 80, Academic Press Inc. [Harcourt Brace Jovanovich Publishers], New York, 1978.

[Hen07] Hans-Werner Henn, On finite resolutions of $K(n)$-local spheres, Elliptic cohomology, London Math. Soc. Lecture Note Ser., vol. 342, Cambridge Univ. Press, Cambridge, 2007, pp. 122-169.

[Hid04] Haruzo Hida, p-adic automorphic forms on Shimura varieties, Springer Monographs in Mathematics, Springer-Verlag, New York, 2004.

[Hon68] Taira Honda, Isogeny classes of abelian varieties over finite fields, J. Math. Soc. Japan 20 (1968), 83-95.

[HS98] Michael J. Hopkins and Jeffrey H. Smith, Nilpotence and stable homotopy theory. II, Ann. of Math. (2) 148 (1998), no. 1, 1-49.

[HS99] Mark Hovey and Neil P. Strickland, Morava K-theories and localisation, Mem. Amer. Math. Soc. 139 (1999), no. 666, viii+100.

[HT01] Michael Harris and Richard Taylor, The geometry and cohomology of some simple Shimura varieties, Annals of Mathematics Studies, vol. 151, Princeton University Press, Princeton, NJ, 2001, With an appendix by Vladimir G. Berkovich.

[Jar87] J. F. Jardine, Simplicial presheaves, J. Pure Appl. Algebra 47 (1987), no. 1, 35-87.

[Jar97] Generalized étale cohomology theories, Progress in Mathematics, vol. 146, Birkhäuser Verlag, Basel, 1997.

[Jar00] - Presheaves of symmetric spectra, J. Pure Appl. Algebra 150 (2000), no. 2, $137-154$.

[Kat73] Nicholas M. Katz, p-adic properties of modular schemes and modular forms, Modular functions of one variable, III (Proc. Internat. Summer School, Univ. Antwerp, Antwerp, 1972), Springer, Berlin, 1973, pp. 69-190. Lecture Notes in Mathematics, Vol. 350.

[Kat81] N. Katz, Serre-Tate local moduli, Algebraic surfaces (Orsay, 1976-78), Lecture Notes in Math., vol. 868, Springer, Berlin, 1981, pp. 138-202.

[Kot92] Robert E. Kottwitz, Points on some Shimura varieties over finite fields, J. Amer. Math. Soc. 5 (1992), no. 2, 373-444.

[Lan76] Peter S. Landweber, Homological properties of comodules over $M \mathrm{U}_{*}(M \mathrm{U})$ and $B P_{*}(B P)$, Amer. J. Math. 98 (1976), no. 3, 591-610.

[Lau99] Gerd Laures, The topological q-expansion principle, Topology 38 (1999), no. 2, 387425.

[LT66] Jonathan Lubin and John Tate, Formal moduli for one-parameter formal Lie groups, Bull. Soc. Math. France 94 (1966), 49-59.

[Lur] Jacob Lurie, A survey of elliptic cohomology, preprint.

[Mah70] Mark Mahowald, The order of the image of the J-homomorphisms, Bull. Amer. Math. Soc. 76 (1970), 1310-1313.

[Mil79] J. S. Milne, Points on Shimura varieties mod $p$, Automorphic forms, representations and $L$-functions (Proc. Sympos. Pure Math., Oregon State Univ., Corvallis, Ore., 1977), Part 2, Proc. Sympos. Pure Math., XXXIII, Amer. Math. Soc., Providence, R.I., 1979, pp. 165-184.

[Mil98] _ Abelian varieties, 1998

[Mil05] Algebraic groups and arithmetic groups, 2005.

[Min99] Norihiko Minami, Hecke algebras and cohomotopical Mackey functors, Trans. Amer. Math. Soc. 351 (1999), no. 11, 4481-4513.

[Mor89] Jack Morava, Forms of K-theory, Math. Z. 201 (1989), no. 3, 401-428. 
[MR] Mark Mahowald and Charles Rezk, On topological modular forms of level 3, Preprint.

[MRW77] Haynes R. Miller, Douglas C. Ravenel, and W. Stephen Wilson, Periodic phenomena in the Adams-Novikov spectral sequence, Ann. Math. (2) 106 (1977), no. 3, 469-516.

[MS74] John W. Milnor and James D. Stasheff, Characteristic classes, Princeton University Press, Princeton, N. J., 1974, Annals of Mathematics Studies, No. 76.

[Mum65] David Mumford, Geometric invariant theory, Ergebnisse der Mathematik und ihrer Grenzgebiete, Neue Folge, Band 34, Springer-Verlag, Berlin, 1965. MR MR0214602 (35 \#5451)

[Mum70] _ Abelian varieties, Tata Institute of Fundamental Research Studies in Mathematics, No. 5, Published for the Tata Institute of Fundamental Research, Bombay, 1970.

[Nau] Niko Naumann, Arithmetically defined dense subgroups of Morava stabilizer groups, To appear in Compositio Math.

[Nau07] N. Naumann, The stack of formal groups in stable homotopy theory, Advances in Math. 215 (2007), no. 2, 569-600.

[PR94] Vladimir Platonov and Andrei Rapinchuk, Algebraic groups and number theory, Pure and Applied Mathematics, vol. 139, Academic Press Inc., Boston, MA, 1994, Translated from the 1991 Russian original by Rachel Rowen.

[Rav84] Douglas C. Ravenel, Localization with respect to certain periodic homology theories, Amer. J. Math. 106 (1984), no. 2, 351-414.

[Rav86] Complex cobordism and stable homotopy groups of spheres, Pure and Applied Mathematics, vol. 121, Academic Press Inc., Orlando, FL, 1986.

[Rav92] Nilpotence and periodicity in stable homotopy theory, Annals of Mathematics Studies, vol. 128, Princeton University Press, Princeton, NJ, 1992, Appendix C by Jeff Smith.

[Rez98] Charles Rezk, Notes on the Hopkins-Miller theorem, Homotopy theory via algebraic geometry and group representations (Evanston, IL, 1997), Contemp. Math., vol. 220, Amer. Math. Soc., Providence, RI, 1998, pp. 313-366.

[Sad92] Hal Sadofsky, The root invariant and $v_{1}$-periodic families, Topology 31 (1992), no. 1, $65-111$.

[Sch85] Winfried Scharlau, Quadratic and Hermitian forms, Grundlehren der Mathematischen Wissenschaften [Fundamental Principles of Mathematical Sciences], vol. 270, SpringerVerlag, Berlin, 1985.

[Shi81] Katsumi Shimomura, Novikov's $\operatorname{Ext}^{2}$ at the prime 2, Hiroshima Math. J. 11 (1981), no. 3, 499-513.

[Str99] Neil P. Strickland, Formal schemes and formal groups, Homotopy invariant algebraic structures (Baltimore, MD, 1998), Contemp. Math., vol. 239, Amer. Math. Soc., Providence, RI, 1999, pp. 263-352.

[SW02] Katsumi Shimomura and Xiangjun Wang, The homotopy groups $\pi_{*}\left(L_{2} S^{0}\right)$ at the prime 3, Topology 41 (2002), no. 6, 1183-1198.

[SY95] Katsumi Shimomura and Atsuko Yabe, The homotopy groups $\pi_{*}\left(L_{2} S^{0}\right)$, Topology 34 (1995), no. 2, 261-289.

[Tat67] J. T. Tate, $p$-divisible groups., Proc. Conf. Local Fields (Driebergen, 1966), Springer, Berlin, 1967, pp. 158-183.

[Tat71] John Tate, Classes d'isogény des variétiés abéliennes sur un corps fini (d'après t. honda), Séminaire Bourbaki, Exp. 352, Lecture Notes in Math., vol. 179, SpringerVerlag, Berlin, 1971.

[WM71] W. C. Waterhouse and J. S. Milne, Abelian varieties over finite fields, 1969 Number Theory Institute (Proc. Sympos. Pure Math., Vol. XX, State Univ. New York, Stony Brook, N.Y., 1969), Amer. Math. Soc., Providence, R.I., 1971, pp. 53-64.

[Yos83] Tomoyuki Yoshida, On G-functors. II. Hecke operators and G-functors, J. Math. Soc. Japan 35 (1983), no. 1, 179-190. 



\section{Index}

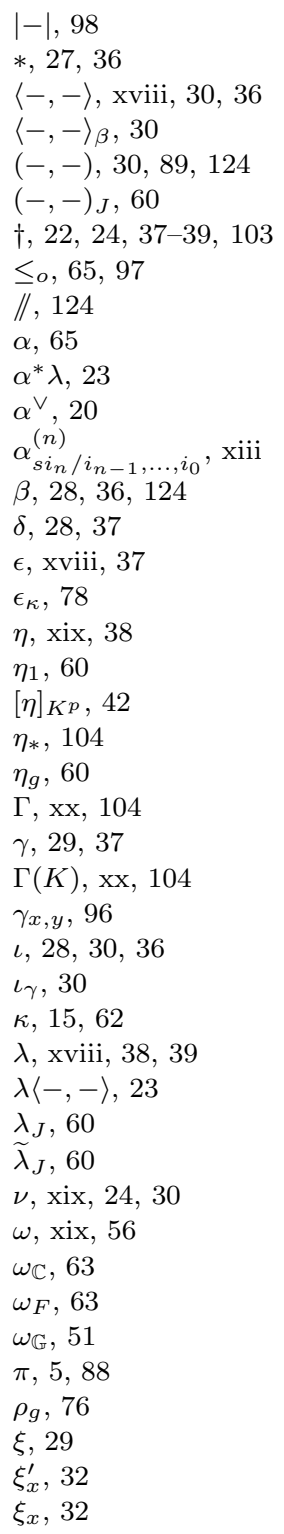

$A$, xvii, 38,39

$A^{\vee}, 19$

$\mathbb{A}$, xix

A, 103

$\widehat{A}$, xvii

$A_{J}, 59$

$\mathbb{A}^{S}$, xix

$\mathbb{A}_{S}$, xix

$\mathrm{AbVar}_{k}^{0}, 5$

$A\left[\ell^{\infty}\right], 13$

$A(p), 2$

$A\left[p^{\infty}\right], 1$

$A\left[p^{i}\right], 2$

$A\left[\right.$ tor $\left.^{p}\right], 14$

$A(u), 4$

$\widehat{A}_{u}$, xviii

$B$, xviii, $9,27,36$

$\mathcal{B}, 96$

B., 96

$\mathcal{B}_{\bullet}^{\prime}, 96$

$\mathcal{B}(G L), 88$

$\mathcal{B}(G U), 93$

$\mathcal{B}(S L), 88$

$\mathcal{B}(U)$, xxi, 90

$C, 27,36$

$c, 5,27$

$C_{\infty}, 60$

$\mathrm{Cl}(F), 123$

CoInd, 71

Cor $(S, T), 75$

D, 103

$d, 100$

$\operatorname{Def}_{\overline{\mathbb{G}}}, 45$

disc, 32

E, 124

$\mathcal{E}$, xix

$E_{\overline{\mathbb{G}}}, 52$

$\mathcal{E}_{\mathbb{G}}, 52$

$E\left(\Gamma\left(K^{p, S}\right)\right), \mathrm{xxi}$

$\mathcal{E}\left(K^{p}\right), 56$

$e_{i}, 37$

$E_{n}, \mathrm{x}$

End $^{0}(A), 5$ 
INDEX

$F$, xvii, 9, 27, 36, 81, 122

Fr, 114

$G, 65,80,96,118$

$\mathbb{G}^{\vee}, 2$

$\overline{\mathbb{G}}, 45$

$\mathbb{G}^{0}, 3$

$G^{1}, 118$

$\mathbb{G}_{E}, 2,51$

$G L_{C}, 30$

$\mathbb{G}(T), 45$

$G U$, xix, 30, 36

$G U^{1}, \mathrm{xx}, 91,95$

$G U_{\mathbf{A}}, 103$

$G U(\mathbb{R})^{+}, 61$

$G U_{V_{\ell}(A)}, 25$

$G U\left(\mathbb{Z}_{(p)}\right)^{+}, 62$

Gal, x, 114

$\mathbb{G}^{e t}, 3$

$G_{\ell}, 96$

$\mathbb{G}_{n}, \mathrm{x}, 121$

$H, 96$

$\mathcal{H}, 61,81$

$H_{(A, i, \lambda)}, 24$

$H_{n}, \mathrm{x}$

$\operatorname{Hom}^{0}\left(A, A^{\prime}\right), 5$

$\|I\|$, xii

$i$, xviii, 38, 39, 60

$i_{K^{\prime}}^{K}, 76$

$i_{\eta}, 104$

$i_{n, Z}, 68$

Ind, 72

$\operatorname{inv}_{x}, 6$

$J, \mathrm{xi}, 59$

$j, 59$

$K, 88,89,125$

$\underline{k}, 63$

$K\left(E_{\mathfrak{P}}\right), 125$

$K_{\alpha}, 122$

$\operatorname{Ker}(\phi), 14$

$K_{\infty}, 62$

$K_{\ell}(\sigma)$, xxii, 100

$K^{p}$, xix, 41

$K_{0}^{p}$, xix, $15,36,124$

$K_{+}^{p, S}, \mathrm{xx}, 95$

$K_{1,+}^{p, S}, \mathrm{xx}, 95$

$K^{p, \ell}, 95$

$K(\sigma)$, xxii, 100

$L$, xix, 36, 67, 70

$L^{\#}, 90$

$L^{\prime}, 73$

$L^{*}, 60$

$L_{1}, 20$

$\mathcal{L}^{2}, 67,70,73$

$\mathcal{L}_{K_{0}^{p}}(A), 15$

Lie $A, 57$

Lie $A^{+}, 59$

Lie $A^{-}, 59$
Lie $\mathbb{G}, 57$

$L^{p}, 15,36$

$M, 5$

$\mathcal{M}, 80$

$M^{+}, 5$

$\mathcal{M}_{F G}, 53$

$M(I), 114$

$M(I)^{0}$, ix

$M_{n} X$, ix

$M U P, 53$

$\operatorname{Map}_{\Gamma}(-,-)^{s m}, 118$

$\operatorname{Map}_{U}(-,-), 97$

$\operatorname{Map}_{U}(-,-), 97$

$\overline{\operatorname{Map}}(-,-)^{s m}, 97$

$\operatorname{Map}_{G}(-,-)^{s m}, 71$

$\operatorname{Map}(-,-)^{s m}, 97$

$\overline{\mathcal{M}_{G}^{c o}}, 75$

$M_{t}\left(\Gamma_{0}(N)\right)_{R}$, xiv

${ }_{H} N_{H^{\prime}}, 66$

$\mathcal{O}, 88$

$\mathcal{O}_{B}$, xviii, 36

$\mathcal{O}_{C}, 36$

$\mathcal{O}_{D}, 103$

$\mathcal{O}_{F}$, xvii, 36,122

$\mathrm{Orb}^{c o}, 80$

$P G U, 37$

$\left[p^{i}\right], 1$

$\mathrm{Pic}_{A / k}^{0}, 19$

$\mathrm{Pic}_{A / k}, 19$

PreSp $\left(\operatorname{Set}_{G}^{s m, \alpha}\right), 70$

$Q, 96$

$Q^{\bullet}$, xxii, 100

$Q_{G U}, \mathrm{xx}, 95$

$Q_{U}, \mathrm{xx}, 95$

$Q(\ell)$, xii

$R, 67,70$

$R^{\prime}, 73$

$r_{K^{\prime}}^{K}, 76$

Res, 71, 118

$S(-), 98$

$s$ Pre $\left(\operatorname{Set}_{G}^{s m, \alpha}\right), 67$

$s \operatorname{Set}_{G}^{s m}, 67$

$s \operatorname{Shv}\left(\operatorname{Set}_{G}^{s m, \alpha}\right), 67$

$\operatorname{Set}_{G}^{c o, \alpha}, 72$

Set $_{G}^{s m}, 65$

$\operatorname{Set}_{G}^{\text {sm, }}, 65$

$S h$, xviii

$\operatorname{Sh}\left(K^{p}\right)$, xix, 42

$S h\left(K^{p}\right)_{\mathbb{C}}, 57,59$

$S h\left(K^{p}\right)_{F}, 57,59$

$\operatorname{Sh}\left(K^{p}\right)_{\mathbb{F}_{q}}, 114$

$S h\left(K^{p}\right)_{F_{u}}, 57$

$S h\left(K^{p}\right)^{[n]}, \mathrm{xx}, 103$

$\operatorname{Sh}\left(K^{p}\right)_{(u)}, 57,58$

$\operatorname{Shv~} \operatorname{Sp}\left(\operatorname{Set}_{G}^{s m, \alpha}\right), 70$

$\mathbb{S}_{n}, \mathrm{x}, 104$

$\mathrm{Sp}_{\Gamma}, 118$ 


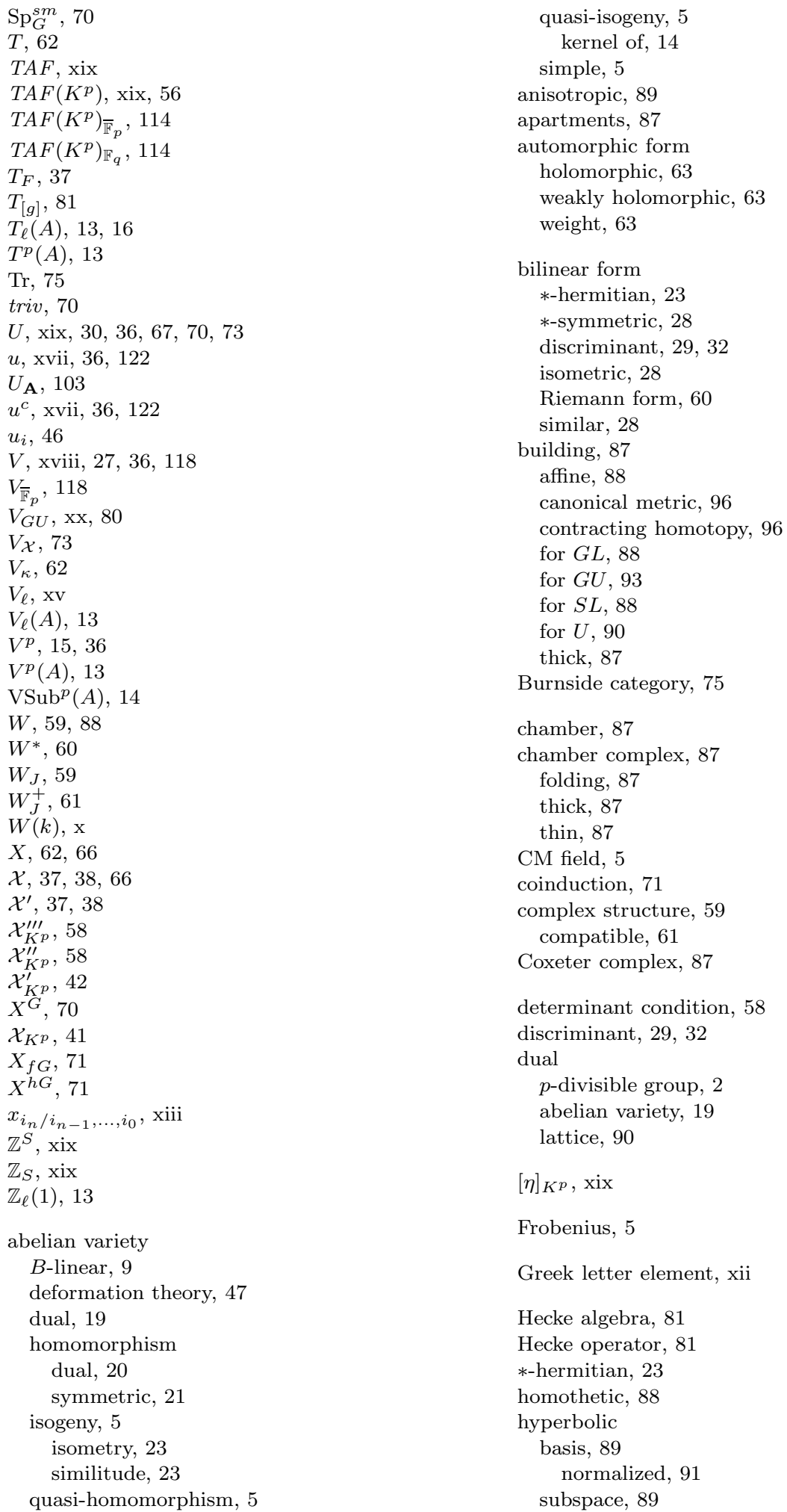

quasi-isogeny, 5

kernel of, 14

simple, 5

anisotropic, 89

apartments, 87

automorphic form

holomorphic, 63

weakly holomorphic, 63

weight, 63

bilinear form

*-hermitian, 23

*-symmetric, 28

discriminant, 29, 32

isometric, 28

Riemann form, 60

similar, 28

building, 87

affine, 88

canonical metric, 96

contracting homotopy, 96

for $G L, 88$

for $G U, 93$

for $S L, 88$

for $U, 90$

thick, 87

Burnside category, 75

chamber, 87

chamber complex, 87

folding, 87

thick, 87

thin, 87

CM field, 5

coinduction, 71

complex structure, 59

compatible, 61

Coxeter complex, 87

determinant condition, 58

discriminant, 29, 32

dual

p-divisible group, 2

abelian variety, 19

lattice, 90

$[\eta]_{K^{p}}$, xix

Frobenius, 5

Greek letter element, xii

Hecke algebra, 81

Hecke operator, 81

*-hermitian, 23

homothetic, 88

hyperbolic

basis, 89

normalized, 91

subspace, 89 


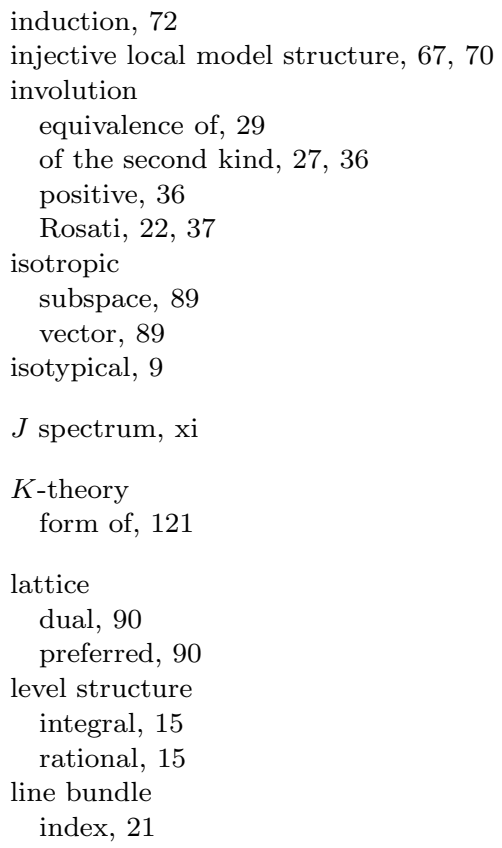

Mackey functor, 76

monochromatic layer, ix

Newton polygon, 3

$p$-adic type, 8

minimal, 9

over $F, 9$

$p$-divisible group, 1

Cartier dual, 2

deformation theory, 45

dimension, 3

fppf sheaf, 45

height, 1

homomorphism, 2

isogeny, 2

of an abelian scheme, 2

simple, 3

panel, 87

Poincaré bundle, 20

polarization, 21

$R$-polarization, 37

compatible, 23

equivalence of, 22

induced, 23

of an abelian scheme, 37

similitude class, 24

weak, 22

preferred lattice, 90

projective local model structure, 68

restriction, 71

Riemann form, 60

Rosati involution, 22, 37 semi-simplicial set, 87

Shimura stack, xviii, 35, 42

signature, 32

similitude, 23

similitude class, 24

similitude group, 30

similitude norm, 30

simplicial complex, 87

slope, 3

smooth

$G$-set, 65

simplicial, 67

$G$-spectrum, xx, 69

homotopy fixed points, $\mathrm{xx}, 71$

Tate module, 13

topological automorphic forms, xix, 56

totally isotropic, 89

transfer, 75

uniformization

integral, 15

rational, 15

unitary group, 30

virtual subgroup, 14

weakly even periodic, 51

Weil $q$-integer, 6

Weil pairing, 22

Witt index, 89 